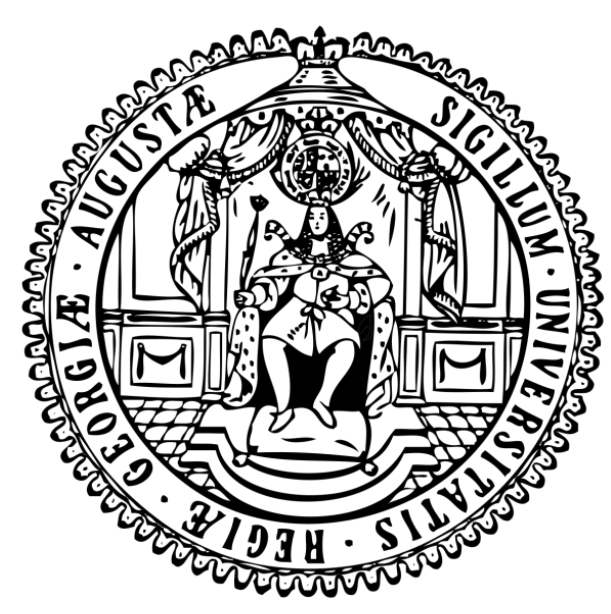

\title{
Neutrophil Extracellular Trap (NET) Formation: From Fundamental Biophysics to Delivery of Nanosensors
}

\author{
Dissertation \\ for the award of the degree \\ "Doctor rerum naturalium" \\ of the Georg-August-Universität Göttingen
}

within the doctoral program

Physics of Biological and Complex Systems of the Georg-August University School of Science (GAUSS)

\author{
submitted by \\ Daniel Meyer
}

from Hildesheim, Germany

Göttingen 2019 
Thesis Committee \& Members of the Examination Board

Dr. Sebastian Kruss ( $1^{\text {st }}$ Referee and Supervisor)

Institute of Physical Chemistry | Georg-August University Göttingen

\section{Prof. Dr. Jörg Enderlein (2 ${ }^{\text {nd }}$ Referee)}

$3^{\text {rd }}$ Physical Institute, Biophysics \& Complex Systems | Georg-August University Göttingen

\section{Dr. Thomas Burg}

Max-Planck Institute for Biophysical Chemistry, Dep. Micro- and Nanotechnology / Göttingen

\section{Further members of the Examination Board}

Dr. Karen Alim

Max-Planck Institute for Dynamics and Self-Organization / Göttingen

\section{Prof. Claudia Steinem}

Institute of Organic and Biomolecular Chemistry | Georg-August University Göttingen

\section{Prof. Stefan Jakobs}

Max-Planck Institute for Biophysical Chemistry, Dep. NanoBiophotonic / Göttingen

\section{Date of oral examination:}

26.6.2019 
Not all heroes wear capes.

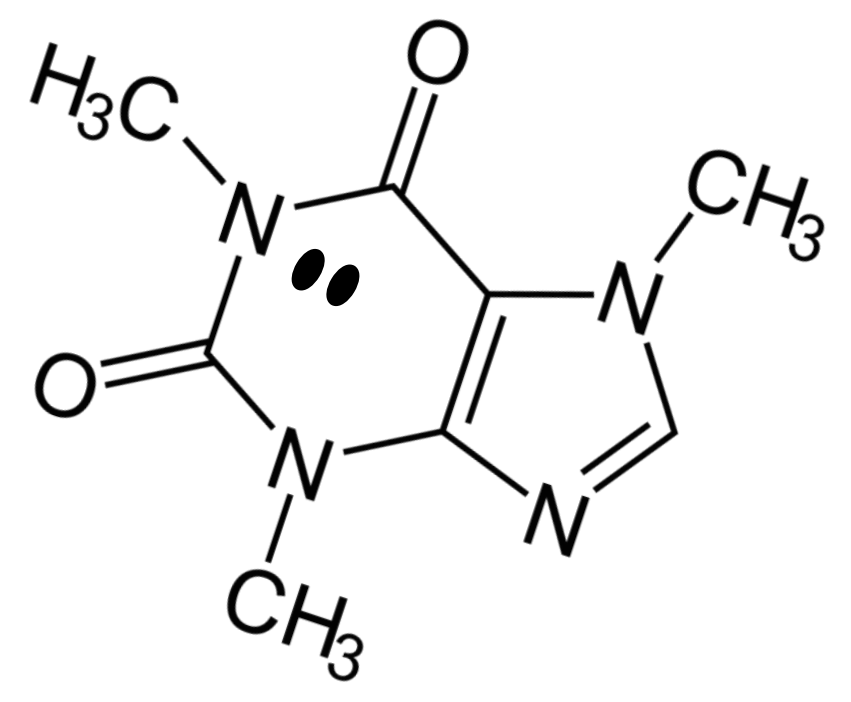

Caffeine 


\section{Abstract}

Immune cells have remarkable properties. They are able to migrate long distances, cross dense cell barriers and use a wide range of tools to identify and fight foreign materials in our body. In this context, neutrophil extracellular trap formation (NETosis), as their latest tool and a new type of cell death, has received much attention in the last years. During NETosis, leukocytes, such as neutrophilic granulocytes (neutrophils), undergo massive morphological changes leading to intracellular chromatin decondensation, membrane disruption and a final release of their nuclear content in the form of neutrophil extracellular traps (NETs). Even though many studies tried to elucidate these unique alterations, the general course of NETosis is still poorly understood.

For this reason, this cumulative thesis investigated NETosis for the first time from a biophysical point of view, primarily to understand how the cell rearranges its interior and to identify physical driving forces behind the process. Furthermore, the insights of this research were utilized to transform neutrophils into carrier systems capable of uptaking, transporting and the releasing fluorescent nanosensors and a theoretical study was conducted that examined the best kinetic requirements for molecular imaging with these functional materials.

In the first part of this thesis, the entropic pressure generated by the swelling chromatin network was identified as a driving force of NETosis. Using optical and mechanical approaches, the NETotic process could be classified into three distinct phases, including a first enzymatic/signaling driven state and a point of no return. Atomic force microscopy measurements further showed cytoskeletal degradation, which decreased both the cells Young's modulus and membrane tension, while also revealing chromatin swelling forces that are capable of disrupting the weakened cell membrane. Therefore, this work was able to identify NETosis as a non-equilibrium process that is orchestrated by balancing forces of intracellular components.

The second part further utilizes this perception of NETosis and transformed neutrophils into vehicles for material transport. Here, fluorescent single-walled carbon nanotube nanosensors (SWCNTs), known for their ability to detect biological compounds, served as cargo. Remarkably, live-cell imaging experiments showed that stimulated neutrophils were still able to migrate and react to chemical/mechanical cues before releasing their intracellular content. Likewise, transported and released sensors were fully functional and detected small molecules (neurotransmitters, reactive oxygen species). Therefore, NETosis-based delivery could become a powerful approach in biomedical applications.

Finally, the last part of this thesis evaluated the kinetics that nanosensors require to detect dynamic, chemical processes within biological systems. For this purpose, a theoretical framework based on kinetic Monte-Carlo simulations was generated and provided essential insights into the interplay of sensor/analyte interactions as well as their consequences on the optical output within molecular imaging processes.

Keywords: Active matter, chromatin, immune cells, NETosis, neutrophilic granulocytes, carbon nanotubes, sensors, cargo delivery, Monte-Carlo simulations 


\section{Zusammenfassung}

Immunzellen besitzen beindruckende Fähigkeiten: Angetrieben durch mechanisch/chemische Reize sind sie in der Lage in unserem Körper größte Entfernungen zurückzulegen, dichteste Zellbarrieren zu durchbrechen und Pathogene und fremdes Material zu erkennen und zu zerstören. Die Generierung von extrazellulären Netzen (NETose), einer erst kürzlich entdeckten Art des Zelltodes, zählt hierbei wohl zu ihren interessantesten, anti-inflammatorischen Strategien. Während der NETose durchlaufen bestimmte Leukozyten, wie etwa Neutrophile Granulozyten (Neutrophile), enorme morphologische Veränderungen: Initiiert durch die Dekondensation ihres eigenen Chromatins, vermengen sich ihre nuklearen und zytosolischen Inhalte, formen ein dichtes Netzwerk aus DNA und proteolytischen Enzymen und treten durch eine finale Ruptur der Zellmembran als großflächige, fangnetzartiges Gebilde hervor - den sogenannten neutrophilen extrazellulären Fallen (Neutrophil Extracellular Traps, NETs). Obgleich hierbei die Entstehung von NETs eine zentrale Rolle in der derzeitigen, immunologischen Forschung einnimmt, ist ihr zugrundeliegender Mechanismus bisher leider kaum verstanden.

Aus diesem Grund widmet sich diese kumulative Dissertation erstmals einer neuen, biophysikalischen Betrachtung von NETose und erschließt die treibende Kräfte hinter diesem neuartigem, anti-inflammatorischen Prozess. Zudem werden die gewonnenen Erkenntnisse genutzt, um aktivierte Neutrophile zu Transportsystemen für auf Nanoröhrenbasierende Nanosensoren umzufunktionieren und eine theoretische Arbeit vorgestellt, die die kinetischen Bedingungen ebensolcher Sensoren zugunsten chemischer Bildgebungsverfahren untersucht.

Dabei ließ sich im ersten Teil dieser Arbeit das Anschwellen des Chromatinnetzwerkes und des so entstehenden entropischen Drucks als zentrale Triebfeder der NETose identifizieren. Durch verschiedenste mikroskopische Konzepte unterstützt, konnte der NETotische Entstehungsablauf zunächst in drei charakteristische Phasen unterteilt und auf zellmechanische Veränderungen zurückgeführt werden. Hierbei zeugten vor allem Rasterkraftmikroskopieaufnahmen von einer Abnahme zellulärer Steifigkeit, sowie der Entstehung von intrazellulären Schwelldrücken, sodass die finale Freigabe der NETs als Folge destabilisierender Kräfte beschrieben werden konnte.

Im zweiten Teil wurden nun die mehrphasigen Eigenschaften aktivierter Neutrophiler mit den optischen Fähigkeiten einwandiger Kohlenstoff-Nanoröhrensensoren verbunden, um eine neue Art von zellbasiertem Nanomaterial-Transportsystem zu entwickeln. Wie sich hierbei durch Lebendzellaufnahmen zeigte, ließen sich gezielt stimulierte Neutrophile zu programmierbaren Beförderungsplattformen instrumentalisieren und ermöglichten eine gerichtete Überführung und Freigabe funktioneller Nanosensoren.

Im abschließenden Teil dieser Dissertation wurden zudem die kinetischen Bedingungen solcher Nanosensoren für chemische Bildgebungsverfahren theoretisch evaluiert. Zu diesem Zweck konnte eine Monte-Carlo-Simulation entwickelt werden, welche die dynamischen Interaktionen zwischen Sensor und Analyt reproduzieren und somit die räumlichdynamische Antwort von fluoreszenten Nanosensoren nachbilden konnte.

Schlüsselwörter: Aktive Materie, Chromatin, Immunzellen, NETose, Neutrophile Granulozyten, Karbon-Nanoröhren, Sensoren, Transportsysteme, Monte-Carlo Simulation 


\section{Table of Contents}

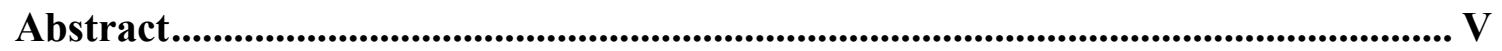

Zusammenfassung.......................................................................................................... II

Table of Contents ............................................................................................................... VII

List of Abbreviations .........................................................................................................

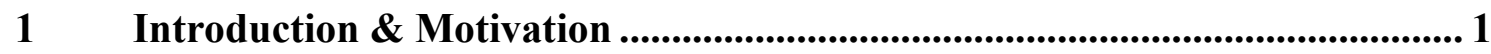

$2 \quad$ Scientific Background ......................................................................................... 3

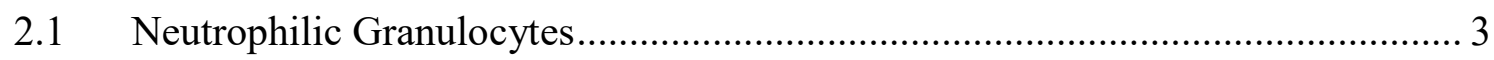

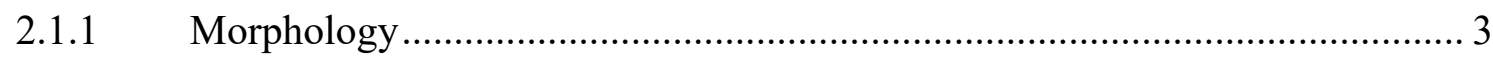

2.1.2 Neutrophil Development ......................................................................... 5

2.1.3 Migration and Mechanical Properties …………………………………....... 7

2.1.4 Immunological Response Functions …………………………………........ 10

2.2 Single-walled Carbon Nanotube Nanosensors ……………………………...... 15

2.2.1 Structural and Optical Properties of SWCNT ............................................. 15

2.2.2 Functionalization Methods \& Recognition Strategies of SWCNTs .............. 18

2.2.3 SWCNT based Chemical Imaging ........................................................... 20

2.3 Stochastic Chemical Kinetics \& Simulation Algorithms ……………………...... 22

$3 \quad$ Associated Publications............................................................................................ 27

3.1 Chromatin Swelling Drives Neutrophil Extracellular Trap Release ................... 27

3.2 Kinetic Requirements for Spatiotemporal Chemical Imaging with Fluorescent

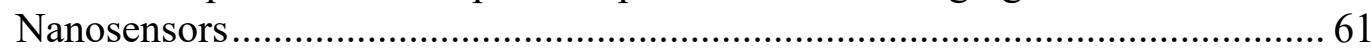

3.3 Programmed Transport and Release of Nanoscale Cargo by Immune Cells ...... 82

4 Publication Synopsis \& Contributions ............................................................... 109

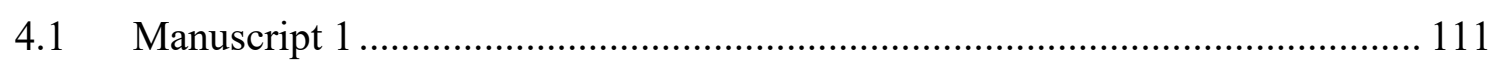

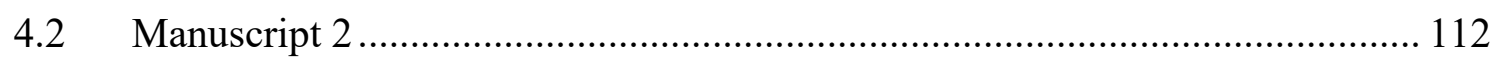

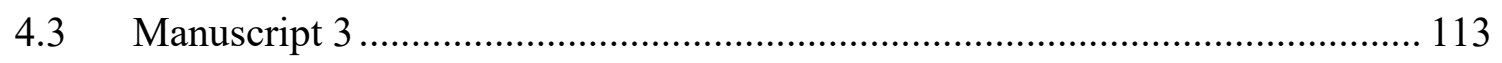

4.4 Cooperative publications ............................................................................ 114

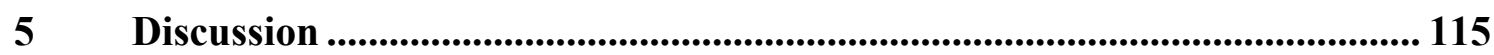

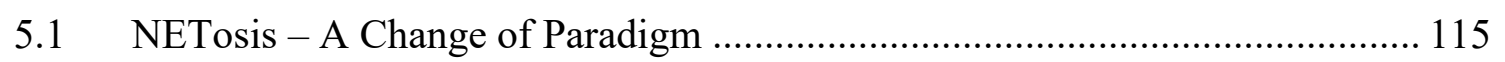

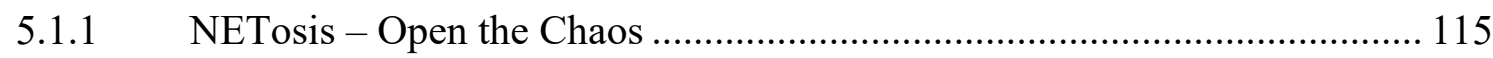

5.1.2 NETosis - A Force Speculation ............................................................ 118

5.2 A Theoretical Framework for Chemical Imaging Studies.................................. 120 
5.3 The Strength of Neutrophil-based Nanocarrier Systems..... 122

Acknowledgment / Danksagung ....................................................................................... i

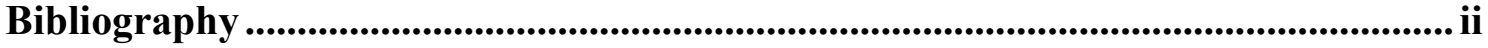

Curriculum vitae ............................................................................................................... xxii 


\section{List of Abbreviations}

\begin{tabular}{|c|c|}
\hline AFM & Atomic Force Microscope \\
\hline CXCL12 & CXC Motif Chemokine 12 \\
\hline CXCR4 & CXC Chemokine Receptor Type 4 \\
\hline DAMP & Damage-associated Molecular Pattern \\
\hline FcR & Fc-receptor \\
\hline FISH & Fluorescence In Situ Hybridization \\
\hline FMLP & N-Formylmethionyl-leucyl-phenylalanine \\
\hline FPR1 & Formyl Peptide Receptor 1 \\
\hline FRET & Förster Resonance Energy Transfer \\
\hline G-CSF & Granulocyte-Colony Stimulating Factor \\
\hline HUVEC & Human Umbilical Vein Endothelial Cells \\
\hline ICAM-1/2 & Intercellular Adhesion Molecules-1/2 \\
\hline IL-1alpha & Interleukin 1 Alpha \\
\hline IL-8 & Interleukin-8 \\
\hline JAM-A/C & Junctional Adhesion Molecule-A/C \\
\hline JNK & c-Jun N-terminal Kinase \\
\hline LINC complex & Linker of Nucleoskeleton and Cytoskeleton \\
\hline LPS & Lipopolysaccharide \\
\hline LTB4 & Lipid Attractant Leukotriene B4 \\
\hline MAC-1 & Macrophage-1 Antigen \\
\hline MPO & Myeloperoxidase \\
\hline NADPH & Reduced Nicotinamide Adenine Dinucleotide Phosphate \\
\hline $\mathrm{NE}$ & Neutrophil Elastase \\
\hline NET & Neutrophil Extracellular Trap \\
\hline NIR & Near-infrared \\
\hline NOX & NADPH Oxidase \\
\hline PAD4 & Peptidyl Arginine Deiminase 4 \\
\hline PAMP & Pathogen-associated Molecular Pattern \\
\hline PC12 cell & Pheochromocytoma Cell \\
\hline PECAM & Platelet Endothelial Cell Adhesion Molecules \\
\hline $\mathrm{PKC}$ & Protein Kinase C \\
\hline PMA & Phorbol-12-myristate-13-acetate \\
\hline PMN & Polymorphonuclear Cells \\
\hline PRR & Pattern Recognition Receptor \\
\hline PTK & Protein Tyrosine Kinase \\
\hline QY & Quantum Yield \\
\hline ROS & Reactive Oxygen Species \\
\hline SCK & Stochastic Chemical Kinetic \\
\hline SDF-1 & Stromal Derived Factor-1 \\
\hline SNARE & Soluble N-ethylmaleimide-sensitive-factor Attachment Receptor \\
\hline
\end{tabular}




$\begin{array}{ll}\text { SSA } & \text { Stochastic Simulation Algorithm } \\ \text { SWCNT } & \text { Single-Walled Carbon Nanotube } \\ \text { TLR } & \text { Toll-like Receptor } \\ \text { TNF- } \alpha & \text { Tumor Necrosis Factor Alpha } \\ \text { VCAM-1 } & \text { Vascular Cell Adhesion Protein 1 } \\ \text { VE-cadherin } & \text { Vascular Endothelial Cadherin } \\ \text { VLA4 } & \text { Very Late Antigen-4 }\end{array}$




\section{Introduction \& Motivation}

The human body is a fascinating and highly complex system. Almost every process, from immune responses to the beating of our heart, even the movement of our eyes while reading this text, is a result of a large amount of dynamic chemical and mechanical interactions, taking place on multiple length and time scales. For this reason, understanding this complexity from the single molecule to emergent long-term behavior is one of the greatest challenges in natural science.

On this account, our immune system can be seen as a crucial example highlighting many of those challenges. While inherent immune cells are able to use complex recognition concepts on a molecular scale and respond to chemical cues within milliseconds, their actions can lead to an array of severe consequences for the whole organism: reactions to acute inflammations, for example, result in tissue modifications and can last hours or even up to days, whereas other effects, such as immunity after vaccination or the development of cancer, still influences our body after decades. Thus, to tackle such a complex framework, the development of novel strategies combining the input of chemistry, physics and biology, becomes essential.

With this in mind, shedding some light into the complexity of immune cells became also a focal point of motivation for this work. Therefore, this cumulative thesis aims to investigate the unique properties of a specific type of leukocyte, the neutrophilic granulocytes (neutrophils), for the first time from a biophysical point of view and is complemented by innovative nanosensor approaches.

For that reason, this doctoral thesis is divided into three different manuscripts (chapter 3). Within the first study (chapter 3.1), neutrophils are investigated in respect to a novel immune defense strategy, the formation of neutrophil extracellular traps (NETs), and the underlying driving forces of this process are characterized using several optical and mechanical concepts. Furthermore, the second work (chapter 3.3) utilizes the consequently generated perception of NETotic cells and transform neutrophils into a programmable carrier system for single-walled carbon nanotube-based optical biosensors (SWCNTs). Lastly, the third study (chapter 3.2) also addresses the kinetical aspects of such sensors and introduces a theoretical framework for chemical imaging purposes.

Based on that, the proceeding pages are organized as follows: first, a theoretical background will be provided, followed by the presentation of the three manuscripts and an extensive summary of each work. Thereupon, the most essential findings of each study will be discussed and future perspectives will be given. 


\section{Scientific Background}

As mentioned in the preceding pages, the studies comprised in this thesis combined a wide range of methodologies and subject areas ranging from experimental, live-cell characterization of immune cells to theoretical simulations of nanotube-based nanosensors. All the more, it is vital to provide a certain level of knowledge at the beginning. For this reason, the following chapter is meant to familiarize oneself with the merits and restrictions of the used subject areas and shall provide a scientific basis for the upcoming presentation and discussion sections.

\subsection{Neutrophilic Granulocytes}

The term "neutrophilic granulocytes", also known as "neutrophils" or "neutrocytes", describes a specific kind of leukocyte and the primarily used cell system in this project compendium. With over $60 \%$ of all leukocytes in the bloodstream and even $80 \%$ during a bacterial infection, neutrophils constitute the most occurring type of white blood cell in mammalian life and act predominantly as the first line of defense against pathogens or other harmful intruders within our bodies. ${ }^{1,2}$ While already a century of dedicated research underlines their importance within the complex framework that is the immune system, their acknowledged anti-inflammatory toolkit has grown vast and comprises a large number of biochemical and mechanical response pathways ranging from single cell interactions up to permanent alterations of the adaptive immune memory. ${ }^{3-5}$ For that reason, this section aims to review the primary functions of neutrophilic granulocytes and will first focus on their general properties, such as their appearance or evolution, before their immunologic response functions and activities will be presented.

\subsubsection{Morphology}

Initially, the first usage of the term "neutrophil" can be backtracked to the very end of the $19^{\text {th }}$ century in which Paul Ehrlich, Nobel prize winner and at that time professor of the Charite in Berlin, could identify three different types of leukocytes in blood for the first time using customized coal tar dyes. ${ }^{6,7}$ As he proclaimed, these species of "polymorphous nuclei" containing cells could be distinguished by $\mathrm{pH}$-values of hundreds of small, cytoplasmic granules, which, for each cell type, appeared to be stainable with either acidic dyes (eosinophils), basic ones (basophils) or neutral dyes (neutrophils). 
Years later, all of these three species were correctly classified as a new leukocyte category, commonly known as the polymorphonuclear cell family (PMNs), as they did not only share the same developmental origin but also the most common phenotypic properties on which neutrophils can be recognized nowadays: their multilobular nucleus. ${ }^{2,8}$

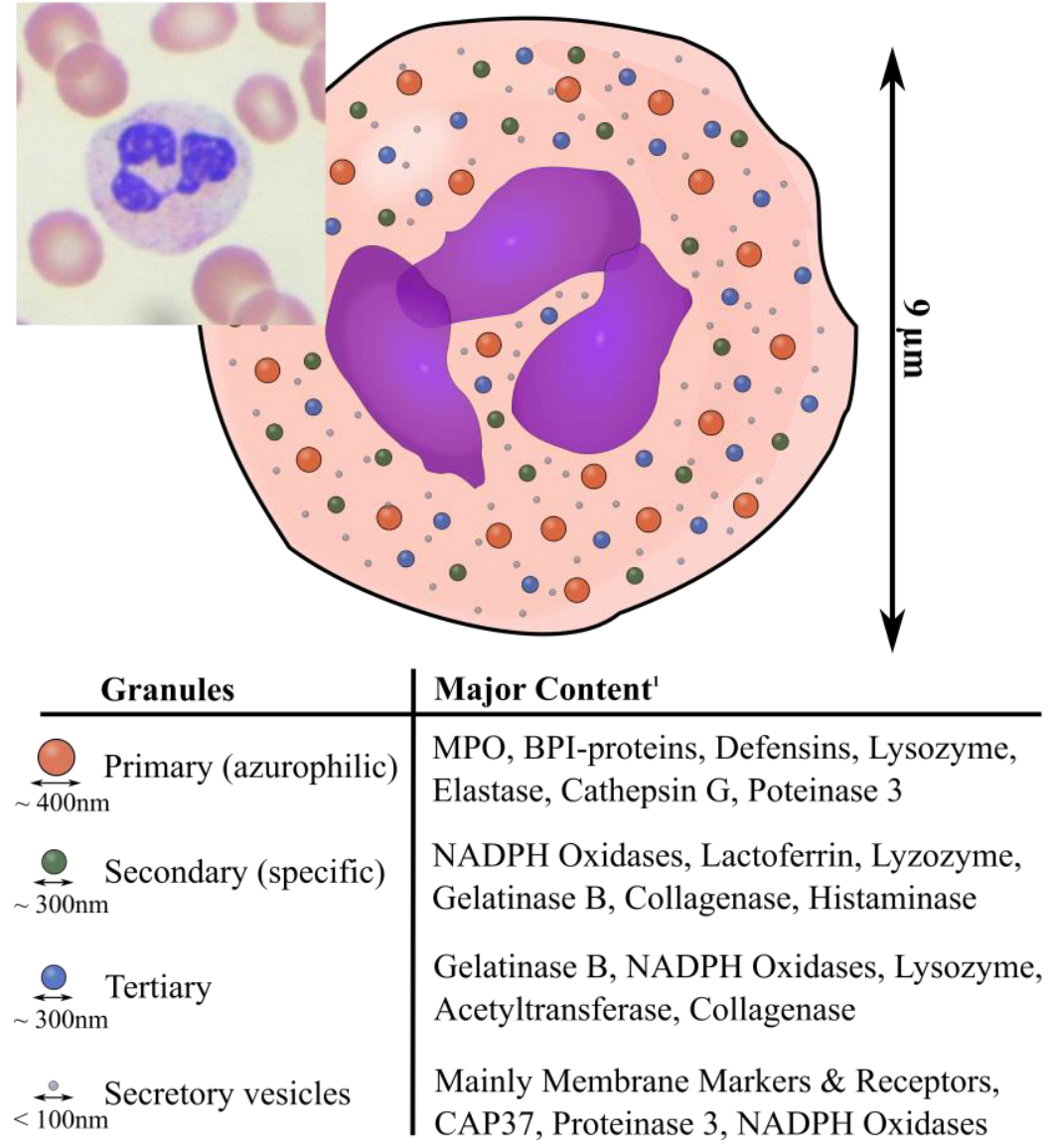

Figure 1: Morphology of neutrophilic granulocytes. As one of the members in the polymorphonuclear cell family (PMN), neutrophilic granulocytes are most often recognized by their 3-5 interconnected, multi-lobular nuclei as well as hundreds of carrier-granules within the intracellular fluid. Dissected by their size and functional content, the latter is comprised of 4 different types: primary (azurophilic) granules are home of most of the proteolytic enzymes and define the dominant and largest type of vesicles within the cell. In contrast, secondary (specific) granules are smaller but usually show receptor proteins or NADPH oxidases as an additional load. Tertiary (gelatinase) granules and scretory vesicles are finally either defined by their huge number of stored gelatinase particles or define the carrier system of a multiple of membrane and receptor markers. ${ }^{1}$ The above shown table contains only the main (antimicrobial) content of associated granules and intentionally leaves out content of minor concentrations, such as membrane markers, receptors or enzymes. For a fully documented desciption one may look at ${ }^{7}$. Cell image used with permission from http://www.siumed.edu/ dking2

As shown in figure 1, neutrophilic chromosomes are, apart from most other eukaryotic cells, typically accommodated in 3 to 5 interconnected lobes which are visible after, e.g. Hoechst stainings and share no direct similarities (size, geometry or amount of chromatin per lobe) among each other or to those of neighboring cells. ${ }^{2,9}$ The purpose of these unique shapes are still unknown as even chromosome-specific fluorescence in situ hybridization (FISH) showed solely random distributions along with a limited, but still existent, capability to synthesize proteins. ${ }^{1,10}$ Recent hypotheses suggest, however, that multiple, elongated nucleic compartments may benefit the cell to withstand even rough shear forces within the blood flow and improve the overall cell adjustment when it comes to migration or movement within close environments. ${ }^{8}$

The second neutrophil-specific feature involves the around 200-300 granules that are eponymous for its second name (granulocyte). ${ }^{11}$ These small, vesicular compartments are homogeneously distributed within the cytoplasm, function as a reservoir and mediator for a majority of cell function-related components and release their constituents upon particu- 
lar signaling cascades in an exocytotic fashion (including stimulation of membrane receptors, $\mathrm{Ca}^{2+}$ influx, and lipid remodeling, see also chapter 2.1.4). ${ }^{12}$ Histochemical stainings as well as high-resolution subcellular fractionation techniques identified up to now four distinct subtypes of these particles, which vary not only in size and release tendency but also in the function of their constituents as well as membrane-bound proteins. ${ }^{2,7,13}$ Here, primary granules (also named azurophilic granules due to their affinity to the basic dye azure A) define the largest and most common type of vesicles within the cell. ${ }^{1,13-15}$ Together with secondary, neutrophil-specific granules they also constitute the primary repository for antimicrobial proteins, such as myeloperoxidase (MPO), defensins or lactoferrin, as well as for a large number of reactive proteases including neutrophil elastase (NE), cathepsin G or collagenase. ${ }^{1,7}$ Further subpopulations usually vary in the amount and objective of their inlying particles. Yet, they are generally divided in either gelatinase-rich objects (tertiary/gelatinase granules) or those that do not contain any proteolytic enzyme but rather represent a reserve stock for various membrane and plasma proteins (secretory vesicles). ${ }^{2,16,17}$ For this reason, the fields of application a specific granule is involved in is highly diverse and can reach from migration-related membrane modifications up to the degradation of pathogenic material within anti-inflammatory cell responses (for an in-depth description one might consider examining Booregard et al. ${ }^{18}$ ). A list of their most essential cargo, as well as a graphical sketch of their geometrical properties and size relations, was furthermore added to figure 1.

In addition to these two quite perceptible features, neutrophils also exhibit a number of other intriguing characteristics: besides their average diameter of around $12-15 \mu \mathrm{m}$ during adhesion, or roughly $9 \mu \mathrm{m}$ while in suspension, they lack for example both, nucleoli and their rough endoplasmic reticulum; a circumstance that is rarely found within any other nucleated cell. ${ }^{11}$ Likewise, the size of their Golgi-apparatus is below-average $(\mathrm{d} \approx$ $600-800 \mathrm{~nm}$ ), and mitochondria and ribosomes can be found to a less extent than in other cells of the PMN family. ${ }^{2,11}$

\subsubsection{Neutrophil Development}

Aside from their morphology, the origination of most of the aforementioned components represents a likewise important topic, most notably as their features progressively entwine with the developmental stage of the cell during granulocyte production (granulopoiesis). ${ }^{2}$ Conventionally, neutrophils derive from pluripotential stem cells in the bone marrow, where, after multiple differentiation steps of associated colony-forming units (see figure 2 for more details), the first neutrophilic precursor cells (myeloblasts) are formed as well. ${ }^{1,19-21}$ Myeloblasts then follow distinctive maturation stages (including the formation of promyelocytes, myelocytes, metamyelocytes, band cells and finally mature neutrophils) in which, equally, specific parts of the neutrophilic intracellular environment are 


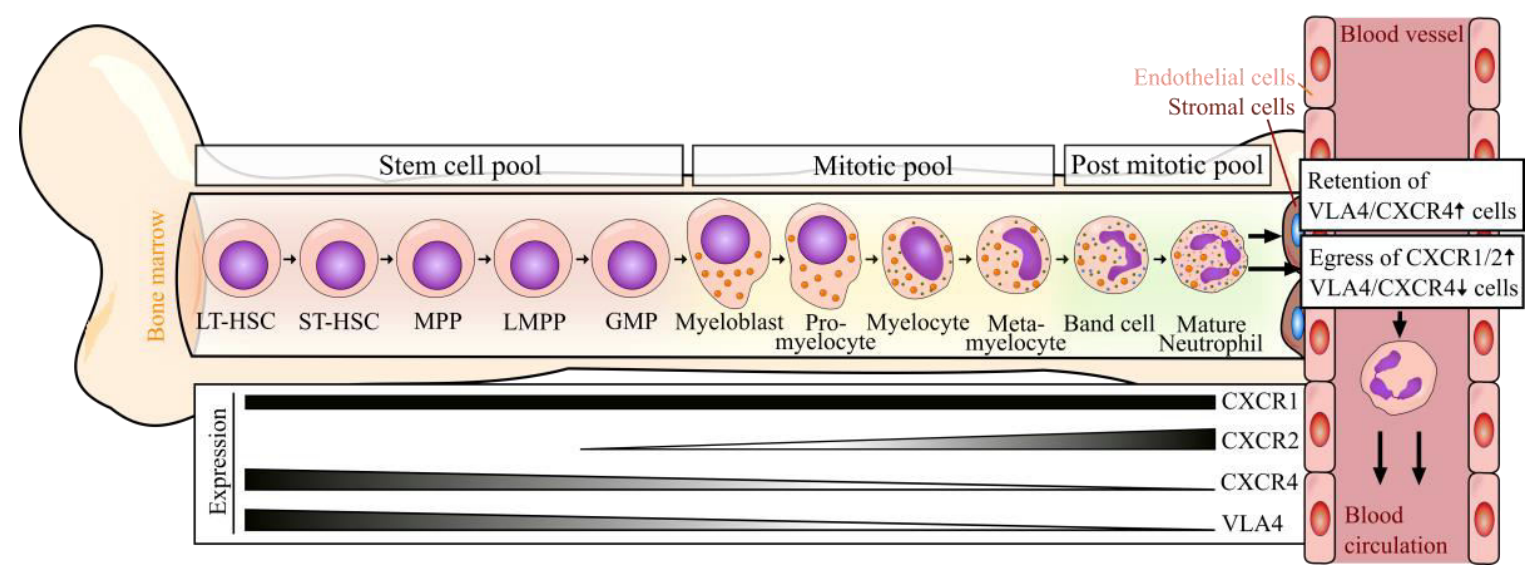

Figure 2: Neutrophil maturation in the bone marrow. The development of neutrophilic granulocytes can be visually divided in three different phases/pools. First, original stem cell differentiations lead to the creation of myeloblasts, first neutrophilic progenitor cells. Further developments from promyelocytes to metamyelocytes, band cells and finally matured neutrophils define not only necessary steps to gradually create neutrophilic features (granules, lobular nuclei) but also show consistent regulation of receptor proteins. Both, for example, CXCR4 as well as VLA4 are downregulated whereas CXCR2 is upregulated at the start of the mitotic phase. With this, retention of immature neutrophils is mediated via CXCR4/CXCL12 and VLA4/VCAM1 ligand-binding pathways by marrow endothelial and stromal cells leading to a regulated release of matured neutrophils to the blood circulation.

(Figure design adapted from ${ }^{19}$ and ${ }^{21}$ ).

gradually developed. The nucleus, for example, permanently changes its form from an initial, rather round shape at the myeloblast stage into a more and more banded-like stature and finalizes its transformation with the lobular geometries seen in matured neutrophils. ${ }^{20}$ Likewise, individual granules could only be identified after distinct maturation steps alongside with their corresponding transcription factors. ${ }^{2,22}$ Here, primary granules were first found at the myeloblast to promyelocyte stage, secondary granules at the myelocyte to metamyelocyte stage, tertiary granules appeared first in band cells and secretory vesicles, finally, were a product solely seen in mature neutrophils. ${ }^{2,20,23}$

Interestingly, this convergent evolution of proteins and intracellular compartments during maturation appears to be more than just a mere solution to circumvent evolutionary restrictions. Downregulation of distinct membrane receptors, such as CXC chemokine receptor type 4 (CXCR4) or the Very Late Antigen-4 (VLA4), for example, were also found to be deeply linked to regulation systems that monitor and control neutrophil release to blood. ${ }^{1,2,11}$ To explain this more contextually, neutrophils can normally leave the bone marrow and enter the blood circulation once they are matured. ${ }^{2,24,25}$ The number of exiting cells, however, stays tightly regulated (roughly to $10^{11}$ units per day or $1-2 \%$ of all cells produced) due to a series of membrane proteins (particularly C-X-C motif chemokine 12 (CXCL12) or vascular cell adhesion protein 1 (VCAM-1)) that are generated by surrounding endothelial/stromal cells and retain CXCR4/VLA4-containing objects by simple receptor/ligand binding reactions. ${ }^{20,26}$ Downregulation of these receptors during maturation thus enables the partial release of neutrophils while CXCR4/VLA4-deeply laden progenitor cells are kept within the bone marrow. ${ }^{5}$ In contrast, the induction of Granulocyte-Colony Stimulating Factor (G-CSF) by dendritic cells, as well as other growth factors, were shown to reverse these interactions again and increase neutrophilic 
release in times of need. While G-CSF itself is known to reduce CXCL12 expression and upregulates the development of complement ligands on different cell types, other factors, such as stromal derived factor-1 (SDF-1), decrease neutrophilic CXCR4 expression emphasizing the development of neutrophils as an efficient yet also tightly implemented machinery for immunologic purposes. ${ }^{27-32}$

\subsubsection{Migration and Mechanical Properties}

Once in the blood vessels, the metabolism of circulating neutrophils is primarily dictated by the enormous number of biochemical and - physical stimuli that are exerted throughout the entire vascular system. The outcome of each of these interactions is, likewise, diverse and can range from small intermolecular changes within the cytoplasm up to formations of entirely new phenotypical properties. However, whether or not a corresponding stimulus may affect the cellular metabolism depends strongly on the interactions with dedicated receptor units as well as their ability to prime the cell for upcoming functions. ${ }^{2,25,33-36}$ At this point, "cell priming" can be described at best as a process of enabling the functional response of a neutrophil to external influences and is usually followed by a subsequent "activation" event which triggers intracellular signaling cascades and initiates anti-inflammatory operations. ${ }^{37,38}$ Interestingly, neutrophils in the blood vessels (or in vitro) are typically found quite quiescent, also known as in the resting state, at least as long as no suitable stimulus can be found in their proximity. In this state, they usually remain completely round, own a smooth plasma membrane and are barely able to synthesize proteins or interact with their external environment. ${ }^{24,25}$ Primed or activated cells, on the contrary, are known for their directly opposite behavior: typically described with an amoeboid morphology, they are able to spread and migrate on biofilms and other biological substrates, synthesize a large number of proteins and were shown to exert an increased production of reactive oxygen species (ROS), a hallmark of all activated PMNs. ${ }^{39-41}$ Ways of priming a neutrophil can likewise come in different shapes and sizes as both in vitro and in vivo studies documented activation by diverse physicochemical sources including cytokines (e.g., interleukin 1 alpha (IL-1 $\alpha$ ) or tumor necrosis factor alpha (TNF- $\alpha)$ ), antigens (e.g., pathogen endotoxins), but also mechanical stimuli such as glass or distinct biofilms..$^{25,42-44}$

Due to this, every circulating neutrophil shares a dichotomous fate: in the absence of any specific stimuli, the cells' life consist of a pure flow-driven motion through vessels of the vascular system until, after a rather short life cycle of $6-12$ hours, they may return to the bone marrow and become degraded by macrophages and other cells of the resident immune system..$^{2,25,45,46}$ In case of damaged tissues, inflammation or other stimuli, however, intrinsic signaling cascades of the immune system can also activate neutrophils, force 


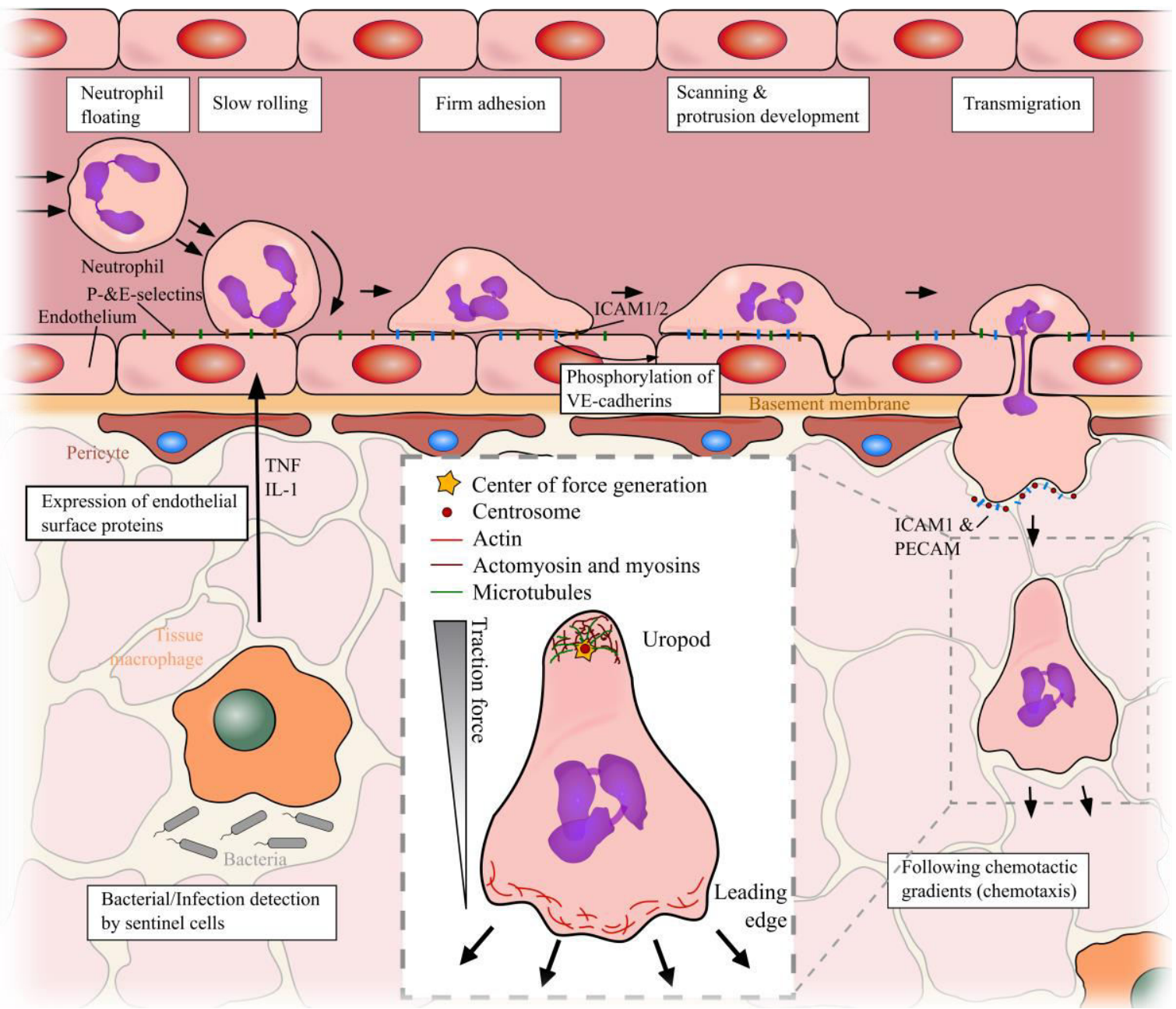

Figure 3: Neutrophilic diapedesis and migration. Depending on external stimuli, neutrophils either flow inactively through the bloodstream and head back to the bone marrow or become activated and crawl through the endothelial wall to the associated inflammation/damaged site. The latter normally starts with a proinflammatory trigger activated by macrophages, mast cells and other sentinel cells, which leads to a release of danger signals/mediators effecting surrounding endothelial cells and starts a leucocyte adhesion cascade. Here, first, nearby endothelial cells initiate the expression of specific surface molecules, such as P-and E-selectins, and force close neutrophils to attach via ligand bindings on neutrophilic surface glycoproteins. Subsequent development of ( $\beta 2$-)integrins then changes the neutrophilic migration dynamics from slow rolling to firm adhesion and primes the cell for following transmigration processes. As a consequence, neutrophils become amoeboid-shaped and develop a controlling front (leading edge) and an attachment and motor unit at the rear (uropod) due to polarized cytoskeletal polymerization. Following that, further stimuli give rise to the generation of pseudopodia, elongated cell extensions at the neutrophils front, that search for permissive sites on the endothelium and infiltrates the tissue through weakened cell junctions. Further migration, mediated by neutrophilic and endothelial adhesion molecules as well as several chemokines, lead to the directed penetration of the endothelium, basement membrane, pericyte layers and other biological hurdles and guide the cell to the associated inflammation site. (Middle picture adapted from ${ }^{8}$ ).

them to transmigrate through the endothelial wall (diapedesis) and steer their prospective antimicrobial actions to eliminate potential pathogens or other foreign particles (see also figure 3). The first step of this prolonged mechanism normally includes the mobilizations of neutrophils through a process known as the leukocyte adhesion cascade. ${ }^{47}$ Primarily, endothelial cells of blood vessels close to an affected site become activated by compounds such as histamine or thrombin (among others released as an inflammatory response by mast cells or macrophages) and express a number of adhesion receptors (e.g., E- and P-selectins) that are able to bind on neutrophilic membrane proteins and cause a slow rolling of the cells. ${ }^{1,48}$ Subsequently, chemokines, similarly expressed by immune cells close to the inflammation site, prime the cell and induce the assembly of several 
integrins (mainly of the $\beta 2$-subset) which, again, bind to endothelial intercellular adhesion molecules-1 and -2 (ICAM-1 \& ICAM-2) and enforce a firm adhesion of the corresponding neutrophil. After this point, morphological changes of the leukocyte are, due to its priming, clearly visible and lead to a predominant cytoskeletal polarisation at the rear and the front of the cell. ${ }^{8,25}$ The same polarisation then also enables the development of so-called pseudopodia, elongated cell extensions at the cellular front, that search for permissive sites on the endothelial wall and thus initiate the transmigration towards the inflamed tissue. ${ }^{49}$

Intriguingly, it is still not completely understood how a neutrophil then transmigrates through the endothelial tissue as both, the mechanical way of how the cell progresses and the molecular interaction with endothelial cells, are parts of an ongoing debate. Tractionforce microscopy of moving neutrophils showed so far that these cells characteristically move in an amoeboid fashion; a process that utilizes the above mentioned cytoskeletal polarisation to create self-contained front and rear environments and enables a "squeezing"-like motion to move forward. ${ }^{50}$ In more detail, within this process dynamic actin deand polymerization mechanisms give rise to two specific protrusions within the cell: a leading, pseudopodia containing edge, that is able to sense external stimuli and orientates the cellular body accordingly, as well as an extended rear, the uropod, which functions as a contractile motor and attachment point during locomotion. ${ }^{8,24,50}$ The general migration pattern then starts with the alignment of the leading edge, which, predominantly driven by feedback loops between corresponding membrane receptors and the actin-cytoskeleton, react to present stimulations (e.g., chemokines or physical properties of its environment) and develop new extensions towards the reactive side. Subsequently, once the leading edge is oriented, contractile forces generated by the actomyosin meshwork at the rear push the cell forward by squeezing cell contents towards the front and diminishing the uropod's adhesion at the same time..$^{50-52}$ Due to this efficient process, neutrophils were shown to be one of the fastest moving cells with an average velocity of $19 \pm 6 \mu \mathrm{m} / \mathrm{min}$ in vitro - around three times faster than any other leukocyte (T lymphocytes: $7 \mu \mathrm{m} / \mathrm{min}$ ) and up to a hundred times faster than mesenchymal cells such as fibroblasts or cancer cells $(\approx$ $0.2-1 \mu \mathrm{m} / \mathrm{min}) .{ }^{53-55}$ Recent studies suggest, in addition, that the position and structure of the nucleus significantly contribute to these highly migratory properties as it is able to stabilize the cell's front-rear axis and acts as a force propagator for the above-mentioned contraction forces. ${ }^{56}$

Their fast movement set together with leukocyte-endothelial cell adhesion interactions also aids these cells during the transmigratory process in vivo. Here, the aforementioned, firm ICAM- 32 -integrin bindings normally trigger an intercellular signaling cascade of nearby endothelial cells first, which ultimately leads to phosphorylation of vascular endothelial cadherins (VE-cadherin) and a loosening of otherwise tight cell junctions 
within the endothelial cell layer. ${ }^{57,58}$ Neutrophils then migrates through these opened cell junctions via sequential interactions with several membrane proteins and adhesive molecules (particularly junctional adhesion molecule-A and -C (JAM-A/C) and platelet endothelial cell adhesion molecules (PECAM)) while various chemokine gradients orientate its body towards the inflamed tissue (chemotaxis). ${ }^{58-62}$ Further, more recent studies also provided information on a second, transcellular migration type that was found to occur either in response to special chemokines (e.g. formyl-Met-Leu-Phe (fMLP)) or in case of hardly accessible junction sites (as for example at the blood-brain barrier) and included the formation of transcellular channels that transported neutrophils while leaving associated gap junctions intact. ${ }^{58,63-65}$ Finally, once a neutrophil then passed through the endothelium, it further infiltrates pericytes and the vascular basement membrane in a likewise ICAM-1/PECAM dependant manner before it chemotactically approaches the associated inflammation site and starts its anti-inflammatory operations. ${ }^{58,66,67}$

\subsubsection{Immunological Response Functions}

Once at the area of infection, neutrophils contribute to the immune system by a vast range of antimicrobial mechanisms and cell-cell interactions. Normally, first "pioneer" cells reach the associated tissue and amplify chemotactic effects of already existing chemokines resulting in a process called "neutrophil swarming", a secondary, long-range attraction cascade that recruits even more leukocytes and usually lead to vast agglomerations at the point of inflammation. ${ }^{68}$ The specific communication pathway of this process is still unknown as it was discovered only recently. However, first mice studies revealed that lipid attractant leukotriene B4 (LTB4) might play a vital role within this mechanism, in particular as LTB4r $1^{-/-}$neutrophils were shown to be unable to generate swarming patterns anymore. ${ }^{69}$

Meanwhile, resident neutrophils start to purge their surrounding or, respectively, clear the tissue from pathogenic particles or cell residues by one (or more) of the following mechanisms:

\section{Phagocytosis}

First depictured a century ago, phagocytosis describes the ability of neutrophils and other phagocytes to engulf pathogenic material (e.g., bacteria or cell residues) and form an intracellular compartment, the phagosome, in which degradation by proteolytic enzymes and reactive oxygen species (ROS) leads to an effective elimination of the internalized object. $^{70-73}$ The process normally starts with the identification of the pathogen via distinct membrane recognition receptors (among others, receptors of the pattern recognition receptor family (PRR), like Toll-like receptors (TLR), as well as Fc-receptors (FcR) such as 
Fc $\gamma$ RIIA or Fc $\gamma$ RIIIB), which, while in contact with pathogen- or damage-associated molecular patterns (PAMP or DAMP) on the objects surface, become activated and initiate phagocytosis-specific signaling cascades (see also figure 4). ${ }^{70,74-76}$

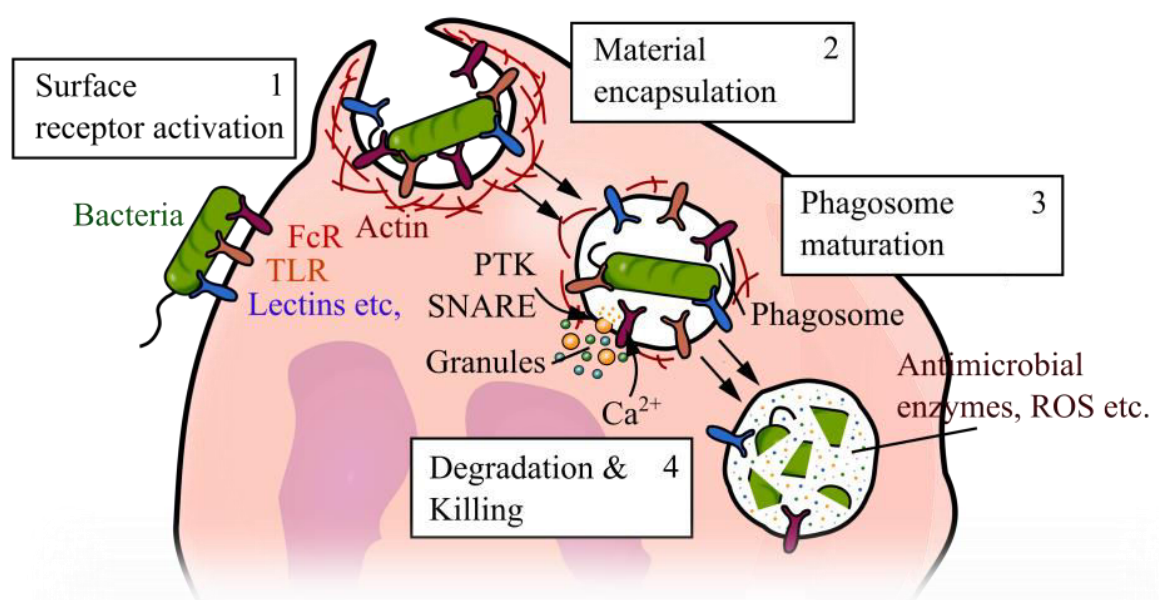

Figure 4: Schematic represantation of phagocytosis. Upon contact with PAMPs or DAMPs on the surface of detrimental objects (bacteria, cell residues etc.), specific neutrophilic surface receptors become activated and initiate the phagocytic process. Starting with a rapid, vast cytoskeletal polymerisation at the corresponding surface area, neutrophils first build pseudopodia around the object, encapsulate it within minutes and build a so-called phagosome that is imported into the cells body. Subsequently, increased levels of $\mathrm{Ca}^{2+}$ reduce the concentration of built actin again, induce an enrichment of receptors on the phagosome and instruct insertion of granular compartments by PTK/SNARE mediated granule fusion (phagosome maturation/phagolysosome). As a consequence, introduced proteolytic enzymes or ROSs are able to degrade the object and successfully purge the affected inflammation site.

Following downstream signals, involving phosphorylation of various proteins mediated by and with lipid kinases, then start to induce actin polymerization and localized membrane remodeling which form and fuse pseudopodia around the pathogen until it is fully encapsulated and trapped within the built vacuole (phagosome) ${ }^{70,77}$ In this state, the phagosome's membrane is continuously enriched by additional receptor units while induction of supplemental $\mathrm{Ca}^{2+}$ to the cytosol initiate the degradation of the circumjacent cytoskeleton again. ${ }^{70,73}$ Subsequent feedback loops, also originated by the activation of the aforementioned receptors, then starts to fuse intracellular granules with the phagosome in a $\mathrm{Ca}^{2+}$ concentration-dependent manner (including granule transport by localized cytoskeleton/tubulin polymerization and mediated fusion by protein tyrosine kinase (PTK) and soluble N-ethylmaleimide-sensitive-factor attachment receptors (SNAREs)) and thus introduces their antimicrobial content to the pathogen (phagosome maturation/phagolysosome) ${ }^{70,78-80}$ Following this, the final degradation step may appear in two different ways: either it is fulfilled by a large number of proteolytic enzymes already mentioned in chapter 2.1.1 or by the development of free reactive oxygen species built within the phagolysosome. ${ }^{72,78}$ In doing so, the latter mainly takes place after induction of nicotinamide adenine dinucleotide phosphate (NADPH) oxidase which is able to promote the electron transfer of cytosolic NADPH to oxygen and consequently produces free superoxides around the pathogen (respiratory burst, see equation 1). ${ }^{70,72}$ These, on the other 
hand, can be further converted to other, more persistent oxygen metabolites such as hydrogen peroxide or hypochlorous acid. ${ }^{70}$

$$
\mathrm{NADPH}+2 \mathrm{O}_{2} \rightleftarrows \mathrm{NADPH}^{+}+2 \mathrm{O}_{2}^{-}+\mathrm{H}^{+}
$$

Intriguingly, even though the general description of phagocytosis and its underlying pathways have been studied for an extensive amount of time now, quite less is known about its dynamic properties as well as the conditions and consequences on which a specific object is uptaken. ${ }^{81,82}$ Interestingly, it seems that both, associated receptors react not only to biochemical but also topological, electrical or even other surface dependant cues and that the dynamics of the phagosome maturation depends on the composition but also the size and geometry of the ingested material. ${ }^{81,83-88}$ These new insights could be of high relevance, especially in regards to cellular drug delivery or transport systems, and will be covered more specifically within one of the upcoming discussion section (see chapter 5.3).

\section{Extracellular Degranulation}

Similar to the fusion process during phagocytosis, neutrophils are also able to release granular components to the extracellular matrix (extracellular degranulation). Here, the associated signaling pathways do not differ significantly from those described in the last section. Upon activation of local membrane receptors, again including FcRs and TLRs but also adhesion molecules such as selectins, granules are transported by gradual polymerization of the inlying cytoskeleton while local $\mathrm{Ca}^{2+}$ enlargements in combination with SNARE complexes and kinase-mediated phosphorylation cascades enable a controlled membrane fusion and release process. ${ }^{89}$ In contrast to intracellular degranulation during phagocytosis, however, the release of primary or secondary granular content was reported to occur comparably seldom and seems to be incumbent upon various $\mathrm{Ca}^{2+}$-dependent signaling pathways and receptor activations. ${ }^{90}$ Vice versa, enhanced granule liberation was found in case of leaking phagosomes, e.g. when engulfed objects were too big or misshapen to be internalized completely (frustrated phagocytosis). ${ }^{73,90}$ Furthermore, aside of its antimicrobial mechanisms, extracellular degranulation was also demonstrated to apply various cell supporting functions. ${ }^{90,91}$ Fusion of secretory vesicles, for example, was found to increase the overall appearance of adhesion and chemotactic receptors like formyl peptide receptor 1 (FPR1) and thus promote neutrophil migration and recruitment. ${ }^{90}$ Likewise, the release of secondary and tertiary content, especially the enzyme collagenase, was proven to facilitate diapedesis by local degradation of collagen during basement membrane infiltration. ${ }^{90,92}$ 


\section{Neutrophil Extracellular Traps (NETs)}

As a third mechanism, the generation of neutrophil extracellular traps (NETs) displays the latest gear of neutrophils antimicrobial machinery and, at the same time, one of the center points addressed in this thesis. First described in 2004, its origin derives from early studies of Brinkmann and colleagues who exposed neutrophilic granulocytes with the tumor promoter phorbol-12-myristate-13-acetate (PMA), as well as the chemokine interleukin-8 (IL-8), and experienced an unprecedented type of cell death: apart from common apoptosis or necrosis pathways, neutrophils ended their life by releasing massive web-like structures, comprised of chromatin and different antimicrobial granule constituents, which were able to do both, trap and degrade, diverse types of bacteria and hence were nicknamed NETs. ${ }^{93}$ Now, 15 years later, neutrophil research found a renaissance as the generation of NETs via neutrophil death (NETosis) could be associated with a large number of immunological mechanisms including the incapacitation of diverse pathogens but also the aggravation of several immune-specific diseases, such as thrombosis or lupus erythematodes. ${ }^{94-96}$ This is why, especially in the last years, the characterization of NETosis has caught the attention of many researchers worldwide.

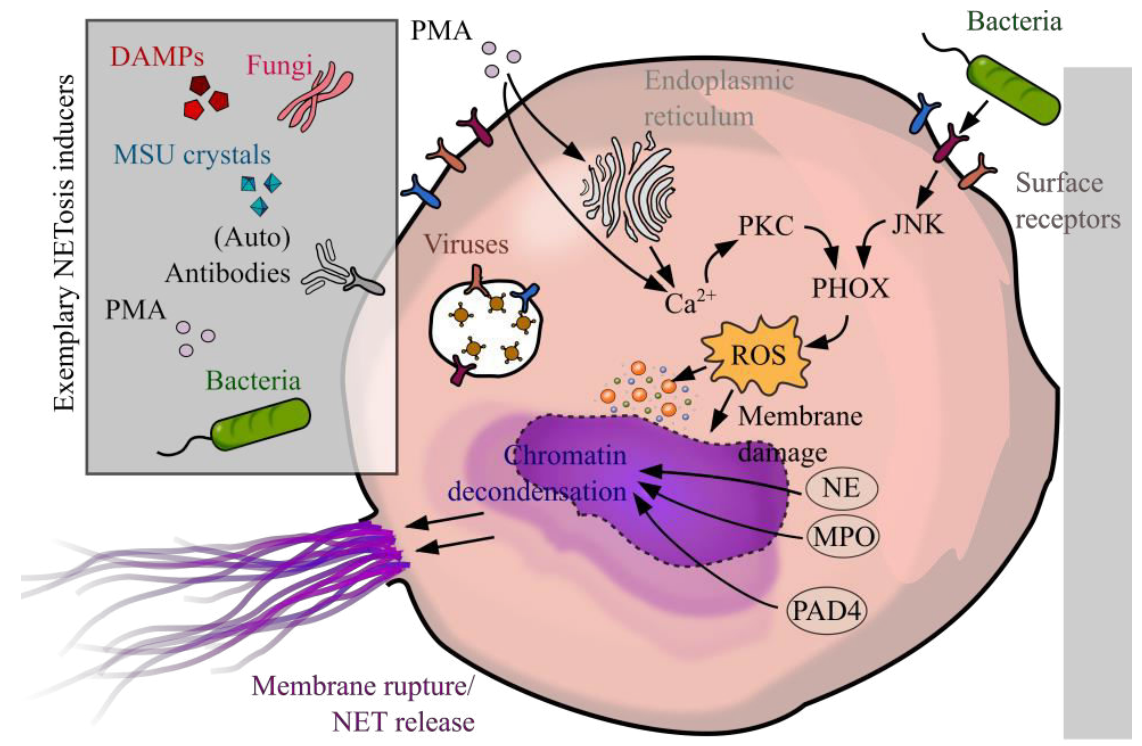

Figure 5: Exemplary pathways of NETosis. Micro-organisms, various DAMP/PAMP containing objects (MSU crystals, bacteria, fungi etc.), viruses or (auto)antibody reactions showed a NETotic effect via several signalling cascades and effector proteins. Upon activation of PMA (left side), for example, increased intracellular $\mathrm{Ca}^{2+}$-levels from the ER were reported to enhance PKC activity and thus induce NADPH oxidases to congregate into functional complexes (PHOX). Such complexes then form intracellular ROS and cause granular and nuclear membrane rupture resulting in the accessibility of the nuclear content. Granular NE, MPO and PAD4 then translocate into the nucleus and apply histone deimination followed by the characteristic intracellular chromatin decondensation. Subsequently, the DNA-meshwork mixes with the anti-inflammatory, cytosolic content and is released as NETs after cellular membrane rupture. Here, ways of ROS-generation differ depending on the stimulus. LPS from gram-negativ bacteria (right side), for instance, were shown to generate NETs independent from PKC activation but utilize a TLR4-mediated JNK pathway. Likewise, even ROS-independent pathways are discussed recently.

As a natural consequence, the insights about its general pathway and possible key players have grown since then. Beside PMA and IL8, additional activation sources were found, 
among others, several types of fungi, bacteria, algae, antibody-antigen-complexes, autoantibodies or tumor necrosis factors (TNF). ${ }^{97-100}$ Upon ligand binding on several membrane receptors, again predominantly driven by TLRs and receptors of the IgG-Fc family, these substances are thought to activate specific signaling cascades that, each by its own, result in damage of vital, intracellular structures and evoke a characteristic chromatin swelling, the hallmark of all NETosis pathways (figure 5). Common activation by Phorbol-12-myristate-13-acetate (PMA), for example, was found to stimulate the endoplasmic reticulum first leading to increased intracellular $\mathrm{Ca}^{2+}$ levels and the downstream activation of protein kinase $\mathrm{C}$ (PKC). ${ }^{93,100-102} \mathrm{PKC}$, in turn, phosphorylates the heme binding subunit (gp91phox) of intracellular NADPH oxidase (NOX) and ultimately leads to the generation of ROS within the cellular body (NOX-dependent pathway). ${ }^{103,104}$ Other inducers, such as Lipopolysaccharide (LPS) by gram-negative bacteria, also resort to NOX-activation but translate their signal by different enzymes like TLR promoted c-Jun N-terminal Kinases (JNKs) and thus give rise to various, submechanical differences within the NETotic phenotype (see also chapter 5.3). ${ }^{105}$ Meanwhile, due to the generation of ROS, the physical integrity of the cells and its compartments starts to slowly disintegrate. Oxidation of major membrane compounds, especially of nuclear and granular lipid-units, begin to be destabilized and primes the fusion of cytoplasmic, granular and nuclear contents. As a consequence, NE and MPO, generally stored in primary granules, are able to head to the nucleus and degrade linker and core histones as well as enhances chromatin decondensation in combination with citrullination processes mediated by peptidyl arginine deiminase 4 (PAD4). ${ }^{97,106-108}$ This process then leads to the swelling of the cellular chromatin mentioned above, which, after merging with the now antimicrobial, cytosolic content and an additional rupture of the cellular membrane, is released extracellularly as NETs. ${ }^{106}$

Intriguingly, besides varying upstream signals for NADPH-oxidase activation, several NOX-independent pathways have also emerged in recent years adding new insights into this vast and complex immunological process. Physiological agonists, such as TNF $\alpha$ for example, were found to operate even during inhibition of PKC or JNK ${ }^{109}$ leaving the question whether such molecules avail themselves of other ROS-sources ${ }^{110}$ or produce NET-formation by completely independent pathways. In addition to that, less is known about the signals upstream of PAD4 or the reason why the cellular membrane ruptures in the end, with the latter being one of the focus points of this thesis (see also chapter $3.1 \&$ 5.1.2). Several studies challenged moreover the general conception of NETosis leading to ultimate cell death as latest findings also observed NET formation from mitochondriaderived DNA with cells showing intact and functional behavior after release (vital NETosis). ${ }^{11,112}$ 
Consequently, the current research state of NETosis reached an interesting level of knowledge. While so far several chemical driving factors could be identified enabling a general perception of this novel, immunological mechanism, many of its underlying interactions still remain indeterminate. All the more, interdisciplinary investigations as presented in chapter 3.1 and 3.3, become essential to elucidate the process of NETformation in all of its aspects.

\section{SUMMARY OF CHAPTER 2.1}

Neutrophilic granulocytes are a unique type of leukocytes and the first line of defense of the mammalian immune system. Normally recognized by their vast number of granules and their multi-lobular nucleus, they rise from the bone marrow by sequentual differentiation of myeloblast based precursor cells and release into the bloodstream after maturation. There, they either become activated by external stimuli or retreat to the bone marrow where they are degraded by resident macrophages. Inflammation-based activation occurs then in three steps: neutrophils become primed by endothelial adhesion molecules and start to adhere at the endothelial surface first. Chemotactic factors then guide them through the cell tissues while they utilize a fast and efficient amoeboid movement. Once at the inflammation side, they are able to recruit more leukocytes and perform antimicrobial functions (phagocytosis, degranulation, NETosis).

For more, general information about this topic, following reference works have especially proven to provide an useful introduction: ${ }^{5,8,18,22,23,48,79,94,108,112}$

\subsection{Single-walled Carbon Nanotube Nanosensors}

While neutrophilic granulocytes and the characterization of its antimicrobial processes define one central aspect of this thesis, the utilization of single-walled carbon nanotube (SWCNT) based nanosensor in biological systems plays definitely another - not least due to the studies presented in chapter 3.2 and 3.3 utilized their unique optical and biocompatible properties for diverse biological applications. Therefore, the next sections will briefly describe the overall features of SWCNTs and summarize their basic principles including optical characteristics, functionalization and imaging methods.

\subsubsection{Structural and Optical Properties of SWCNT}

Over the years, improvements in nanobiotechnology facilitated a wide range of innovative opportunities to sense, image or treat biological systems, especially on a molecular level. ${ }^{113,114}$ Vast numbers of diverse studies and research topics already underlined the broad spectrum of applications that resultant new materials, such as nanoparticles, 
quantum dots, -wires and carbon nanotubes, can offer while their size, ease of modification and optical properties define them (theoretically) as a near-perfect tool for any optical or electronic application. ${ }^{115-121}$ On this basis, especially carbon nanotubes gained much attention in recent years as novel fabrication and functionalization methods made them not only commercially accessible but also a well-defined basis for single-molecular biosensor production, drug delivery and even phototherapy. ${ }^{122-128}$ Here, the reason for this wide range of possible applications actually originates in their simple, yet unique, structure: nanotubes are, above all, allotropes of carbon and generally composed of symmetrically stacked, modifiable $\mathrm{sp}^{2}$-hybridized carbon atoms that are cylindrically arranged and thus form tubes of $0.4-2 \mathrm{~nm}$ width and typically several hundreds of nanometers length (figure 6a-b). ${ }^{129-132}$

a
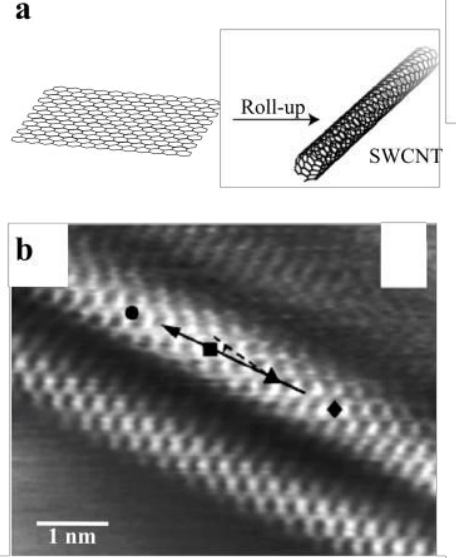

e

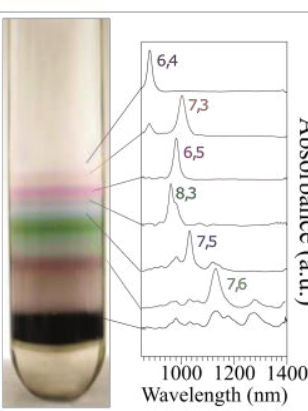

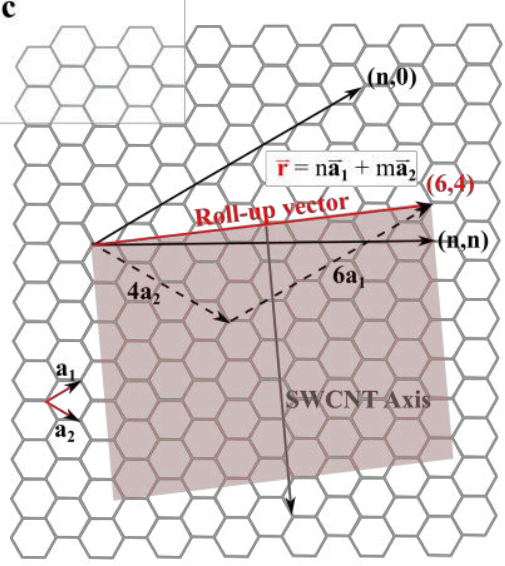

d

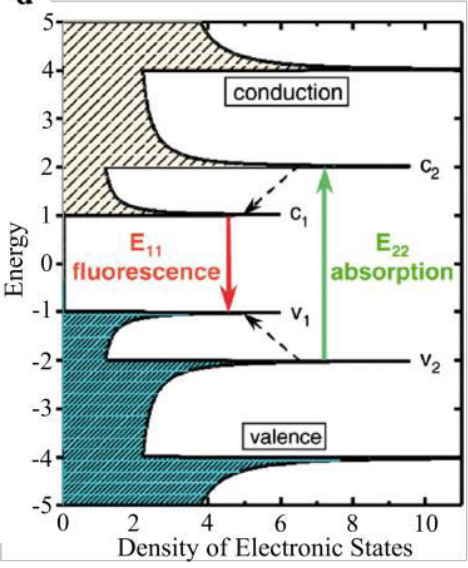

g
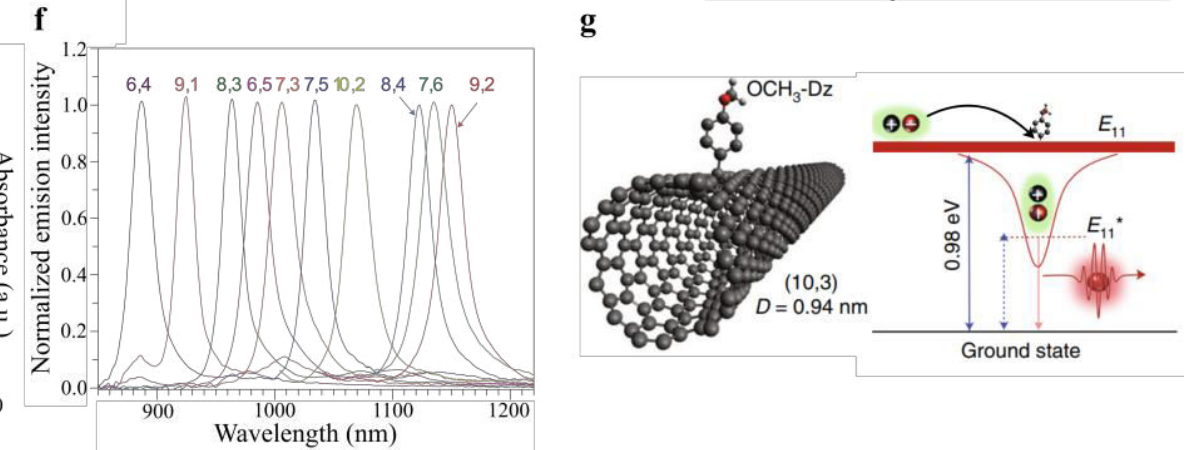

Figure 6: Structure and optical properties of SWCNTs. a-b General structure of a single-walled carbon nanotube (SWCNT). Figuratively, it can be visualized as a rolled-up graphene layer (top) and thus consists of a homogeneously stacked carbon $\mathrm{sp}^{2}$-orbital lattice (STM image extracted from ${ }^{132}$ ). $\mathbf{c}$ Geometrical description of a SWCNT chirality. The structure of a $(\mathrm{n}, \mathrm{m})$-nanotube is defined by the roll-up vector $\vec{r}=n \overrightarrow{a_{1}}+m \overrightarrow{a_{2}}$ on which the graphene lattice is convoluted. Here, $\overrightarrow{a_{1}}$ and $\overrightarrow{a_{2}}$ constitute the lattice unit vectors of the environment. d Density of electronic states of a semiconducting SWCNT. Depending on the chirality of the nanotube, different electronic states can be found and thus lead various band gaps between the valence band and the conduction band. For SWCNTs, normally, the $\mathrm{E}_{22}$-absorption/ $\mathrm{E}_{11}$-emission mechanism defines the characteristic optical properties of the corresponding material (image reproduced from ${ }^{139}$ ). e-f Resulting absorption and emission spectra of different $(\mathrm{n}, \mathrm{m})$-SWCNTs (image reproduced from ${ }^{140}$ ). $\mathbf{g}$ Depiction of a nanotube defect and its effect on exciton diffusion. Due to the local alternation of the nanotubes electronic structure, the energy of a transpassing exciton can be affected and changes the optical output of the SWCNT (image reproduced from ${ }^{143}$ ).

As such, the change in diameter is a result of different subspecies that arise by varying "folding"-angles, so-called chiralities, on which a single-layer nanotube (single-walled carbon nanotube, SWCNT) can be formed. Figure 6c depicts this process schematically. As a standard convention, the chirality of an $(n, m)$ SWCNT is denoted by the length $n$ 
and $\mathrm{m}$ in the direction of the lattice unit vectors $\overrightarrow{\mathrm{a}_{1}}$ and $\overrightarrow{\mathrm{a}_{2}}$ of which the rolling vector $\overrightarrow{\mathrm{r}}=$ $n \overrightarrow{a_{1}}+m \overrightarrow{a_{2}}$ is composed of. Hence, the diameter of a specific chirality changes due to different connection points the lattice has to combine to form a tube (one may even calculate its value by the formula $d=\frac{a}{\pi} \sqrt{\left(n^{2}+n m+m^{2}\right)}$ with a $=0.246 \mathrm{~nm}$ being the unit lattice length). ${ }^{115,133}$

\begin{tabular}{lcc}
\hline Conductivity state & Chirality condition & Electronic structure \\
\hline Metallic & $\mathbf{n}-\mathbf{m}=\mathbf{0}$ with $\mathrm{n}, \mathrm{m} \in \mathbb{N}$ & No band gap \\
& $\mathbf{n}-\mathbf{m}=\mathbf{3} \mathbf{x}$ with $\mathrm{x}, \mathrm{n}, \mathrm{m} \in$ & $\begin{array}{c}\text { Small band gap } \\
(\approx 1-200 \mathrm{meV})\end{array}$ \\
Semimetallic & $\mathbb{N}$ and $\mathrm{x}>0$ & \\
& Any other comb. of $(\mathbf{n}, \mathbf{m})$ & $\begin{array}{c}\text { Large band gap } \\
(>0.5 \mathrm{eV})\end{array}$ \\
Semiconducting $\mathrm{with}, \mathrm{m} \in \mathbb{N}$ & $(>0.5$ \\
\hline
\end{tabular}

Table 1: Electronic properties of (n,m)-nanotubes. Depending on the chirality of the given nanotube, its electronic and thus optical behavior changes according to the above mentioned classifications (table reproduced from ${ }^{115}$ ).
Intriguingly, not only the size of an SWCNT was found to change with its chirality but also its conductivity, and thus its optoelectronic properties, which makes them, especially for biosensor production, a versatile and valuable tool. Here, depending on the $(n, m)$ values, the common band gap of a nanotube can vary from 0

to about $2 \mathrm{eV}$, meaning their electrical conductivity can either show metallic, semimetallic or even semiconducting behavior. ${ }^{133}$ In general, this relationship can be explained by the different electron symmetries an SWCNT exhibits along its main axis while its first analytical description was pronounced by Hiromichi Kataura in $1999 .{ }^{134}$ His exact calculations between the band gap energy and the associated (n,m)-numbers (Kataura plots) may exceed the actual scope of this thesis, however one might derive a rather simple, chirality-dependent correlation from it that may help to visualize given dependencies within the system (table 1). ${ }^{134,135}$ This then, in combination with the quasi-onedimensional surface SWCNTs exhibit, also paved the logical way for nanotube-based optical sensors as unidimensional lattice structures normally accompany distinct densities of electronic states and thus allow for optical transitions (van-Hove singularities). ${ }^{136,137}$ In fact, semiconducting SWCNTs do exhibit fluorescence light in the near-infrared region (nIR, $900-1600 \mathrm{~nm}$ ) due to sharp energy levels between the valence and the conduction band. ${ }^{138}$ Here, the most occurring transition were described by the $\mathrm{E}_{22}$-absorption/E11emission process in which, similar to common, organic fluorophores, the energy of a corresponding lattice electron is excited by the interaction of one (or multiple) photons $\left(\mathrm{v}_{2} \rightarrow \mathrm{c}_{2}\right)$ and is lost again by simple relaxation processes and the emission of a subsequent low-order photon $\left(\mathrm{c}_{1} \rightarrow \mathrm{v}_{1}\right.$, see also figure $\left.\mathbf{6 d}\right) .{ }^{115,138}$ As a result, every semiconducting $(\mathrm{n}, \mathrm{m})-\mathrm{SWCNT}$, with its own geometrical structure and corresponding band gaps, also exhibit its own unique and characteristic fluorescence signal (figure 6e-f). ${ }^{139,140}$

Naturally, this ability alone is a valuable property (and found already many scientific applications) but does not distinguish an SWCNT from other fluorophores regarding its 
sensor-building effects. In fact, the true advantage of SWCNTs can be seen in the excitonic nature of their excited electrons. Briefly, whenever a photon is absorbed by one of its lattice electrons (and thus excites it into the conduction band), it also delocalizes the electron marginally and creates a subsequent, positive charged "hole" at its former position. ${ }^{141}$ This generated electron/hole pair, also known as an exciton, is stabilized by repulsive Coulomb forces of surrounding lattice electrons and remains resilient as long as the excited electron stays in the corresponding conduction band. ${ }^{141-143}$ Similar to Försterresonance energy transfers (FRET), it is, however, also able to relay its state to electrons of neighboring carbon atoms resulting in an apparent diffusion of the quasiparticle. ${ }^{115,144,145}$ This migration-like process, in return, turns SWCNT surfaces into a viable tool for sensor productions as moving excitons can be influenced via perturbations or structural changes, e.g. of locally adsorbed molecules, and thus are able to modify the overall photomechanical state of the nanotube in response to external effects (see also figure $\mathbf{6 g}$ ). ${ }^{115,122,146,147}$

In addition to that, their extraordinary stable structure, in combination with the aforementioned optical features, produces even more arguments that highlight the significance of SWCNT-based biosensors, especially with regard to in vivo applications. Under biological conditions (room temperature, aqueous environments, $\mathrm{pH}$-values, etc.), for example, they don't blink or bleach and the emitted wavelength of most of their substructures lies within the so-called "tissue transparency window", an nIR-territory in which the optical absorption of biological tissues becomes minimal. ${ }^{148-153}$ SWCNTs furthermore exhibit strong and characteristic Raman signatures, possess a large Stokes shift and are, to a large extent, thermally stable and chemically inert. ${ }^{148,154,155}$ Their biggest drawback, on the other side, can be probably linked to their fairly low quantum yield (QY) in aqueous environments $(\approx 0.1-1 \%) .{ }^{156-158}$ Even though individual studies reported optimization processes with results of up to $30 \%$, the general way of how to improve the brightness of SWCNTs is still part of ongoing discussions. ${ }^{159,160}$

\subsubsection{Functionalization Methods \& Recognition Strategies of SWCNTs}

Now, to create a precise and functional biosensor, two components are of paramount importance: a specific recognition unit to interact with the sought analyte/molecule and a signal transduction entity that is able to convert the resulting interactions into a quantifiable signal. ${ }^{115,161}$ In the case of SWCNT-based optical sensors, the latter is naturally typified by the nanotube, or rather its aforementioned features, while its transduction signal can be either a change of its optical properties (e.g., de-/increase of intensity or signal wavelength) or of its intrinsic, electronic behavior (alteration of conductive capacity/ band gap shift, etc.). ${ }^{14,161}$ The generation of the recognition unit, in contrast, is generally accompanied by well-defined surface modifications since bare nanotubes, with their aro- 
matic, non-polar substructure, exhibit only minor interaction potential within biological compounds. ${ }^{115,162,163}$ Hence, a good SWCNT sensor design is equivalent to successful SWCNT surface functionalizations and thus relies on several conditions. Firstly, it needs to generate particular interaction sites on the SWCNT surface, the so-called corona, which, at best, interact solely with the sought analyte and can modify one (or more) of the nanotubes signaling options. ${ }^{114,115,123,164}$ Secondly, it has to adjust the resultant sensor properties according to the experimental needs, meaning not only the solubility but also the binding/dissociation dynamics, mechanical stability or lifetimes have to match to the corresponding environment of interest. ${ }^{165,166}$

With that in mind, both, functionalizations using covalent and non-covalent modification techniques were reported in the last years, each of them showing their own, characteristic assets and drawbacks (for a more detailed description see one of the following reviews). ${ }^{15,167-171}$ Here, covalent modifications were shown to be more stable and enable the implementation of well-known, site-directed recognition elements, such as antibodies or synthetic aptamers, through introductions of functional side groups. ${ }^{172,173}$ Resulting changes within the nanotube bond structure, predominantly due to the hybridization from $\mathrm{sp}^{2}$ to $\mathrm{sp}^{3}$ orbitals, however, also risked permanent transformations of the materials optoelectronic properties. ${ }^{115,174}$ Non-covalent modifications, on the contrary, led mainly to the preservation of SWCNT abilities as interactions with the carbon atoms were primarily mediated by weak, attractive forces such as $\pi-\pi$ or $\pi-\mathrm{CH}$ stackings. ${ }^{162,175}$ Common methods, including wrapping of conjugated biopolymers like DNA or chitosan, furthermore enhanced solubilization of the nanotube, thus decreased agglomeration effects and promoted biocompatibility. ${ }^{115,166,176}$ Yet, studies like Fujigaya et al. also accounted for rather low coating stabilities and described the impact and structure of so generated coronas as fairly unpredictable entailing comprehensive screening studies and specificity tests before use. $^{175}$

An example of this can be seen by nanotube modification via nucleic acid polymer wrapping by Kruss et al. which was found to exert increased nIR fluorescence intensity in the presence of specific catecholamines, particularly dopamine and epinephrine, and likewise defines the primary sensor model of this thesis. ${ }^{165,166,177}$ Its general recognition behavior is not fully understood yet, however comprehensive studies and MD simulations made it possible to highlight a dynamic interaction between the hydroxy groups of the dopamine and the phosphate units of the wrapped DNA polymer (figure 7a). ${ }^{123}$ While in contact/close proximity to the phosphate group, the catecholamines are believed to change the local conformation of the DNA subunit and pull it closer to the SWCNT surface leading to a local change of the nanotubes surface potential and thus to a reduction of prevalent quenching/exciton absorption states (figure 7b). 
a

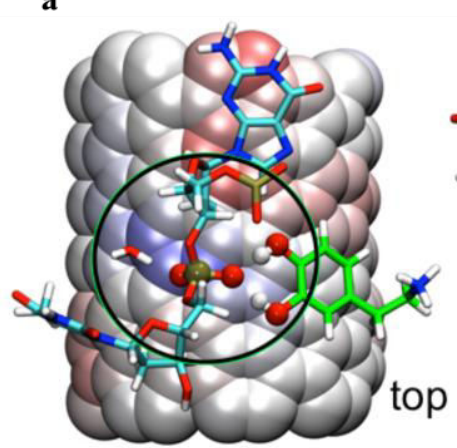

b

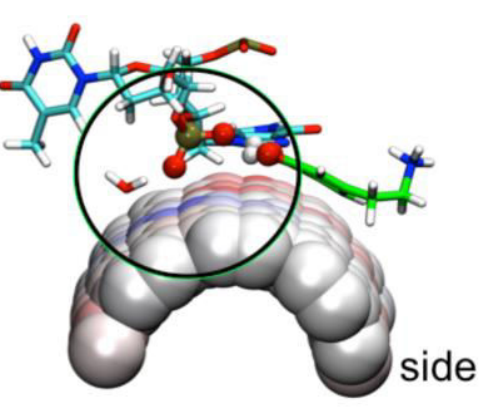

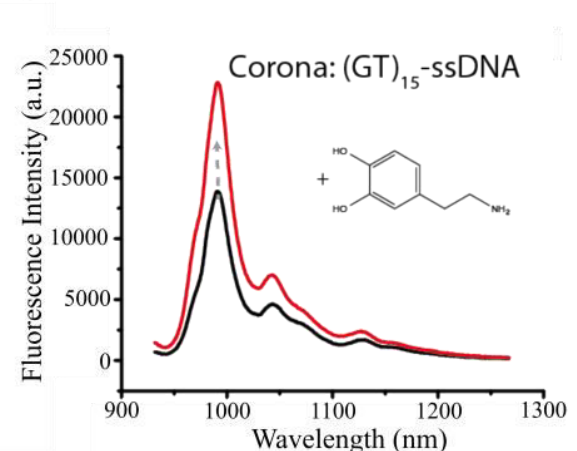

Figure 7: Potential interaction of DNA-wrapped SWCNTs with the target molecule dopamine. a MD simulation of dopamine interaction with a $(\mathrm{GT})_{15^{-}}$DNA-wrapped SWCNT. OH groups of the dopamine (green) are shown as red spheres and pull the $\mathrm{P}_{4}{ }^{-}$unit of the DNA to the nanotube surface resulting in a local, slightly positive potential (blue NT surface) in this area. A supporting water molecule further solvates the associated phosphate group oxygen and thus adds to the potential. b (GT) ${ }_{15}$-DNA wrapped SWCNT spectra without (black) and after addition of dopamine. Due to the aforementioned interaction, the intensity of the characteristic peak increases (images reproduced from ${ }^{123}$ ).

Interestingly, each of the conjugates and DNA-combinations tested in these studies resulted in different signal modulations including intensity signals, binding affinities or chemical detection limits which visualizes, once more, the importance of appropriate surface functionalization for SWCNT-based sensor production. ${ }^{123,165,166}$

\subsubsection{SWCNT based Chemical Imaging}

Due to the diverse nature of nanotube functionalization, the development of functional SWCNT biosensors resembles somewhat the way of a high risk/high reward system. When built correctly, their recognition unit can consist of corona phases capable of transmitting single-molecule interactions while their presence and signal transduction behavior (e.g., fluorescence light) is versatile, biocompatible and enables a spatiotemporal flow of information. ${ }^{123,166,178}$ At the same time, utilization of not well-adjusted sensors, or those that do not match the demand of the experimental setup (e.g., selectivity- or dynamic-wise), could also result to diverging or, worse, misleading conclusions. This holds especially for applications that aim to assess small or fast diminishing amounts of substances, such as chemical imaging processes of cellular in vitro systems, and was characterized in more detail within one of the earlier projects in this thesis (see chapter 3.2). ${ }^{177,179-181}$ Giving a few background information, chemical imaging is usually referred to as procedures that spatiotemporally sense and quantify chemical content and is carried out by measurements of optical properties, in particular, spectra from absorption, emission (fluorescence) or scattering (Raman) events. ${ }^{182,183}$ However, as this definition accounts for wavelength independent metering modes as well, conventional imaging processes like microscopic sequential recordings can be likewise included. Due to this, the typical setup in chemical imaging processes can be diverse (see attached reviews for more detail ${ }^{182,184,185}$ ), yet, most of them require cost-intensive instruments and/or fluorescent labels for chemical targeting, a factor that is continuously discussed to perturb 
functionality within exposed cellular systems. ${ }^{182,184,185}$ Novel nanosensor-based methods, in contrast, might provide a remedy since the sensors characteristic recognition pattern can lead to analyte quantification in the absence of prior cell/system treatments. ${ }^{123,186}$ For this, the experimental design of chemical imaging with nanosensors is usually based on two steps: first, the sensors are homogeneously distributed within the environment of interest, for in vitro cell system, for example, by adsorption onto adhesive 2D-surfaces or into 3D-matrices (figure 8a), and precalibrated by defined exposures of different analyte concentrations. This calibration phase then enables the differentiation of each sensors response function in the presence of a certain, local analyte concentration around them. Thus, by subsequentially adding the biological material, cells or other sought objects to the system, not only the position but also the local amount of generated/depleted substances can be quantified (see also figure $8 \mathrm{~b}$ ). ${ }^{123,124,186,187}$

a

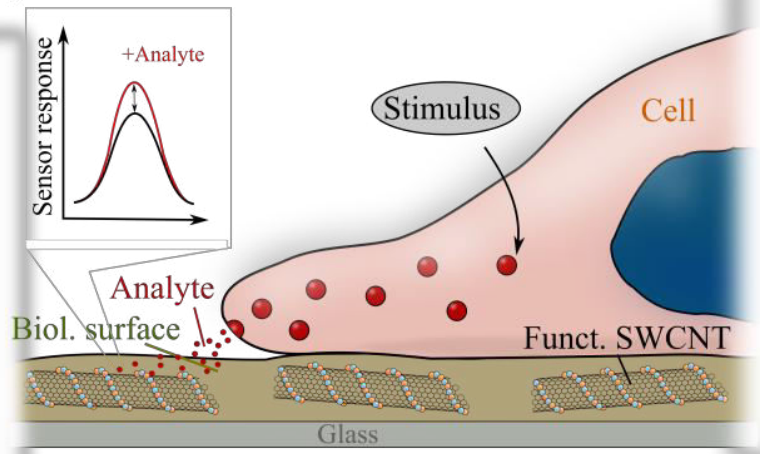

b

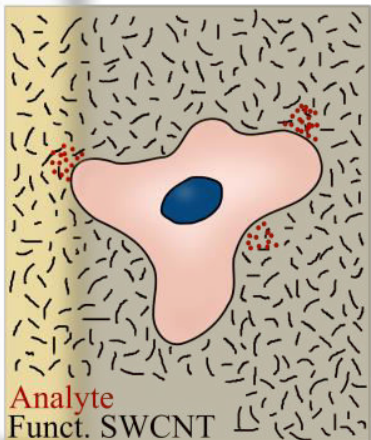

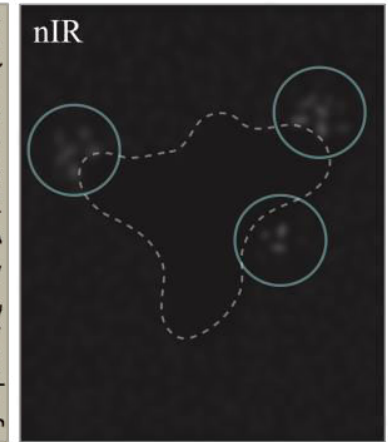

nIR

Figure 8: Principle of chemical imaging with SWCNT sensor patterns exemplified by a schematic single-cell experiment. a Cross section of a chemical imaging setup. Sensors are embedded in a biocompatible surface and/or fixed on glass slides to generate a homogenous sensor surface. With this, while the biological system of choice (cells, artificial processes etc.) generates a timedependent, local change of the corresponding analyte concentration (e.g. a cell releases molecules via exocytosis), surrounding sensors are able to interact and respond to the given molecule variations. This then leads to a spatiotemporal response image (b) that is not only able to localize the position of the release event but is also capable of translating given response values to quantitative, chemical informations (e.g. concentrations, provided the used sensor pattern is pre-calibrated).

Naturally, the outcome and precision of this method is strongly dependant on the dynamics and incidents within each analyzed environment and requires vast knowledge about possible interactions between sensors and the observed material. It can, however, also reward the experimentalist with the quantification of up to know undetectable events. Kruss et al., for example, were able to spatiotemporally detect dopamine release sites from activated pheochromocytoma cells (PC12) by placing the cells on top of their DNAwrapped SWCNT-sensor coated surface whilst Kim et al. built sensor arrays to measure the single molecule efflux of hydrogen peroxide from human umbilical vein endothelial cells (HUVEC) for the first time. ${ }^{123,124}$ 


\section{SUMMARY OF CHAPTER 2.2}

Single-walled carbon nanotubes (SWCNTs) possess remarkable optoelectronic properties that make them a valuable tool for optical biosensor production. Due to their unique $\mathrm{sp}^{2}$ conjugated $\pi$-system, multiple chirality substructures are possible and hold own, individual characteristics. Here, most SWCNTs show a semiconduction behavior and the ability to emit photons in the nIR region $(900-1600$ $\mathrm{nm})$. By using approriate surface modifications, specific interaction cites (corona) can be introduced that may changes the local SWCNT surface potential during analyte interplay and thus generate a response/sensor function at the singlemolecule level. Due to this, SWCNT based nanosensors are highly modifiable, can be made biocompatible and comprise excellent characteristics for spatiotemporal quantification studies such as chemical imaging experiments.

For more, general information about this topic, following reference works have especially proven to provide an useful introduction: ${ }^{116,117,130,131,135,140,150,161}$

\subsection{Stochastic Chemical Kinetics \& Simulation Algorithms}

Admittedly, even though the concept of nanosensor based chemical imaging holds great potential, the development of such systems may require considerable efforts, especially as both, the specificity and the sensors recognition dynamics, have to be precisely adjusted to generate a reliable output. Here, while the former can be tested via screening methods and independent trials, the latter is proven to be a rather hardly ascertainable specification, albeit its vital consequences for the outcome of the corresponding experiment. ${ }^{188-191}$ To illustrate this point, one may think of imaging systems that contain sensors with specific, yet potentially too strong and prolonged interaction/binding effects (high binding affinity) - such systems would interact with first analytes (and for sure sense them) but associated interaction sites would also most likely become oversaturated and fail to recognize subsequent alteration events (see also chapter 3.2, figure 3). Vice versa, systems containing potentially too low affinities would naturally show weak interactions and hardly discernable identification patterns. Thus, producing a functional sensor environment conforms a balancing act that may require continual and time-consuming readjustments depending on the dynamics of the occurring event one sought to determine.

A way to improve this issue was introduced in one of this thesis' projects and involved the theoretical emulation of nanosensor based chemical imaging systems by stochastic simulation algorithms (SSA). ${ }^{177}$ As dynamic sensor properties, such as the association and disassociation constants, can be measured in real-life experiments and implemented into such theoretical model systems, accurate simulations of the sensor's performance might be capable of predicting the efficiency of the functionalized material even before its actu- 
al utilization. ${ }^{192,193}$ As a result, this section shall give a short overview of the general concept of stochastic chemical kinetics and the associated simulation algorithms.

Starting this topic with a standard, kinetical approach, the interaction between a sensor, or respectively one of its binding sites, and a given analyte can be expressed as a limited reaction equation, namely

$$
\mathrm{A}+\mathrm{S} \underset{\mathrm{k}_{\mathrm{off}}}{\stackrel{\mathrm{k}_{\mathrm{on}}}{\rightleftharpoons}} \mathrm{AS}
$$

with A representing the free, diffusive state of the analyte, $\mathrm{S}$ showing the unoccupied phase of a sensor binding site and AS is the analyte-binding site complex that is continuously built or disassembled depending on the reaction rate constants/binding affinities $\mathrm{k}_{\mathrm{on}}$ and $\mathrm{k}_{\text {off. }}{ }^{194,195}$ Following the conventional route, one may further solve the underlying differential equation and expose the prominent, exponential relation between AS and its related rate coefficients, which holds as long as its deterministic ansatz (e.g., by accounting the average behavior of the sensor ensemble) stays justified. ${ }^{194}$ When switching to single molecules regimes now, however, the description of particle interaction goes a different way, predominantly as associated reaction conditions are mainly driven by stochastic, i.e. non-deterministic, forces and thus do not apply to the assumption above anymore. ${ }^{196,197}$ In such cases, especially the likelihood that a reaction per se takes place has to be taken into account and defines the basis for every stochastic chemical kinetic (SCK) theory. ${ }^{197}$

Thus, one may define such a probability $\mathrm{P}$ for an interaction/reaction to occur within a specific, infinitesimal long time interval $[\mathrm{t} ; \mathrm{t}+\mathrm{dt}]$ and a volume $\mathrm{V}$ by its so-called probability density function $\gamma_{\mathrm{r}}$ in such way that

$$
P(\mathbf{N}, V, d t)=\gamma_{r}(\mathbf{N}, V) d t,
$$

with $\mathbf{N}=\left(\mathrm{N}_{1}, \mathrm{~N}_{2}, \ldots, \mathrm{N}_{\mathrm{n}}\right)$ denoting the microstate of the system and $\mathrm{N}_{\mathrm{i}}$ the number of associated reagents generated from the interaction/reaction r. ${ }^{197}$ Here, the structure of $\gamma_{r}(\mathbf{N}, V)$ depends strongly on the type of interactions and has to be adjusted accordingly. Processes that resemble those of uni-molecular reactions ( $\mathrm{X} \rightarrow \operatorname{Product}(\mathrm{s})$ ), for example, were shown to be proportional to $\mathrm{N}_{\mathrm{i}}$ and its reaction rate constant $\mathrm{k}_{\mathrm{i}}$, whereas for bimolecular interactions $(\mathrm{X}+\mathrm{Y} \rightarrow$ Product(s)), also the volume of the system has to be included: ${ }^{197}$

$$
\begin{aligned}
\gamma_{\mathrm{r}}\left(\mathrm{N}_{\mathrm{i}}\right) & =\mathrm{k}_{\mathrm{i}} \cdot \mathrm{N}_{\mathrm{i}} \\
\gamma_{\mathrm{r}}\left(\mathrm{N}_{\mathrm{i}}, \mathrm{N}_{\mathrm{j}}\right) & =\frac{\mathrm{k}_{\mathrm{i}}}{\mathrm{V}} \cdot \mathrm{N}_{\mathrm{i}} \cdot \mathrm{N}_{\mathrm{i}} .
\end{aligned}
$$

Now, in order to predict and describe the actions within a system, normally the microstate $\mathbf{N}(\mathrm{t})$, that is the number of all particles in their state $\mathrm{N}_{\mathrm{i}}(\mathrm{t})$, has to be known for each time 
point $t$ within the (theoretical) experiment. As, however, the stochasticity of the system and its corresponding density functions solely allow for probability statements, one may define a time-dependent probability function, the so-called master equation, instead, that only accounts for the likelihood $\mathrm{P}(\mathbf{N}, \mathrm{t})$ to find the system in a certain state $\mathbf{N}(\mathrm{t}){ }^{197-199}$

$$
\frac{\mathrm{dP}(\mathbf{N}, \mathrm{V}, \mathrm{t})}{\mathrm{dt}}=\sum_{\mathrm{r}} \gamma_{\mathrm{r}}\left(\mathbf{N}-\Delta_{\mathrm{r}}, \mathrm{V}, \mathrm{t}\right) \mathrm{P}\left(\mathbf{N}-\Delta_{\mathrm{r}}, \mathrm{V}, \mathrm{t}\right)-\gamma_{\mathrm{r}}(\mathbf{N}, \mathrm{V}, \mathrm{t}) \mathrm{P}(\mathbf{N}, \mathrm{V}, \mathrm{t})
$$

In general, this differential equation holds the central position in the field of chemical kinetics as it provides a complete description of the stochastic dynamics within a given framework. ${ }^{197}$ Figuratively, it can be read as a loss-gain equation that is comprised of reactions $r$ that either increase the probability to be in the state $\mathbf{N}$, due to other states $\mathbf{N}-$ $\Delta_{\mathrm{r}}$ leading into it (left side of the summation bracket), or decrease the probability due to particle interactions that may point out of it (right side).

Unfortunately finding an analytical solution for this equation becomes most often quite difficult, if not even impossible; and even though suitable approximations improved its overall application to this day, numerical analysis and/or stochastic simulations were usually shown to offer a better prospect of success. ${ }^{200-202}$ In doing so, the common way to apply a numerical analysis to a probability equation is typically defined by consecutive random sampling of the system. ${ }^{200,203}$ By generating a (pseudo)random number that is affiliated with one (or more) stochastic variables of the system, an associated algorithm normally simulates one possible trajectory of the underlying differential equation and consequently decides when or if an interaction, i.e., a change of states, takes place (Monte Carlo simulation). ${ }^{200,204}$ Repeating this process then an appropriate amount of times (each time with the use of a new random number), a deterministic behavior of the system, if available, should come forward (law of large numbers, see also attached source for explanation). ${ }^{200,204}$ Naturally, this simulation step does not have to be limited to one time point or the interactions of one possible particle within the system but can also be applied iteratively, for example, in sequential time steps $[t ; t+d t]$ and to all particles leading to a dynamic, time-dependent simulation of the entire system (Kinetic Monte Carlo algorithm). ${ }^{197,200}$ An example of such a process can be described by the so-called stochastic simulation algorithm (SSA) that was first proposed by Dan Gillespie in 1976 and applied in this thesis to simulate the binding interactions between diffusing analytes and SWCNT-based sensor binding sites. ${ }^{197,205}$ Similar to the aforementioned description, the procedure of such an SSA can be visualized by a repetitive two-tier process: again, using a random number generator, the algorithm first simulates a period $\tau$ at which a next interaction in the system should take place - in our case, for example, a binding or unbinding event of an analyte. Subsequently, it changes the state of the associated 
particles at that time point (bound $\rightarrow$ unbound or vice versa), adjusts the probability values accordingly and simulates the next period at which a change would occur. ${ }^{197}$

Here, the period $\tau$ per se is a stochastic variable and thus dependent on the likelihood $\mathrm{P}(\mathbf{N}, \mathrm{V}, \mathrm{dt})$ of the given interaction. Its mathematical relation can be easily derived using equation (3) in combination with a simple gedankenexperiment: ${ }^{206}$ with the probability $\mathrm{P}(\mathbf{N}, \mathrm{V}, \mathrm{dt})=\gamma_{\mathrm{r}}(\mathbf{N}, \mathrm{V}) \mathrm{dt}$ to find an interaction after a duration of $[\mathrm{t} ; \mathrm{t}+\mathrm{dt}]$, and consequently $\mathrm{P}(\mathrm{N}, \mathrm{V}, \mathrm{dt})=1-\gamma_{\mathrm{r}}(\mathbf{N}, \mathrm{V}) \mathrm{dt}$ to find none, suppose an actual event only takes place after $\mathrm{k}+1$ time steps. The probability of such an occasion then would be

$$
\mathrm{P}(\mathbf{N}, \mathrm{V}, \mathrm{dt})=\left(1-\gamma_{\mathrm{r}}(\mathbf{N}, \mathrm{V})\right)^{\mathrm{k}} \gamma_{\mathrm{r}}(\mathbf{N}, \mathrm{V}) \mathrm{dt}
$$

Now, defining this "waiting time" $\tau=(\mathrm{k}+1) \mathrm{dt}$ and decreasing the size of the time steps $\mathrm{dt}$ by taking the limit $\mathrm{k} \rightarrow \infty$, the probability that an interaction will occur in the next infinitisimal period $[\mathrm{t}+\tau ; \mathrm{t}+\tau+\mathrm{d} \tau]$ would become

$$
\begin{aligned}
P(N, V, d \tau)=\rho(N, v, \tau) d \tau & =\lim _{\mathrm{k} \rightarrow \infty}\left(1-\gamma_{\mathrm{r}}(\mathbf{N}, \mathrm{V}) \frac{\tau}{\mathrm{k}+1}\right)^{\mathrm{k}} \gamma_{\mathrm{r}}(\mathrm{N}, \mathrm{V}) \mathrm{d} \tau \\
& =\mathrm{e}^{-\gamma_{\mathrm{r}}(\mathbf{N}, \mathrm{V}) \tau} \gamma_{\mathrm{r}}(\mathbf{N}, \mathrm{V}) \mathrm{d} \tau
\end{aligned}
$$

As one may see, the probability density $\rho(\mathrm{N}, \mathrm{v}, \tau)$ for this time period depicts an exponentially distributed random variable. ${ }^{206}$ Thus, to implement a random number $q \in$ $(0,1)$, that follows this behavior, or, in other words, determines the probability $q \equiv$ $\mathrm{P}(\mathrm{N}, \mathrm{V}, \tau)=\int \rho(\mathrm{N}, \mathrm{v}, \tau) \mathrm{d} \tau$ for the given time $\tau$, a last, final integration has to be fulfilled and reveals the releation $\tau(\mathbf{N}, \mathrm{V}, \mathrm{q})$ :

$$
\tau=\frac{1}{\gamma_{r}(N, V)} \ln \left(\frac{1}{q}\right) .
$$

With this knowledge in addition to the exact forms of the probability density $\gamma_{r}(\mathbf{N}, V)$ (see again eq. (4) and (5)), the exact iteration steps of an SSA can be described as follows: ${ }^{197}$

1) Initialize the time $t$ and state $\mathbf{N}$ of the system.

2) Evaluate $\gamma_{r}(\mathbf{N}, V)$ by using the informations of state $\mathbf{N}$.

3) Generate a uniformly distributed random number $q \in(0,1)$ and calculate $\tau$ according to equation (10).

4) Effect the reaction by replacing $\mathrm{t} \leftarrow \mathrm{t}+\tau$ and update the state $\mathrm{N} \leftarrow \mathrm{N}+\Delta_{\text {r }}$.

5) Return to step 2.

For more, general information about this topic, especially following reference works are recommended: $194,197,200,203,205,206$ 


\section{Associated Publications}

\subsection{Manuscript 1}

\section{Chromatin Swelling Drives Neutrophil Extracellular Trap Release}

\section{Nature Communications}

Published: 14 September 2018

Elsa Neubert $^{1,2 !}$, Daniel Meyer ${ }^{2,3 !}$, Francesco Rocca ${ }^{3,4}$, Gökhan Günay ${ }^{1,2}$, Anja KwaczalaTessmann $^{1}$, Julia Grandke ${ }^{1}$, Susanne Senger-Sander ${ }^{1}$, Claudia Geisler ${ }^{3,4}$, Alexander Egner $^{3,4}$, Michael P. Schön ${ }^{1,5}$, Luise Erpenbeck ${ }^{1} \&$ Sebastian Kruss $^{2,3}$

${ }^{1}$ Department of Dermatology, Venerology and Allergology, University Medical Center, Göttingen University, Göttingen 37075, Germany

2 Institute of Physical Chemistry, Göttingen University, Göttingen 37077, Germany

${ }^{3}$ Center of Nanoscale Microscopy and Molecular Physiology of the Brain (CNMPB), Göttingen 37073, Germany

${ }^{4}$ Optical Nanoscopy, Laser-Laboratorium Göttingen e.V., Göttingen 37077, Germany

${ }^{5}$ Lower Saxony Institute of Occupational Dermatology, University Medical Center Göttingen and University Osnabrück, Göttingen 37075, Germany

${ }^{\star}$ These authors contributed equally: Elsa Neubert \& Daniel Meyer 


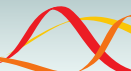

\section{ARTICLE}

\section{DOI: 10.1038/s41467-018-06263-5 OPEN}

\section{Chromatin swelling drives neutrophil extracellular trap release}

Elsa Neubert ${ }^{1,2}$, Daniel Meyer2,3, Francesco Rocca ${ }^{3,4}$, Gökhan Günay ${ }^{1,2}$, Anja Kwaczala-Tessmann', Julia Grandke', Susanne Senger-Sander ${ }^{1}$, Claudia Geisler ${ }^{3,4}$, Alexander Egner ${ }^{3,4}$, Michael P. Schön (1) ${ }^{1,5}$, Luise Erpenbeck (i) ${ }^{1} \&$ Sebastian Kruss (i) ${ }^{2,3}$

Neutrophilic granulocytes are able to release their own DNA as neutrophil extracellular traps (NETs) to capture and eliminate pathogens. DNA expulsion (NETosis) has also been documented for other cells and organisms, thus highlighting the evolutionary conservation of this process. Moreover, dysregulated NETosis has been implicated in many diseases, including cancer and inflammatory disorders. During NETosis, neutrophils undergo dynamic and dramatic alterations of their cellular as well as sub-cellular morphology whose biophysical basis is poorly understood. Here we investigate NETosis in real-time on the single-cell level using fluorescence and atomic force microscopy. Our results show that NETosis is highly organized into three distinct phases with a clear point of no return defined by chromatin status. Entropic chromatin swelling is the major physical driving force that causes cell morphology changes and the rupture of both nuclear envelope and plasma membrane. Through its material properties, chromatin thus directly orchestrates this complex biological process.

\footnotetext{
${ }^{1}$ Department of Dermatology, Venereology and Allergology, University Medical Center, Goettingen University, Göttingen 37075 , Germany. ${ }^{2}$ Institute of Physical Chemistry, Göttingen University, Göttingen 37077, Germany. ${ }^{3}$ Center for Nanoscale Microscopy and Molecular Physiology of the Brain (CNMPB), Göttingen 37073, Germany. ${ }^{4}$ Optical Nanoscopy, Laser-Laboratorium Göttingen e.V., Göttingen 37077, Germany. ${ }^{5}$ Lower Saxony Institute of Occupational Dermatology, University Medical Center Göttingen and University of Osnabrück, Göttingen 37075, Germany. These authors contributed equally: Elsa Neubert, Daniel Meyer. Correspondence and requests for materials should be addressed to L.E. (email: luise.erpenbeck@med.uni-goettingen) or to S.K. (email: skruss@uni-goettingen.de)
} 
$\mathrm{N}$ eutrophilic granulocytes are the most abundant immune cells in humans and essential to defeat invading pathogens $^{1}$. Their mechanisms to target invading microbes include well-known processes such as phagocytosis and generation of reactive oxygen species (ROS). A third defense pathway is the release of neutrophil extracellular traps (NETs) ${ }^{2}$. The formation of NETs (NETosis) can be triggered by organisms such as bacteria or different chemicals and was originally described as an additional form of cell death apart from apoptosis and necrosis ${ }^{3-5}$. NETosis has been reported not only for neutrophils but also other immune cells ${ }^{6,7}$, amoebas ${ }^{8}$ and plant cells ${ }^{9}$ indicating an evolutionary conserved process ${ }^{3}$.

During NETosis, cells can release three-dimensional meshworks (NETs) consisting of chromatin ${ }^{2}$, antimicrobial components including myeloperoxidase $(\mathrm{MPO})^{5}$, neutrophil elastase $(\mathrm{NE})^{10}$, and LL37 of the cathelecidin family ${ }^{11}$. These fibrous networks were initially described as a mechanism to catch and eliminate bacteria, fungi, as well as viral particles ${ }^{2}$. However, it is becoming increasingly clear that the role of NETs in the immune system is far more complex than originally estimated. On the one hand, accumulating data suggests that the immediate role of NETs in immunoprotection against pathogens may be smaller than originally anticipated, as mice that cannot form NETs do not suffer from severe immunosuppression ${ }^{12,13}$. On the other hand, dysregulated or excessive NETosis appears to be implicated in an ever growing number of diseases, including cancer ${ }^{14}$, thrombosis and vascular diseases ${ }^{15-17}$, preeclampsia ${ }^{18}$, chronic inflammatory diseases $^{19}$, and ischemia-reperfusion injury after myocardial infarction 16

Various stimuli such as bacteria, fungi, viruses, platelets, as well as small compounds including lipopolysaccharides (LPS), calcium ionophores (CaI), or phorbol-myristate acetate (PMA) induce NETosis and release of NETs ${ }^{20}$. In many settings, NETosis appears to rely on the adhesion of neutrophils, in particular on the engagement of neutrophilic integrin receptors such as Mac- $1^{21-23}$, in others, adhesion via Mac-1 seems to be dispensable $24-26$. It has also been described that hemodynamic forces can trigger shear-induced NETosis 27 .

While these triggers-biochemical or mechanical-engage diverse pathways, they all converge to a uniform outcome, namely histone modification, chromatin decondensation and NET release ${ }^{28}$. Cells dramatically rearrange their contents (cytoskeleton, organelles, membranes, nucleus) during NETosis; in most scenarios, they eventually die $^{4}$. Chromatin decondensation has been described qualitatively since the discovery of NETs ${ }^{4,29,30}$ and NET formation has been evaluated both in high-throughput approaches, as well as on the single-cell level ${ }^{29-31}$. Yet, the mechanistic basis of these fundamental changes, as well as the underlying dynamic forces remain poorly characterized. Here, we investigate NETosis from a biophysical perspective, particularly looking at the forces and dynamics driving this process, and provide functional links between chromatin dynamics and biochemical behavior. We show that NETosis is organized into well-defined phases orchestrated by entropic swelling of chromatin, which finally ruptures the membrane.

\section{Results}

NETosis is organized into distinct phases. To better understand how the cell's interior is rearranged and how NETs are released we studied human neutrophils in real-time. First, we imaged chromatin and cell membranes of human neutrophils stimulated by $100 \mathrm{nM}$ PMA (Fig. 1a, b, Supplementary Movies 1,2). NETosis was confirmed by co-localization of chromatin and MPO within the expelled NETs (Fig. 1f).
The chromatin-filled area inside the cells followed a characteristic time course (Fig. 1a, b) that consistently allowed the assignment of three distinct phases. As can be seen later this phase classification allows us to distinguish active biological processes from materials driven processes and to identify a point of no return. Cells were stimulated $(t=0)$ and during the first phase $\mathrm{P} 1\left(0<t<t_{1}=\right.$ start of chromatin expansion $)$ the lobular structure of the nucleus was still intact (34 min in Fig. 1a) and the corresponding chromatin area stayed constant. In the second phase P2 $\left(t_{1}<t<t_{3}=\right.$ NET release $)$ chromatin expanded within a few minutes until it reached the cell membrane as a barrier $\left(t_{2}=\right.$ maximal chromatin expansion in Fig. 1a). Simultaneously, the cell rounded up ( $t_{2}$ to $t_{3}$ in Fig. 1a and Supplementary Fig. 1a, b). In the third and final phase (P3, $\left.t>t_{3}\right)$ the cell membrane is ruptured $\left(t_{3}\right)$ and the NET released into the extracellular space. Additionally, released NETs were also visualized by stimulated emission depletion (STED) nanoscopy to reveal the architecture of hydrated NETs below the resolution limit of normal fluorescence microscopy (Supplementary Fig. 2). The observed architecture was in good agreement with previous electron microscopy images of NETs ${ }^{2}$.

The above-mentioned phase classification was applied to multiple cells ( $n=139$ cells) from five different donors (Fig. 1c, Supplementary Fig. 3a-c, Supplementary Movies 3-6). Although the onset time points for the different phases of all individual cells followed a broad distribution (Fig. 1c), average onset values for all five donors were remarkably reproducible, indicating low interindividual variability for the three distinct phases under standardized conditions (Supplementary Fig. 3c). Decondensation of chromatin started at $t_{1}=56 \pm 4 \mathrm{~min}$ (standard error of the mean, SEM) and reached a maximum at $t_{2}=82 \mathrm{~min}( \pm 3 \mathrm{~min})$. After $t_{3}=116 \mathrm{~min}( \pm 4 \mathrm{~min})$ the cytoplasmic membrane ruptured and the NETs were released. In summary, P1, as well as P2, require around $60 \mathrm{~min}$ in our experimental setup.

The three distinct phases of chromatin decondensation were not only elicited by PMA but also by LPS (Fig. 1d) or calcium ionophores (Fig. 1e), albeit with different onset times, particularly with respect to P1 (Supplementary Fig. 3d, Supplementary Movie 7), which is most likely an expression of the distinct signaling pathways engaged by different stimuli ${ }^{28}$.

To understand biophysical events during NETosis in detail, we analyzed chromatin shape with special regard to the most relevant internal and external boundaries (i.e., nuclear envelope and cell membrane). In P1, cells first adhered to the surface, flattened and showed filopodia activity (Supplementary Fig. 4a, b, Supplementary Movies 1, 2, 8). During P2, the cell retracted its cell body as shown by CLSM (Fig. 1a, Supplementary Fig. 4a, b) and time-resolved reflection interference contrast microscopy (RICM) (Supplementary Fig. 4c, d and Supplementary Movies 9-11), the cells rounded up and their height increased as demonstrated by both three-dimensional CLSM stacks (side view, Fig. 2a) and atomic force microscopy (AFM) (Fig. 2b).

Interestingly, NETotic cells reached approximately the same height as non-stimulated round cells (Fig. 2b), suggesting that chromatin expansion/decondensation is a swelling process and the swelling pressure caused the energetically most favorable spherical shape.

To address the question of how chromatin exits the nucleus, we labeled lamin B1, a constitutive component of the nuclear envelope that surrounded all individual lobuli of the nucleus (Fig. 2c, Supplementary Fig. 5). Over time, those lobuli merged and became less distinctive, indicating profound changes in the separation between chromatin. Around the onset of P2 $\left(t_{1}\right.$; i.e., the start of chromatin expansion), the lamin B1 layer tore on at least one site of the nucleus. Often, several rupture events of this layer were discernable (arrows Fig. 2c, see also comparison 
a

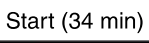

$t_{1}(60 \mathrm{~min})$

$t_{2}(80 \mathrm{~min})$
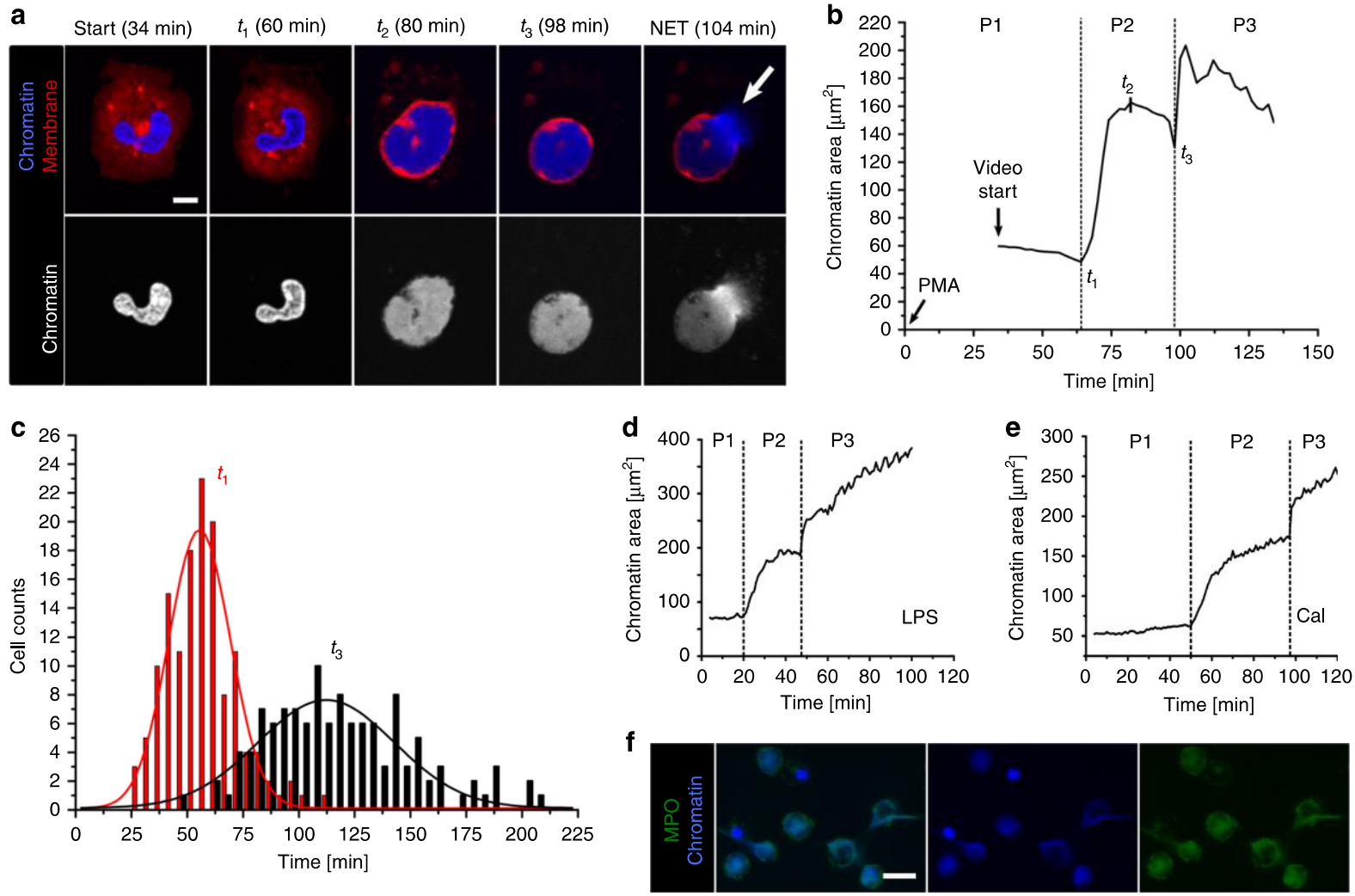

Fig. 1 Phases of NETosis. a Morphological changes of chromatin (blue) and cell membrane (red) during NETosis of human neutrophils (stimulated with $100 \mathrm{nM}$ PMA) imaged by live-cell confocal laser scanning microscopy (CLSM). The lobular nucleus loses its shape and chromatin decondenses until it fills the entire cell. Finally, the cell rounds up and releases the NET (white arrow). Scale bar $=5 \mu \mathrm{m}$. b Corresponding chromatin area of a NETotic neutrophil (a) as a function of time reveals three distinct phases. P1: Activation, lobulated nucleus. P2: Decondensation/expansion of chromatin within the cell ( $t_{1}=$ start of chromatin expansion, $t_{2}=$ maximal chromatin expansion within the cell); cell rounding. P3: Rupture of the cell membrane (arrow a) and NET release ( $t_{3}$ $=$ NET release). $\mathbf{c}$ Histogram of onset times of the different phases. $n=139$ cells. $N=5$ donors. Lines represent Gaussian distribution function fits. $\mathbf{d}$ Time course of chromatin area for stimulation with LPS (Lipopolysaccharide, from Pseudomonas aeruginosa, $25 \mu \mathrm{g} \mathrm{ml}{ }^{-1}$ ). e Time course of chromatin area for stimulation with calcium ionophore $(4 \mu \mathrm{M})$. c-e data acquired with live-cell wide field fluorescence microscopy. $\mathbf{f}$ Colocalization of decondensed neutrophil chromatin (blue) and myeloperoxidase (green). Fixed cells imaged by wide field fluorescence microscopy. Scale bar $=20 \mu \mathrm{m}$

of chromatin area at $t_{1}$ in Supplementary Fig. 5). Similar fluorescent labeling methods have been used by others to quantify nuclear envelope rupture events ${ }^{32}$. Previous publications have described the modification of nuclear lamins by phosphorylation as an early event, which would affect rigidity and could facilitate the here-described breakage of the nuclear envelope $^{33}$. It should be noted that the breakage of the nuclear envelope appears to be a distinct process from the previously described dissolution of the nuclear envelope, which is a hallmark of late stages of NETosis ${ }^{13,33}$.

In line with these previously published observations, we could show that the nuclear envelope further decomposed during P2 and P3 and lamin B1 was found distributed throughout the cytoplasm (Fig. 2c, P2/3). Subsequently, nuclear envelope breakdown allowed further expansion and swelling of chromatin within the cell (Fig. 2d). Consequently, the temporal correlation between $t_{1}$ and the rupturing of the nuclear envelope indicates that chromatin swelling is the physical driving force of this event.

The dissection of NETosis into distinct phases allowed us to identify and distinguish active (biological/biochemical) and passive (material properties) events. In the next step, we linked the phase classification to biochemical processes.
Active and passive mechanisms during NETosis. The initial steps of NETosis are thought to rely on several enzymes, with the exact progression depending greatly on the activator used to initiate NET formation ${ }^{28}$. In most scenarios, NETosis depends on the activity of typical neutrophil enzymes such as neutrophil lactase (NE) and myeloperoxidase (MPO) ${ }^{34}$, as well as histone citrullination by the enzyme peptidyl-arginine deiminase 4 (PAD4). However, it is unclear at which time point during NETosis signaling and activity by these players are essential, and whether they initiate or maintain the process. If they were required in $\mathrm{P} 1$, and later phases were governed by passive mechanisms such as swelling of chromatin, then P2 and P3 should not depend on an active cellular energy supply. Indeed, ATP levels of (PMA) activated neutrophils quickly decreased in P1 by up to $70 \%$, particularly within the first $30 \mathrm{~min}$, indicating energy-dependent processes (Fig. 3a). In contrast, ATP levels then remained constant throughout P2 on a low level (Fig. 3a). To corroborate the hypothesis that energy supply is not necessary for $\mathrm{P} 2$, the main energy source in neutrophils, glycolysis ${ }^{35,36}$, was cut off by inhibiting glucose metabolism with 2-Deoxy-D-glucose

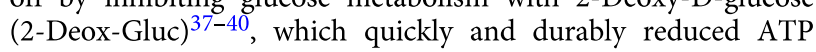
levels of neutrophils as early as 15 min in PMA-stimulated cells 
a

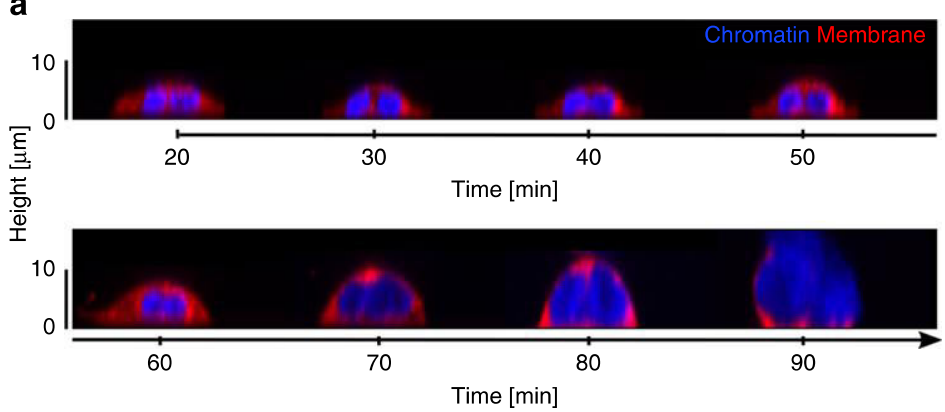

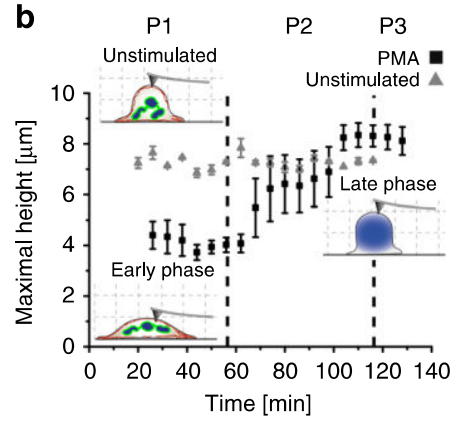

P1

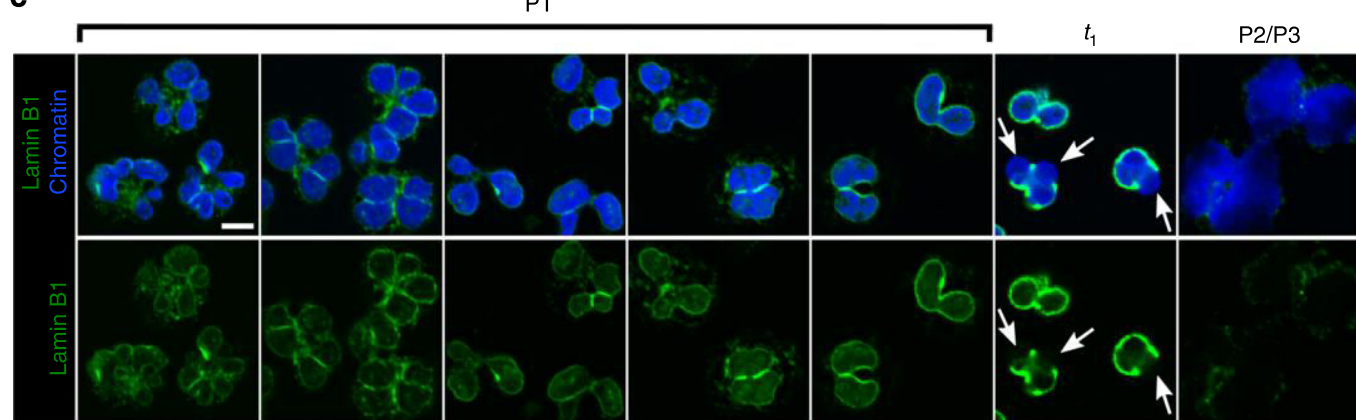

d

P1

P2

P3

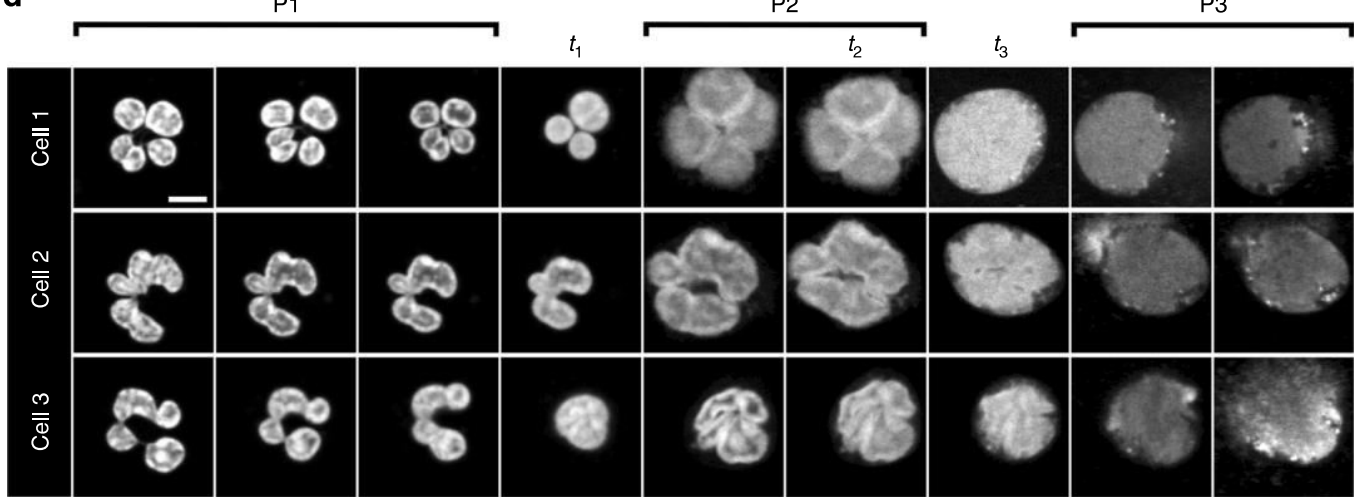

Fig. 2 Chromatin swelling drives morphological changes. a Live-cell CLSM side view of a neutrophil during NETosis. Chromatin (blue) decondenses/ expands, reaches the membrane (red) and the cell rounds up until the membrane ruptures. z-stack depth: $1 \mu \mathrm{m}$. $\mathbf{b}$ Cell height as measured by atomic force microscopy (AFM) on life neutrophils. PMA stimulated cells adhere and flatten (compared to the control cells that stay more or less round) and then round up $(>8 \mu \mathrm{m})$ in P2. $n=3$. Mean \pm SEM. c Characteristic distribution of lamin B1 (green) in the three phases, CSLM images of fixed cells. Lamin B1 first surrounds single lobuli of the nucleus/chromatin (blue). When chromatin starts to expand corresponding to the start of P2 (around $t_{1}$ ), the lamin B1 layer/ nuclear envelope ruptures on at least on one side of the nucleus. During P2 and P3 lamin B1 further decomposes. White arrows indicate rupture sites of the lamin B1 layer. Scale bar $=5 \mu \mathrm{m}$. d The original shape of the nucleus remains recognizable during the expansion process, particularly in the first part of P2 $\left(t_{1}\right.$ to $\left.t_{2}\right)$, indicating isomorphic chromatin swelling and not directional transport (Supplementary Movies 13-15). In P1 the nucleus has a lobulated structure, which is maintained (self-similarity) during P2. Finally, the membrane is reached and, for a short period of time, this barrier prevents further expansion until it burst. Scale bar $=5 \mu \mathrm{m}$. Live-cell CSLM images

and within $60 \mathrm{~min}$ in unstimulated neutrophils (Supplementary Fig. 6 b, c). Additionally, sodium azide $\left(\mathrm{NaN}_{3}\right)$ was used in this setup as a general inhibitor of metabolic function and, specifically, of metalloproteases ${ }^{41-43}$, and MPO was inhibited with 4aminobenzoic acid hydrazide $(4-\mathrm{ABAH})^{44,45}$. We verified the function of these enzymatic inhibitors by showing that $\mathrm{NaN}_{3}$ inhibits ROS production in neutrophils immediately after addition for at least $30 \mathrm{~min}$ (Supplementary Fig. 6a and 9c) and that 4-ABAH inhibited purified MPO within $1 \mathrm{~min}$ and stable within 15 min after PMA activation (Supplementary Fig. 6d). Thus, 4ABAH directly interferes with PMA-induced NET formation ${ }^{5}$. None of the here-used inhibitors had any measurable effect on
NET-production of naïve neutrophils (Fig. 3b), nor did any of them show significant toxicity (Supplementary Fig. 7a). As expected, however, effects of these inhibitors were not exclusive to NET-formation, as 4-ABAH and 2-Deox-Gluc clearly decreased the uptake of FITC-labeled E. coli particles, although $\mathrm{NaN}_{3}$ showed no effect in this setup (Supplementary Fig. 7b).

When added directly after stimulation all inhibitors significantly reduced NET formation (Fig. 3b). This effect successively decreased when inhibitors were added at later time points. Exposure to $\mathrm{NaN}_{3}$ after $>60$ min and to 2-Deox-Gluc or 4-ABAH after $>75 \mathrm{~min}$ no longer affected the number of decondensed nuclei. This result again implies that P1 depends on energy supply 
a

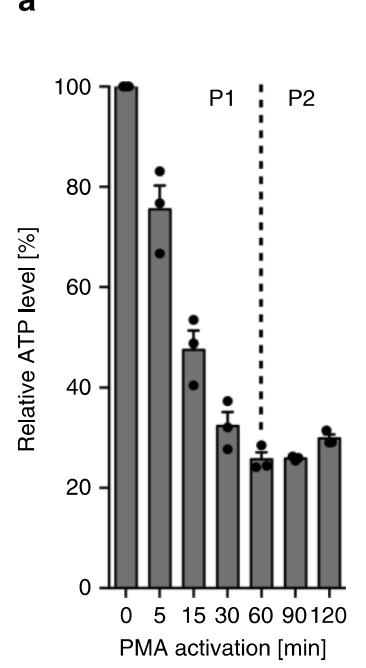

b

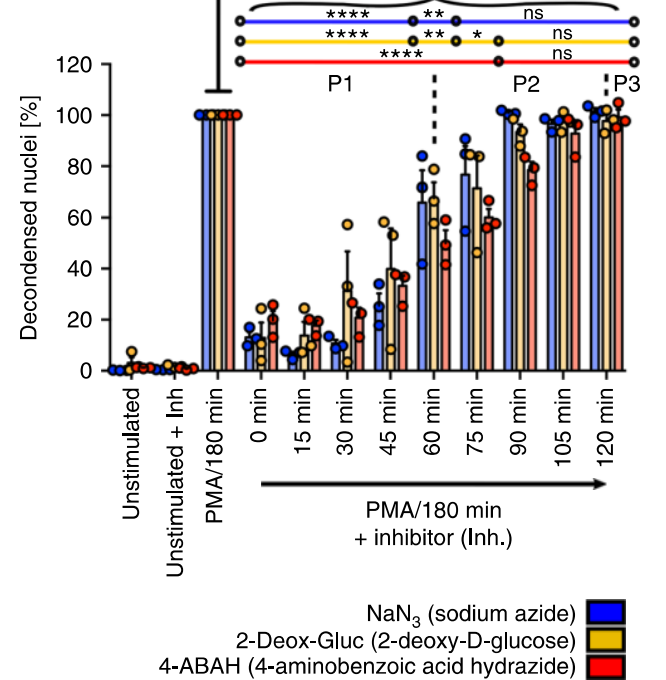

c

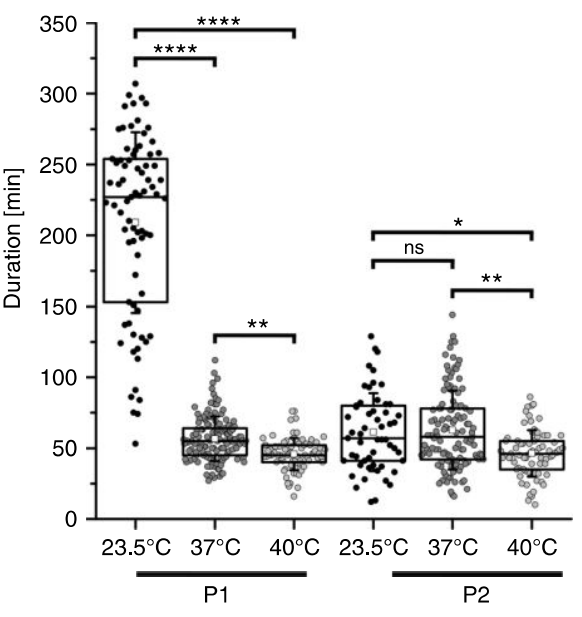

Fig. 3 Active and passive processes during NETosis. a ATP levels in stimulated neutrophils decrease during P1 and reach a plateau in P2. Inhibition of glucose metabolism further reduces ATP levels (Supplementary Fig. 6c). $N=3$. Mean \pm SEM. b Metabolic inhibitors (sodium azide/3 mM, 2-deoxy-Dglucose $/ 5 \mathrm{mM}, 4$-aminobenzoic acid hydrazide/100 $\mu \mathrm{M}$ ) influence NET formation determined as relative number of decondensed nuclei after 180 min compared to activation with PMA only. All inhibitors decrease NET formation when added in P1, while P2 is not or only slightly affected, indicating a point of no return. $N=3$ donors. Statistics: two-way ANOVA (Bonferroni's multiple comparisons test; ${ }^{\star} p<0.05 ;{ }^{\star \star} p<0.01 ;{ }^{\star \star \star \star} p<0.0001 ; n s=$ not significant). Mean \pm SEM. c Phase duration at different temperatures $\left(23.5,37,40^{\circ} \mathrm{C}\right)$. P1 is significantly prolonged at lower temperatures, whereas $\mathrm{P} 2$ displays no or marginal temperature dependence. $N=3\left(23.5,40^{\circ} \mathrm{C}\right) . N=5\left(37^{\circ} \mathrm{C}\right)$. Statistics: Kruskal-Wallis test (Dunn's multiple comparisons test; ${ }^{*} p<0.05$; ${ }^{\star \star} p<0.01 ;{ }^{\star \star \star \star} p<0.0001 ; \mathrm{ns}=$ not significant). Life-cell imaging. Boxplots display the 25 th and 75 th percentile and the horizontal line the median. Hollow squares represent the mean and whiskers the SD

and enzymatic activity, while P2 and P3 do not. So far, NETosis has been generally considered as an active process that requires the aforementioned enzymes. Here, we show that this generalization does not hold true for the complete mechanism.

Another hallmark of enzymatic activity is temperaturedependence. We chose to show the impact of temperature variations on the impact of NETosis as a complementary, inhibitor-free approach to investigate the importance on enzymatic activity. We quantified the duration of the different phases of NETosis at physiological core temperature $\left(37^{\circ} \mathrm{C}\right)$, hypothermia $\left(23.5^{\circ} \mathrm{C}\right)$ and hyperthermia/fever $\left(40^{\circ} \mathrm{C}\right)$. Higher temperatures significantly accelerated $\mathrm{P} 1$ whereas $\mathrm{P} 2$ showed no or only a slight temperature dependence (Fig. 3c, Supplementary Movie 12), indicating high enzyme activity in P1. If one assumes Arrheniuslike kinetics $\left(k \sim \exp \left(-\frac{E_{\mathrm{a}}}{k_{\mathrm{B}} T}\right)\right)$, the 4.14-fold shortened duration of P1 $\left(227.5 \mathrm{~min}\right.$ at $23.5^{\circ} \mathrm{C}$ vs. $55.0 \mathrm{~min}$ at $\left.37^{\circ} \mathrm{C}\right)$ corresponds to an activation energy of around $80 \mathrm{~kJ} \mathrm{~mol}^{-1}$, which falls into the range expected for enzyme-catalyzed reactions ${ }^{46}$, and again corroborates our hypothesis of a switch from biochemically driven processes to behavior governed by material properties. P3 was not evaluated in the context of temperature-dependency as it is not possible to determine an end-point of $\mathrm{P} 3$ after the release of the NET.

Additionally, this result indicates an enzyme activity independent diffusive process in P2 since one expects lower temperature dependence for diffusion. In the first part of P2 (Fig. 1b, 2d, Supplementary Fig. 3a, Supplementary Movies 13-15) the chromatin area $A$ increased linearly with time $t$ and can be interpreted as a $2 \mathrm{D}$ diffusion process $\left(A \approx\left\langle x^{2}\right\rangle=4 D t\right)$. The corresponding effective diffusion constant $D$ of $0.0108 \mu \mathrm{m}^{2} \mathrm{~s}^{-1}$ at $37^{\circ} \mathrm{C}$ (Supplementary Fig. 8a) is roughly in agreement with the diffusion of a $2 \times 10^{9}$ DNA sequence of $\left(D \approx 0.002 \mu \mathrm{m}^{2} \mathrm{~s}^{-1}\right.$, $\left.\mathrm{T}=37^{\circ} \mathrm{C}\right)^{47,48}$.
Entropic chromatin swelling drives morphological changes. At the beginning of NETosis (P1 in our classification), histones are modified chemically (decrease of positive charge) by enzymes such as PAD4 or NE, which reduces the counterforces that hold the negatively charged DNA/chromatin together ${ }^{10,49}$. A condensed nucleus is under considerable entropic pressure as the radius of gyration of the human genome (length around $2 \mathrm{~m}$ ) is approximately $150-200 \mu \mathrm{m}^{50,51}$. Once the counterforces are no longer high enough to balance the entropic pressure, chromatin begins to swell. This time-point corresponds to $t_{1}$ (onset of P2) and marks a point of no return.

Another line of evidence pointing to entropic pressure as a relevant factor stems from the observation that small neutrophils rupture faster than larger ones after chromatin filled the whole cell lumen (Fig. 4a). As all cells contain the same amount of chromatin, entropic pressure exerted on the cell membrane is higher if they are smaller. This should lead to earlier rupturing of the membrane. Likewise, large intact neutrophils accumulate during the experiments because smaller ones rupture and release NETs first (Fig. 4b).

To analyze whether the swelling pressure generated during P2 determines if and when the membrane ruptures, we calculated the pressure exerted by chromatin and compared it to the rupture delay time $\left(t_{2}\right.$ to $\left.t_{3}\right)$. For that purpose we modified a NavierStokes equation-based theory that describes pressure as a function of time $t$ (see Methods ${ }^{52}$ ):

$$
p(t)=\frac{\eta(R(t))}{l_{p}^{2}(R(t))} \frac{\mathrm{d} R(t)}{\mathrm{d} t} R(t)
$$

Here $\eta$ is the viscosity of the chromatin (liquid), $R(t)$ the effective radius of the chromatin area and $l_{p}$ the chromatin mesh size. STED nanoscopy images of chromatin just before the cells 

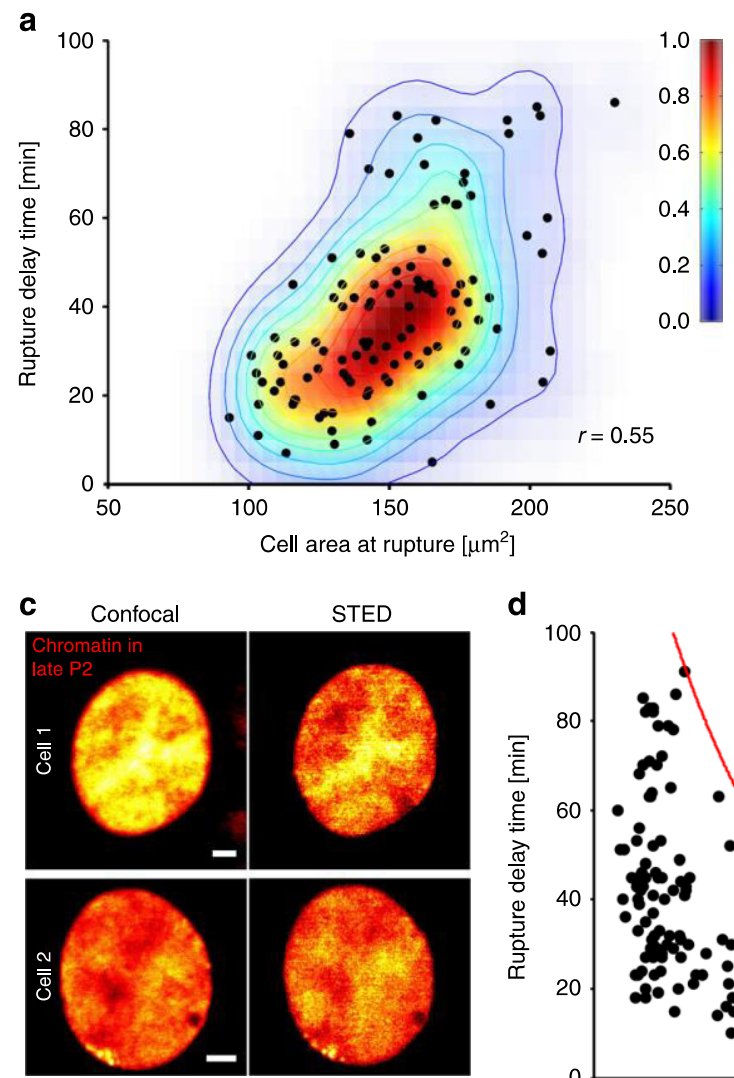
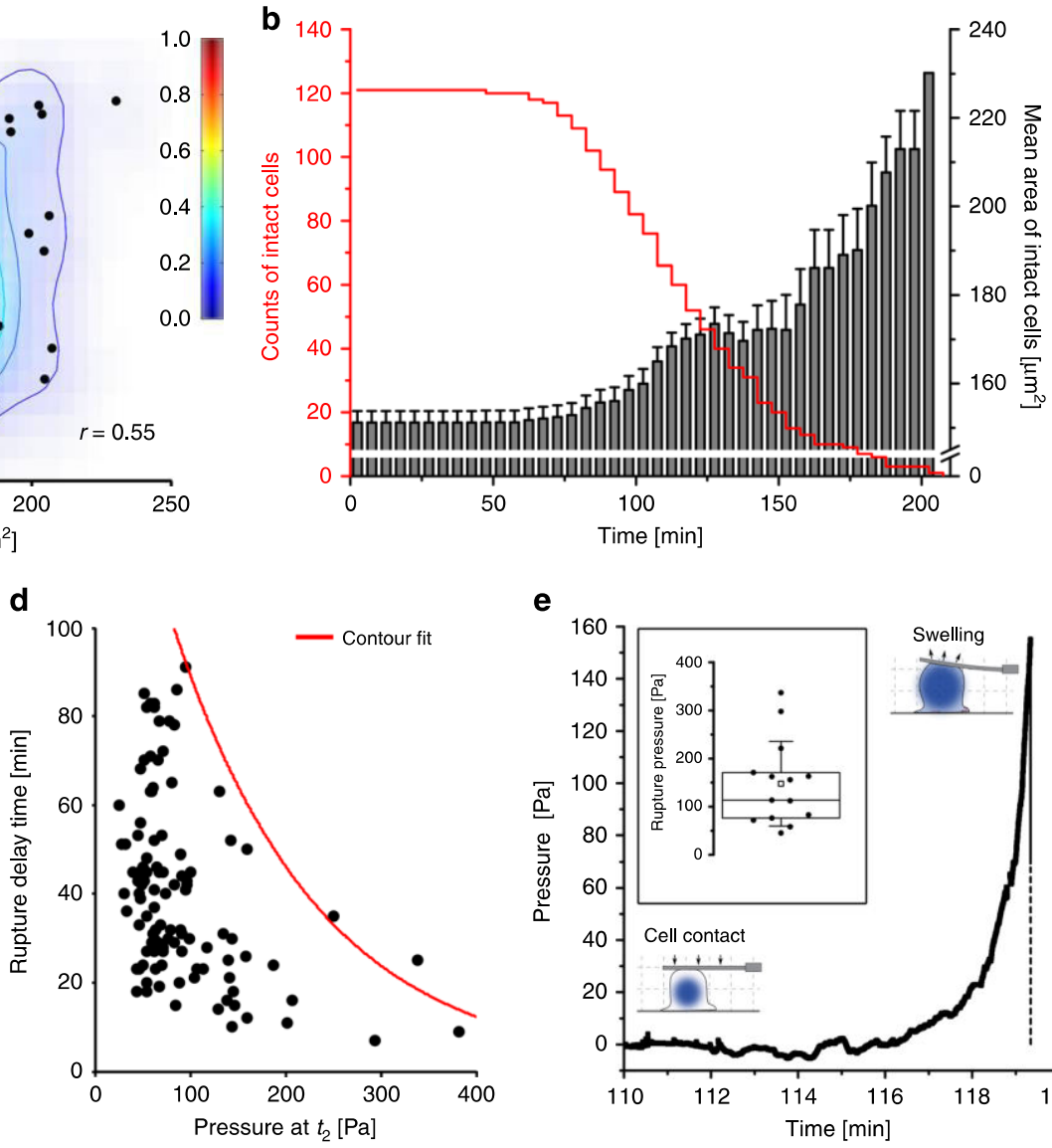

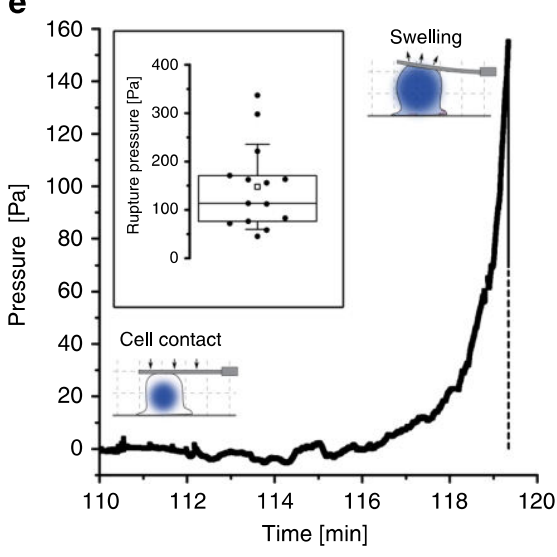

Fig. 4 Entropic swelling of chromatin causes membrane rupture. a Correlation between cell area at $t_{3}$ (chromatin area at $t_{3} \approx$ total cell area at $t_{3}$ ) and time span until membrane rupture occurs following maximal chromatin expansion ( $t_{3}-t_{2}$, rupture delay time). Larger cells rupture later than smaller cells. $n=$ 112 cells (only cells of population 1 included, see Supplementary Fig. 3a, b). Fit lines show normalized 2D-probability density function calculated by kernel density estimation (KDE). $N=5$ donors. $\mathbf{b}$ Average cell area (at $t_{3}$ ) of remaining/intact cells. The area of intact cells increases from $\approx 151$ to $\approx 230 \mu m^{2}$ with time, indicating earlier rupture of small cells. $n=121$ cells. $N=5$ donors. Mean \pm SEM. c Live-cell confocal (left row) and STED (right row) images of chromatin (SiR-DNA) of two neutrophils undergoing NETosis (late P2) show almost uniform distribution of chromatin suggesting a mesh size smaller than the resolution of the microscope $(\approx 120 \mathrm{~nm}(x y)$ and $\approx 150 \mathrm{~nm}(z)$, Supplementary Fig. $8 b)$. Scale $=2 \mu \mathrm{m}$. $\mathbf{d}$ Time delay until rupture as a function of the calculated pressure $p$ at $t_{2} . n=112$ cells (only cells of population 1 included, see Supplementary Fig. 3a, b). $N=5$ donors. The contour fit is generated by fitting the maximal data points to an exponential decay curve. e Neutrophils undergoing NETosis exert an increasing pressure on a fixed AFM cantilever until they rupture (end point of measurement). Inset: $N=5(n=14$ cells). Boxplots display the 25th and 75 th percentile and the horizontal line the median. Hollow squares represent the mean and whiskers the SD

ruptured showed no fine structure indicating that the mesh size is below the resolution of this microscope (about $\approx 120 \mathrm{~nm}$ in $\mathrm{xy}$ direction and $\approx 150 \mathrm{~nm}$ in $\mathrm{z}$-direction) (Fig. $4 \mathrm{c}$, Supplementary Fig. $8 \mathrm{~b}, \mathrm{c}$ ). Therefore, we assumed that the whole genome (around $2 \mathrm{~m} \mathrm{DNA}$ ) is evenly arranged inside the cell and estimated that $l_{p}$ to be around $20 \mathrm{~nm}$. Cells exposed to higher pressure ruptured faster than their smaller counterparts (Fig. 4d). The calculated pressure values are in a similar range as pressure values known from osmotic lysis experiments of lipid vesicles ${ }^{53}$. To find out if these calculated pressure values were actually exerted by the cell we measured these forces directly with AFM (Fig. 4e, Supplementary Fig. 8d). A cantilever was positioned in direct contact with the cell and deflected by the rounding cell. This is a known approach to measure rounding forces e.g. during mitosis ${ }^{54}$. The pressure exerted by the cell increased with time and lifted the cantilever until the cell finally ruptured (end point of measurement). The measured pressure values between 100-200 $\mathrm{Pa}$ are in the same range as the calculated pressure values from Fig. $4 \mathrm{~d}$. Together with the control experiments that show that energy supply (Fig. 3) and the actin cytoskeleton (Fig. 5, below) are not crucial for P2 this result further supports chromatin swelling as the major driving force in $\mathrm{P} 2$.

In conclusion, our results show that P1 of NETosis is a biologically active process governed by signaling and enzymatic reactions. In contrast, P2 is mainly passive, based on entropic chromatin swelling and cannot be stopped once it has started. Therefore, the onset of $\mathrm{P} 2\left(t=t_{1}\right)$ represents a point of no return.

Impact of cytoskeletal rearrangements on NETosis. In addition to the unexpected importance of chromatin dynamics reported this far, the cytoskeleton may also contribute to shape shifts and DNA expulsion. While cytoskeletal components appear to rearrange and F-actin may become degraded during NETosis ${ }^{4,34}$, these processes remain poorly understood. Figure $5 \mathrm{a}$ shows the Factin distribution (labeled with SiR-actin) at different time points after PMA-stimulation. During the first 30 min F-actin became more prominent as expected for stimulation with the protein-kinase $\mathrm{C}$ activator PMA, which has multiple effects on 
a

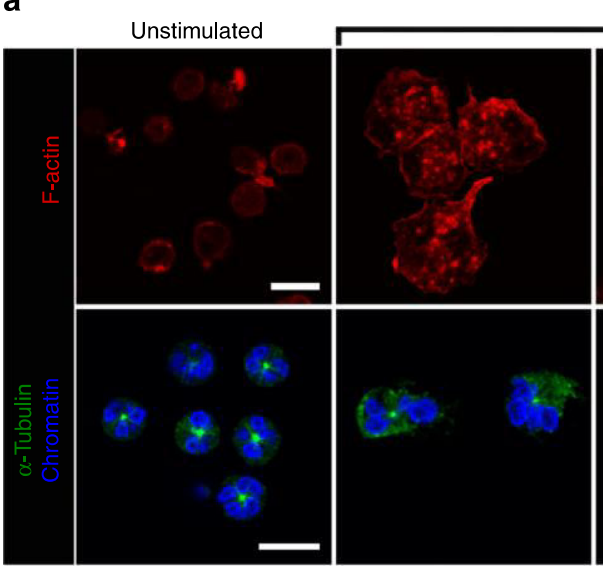

P1
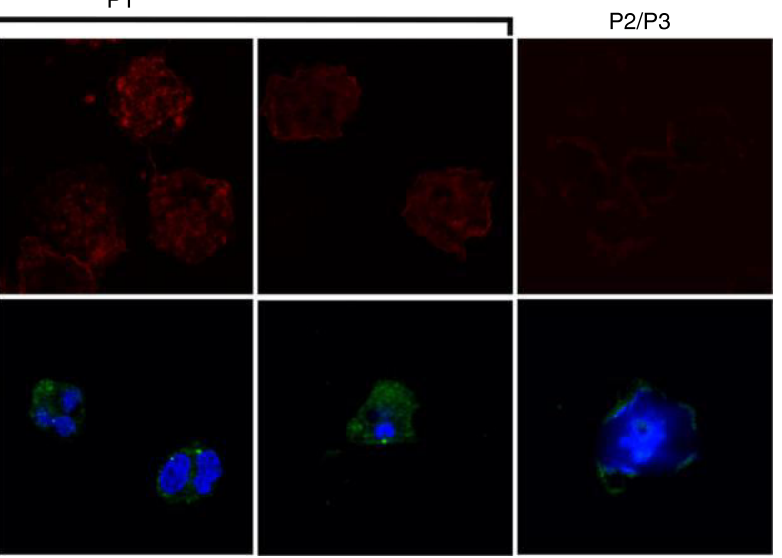

b

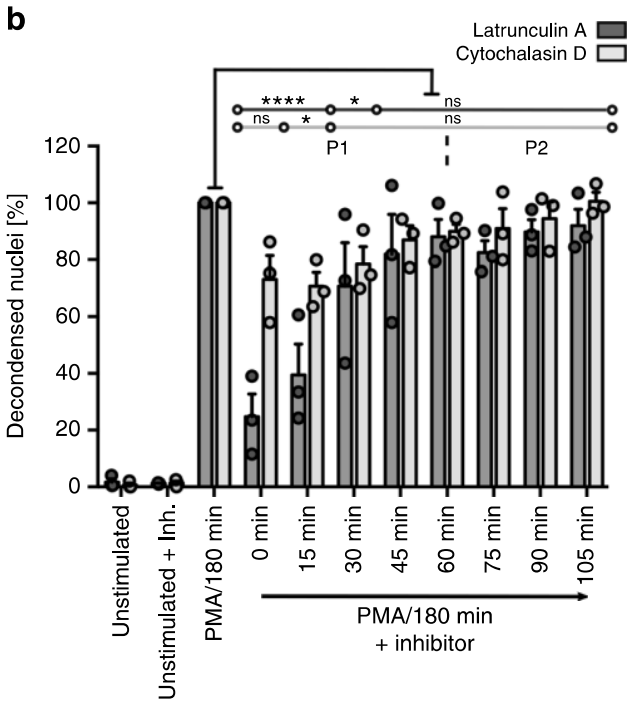

d

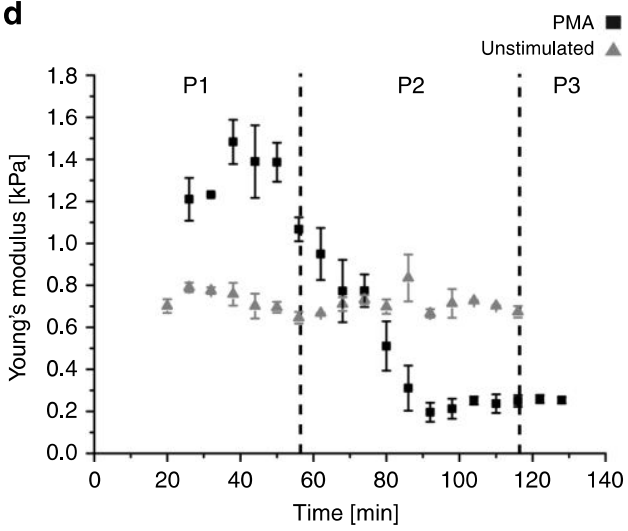

neutrophils, among them the induction of chemotaxis ${ }^{55}$. Then, it diminished and completely disassembled after 90-180 min (see quantification in Supplementary Fig. 9a).

As recently described, a-tubulin is initially organized in filaments originating from the microtubule organization center (MTOC; unstimulated cells) ${ }^{33}$. One could speculate that these MTOCs are involved in active transport of chromatin. However, during phase I after PMA-stimulation, a-tubulin filaments disappeared and were rearranged into dot-like structures,
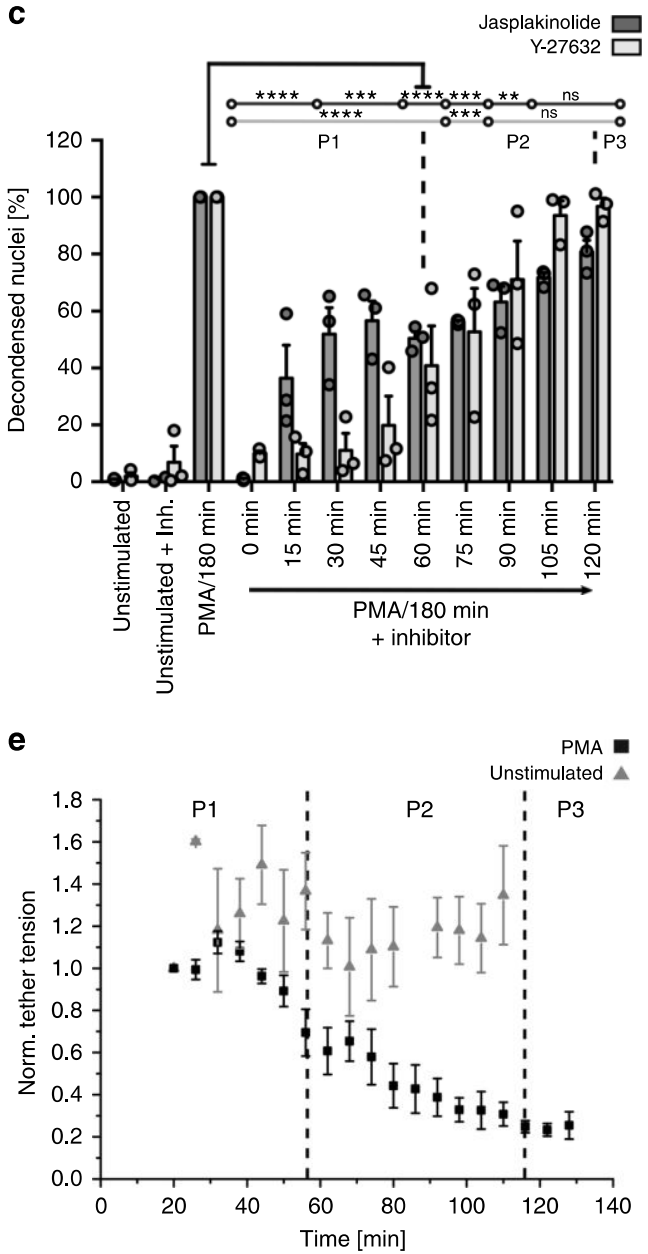

reminiscent of mitotic centrosomes. These were visible until the beginning of chromatin decondensation, namely until the beginning of phase 2 (Fig. 5a). As we did not observe these filaments any more during phase 2 , it is unlikely that active chromatin transport along these centrosomes like structures plays a role during the expansion of chromatin.

Additionally, we sought to determine the role of the cytoskeleton through specific inhibition: Jasplakinolide $(10 \mu \mathrm{M}$, actin stabilization/induction of actin polymerization), 
Fig. 5 Rearrangement of the cytoskeleton and evolution of mechanical properties. a At the beginning of NETosis F-actin (red) is laterally enriched and localizes in the lamellipodia. $\alpha$-Tubulin filaments (green) are arranged originating from the microtubule organizing center (MTOC) in unstimulated cells. Within the next hours (during P1) cytoskeletal components disintegrate. Remaining F-actin accumulates at the cell margin and $\alpha$-tubulin is first rearranged in centrosome-like structures which disappear at the beginning of P2. CLSM images of fixed cells. Activation $=\mathrm{PMA}(100 \mathrm{nM})$. Blue $=$ chromatin. Scale $=$ $10 \mu \mathrm{m}$. b, c Inhibition of NET formation with the F-actin polymerization inhibitors Cytochalasin D (100 nM) and Latrunculin A (1 $\mu$ M), F-actin-stabilizing drug Jasplakinolide $(10 \mu \mathrm{M})$ and the ROCK-inhibitor Y-27632 (19.2 $\mu \mathrm{M})$ significantly reduces the formation of NETs (measured as \%-relative number of decondensed nuclei after 180 min compared to activation with PMA only) in P1, while P2 depends less or not on F-actin stabilization and ROCK-inhibition. Statistics: two-way ANOVA (Bonferroni's multiple comparisons test; ${ }^{\star} p<0.05$; ${ }^{\star \star} p<0.01 ;{ }^{\star \star \star} p<0.001 ;{ }^{\star \star \star \star} p<0.0001$; ns $=$ not significant). $N=3$ donors. Mean \pm SEM. $\mathbf{d}$ Normalized tether tension of life neutrophils (measured with AFM) decreases over the entire time course (raw data: $>0.35 \mathrm{mN} m$ to $<0.07$ $\mathrm{mN} \mathrm{m}$ ) of PMA-activated NETosis indicating a loss of cytoskeletal stability. Values of control cells remain stable. $N=3$. Mean \pm SEM. e Cell stiffness (Young's modulus) of life neutrophils decreases from $>1.5 \mathrm{kPa}$ to $<0.3 \mathrm{kPa}$ after stimulation with PMA whereas the stiffness of control cells remains constant. $N=3$. Mean \pm SEM

Cytochalasin D (100 nM, inhibition of actin polymerization), Latrunculin A $(1 \mu \mathrm{M}$, inhibition of actin polymerization) and Docetaxel (100 nM, inhibition of tubulin depolymerization) were added at different time points after stimulation (with $100 \mathrm{nM}$, PMA). While Docetaxel exerted no (Supplementary Fig. 9b), Cytochalasin D and Latrunculin A showed a significant reduction of NETosis when added up to $15 \mathrm{~min}$ after activation (Fig. 5b). Jasplakinolide inhibited NETosis completely when added from the beginning. The latter effect decreased as NETosis advanced (Fig. 5c). Based on this finding we concluded that reorganization of the cytoskeleton is not only a consequence of NETosis, but also a requirement during the active phase $(\mathrm{P} 1$; Fig. 5b, c). Additionally, we manipulated cell motility by inhibiting the rho-associated coiled-coil-containing protein kinase 1 and 2 (ROCK 1/2) signaling pathway using Y-27632 (19.2 $\mu \mathrm{M})$. Again, we found a strong influence on P1 (Fig. 5c). These results are in good agreement with our results on enzyme activity (Fig. 3b). While it has been shown that cytoskeletal inhibitors may influence the NADPH oxidase ${ }^{56}$, we could rule out that these off-target effects were causing the reduction in NETosis, as Jasplakinolide inhibited ROS formation, while Latrunculin A increased it and the Y-27632 and Cytochalasin D had no or only a slight effect on ROS production (Supplementary Fig. 9c). Thus, while effects on ROS production were very heterogeneous, the effect of cytoskeletal inhibition on NET production showed the same pattern for all used inhibitors (Fig. 5b, c). Therefore, we can rule out that the observed effects on NETosis are exclusively mediated by an influence on the NADPH oxidase. None of our cytoskeletal inhibitors produced any significant cytotoxic effect on neutrophils (Supplementary Fig. 7a).

In conclusion, NETosis requires an intact and functional actin cytoskeleton at the beginning of $\mathrm{P} 1$ and the reorganization of F-actin is essential to proceed to P2 and P3. Rearrangement of the microtubule apparatus appears to be a marker of the activation of biochemical pathways required for NETosis (namely the activation of cyclin-dependent kinases 6 and 4$)^{33}$ and an influence of certain microtubule inhibitors has been postulated by isolated publications ${ }^{57}$. However, in our setup the microtubule apparatus itself appears to be dispensable for NET formation. Taken together, both the actin cytoskeleton of the neutrophils, as well as the microtubule apparatus become dysfunctional as NETosis progresses. Therefore, it is unlikely that the cytoskeleton plays an active role during the final steps of NETosis. Dissolution of these major components, which normally stabilize the cell, likely impairs the cell's mechanical properties, thus helping the final cell membrane rupture.

To test this hypothesis, the cells' mechanical properties were measured by atomic force microscopy (AFM) (Fig. 5d, e, Supplementary Fig. 10, Supplementary Movie 16). Indeed, cells became markedly softer during NETosis, particularly in P2, as evidenced by a decrease of the Young's modulus from $E=1.5$ to $0.3 \mathrm{kPa}$ (Fig. $5 \mathrm{~d}$ ).

Additionally, retraction curves of individual membrane tethers were analyzed to distinguish the cell's bulk mechanical properties from the mechanical properties of the membrane alone. The membrane tension decreased by $77 \%\left(0.35\right.$ to $\left.0.07 \mathrm{mN} \mathrm{m}^{-1}\right)$ (Fig. 5e). Although alterations of the plasma membrane are likely to occur in an inflammasome-dependent manner during NETosis ${ }^{58}$, such a dramatic decrease cannot be explained by changes of membrane composition only, but must be caused by the disassembly of the actin cortex beneath the membrane ${ }^{59,60}$. The membrane tension $T$ in late $\mathrm{P} 2$ corresponds to a membrane pressure $p \approx \frac{2}{R} T \approx 20 \mathrm{~Pa}$ (Young-Laplace equation, radius $R \approx \mu \mathrm{m}$ ). The calculated swelling pressure (Fig. $4 \mathrm{~d}$ ) is in the same range indicating that chromatin swelling is sufficient to break the membrane.

Location of membrane rupture. To test the hypothesis that the chromatin swelling pressure determines the membrane breaking point, we correlated swelling speed and anisotropy with the position of the rupture point. Even though swelling itself should be isotropic, the position of the nucleus/lobuli inside the cell varied with respect to the cell boundary. We first looked at chromatin decondensation and calculated the average swelling speed during $t_{1}<t<t_{2}$ (represented by a velocity field, Fig. 6a, Supplementary Fig. 11). Interestingly, in cells in which the nucleus was closer to one side of the cell membrane, the rupture point was usually close to areas of low chromatin movement, e.g., in close proximity to the cell membrane (cell 1-3). In these cases, there was less space for chromatin to expand (higher remaining entropic pressure). If (in the minority of cells) the nucleus was centered in the cell the rupture direction was random (cell 4).

Moreover, cells typically ruptured at the side that experienced the last membrane retraction close to the long axis of the cells before they rounded up (Fig. 6b).

In order to quantify these observations, a rupture point axis was determined by connecting the rupture point $\mathrm{A}$ with the center of mass of the respective cell $(M)$. Then, the cells' outlines were fitted with an ellipse and the cell membrane shrinking velocities of both cell sides (A and $\mathrm{B}$ ) were analyzed along the rupture axis $\left(v_{\mathrm{A}}\right.$ and $\left.v_{\mathrm{B}}\right)$ during the last minutes before rupture (30 $\mathrm{min} \pm 16 \mathrm{~min}$ ) (Fig. 6c). Cells ruptured at the side that experienced significantly more movement after the rounding process started in late P2 $\left(t>t_{2}\right)$ (Fig. 6d). Most rupture events occurred close to the previously long axis of the (elliptic) cell (Fig. 6e), thus allowing the prediction of the NET release location.

This analysis of membrane morphology shows that cells become circular before the membrane ruptures on the last moving side close to the previous long axis. Additionally, the position of the nucleus with respect to the membrane determines 
a

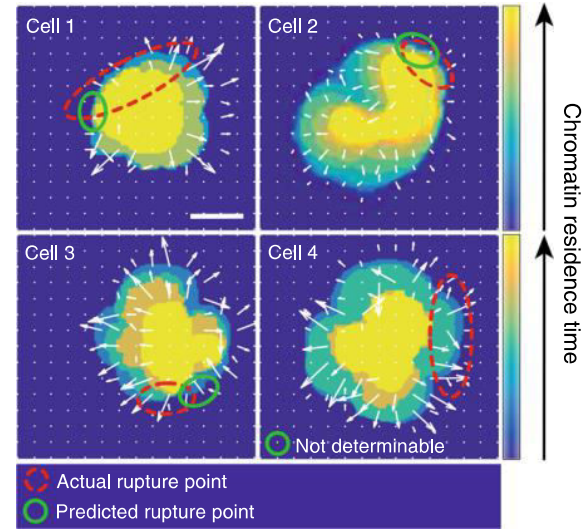

d

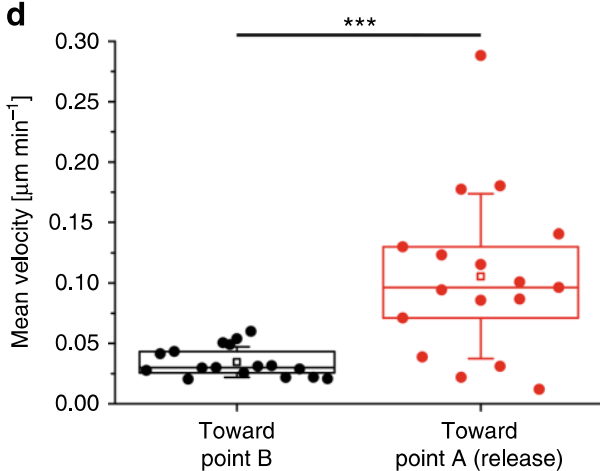

b

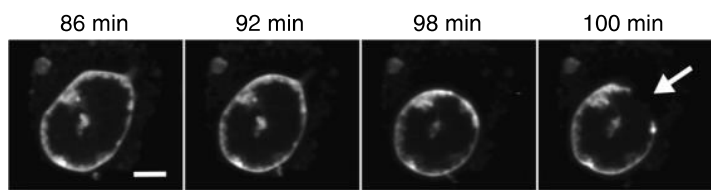

C . Cell rupture axis determination

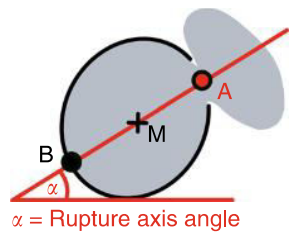

2. Cell rounding analysis

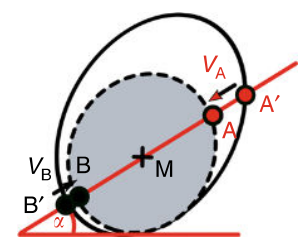

e

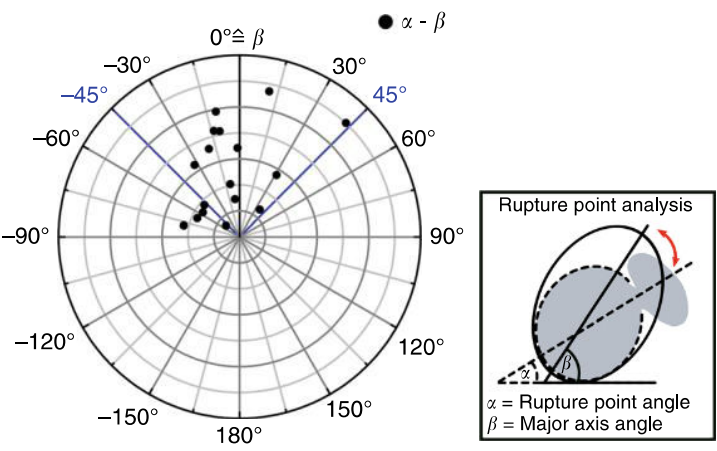

Fig. 6 Predetermination of the membrane rupture point. a Velocity plots of chromatin swelling. Changes to darker colors indicate faster movement (shorter residence time) of chromatin. The actual rupture point (red circle) often correlates with areas of slow movement (predicted rupture point, green circle). b Live-cell CLSM images of the cell membrane (PKH26 staining) directly before NET release. The cell rounds up and ruptures when maximum circularity is reached $(t=98 \mathrm{~min})$. Scale bar $=5 \mu \mathrm{m}$. c Schematic of the rupture point analysis. Rupture points were analyzed by (1) fitting an ellipse to the cell before it became round and determining the rupture axis between the rupture point $A$ and the center of mass $M$ and (2) determining the retraction speed ( $v_{A}$ and $V_{B}$ ) on both sides of the (previously elliptic) cell (see Methods). d Shrinking velocity of the two opposing cell poles (A and B). The neutrophil retracts its membrane with a significantly higher velocity at the future rupture site (A). $n=17 . N=4$ independent experiments. Statistics: Mann-Whitney test, twotailed $\left({ }^{\star \star \star} p<0.001\right)$. Boxplots display the 25 th and 75 th percentile and the horizontal line the median. Hollow squares represent the mean and whiskers the SD. e Angle plot shows that the membrane ruptures in proximity of the major axis. $\alpha=$ rupture point angle. $\beta=$ major axis angle. $n=17$. $N=4$ independent experiments

on which side of the cell entropic chromatin pressure becomes higher.

An additional aspect that has not been addressed in detail so far is influence of adhesion in NETosis. We used reflection interference contrast microscopy (RICM) (Supplementary Fig. 4c, d, Supplementary Movies 9-11) to image the interface between cell and substrate and address this question ${ }^{61,62}$. Neutrophils quickly and strongly adhered and left membrane behind, while rounding up. To further test the impact of adhesion we quantified NETosis on differently coated surfaces (Supplementary Fig. 12). Interestingly, on surfaces passivated with poly(L-lysine)-graftpoly(ethylene glycol) (PLL-g-PEG) neutrophils still performed NETosis even though they could not properly adhere (Supplementary Movie 17). This result does not rule out an important role of adhesion in determining the threshold for NETosis especially for different (weaker) activators. However, it shows that once the cell initiated NETosis, additional adhesive cues were not important anymore. Nevertheless, the influence of external factors for the initiation and execution of NETosis, including adhesive cues and surface characteristics like surface stiffness, for example, certainly warrant further investigation in the future.

\section{Discussion}

Over the last years, much effort has been put into unraveling the signaling cascades and enzymatic players that are indispensable for NETosis. Here, we provide a comprehensive and unique picture of the complex biophysical aspects that govern the different phases of NETosis using an interdisciplinary approach and innovative imaging methods. We conclude that NETosis is a highly organized process with a first phase (P1) that is governed by biochemical modifications including histone citrullination and phosphorylation of lamins ${ }^{33}$ that prepare the cell for later mechanical changes. We show that a point of no return exists after which active processes such as enzymatic activities become secondary and the cells behavior is determined by the characteristics of chromatin (Fig. 7, Supplementary Fig. 13). Morphological changes, as well as rupture/burst of nuclear envelope and cell membrane are driven by entropic swelling of chromatin. At this point, pharmaceutical inhibition of NETosis is no longer possible. These findings may also prove important for other biological processes, such as cell division or other forms of cell death $^{54,63}$. Indeed, it has been shown very recently that broad overlaps exist between NETosis and mitosis from a biochemical 


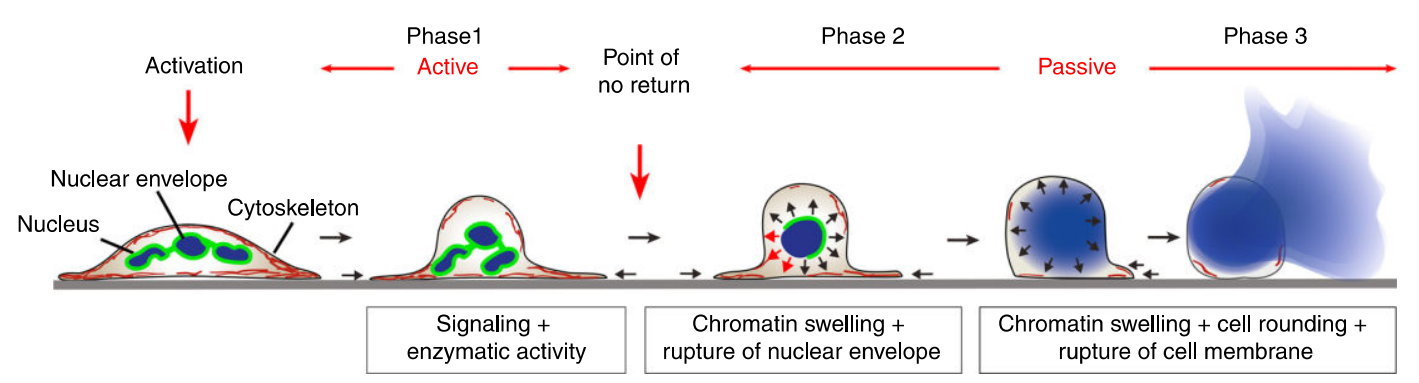

Fig. 7 Biophysical model of NET release NETosis can be divided into three distinct phases (according to chromatin status) that are separated by a point of no return. The major physical driving force for morphological changes and NET release after phase 1 is entropic swelling of chromatin

point of view. It will be worthwhile studying these similarities from a biophysical perspective ${ }^{33}$.

Ultimately, cells appear to be more than biochemical factories and complex processes such as NETosis can therefore be driven not only by biochemical signaling but also material properties.

Chromatin is traditionally viewed as a nuclear entity that mainly regulates gene expression. Here, we show that chromatin is much more than a template for information processing but is able to actively conduct biological processes.

\section{Methods}

Isolation of human neutrophils. Neutrophils were isolated from human venous blood of healthy donors. The study was approved by the ethics committee of the university medical center goettingen (chairman Prof. Dr. med. Jürger Brockmöller) and fully informed consent of all donors obtained after clearing the possible consequences of the study. The isolation was performed according to previous published standard protocols. ${ }^{1}$

In short, fresh blood was collected with S-Monovettes $\mathrm{KE} 7.5 \mathrm{ml}$ (Sarstedt). Blood was gently layered in a 1:1 ratio on top of Histopaque 1119 (Sigma-Aldrich) and centrifuged at $1100 \times g$ for $21 \mathrm{~min}$. Then, the transparent third and pink fourth layer containing the white blood cells were collected and mixed with HBSS (without $\mathrm{Ca}^{2+} / \mathrm{Mg}^{2+}$, Thermo Fisher Scientific). Cells were pelleted by centrifugation for $10 \mathrm{~min}$ at $400 \times \mathrm{g}$. After discarding the supernatant, the pellet was resuspended in HBSS without $\mathrm{Ca}^{2+} / \mathrm{Mg}^{2+}$ and layered on top of a phosphate buffered percoll (GE Healthcare) gradient with the concentrations 85, 80, 75, 70, and $65 \%$ and centrifuged at $1100 \times g$ for $22 \mathrm{~min}$. The accumulated neutrophils were received by collecting half of the $70 \%$, full $75 \%$ and half of the $80 \%$ layer and washed with HBSS. The remaining cell pellet was resuspended in $1 \mathrm{ml}$ HBSS. Cells were counted and suspended at the required concentration for the following procedures with RPMI 1640 (Lonza) containing $10 \mathrm{mM}$ HEPES (Roth) and 0.5\% human serum albumin (HSA) (Sigma-Aldrich). Experiments with lipopolysaccharides (LPS from Pseudomonas aeruginosa serotype 10.22, strain: ATCC 27316, Sigma-Aldrich) or calcium ionophores (CaI, Sigma-Aldrich) were carried out without addition of $0.5 \%$ human serum albumin. Cellular identity was confirmed by a cytospin assay (Cytospin 2 Zentrifuge, Shanson) followed by Diff Quick staining (Medion Diagnostics). Cell purity was $>95 \%$ of isolated cells (without erythrocytes)

Live cell imaging (fluorescence microscopy). Fresh isolated human neutrophils were seeded $\left(4-5 \times 10^{5}\right.$ cells per $\left.\mathrm{ml}\right)$ on ibidi treat flow chambers ( $\mu$-Slide I 0.8 Luer ibidi Treat, ibidi $\mathrm{GmbH})$ for $30 \mathrm{~min}\left(37^{\circ} \mathrm{C}, 5 \% \mathrm{CO}_{2}\right)$ and stained with $1.62 \mu \mathrm{M}$ Hoechst 33342 (Sigma-aldrich) for $15 \mathrm{~min}\left(37^{\circ} \mathrm{C}, 5 \% \mathrm{CO}_{2}\right)$. For membrane staining, cells were stained before seeding with $2 \mu \mathrm{M}$ PKH26 (PKH26-kit, Sigmaaldrich) following the companies' instructions. Cells were activated for NETosis with $100 \mathrm{nM}$ Phorbol-12-myristate-13-acetate (PMA, Sigma-aldrich), $4 \mu \mathrm{M}$ CaI or $25 \mu \mathrm{g} \mathrm{ml}^{-1}$ LPS. Live cell imaging was performed at $23.5,37$ or $40^{\circ} \mathrm{C}$ (ibidi heating system, ibidi $\mathrm{GmbH}$ ) for $3-5 \mathrm{~h}$ with minimized light exposure. Bright field microscopy movies were obtained $\times 16$ magnified (EC Plan-Neofluar Ph1/4403319901-000, Zeiss) using the camera CoolSNAP ES (Photometrics) and the microscope Axiovert 200 (software: Metamorph 6.3r2., Molecular Devices, Zeiss) with a frame rate of one picture per minute in the blue channel (Filter set 02 shift free/ 488002-9901-000, Zeiss). For CLSM images an Olympus IX83 inverted microscope (software: Olympus Fluoview Ver.4.2, Olympus) was used and the movies were recorded $\times 60$ magnified (UPlanSApo 1.35 oil, Olympus). Hoechst fluorescence was detected at $405 \mathrm{~nm}$ and PKH26 fluorescence at $561 \mathrm{~nm}$. All 2D-movies were obtained with a frame-rate of one picture per $2 \mathrm{~min}$ and the 3D-movies with one picture per ten minutes and a $z$-stack depth of $1 \mu \mathrm{m}$ per slice. All videos and pictures were further processed with ImageJ (v. 1.46r ad 1.50c4; National Institutes of Health) and MATLAB (v. R2008a/R2014a; The MathWorks, Inc.) as described in the section statistics and data analysis.
Inhibitor experiments. Fresh isolated human neutrophils (10,000 per well) were seeded in 96-glassbottom-well-plates (In vitro scientific) and activated for NETosis with PMA, final concentration $100 \mathrm{nM}$. Subsequently, the function of cytoskeletal components was inhibited with Cytochalasin D (100 nM, Abcam), Latrunculin A (1 $\mu \mathrm{M}$, Sigma-Aldrich), Docetaxel (100 nM, Abcam), Jasplakinolide (10 $\mu \mathrm{M}$, Enzo) or Y-27632 (19.2 $\mu \mathrm{M}, \mathrm{Abcam})$ and enzyme activity inhibited with 2-deoxy-Dglycosis (2- Deox-Gluc, $5 \mathrm{mM}$, Sigma-aldrich), sodium azide $\left(\mathrm{NaN}_{3}, 3 \mathrm{mM}\right.$, Merck) or 4-Aminobenzoic acid hydrazide (ABAH, $100 \mu \mathrm{M}$, Cayman) at defined time points $(0 \mathrm{~min}, 15 \mathrm{~min}, 30 \mathrm{~min}, 45 \mathrm{~min}, 60 \mathrm{~min}, 75 \mathrm{~min}, 90 \mathrm{~min}, 105 \mathrm{~min}$, and 120 $\mathrm{min}$ ) after activation. All experiments were performed in triplicates. To stop NET formation, cells were fixed with 2\% PFA final concentration (Roth) after $3 \mathrm{~h}$ incubation $\left(37^{\circ} \mathrm{C}, 5 \% \mathrm{CO}_{2}\right)$ and stored over night at $4{ }^{\circ} \mathrm{C}$. The fixed probes were washed 10 min with $1 \times$ PBS (Lonza) and chromatin stained with Hoechst at room temperature. After staining, cells were washed with PBS and imaged with the microscope Axiovert 200 ( $\times 16$ magnification, Zeiss; software: Metamorph 6.3r2, Molecular Devices) and a CoolSNAP ES camera (Photometrics) in the blue channel (Filter set49 DAPI shift free, 488049-9901-000, Zeiss). For each well in total 5-6 images of different regions were collected. For all experiments, the amount of decondensed nuclei, as well as the total cell count was quantified (blinded) with ImageJ. Percentages of decondensed nuclei/NETs were calculated relative to the amount of decondensed nuclei/NETs after stimulation of cells with PMA for $3 \mathrm{~h}$

ATP measurements. Fresh isolated human neutrophils (10,000 per well in RPMI (10 mM HEPES, $0.5 \%$ HSA without phenolred)) were seeded in white 96-wellplates (Greiner bio-one) and activated with PMA in a final concentration of 100 $\mathrm{nM}$ for defined time points ( $5 \mathrm{~min}, 15 \mathrm{~min}, 30 \mathrm{~min}, 60 \mathrm{~min}, 90 \mathrm{~min}$, and $120 \mathrm{~min}$ ) After incubation, CellTiter-Glo ${ }^{\circledR}$ Reagent (Promega) was added in a 1:1 ratio and the ATP amount was determined following the company instructions. In short, the mixture was shaken for $2 \mathrm{~min}$ to induce cell lysis and incubated for $10 \mathrm{~min}$ at room temperature to stabilize the luminescence signal. Subsequently, luminescence was measured (GLOMAX ${ }^{\circledR} 96$ Microplate Luminometer, Software: GLOMAX 1.9.3, Turner BioSystems) and the ATP levels were calculated relatively to the ATP amount of unstimulated cells incubated for $120 \mathrm{~min}$.

Staining procedures. Fresh isolated human neutrophils (200,000 per well) were seeded on pretreated ( $99 \%$ alcohol) glass cover slips (\#1.5) in 24-well plates (Greiner bio-one) and NET formation induced with $100 \mathrm{nM}$ PMA. Cells were then fixed at different time points after NET formation with 2\% PFA and stored in PBS over night The following staining procedure was carried out based on previous published protocols ${ }^{64}$. Briefly, cover slips were gently removed from the 24 -well plate and layered upside down on the washing solution (1xPBS). Cells were then permeabilized with a $0.1 \%$ TritonX (Merck) containing solution for $10 \mathrm{~min}$ at $4{ }^{\circ} \mathrm{C}$, washed and blocked with $5 \%$ fetal calf serum (FCS, Merck) or 3\% BSA (Lamin B1 staining). Subsequently cells were stained with monoclonal anti-human MPO (IgG, mouse, 1:500) (ab25989, Abcam), monoclonal anti-human $\alpha$-Tubulin (IgG, rabbit, 1:50) (\#2125, Cell Signaling Technology) or polyclonal anti-human lamin B1 (IgG, rabbit, 1:1000) (ab16048, Abcam) as primary antibodies over night $\left(4^{\circ} \mathrm{C}\right)$ and visualized with polyclonal antimouse Alexa488 (IgG, goat, 1:1000) (\#4408, Cell Signaling Technology) or polyclonal anti-rabbit Alexa488 (IgG, goat, 1:500) (A-11034, ThermoFisher Scientific) as secondary antibodies. In case of staining with SiR-dyes, cells were not permeabilized but directly stained after washing with SiR-Actin (SC001, Spirochrome AG/Tebu-bio) or SiR-DNA (SC007, Spirochrome AG/Tebu-bio) at $3 \mu \mathrm{M}$. Then, chromatin was stained with Hoechst if applicable and cover slips were mounted with Faramount Mounting Medium (Dako Agilent Technologies) on microscopy slide. After complete drying and fixation with nail polish, samples were imaged with 40x magnification (PlanNeofluar $40 \times / 1.30$ oil Iris/4440456-0000-000, Zeiss) in a fluorescence microscope (AxioImager M1, Software: AxioVision Rel.4.7, Zeiss) or $\times 60$ magnified with confocal laser scanning microscopy (Olympus IX83 inverted microscope, software: Olympus Fluoview v.4.2)

3D-STED microscopy. In conventional optical microscopy, the resolution is limited by diffraction to about half the wavelength of light $(\lambda / 2 \approx 300 \mathrm{~nm})$ and 
about the wavelength $(\lambda \approx 600 \mathrm{~nm})$ in the lateral and axial direction, respectively. To resolve smaller features samples were stained with SiR-DNA as described in the staining procedure section and were imaged with a super-resolution STED (Stimulated Emission Depletion) microscope ${ }^{65}$. For live cell imaging, cells (10,000 pe well) were seeded for $30 \mathrm{~min}$ in 10-well CELLview ${ }^{\mathrm{TM}}$ glass slides (Greiner Bio-one) in RPMI (10 mM HEPES, $0.5 \%$ FCS without phenolred) and activated with $100 \mathrm{nM}$ PMA and stained with $1 \mu \mathrm{M}$ SiR-DNA. In a canonical STED microscope, the diffraction-limited excitation spot is superimposed with a red-shifted donut-shaped laser beam (STED beam) featuring a zero-intensity at its center. The STED beam has the ability to inhibit fluorescence from excited molecules. The higher the STED intensity, the more efficient this inhibitory effect is. As a consequence, fluorescence is confined to a sub-diffraction sized area. The super-resolution image is recorded by scanning this area across the sample.

To assess information beyond the diffraction limit, experiments were performed with a custom-built 3D-STED microscope. The excitation beam of $640 \mathrm{~nm}$ wavelength is emitted from a Picosecond Pulsed Diode Laser Head (LDH-P-C640B, PicoQuant, Berlin, Germany). As STED light source at $775 \mathrm{~nm}$, subnanosecond laser (Katana-08, Onefive GmbH, Zurich, Switzerland) is used. To achieve a super-resolved image in 3D, the Easy3D-STED Module (Abberior Instruments $\mathrm{GmbH}$, Göttingen, Germany) is used: a programmable spatial light modulator (SLM) creates the STED light phase patterns required for 3D STED microscopy. It allowed a lateral (xy) and axial (z) resolution enhancement with only one STED beam instead of two separate STED beams. The relative pulse delay between the excitation laser and the STED laser is set electronically. Laser focusing and fluorescence collection are performed by the same oil immersion objective (UPlanSApo $60 \times 1.35$ Oil, Olympus Corporation, Tokyo, Japan). An appropriate series of dichroic mirrors and optical filters separates the fluorescent signal from both, the excitation and the STED light, and sends it into the detector. Fluorescence photons are detected by a Single Photon Counting module (SPCM-AQRH-13-FC, Excelitas Technologies Corp., Waltham, MA).

Experiments were run with the software ImSpector (MPI für biophysikalische Chemie, Göttingen, Germany); data analysis was performed with ImageJ (U. S. National Institutes of Health, Bethesda, Maryland, USA, http://imagej.nih.gov/ij/).

Atomic Force Microscopy (AFM). For all AFM-experiments, 60,000 cells ( 62 cells per $\mathrm{mm}^{2}$ ) were seeded on an ibidi-treaded $\mu$-dish (81156, Ibidi) for $30 \mathrm{~min}$. Subsequently, the cells were activated with $100 \mathrm{nM}$ PMA. For imaging, an Olympus IX81 microscope was used and steered with an Olympus CellSense Dimension software (v. 3.15). A custom-made heating system integrated into the dish holder enabled the temperature regulation of the sample $\left(T=37 \mathrm{C}^{\circ}\right)$.

For elasticity measurements, a non-conductive silicon nitride tip (MLCT, $f_{0}=$ $10-20 \mathrm{kHz}, k=0.02 \mathrm{~N} \mathrm{~m}^{-1}$, Bruker) was directed by a JPK AFM-head system (JPK Instruments 00996, Nanowizard 3), calibrated according to the manufacturer's instructions (Nanowizard 3 user manual) and kept in the medium to equilibrate the temperature. The tip was slowly approached to the cell via force-feedback recognition and positioned above the middle of the cell. In general, the sample was measured over a $20 \times 20 \mu \mathrm{m}$ area with $8 \times 8$ force curves for each time point and controlled by a JPK SPM Control software (v.5). To prevent plastic deformation a relative set point of $0.5 \mathrm{nN}$ was chosen together with an extension speed of $3 \mu \mathrm{m} \mathrm{s}^{-1}$ and a delay time of $1 \mathrm{~s}$ between each force curve measurement resulting in a total iteration time of around $6 \mathrm{~min}$. All force curves were manually reviewed, baseline corrected and the Young's modulus $E$ was calculated using the Hertz fit for pyramidal tip geometries

$$
F=\frac{E}{1-\nu^{2}} \frac{\tan (\alpha)}{\sqrt{2}} \delta^{2}
$$

with a Poisson's ratio set to $v=0.5$, a face angle of $\alpha=20^{\circ}$, the measured force $F$ and the tip-sample distance $\delta$ of the force curve. The mean Young's modulus was calculated from the force-curve in the middle of the cell and four direct neighbors to avoid edge effects.

Parallel imaging to verify NETosis and healthiness of the cells were performed on the same system. Here, cells were stained before with $1.62 \mu \mathrm{M}$ Hoechst (SigmaAldrich) and observed with an Orca Flash 2.8 camera (C11440, Hamamatsu) at $\times 40$ magnification (LUCPlan FLN, Olympus). For illumination, an integrated lamp system (cellTIRF-4Line System + IUX-C2, Olympus) was used and filtered by a DAPI filter set (\#86-370-OLY, Olympus).

To measure tether tension during NETosis, the baselines of all AFM retraction curves on the cell were analyzed for step like deflection changes that indicate tether formation (sharp increase of cantilever deflection and constant values before and after). All restoring forces were measured with a JPK Data Processing Software (V. spm 5.0.69, JPK Instruments) and stored separately for each frame time $t$. The membrane tension $T(t)$ was then calculated by using the relation ${ }^{66-68}$

$$
T(t)=\frac{F(t)^{2}}{8 B \pi^{2}}
$$

with $B$ representing the bending stiffness of a lipid membrane (set to $3 \times 10^{-12}$ dyne $\mathrm{cm}$ ) and $F(t)$ the respective tether force ${ }^{2}$. For each frame, these values were averaged $(n \approx 3)$ and each data set was normalized to its first value $T\left(t_{0}\right)$ to enable comparisons between varying base values of control cells.
For pressure measurements, a tipless cantilever (MLCT-O10, $f_{0}=10-20 \mathrm{kHz}$ $k=0.03 \mathrm{~N} \mathrm{~m}^{-1}$, Bruker) was chosen in order to enlarge the contact area of the probed cell. Neutrophils were seeded according to the mentioned protocol, activated with PMA and subsequently incubated for $90 \mathrm{~min}$ at $37^{\circ} \mathrm{C}, 5 \% \mathrm{CO}_{2}$ to ensure unmodified cell properties before cell rupture. Afterwards, cells were placed into the aforementioned setup and the cantilever was manually approached to a single cell using $0.2 \mu \mathrm{m}$ driving steps of the $\mathrm{z}$-piezo motor until cell contact (deflection increase of the cantilever) could be observed. Henceforth, the height of the z-piezo was set constant and the pushing forces of the swelling cell were passively quantified using the Live Tracker function of the JPK SPM Contro software (v.5, JPK Instruments). When the pushing forces reached maximal measurable values of the sensor consecutively, the amount of deflection had to be manually reset by readjusting the alignment mirror (reallocation of laser spot on the sensor). This resulted in a vast decline of the deflection data at certain times (dashed lines in Supplementary Fig. 8d), however an effect on the following process could not be observed. Measurements were continued until a rupture of the cellula membrane occurred which was proven afterwards by both a fast breakdown of the deflection data, as well as propidium iodide stainings (Sigma-Aldrich, $c \approx 1 \mu \mathrm{M}$ ). To calculate the interior pressure of the cell, a phase contrast image of the respective cell was taken directly after reaching the contact point. With this, the contact area $A$ of the cell could be extracted by thresholding the visible cell area and the result was combined with the force $F(t)$ of the deflection measurements to generate the pressure value $p(t)=F(t) / A$

Statistics and data analysis. For data analysis Prism 6 for Mac OS X (v. $6.0 \mathrm{~h}$, GraphPad Software, Inc.), origin (OriginPro8, OriginLab Corporation) and MATLAB (v. R2008a/R2014a; The MathWorks, Inc.) were used.

Statistical analysis was performed with Prism 6 for Mac OS X. For all data sets GAUSS distribution was verified with Shapiro-Wilk normality test if applicable and significance proved by $t$-test or one-/two-way-ANOVA/ Bonferroni's multiple comparisons test (mean \pm standard deviation (SD) or standard error of the mean (SEM); $\left.{ }^{*} p<0.05 ;{ }^{* *} p<0.01 ;{ }^{* *} p<0.001 ;{ }^{* * * *} p<0.0001\right)$. For non-Gaussian distributed data sets Mann-Whitney or Kruskal-Wallis/Dunn's multiple comparisons tests $\left({ }^{*} p<0.05 ;{ }^{* *} p<0.01 ;{ }^{* *} p<0.001 ;{ }^{* * * *} p<0.0001\right)$ were used.

Time-lapse analysis. To quantify the change of chromatin area and eccentricity over the time course of NETosis an image segmentation script was developed in Matlab (v. 2014a). Live-cell images generated by wide field fluorescence microscopy were used for the quantification of the chromatin area. This area is a projected chromatin area because it is derived from $2 \mathrm{D}$ images. CLSM derived time traces were analyzed similarly and are shown for example in Fig. $1 \mathrm{~b}$.

For single cells, areas of high contrast (mainly at the edge between stained regions and the background) were carved out, smoothed and filled to generate an outlined image of each object that could be analyzed. Furthermore, total intensity values were normalized to circumvent problems coming from background fluctuations or different staining intensities. From the resulting area-time curves, four characteristic time points were determined manually as shown in Supplementary Fig. $3 \mathrm{a}: t_{1} /$ start of P2: start of first chromatin area increase, $t_{\mathrm{D}}$ : end of uniform/isotropic area increase in P2, $t_{2}$ : maximal area in P2, $t_{3} /$ start of P3: second start of increase in chromatin area. Hence, the duration of P1 (activation (0 $\min )$ to $\left.t_{1}\right)$ and P2 $\left(t_{1}\right.$ to $\left.t_{3}\right)$, as well as the diffusion coefficient of the expanding chromatin in P2 $\left(t_{1}\right.$ to $\left.t_{\mathrm{D}}\right)$ were calculated. For determination of the diffusion coefficient only cells of population $1\left(t_{2} \neq t_{3}\right)$ were used (see Supplementary Fig. 3a) Quantitative membrane and chromatin analysis (Fig. 6a-d) was based on the same segmentation procedure and extended: For chromatin swelling analysis (Fig. 6a), detected chromatin areas of each frame were binarized first and stacked to produce the shown density plots. Moreover, each frame was divided in a grid of $15 \times 15$ pixel sized sub-windows and analyzed by measuring the average additional or lost chromatin area per window resulting in a growth/shrinking vector for each of these segments. The final vector plot results from averaging all frame vectors of a respective segment. For membrane analysis (Fig. $6 \mathrm{~b}-\mathrm{d}$ ), the position of the rupture point was manually determined from the first frame after membrane burst and then compared with the computed major axis of the fitted cell ellipsoid. Only cells with a clear single rupture point were analyzed.

Pressure calculation. Swelling pressures values shown in Fig. $4 \mathrm{~d}$ were calculated by using an approach motivated by Mazumder et al. ${ }^{52}$ Here, Newtonian propertie of the swelling process were assumed and the Stokes-equation was used (effects of inertia were expected to be negligible)

$$
\nabla \mathbf{p}(t)=\eta \Delta v(t)
$$

In this case, $p(t)$ describes the inherent pressure of the fluid/chromatin network $\eta$ is the viscosity of the liquid and $v(t)$ is the velocity field at time point $t$. The physical problem was simplified in two different ways: First, a radial symmetric force field was assumed $\left(\nabla \mathbf{p} \approx \frac{p}{R}\right)$ and secondly the resistance was assumed to be dominated by viscous forces at the scale of interstices (pores) in the network, which ultimately leads to $\eta \Delta v(t) \approx \frac{\eta}{l_{p}^{2}} \mathrm{~d} R$ with $l_{p}$ describing the average mesh size and $R(t)$ 
the radius of the (chromatin) network. Consequently, it follows

$$
p(t) \approx \frac{\eta(R(t))}{l_{p}^{2}(R(t))} \frac{\mathrm{d} R(t)}{\mathrm{d} t} R(t) .
$$

The exact value of $\eta(R(t))$ is unknown. However, in previous studies the viscosity of the nucleus of an eukaryotic cell before expansion was determined to be $\eta_{0}=0.1 \mathrm{~Pa} \mathrm{~s}^{52}$. It is also known that the viscosity of semiflexible polymer network scales $\eta(R) \sim 1 / R^{3}$ with the overall radius $R$ (correlated to the mesh size) ${ }^{69}$. This relation and $\eta_{0}$ were used to approximate $\eta(R(t))$. Furthermore, to approximate the average mesh size $l_{p}(R)$, a single DNA strain $(L \approx 2 \mathrm{~m}, d \approx 2.2 \mathrm{~nm})$ was arranged into a cubic lattice inside a sphere with a radius $R(t)$. $R(t)$ was measured in the time-lapse movies exemplarily shown in Supplementary Movie 2 using $R(t)=\sqrt{A / \pi})$ and $\frac{\mathrm{d} R(t)}{\mathrm{d} t}$ was calculated by fitting the two dimensional diffusion equation $\frac{\mathrm{d} R(t)}{\mathrm{d} t}=\frac{\mathrm{d}(\sqrt{4 D t})}{\mathrm{d} t}=\frac{4 D}{2 \sqrt{4 D t}}=\sqrt{\frac{D}{t}}=\sqrt{\frac{R^{2}}{4 t^{2}}}=\frac{R}{2 t}$ to the chromatin data between $R\left(t_{1}\right)$ to $R\left(t_{\mathrm{D}}\right)$ and $R\left(t_{\mathrm{D}}\right)$ to $R\left(t_{2}\right)$ and extracting the resulting slope.

Code availability. All Matlab codes used to analyze data are available from the corresponding authors upon request.

\section{Data availability}

Data supporting the findings of this manuscript are available from the corresponding authors upon request.

Received: 2 January 2018 Accepted: 11 August 2018

Published online: 14 September 2018

\section{References}

1. Mantovani, A., Cassatella, M. A., Costantini, C. \& Jaillon, S. Neutrophils in the activation and regulation of innate and adaptive immunity. Nat. Rev. Immunol. 11, 519-531 (2011).

2. Brinkmann, V. et al. Neutrophil extracellular traps kill bacteria. Science $\mathbf{3 0 3}$ 1532-1535 (2004).

3. Brinkmann, V. \& Zychlinsky, A. Neutrophil extracellular traps: is immunity the second function of chromatin? J. Cell. Biol. 198, 773-783 (2012).

4. Fuchs, T. A. et al. Novel cell death program leads to neutrophil extracellular traps. J. Cell. Biol. 176, 231-241 (2007).

5. Metzler, K. D. et al. Myeloperoxidase is required for neutrophil extracellular trap formation: implications for innate immunity. Blood 117, 953-959 (2011).

6. von Kockritz-Blickwede, M. et al. Phagocytosis-independent antimicrobial activity of mast cells by means of extracellular trap formation. Blood 111, 3070-3080 (2008)

7. Ueki, S. et al. Eosinophil extracellular DNA trap cell death mediates lytic release of free secretion-competent eosinophil granules in humans. Blood 121, 2074-2083 (2013).

8. Zhang, X., Zhuchenko, O., Kuspa, A. \& Soldati, T. Social amoebae trap and kill bacteria by casting DNA nets. Nat. Commun. 7, 10938 (2016).

9. Hawes, M. C. et al. Extracellular DNA: the tip of root defenses? Plant Sci. 180 741-745 (2011).

10. Papayannopoulos, V., Metzler, K. D., Hakkim, A. \& Zychlinsky, A. Neutrophil elastase and myeloperoxidase regulate the formation of neutrophil extracellular traps. J. Cell. Biol. 191, 677-691 (2010).

11. Garcia-Romo, G. S. et al. Netting neutrophils are major inducers of type I IFN production in pediatric systemic lupus erythematosus. Sci. Transl. Med. 3, 73ra20 (2011).

12. Sorensen, O. E. et al. Papillon-Lefevre syndrome patient reveals speciesdependent requirements for neutrophil defenses. J. Clin. Invest. 124, 4539-4548 (2014).

13. Martinod, K. et al. PAD4-deficiency does not affect bacteremia in polymicrobial sepsis and ameliorates endotoxemic shock. Blood $\mathbf{1 2 5}$, 1948-1956 (2015).

14. Erpenbeck, L., Schon, M. P. Neutrophil extracellular traps: protagonists of cancer progression? Oncogene 36, 2483 (2016)

15. Brill, A. et al. Neutrophil extracellular traps promote deep vein thrombosis in mice. J. Thromb. Haemost. 10, 136-144 (2012).

16. Savchenko, A. S. et al. VWF-mediated leukocyte recruitment with chromatin decondensation by PAD4 increases myocardial ischemia/reperfusion injury in mice. Blood 123, 141-148 (2014).

17. Kolaczkowska, E. et al. Molecular mechanisms of NET formation and degradation revealed by intravital imaging in the liver vasculature. Nat. Commun. 6, 6673 (2015).
18. Gupta, A. K., Hasler, P., Holzgreve, W., Gebhardt, S. \& Hahn, S. Induction of neutrophil extracellular DNA lattices by placental microparticles and IL- 8 and their presence in preeclampsia. Hum. Immunol. 66, 1146-1154 (2005).

19. Sur Chowdhury, C. et al. Enhanced neutrophil extracellular trap generation in rheumatoid arthritis: analysis of underlying signal transduction pathways and potential diagnostic utility. Arthritis Res. Ther. 16, R122 (2014).

20. Hoppenbrouwers, T. et al. In vitro induction of NETosis: comprehensive live imaging comparison and systematic review. PLOS ONE 12, e0176472 (2017).

21. Kamoshida, G. et al. Pathogenic Bacterium Acinetobacter baumannii Inhibits the Formation of Neutrophil Extracellular Traps by Suppressing Neutrophil Adhesion. Front. Immunol. 9, 178 (2018).

22. Stavrou, E. X. et al. Factor XII and uPAR upregulate neutrophil functions to influence wound healing. J. Clin. Invest. 128, 944-959 (2018).

23. O'Brien, X. M. \& Reichner, J. S. Neutrophil integrins and matrix ligands and NET release. Front. Immunol. 7, 363 (2016)

24. $\mathrm{Xu}, \mathrm{Z}$. et al. Interaction of kindlin-3 and beta2-integrins differentially regulates neutrophil recruitment and NET release in mice. Blood 126, 373-377 (2015).

25. Clark, S. R. et al. Platelet TLR4 activates neutrophil extracellular traps to ensnare bacteria in septic blood. Nat. Med. 13, 463-469 (2007).

26. Maugeri, N. et al. Activated platelets present high mobility group box 1 to neutrophils, inducing autophagy and promoting the extrusion of neutrophil extracellular traps. J. Thromb. Haemost. 12, 2074-2088 (2014).

27. Yu, X., Tan, J. \& Diamond, S. L. Hemodynamic force triggers rapid NETosis within sterile thrombotic occlusions. J. Thromb. Haemost. 16, 316-329 (2018).

28. Kenny, E. F. et al. Diverse stimuli engage different neutrophil extracellular trap pathways. eLife 6, e24437 (2017)

29. Brinkmann, V., Goosmann, C., Kuhn, L. I. \& Zychlinsky, A. Automatic quantification of in vitro NET formation. Front. Immunol. 3, 413 (2012).

30. van der Linden, M., Westerlaken, G. H. A., van der Vlist, M., van Montfrans, J \& Meyaard, L. Differential signalling and kinetics of neutrophil extracellular trap release revealed by quantitative live imaging. Sci. Rep. 7, 6529 (2017).

31. Gupta, S., Chan, D. W., Zaal, K. J. \& Kaplan, M. J. A high-throughput realtime imaging technique to quantify NETosis and distinguish mechanisms of cell death in human neutrophils. J. Immunol. 200, 869-879 (2018).

32. Elacqua, J. J., McGregor, A. L. \& Lammerding, J. Automated analysis of cell migration and nuclear envelope rupture in confined environments. PLOS ONE 13, e0195664 (2018)

33. Amulic, B. et al. Cell-cycle proteins control production of neutrophil extracellular traps. Develop. Cell 43, 449-462 (2017).

34. Metzler, K. D., Goosmann, C., Lubojemska, A., Zychlinsky, A. \& Papayannopoulos, V. A myeloperoxidase-containing complex regulates neutrophil elastase release and actin dynamics during NETosis. Cell Rep. 8, 883-896 (2014)

35. Borregaard, N. \& Herlin, T. Energy metabolism of human neutrophils during phagocytosis. J. Clin. Invest. 70, 550-557 (1982)

36. Chacko, B. K. et al. Methods for defining distinct bioenergetic profiles in platelets, lymphocytes, monocytes, and neutrophils, and the oxidative burst from human blood. Lab. Investig. 93, 690-700 (2013).

37. Wick, A. N., Drury, D. R., Nakada, H. I. \& Wolfe, J. B. Localization of the primary metabolic block produced by 2-deoxyglucose. J. Biol. Chem. 224, 963-969 (1957)

38. Chen, W. \& Gueron, M. The inhibition of bovine heart hexokinase by 2deoxy-D-glucose-6-phosphate: characterization by 31P NMR and metabolic implications. Biochimie 74, 867-873 (1992).

39. Lane, T. A. \& Lamkin, G. E. A reassessment of the energy requirements for neutrophil migration: adenosine triphosphate depletion enhances chemotaxis. Blood 64, 986-993 (1984).

40. Azevedo, E. P. et al. A metabolic shift toward pentose phosphate pathway is necessary for amyloid fibril- and phorbol 12-Myristate 13-Acetate-induced Neutrophil Extracellular Trap (NET) formation. J. Biol. Chem. 290, 22174-22183 (2015)

41. Pitanga, T. N. et al. Neutrophil-derived microparticles induce myeloperoxidase-mediated damage of vascular endothelial cells. BMC Cell. Biol. 15, 21 (2014)

42. Bowler, M. W., Montgomery, M. G., Leslie, A. G., Walker, J. E. How azide inhibits ATP hydrolysis by the F-ATPases. Proc. Natl Acad. Sci. USA 103(23), 8646-8649 (2006)

43. Merrill, G. A., Bretthauer, R., Wright-Hicks, J. \& Allen, R. C. Effects of inhibitors on chicken polymorphonuclear leukocyte oxygenation activity measured by use of selective chemiluminigenic substrates. Comp. Med. 51, 16-21 (2001).

44. Kettle, A. J., Gedye, C. A. \& Winterbourn, C. C. Mechanism of inactivation of myeloperoxidase by 4 -aminobenzoic acid hydrazide. Biochem. J. 321(Pt 2), 503-508 (1997).

45. Kettle, A. J., Gedye, C. A., Hampton, M. B. \& Winterbourn, C. C. Inhibition of myeloperoxidase by benzoic acid hydrazides. Biochem. J. 308(Pt 2), 559-563 (1995).

46. Milo, R., Phillips, R. Cell Biology by the Numbers (Garland Science, 2016) 
47. Prazeres, D. M. F. Prediction of diffusion coefficients of plasmids. Biotechnol. Bioeng. 99, 1040-1044 (2008)

48. Luby-Phelps, K. et al. A novel fluorescence ratiometric method confirms the low solvent viscosity of the cytoplasm. Biophys. J. 65, 236-242 (1993).

49. Wang, Y. et al. Histone hypercitrullination mediates chromatin decondensation and neutrophil extracellular trap formation. J. Cell. Biol. 184, 205-213 (2009).

50. Latulippe, D. R. \& Zydney, A. L. Radius of gyration of plasmid DNA isoforms from static light scattering. Biotechnol. Bioeng. 107, 134-142 (2010).

51. Tree, D. R., Muralidhar, A., Doyle, P. S. \& Dorfman, K. D. Is DNA a good model polymer? Macromolecules 46, 8369-8382 (2013).

52. Mazumder, A., Roopa, T., Basu, A., Mahadevan, L. \& Shivashankar, G. V. Dynamics of chromatin decondensation reveals the structural integrity of a mechanically prestressed nucleus. Biophys. J. 95, 3028-3035 (2008).

53. Koslov, M. M. \& Markin, V. S. A theory of osmotic lysis of lipid vesicles. J Theor. Biol. 109, 17-39 (1984).

54. Stewart, M. P. et al. Hydrostatic pressure and the actomyosin cortex drive mitotic cell rounding. Nature 469, 226-230 (2011).

55. Gabler, W. L., Bullock, W. W. \& Creamer, H. R. Phorbol myristate acetate induction of chemotactic migration of human polymorphonuclear neutrophils. Inflammation 17, 521-530 (1993).

56. Bengtsson, T., Orselius, K. \& Wettero, J. Role of the actin cytoskeleton during respiratory burst in chemoattractant-stimulated neutrophils. Cell. Biol. Int. 30, 154-163 (2006)

57. Neeli, I., Dwivedi, N., Khan, S. \& Radic, M. Regulation of extracellular chromatin release from neutrophils. J. Innate Immun. 1, 194-201 (2009).

58. Chen, K. W. et al. The neutrophil NLRC4 inflammasome selectively promotes IL-1beta maturation without pyroptosis during acute Salmonella challenge. Cell Rep. 8, 570-582 (2014).

59. Pierre, S. \& Julie, P. Membrane tension and cytoskeleton organization in cell motility. J. Phys. 27, 273103 (2015).

60. Diz-Muñoz, A., Fletcher, D. A. \& Weiner, O. D. Use the force: membrane tension as an organizer of cell shape and motility. Trends Cell Biol. 23, 47-53 (2013)

61. Kruss, S. et al. Adhesion maturation of neutrophils on nanoscopically presented platelet glycoprotein Ibalpha. ACS Nano 7, 9984-9996 (2013).

62. Kruss, S., Erpenbeck, L., Schon, M. P. \& Spatz, J. P. Circular, nanostructured and biofunctionalized hydrogel microchannels for dynamic cell adhesion studies. Lab. Chip. 12, 3285-3289 (2012).

63. Fischer-Friedrich, E., Hyman, A. A., Julicher, F., Muller, D. J. \& Helenius, J. Quantification of surface tension and internal pressure generated by single mitotic cells. Sci. Rep. 4, 6213 (2014).

64. Brinkmann, V., Laube, B., Abu Abed, U., Goosmann, C., Zychlinsky, A. Neutrophil extracellular traps: how to generate and visualize them. J. Vis. Exp. 36, e1724 (2010).

65. Hell, S. W. \& Wichmann, J. Breaking the diffraction resolution limit by stimulated emission: stimulated-emission-depletion fluorescence microscopy. Opt. Lett. 19, 780-782 (1994)

66. Sheetz, M. P. Cell control by membrane-cytoskeleton adhesion. Nat. Rev. Mol. Cell Biol. 2, 392-396 (2001).

67. Waugh, R. E., Song, J., Svetina, S. \& Zeks, B. Local and nonlocal curvature elasticity in bilayer membranes by tether formation from lecithin vesicles. Biophys. J. 61, 974-982 (1992).
68. Hochmuth, R. M., Mohandas, N. \& Blackshear, P. L. Jr Measurement of the elastic modulus for red cell membrane using a fluid mechanical technique. Biophys. J. 13, 747-762 (1973)

69. Broedersz, C. P. \& MacKintosh, F. C. Modeling semiflexible polymer networks. Rev. Mod. Phys. 86, 995-1036 (2014).

\section{Acknowledgements}

This project was supported by the state of Lower Saxony (life@nano) and the German Research Foundation (DFG grant KR 4242/4-1 and ER 723/2-1). We acknowledge financial support by the open-access funding program of the German Research Foundation and the publication funds of the University Medical Center (UMG). Part of this work was supported by the Cluster of Excellence and DFG Research Center Nanoscale Microscopy and Molecular Physiology of the Brain (CNMPB). We thank Elisa D'Este and Grazvydas Lukinavicius for input on SiR-Hoechst stainings. We thank Andreas Janshoff and Claudia Steinem for fruitful discussions and support. We are grateful for fruitful discussions about active matter with members of the collaborative research center SFB 937 funded by the DFG.

\section{Author contributions}

S.K. and L.E. conceived the study with inputs from M.P.S. E.N. and D.M. performed experiments. E.N., F.R., C.G. and A.E. performed STED microscopy. G.G., A.K.T., J.G. and S.S.S. performed additional staining and inhibitor experiments. E.N., D.M., L.E. and S.K. analyzed data/images. E.N., D.M., L.E., and S.K. wrote the manuscript with inputs from all authors.

\section{Additional information}

Supplementary Information accompanies this paper at https://doi.org/10.1038/s41467018-06263-5.

Competing interests: The authors declare no competing interests.

Reprints and permission information is available online at http://npg.nature.com/ reprintsandpermissions/

Publisher's note: Springer Nature remains neutral with regard to jurisdictional claims in published maps and institutional affiliations.

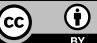

Open Access This article is licensed under a Creative Commons Attribution 4.0 International License, which permits use, sharing, adaptation, distribution and reproduction in any medium or format, as long as you give appropriate credit to the original author(s) and the source, provide a link to the Creative Commons license, and indicate if changes were made. The images or other third party material in this article are included in the article's Creative Commons license, unless indicated otherwise in a credit line to the material. If material is not included in the article's Creative Commons license and your intended use is not permitted by statutory regulation or exceeds the permitted use, you will need to obtain permission directly from the copyright holder. To view a copy of this license, visit http://creativecommons.org/ licenses/by/4.0/.

() The Author(s) 2018 


\section{Supplementary information}

?

\section{Chromatin swelling drives neutrophil extracellular trap release}

Elsa Neubert ${ }^{1,2^{*}}$, Daniel Meyer ${ }^{2,3^{*}}$, Francesco Rocca ${ }^{4}$, Gökhan Günay ${ }^{1,2}$, Anja Kwaczala-Tessmann ${ }^{1}$, Julia

Grandke $^{1}$, Susanne Senger Sander ${ }^{1}$, Claudia Geisler ${ }^{3,4}$, Alexander Egner ${ }^{3,4}$, Michael P. Schön ${ }^{1,5}$, Luise

Erpenbeck $^{1 *+}$, Sebastian Kruss ${ }^{2,3 *+}$

${ }^{1}$ Department of Dermatology, Venereology and Allergology, University Medical Center, Goettingen University, Germany

${ }^{2}$ Institute of Physical Chemistry, Goettingen University, Germany

${ }^{3}$ Center for Nanoscale Microscopy and Molecular Physiology of the Brain (CNMPB), Goettingen, Germany

${ }^{4}$ Optical Nanoscopy, Laser-LaboratoriumGoettingene.V., Germany

${ }^{5}$ Lower Saxony Institute of Occupational Dermatology, University Medical Center Goettingen and University of Osnabrück, Germany

*These authors contributed equally

${ }^{+}$Correspondence should be addressed to L.E. or S.K. (email: luise.erpenbeck@med.uni-goettingen or skruss@uni-goettingen.de) 
a

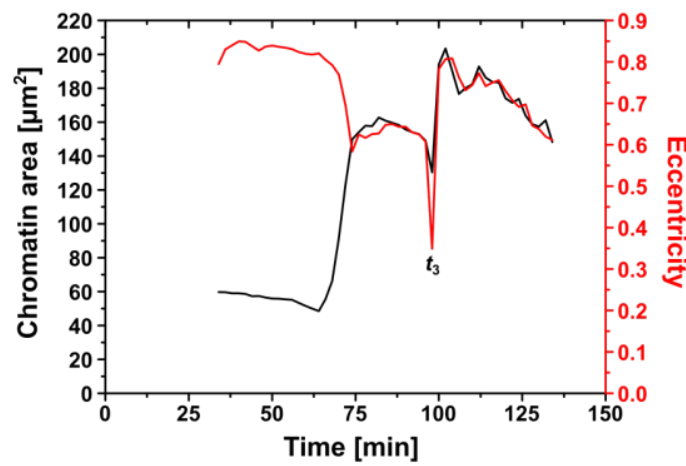

b

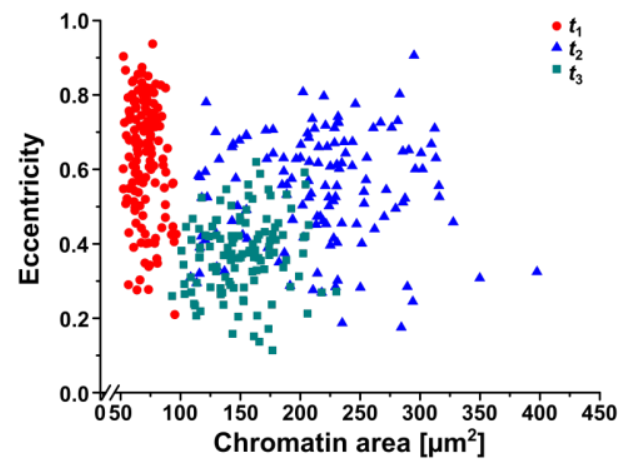

Supplementary Fig. 1: Eccentricity of chromatin during NETosis. Chromatin rounds up during Phase 1 and 2 and reaches maximal circularity (minimal eccentricity) exactly before NET release $\left(t_{3}\right)$. $\boldsymbol{a}$, Eccentricity over time displayed for the example cell of Fig. 1 b (Supplementary Movie 2). b, Comparison of the eccentricity at different time points $\left(t_{1}, t_{2}, t_{3}\right)$ for in total 139 cells of five donors. 

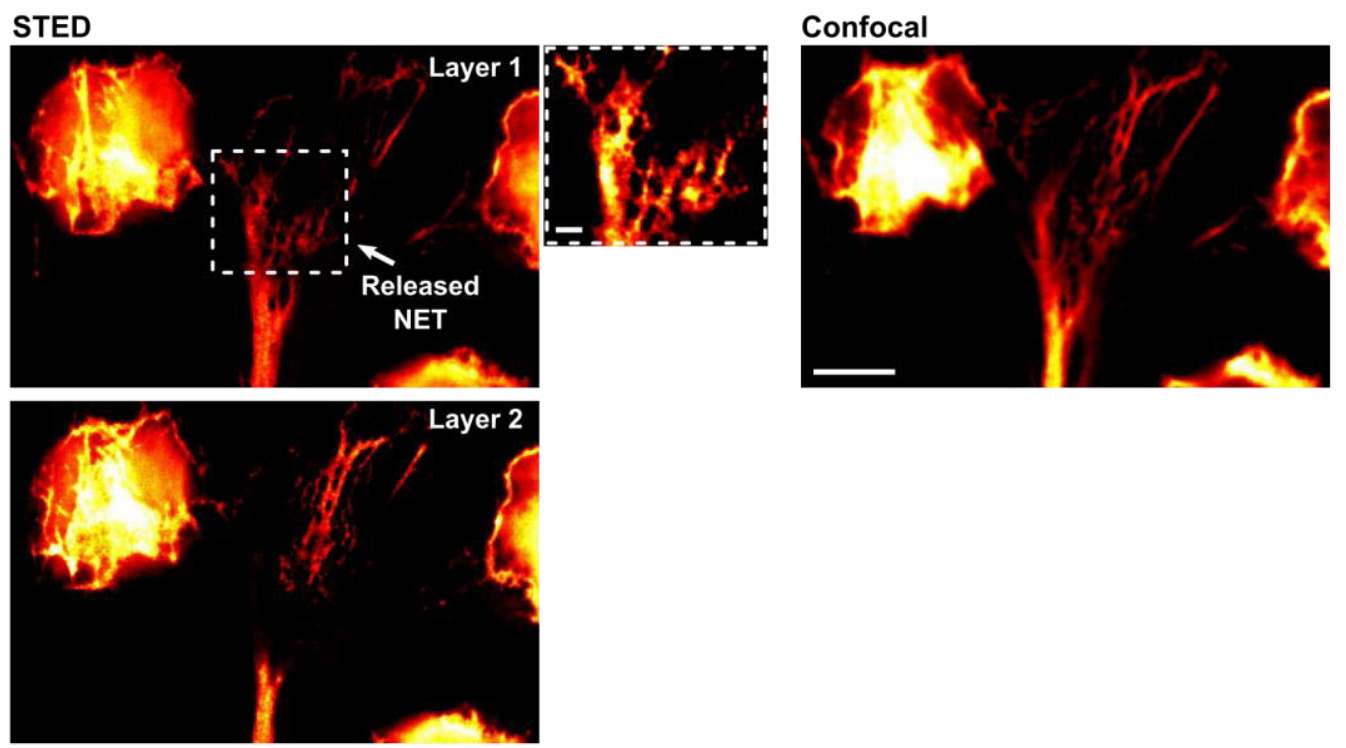

Supplementary Fig. 2: STED nanoscopy of NET fine structure. Images of PMA-activated neutrophils (arrow $=$ released NET) in comparison to confocal microscopy. Staining: SiR-DNA on fixed samples. Scale $=5 \mu \mathrm{m}$ and $1 \mu \mathrm{m}$ (insert). 
a
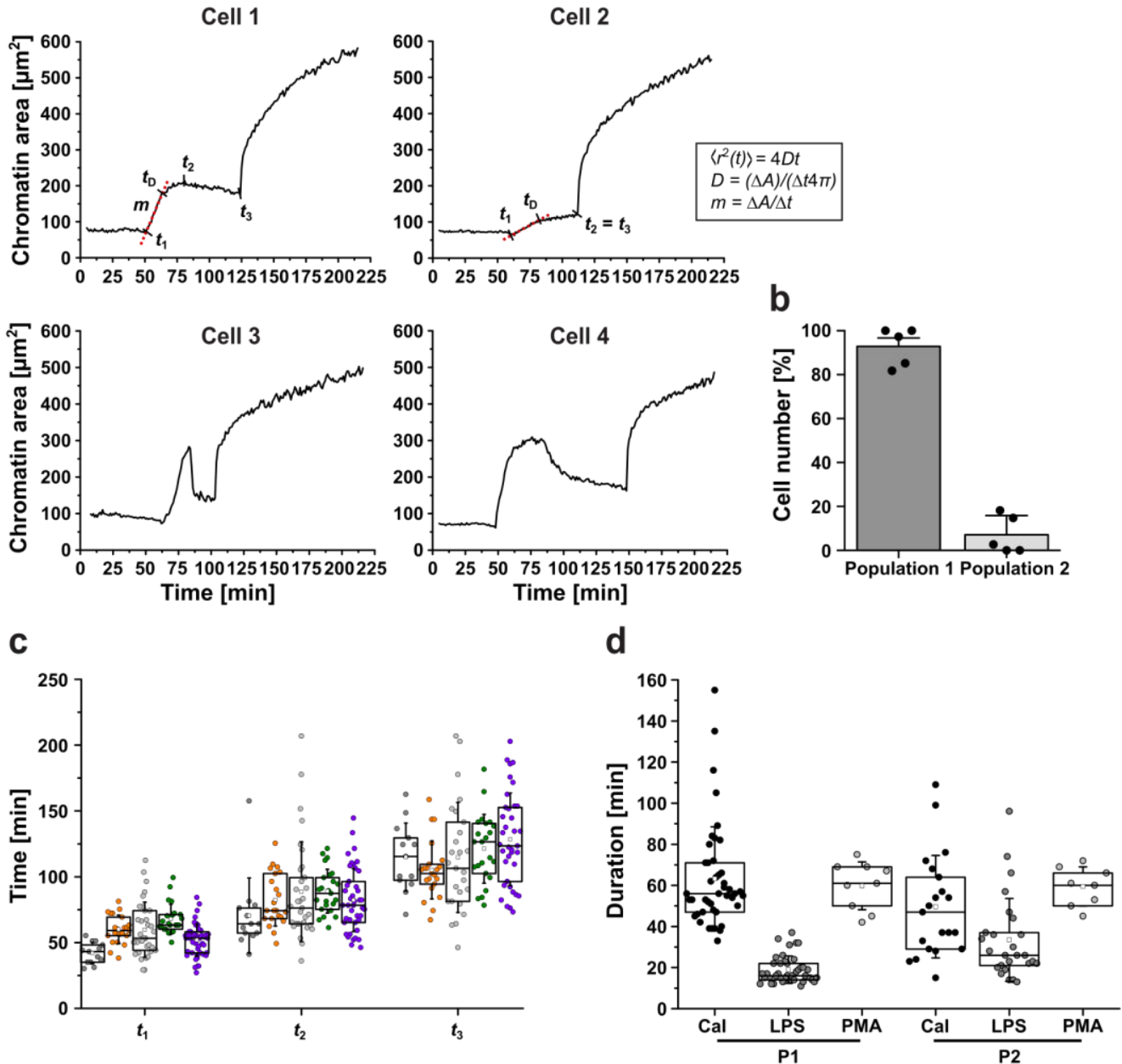

Supplementary Fig. 3: Characterization of phases of NETosis. a, b Time course of changes in chromatin area during PMA-induced NETosis displayed for different cells imaged with wide field fluorescence microscopy. Cells can be divided in two different populations based on their chromatin time course. Cells of population 1 show a maximum of chromatin expansion $\left(t_{2}\right)$ in P2. Chromatin of cells of population 2 expands until cell rupture at $t_{3} . t_{2}$ cannot be defined for this population (cell 2). Cell 1, 3 and 4 represent cells of population 1 and cell 2 of population 2. Therefore, only population 1 (>90\% of cells) has been used to analyze the correlation between cell area at rupture/pressure $P$ at $t_{2}$ and the time between $t_{2}$ and $t_{3}$ (rupture delay time) (Fig. 4a, d). The diffusion coefficient shown in Supplementary Fig. S8a is calculated based on the slope of the linear fit from $t_{1}$ to $t_{\mathrm{D}}$. Mean $\pm S E M$. c, Distribution of the time points $t_{1}, t_{2}$ and $t_{3}$ of all cells of five individual experiments (summary shown in Fig. 1c). Time points were calculated based on time-lapse movies recorded with conventional fluorescence microscopy. $N=5$. d, Kinetics of NET formation after stimulation with three different stimuli $\left(P M A=100 \mathrm{nM}, L P S=25 \mu \mathrm{g} \mathrm{ml} \mathrm{l}^{-1}, \mathrm{CaI}=4 \mu \mathrm{M}\right)$. Duration of $P 1$ is significantly shortened after stimulation with LPS compared to PMA and CaI, whereas P2 displays only small stimulus dependence. $N=1$. Boxplots $(\boldsymbol{c}, \boldsymbol{d})$ display the 25 th and 75 th percentile with the horizontal line at the median, squares represent the mean and whiskers the $S D$. 
a
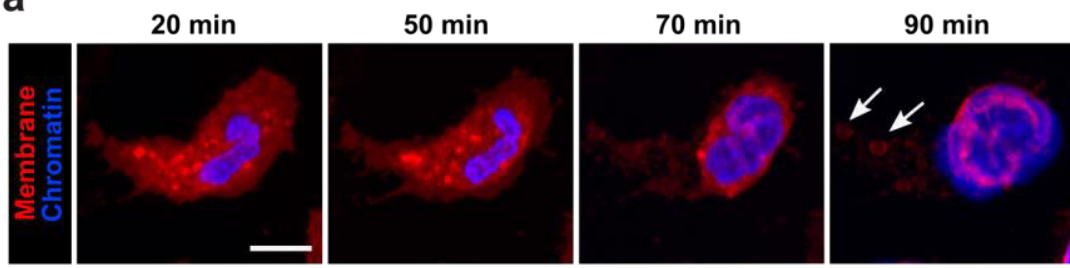

$120 \mathrm{~min}$

b
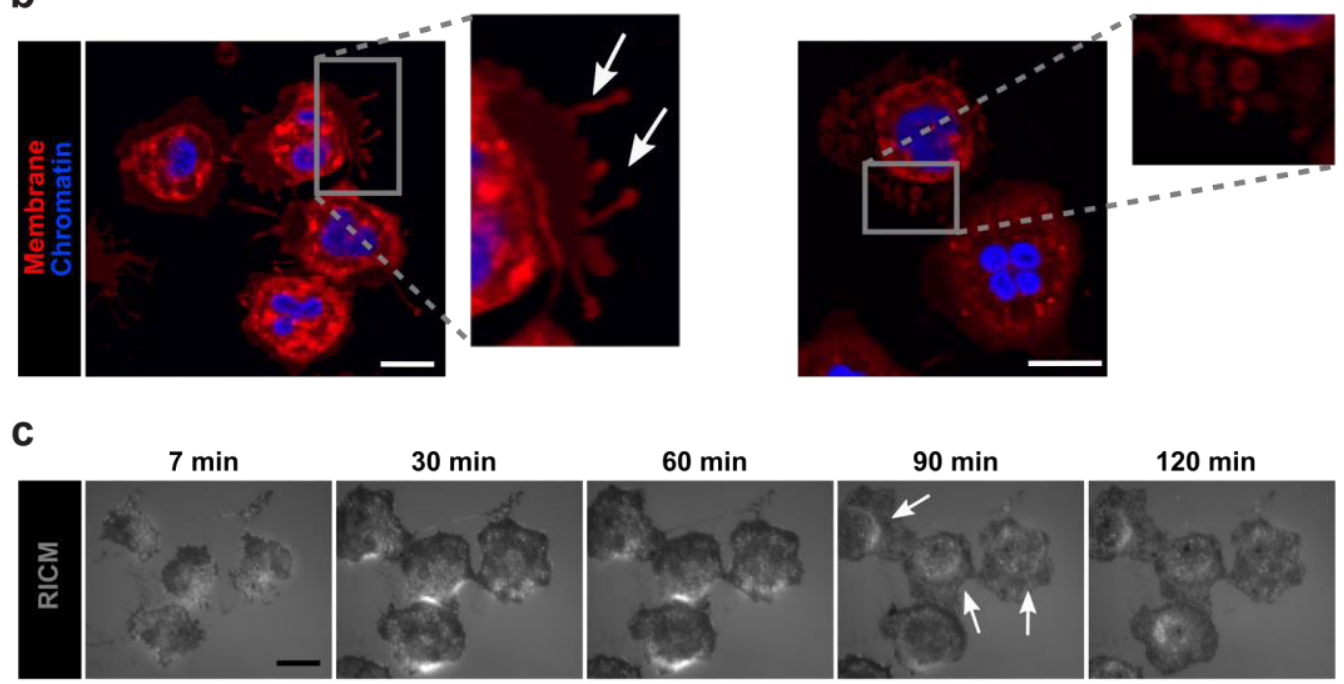

$120 \mathrm{~min}$
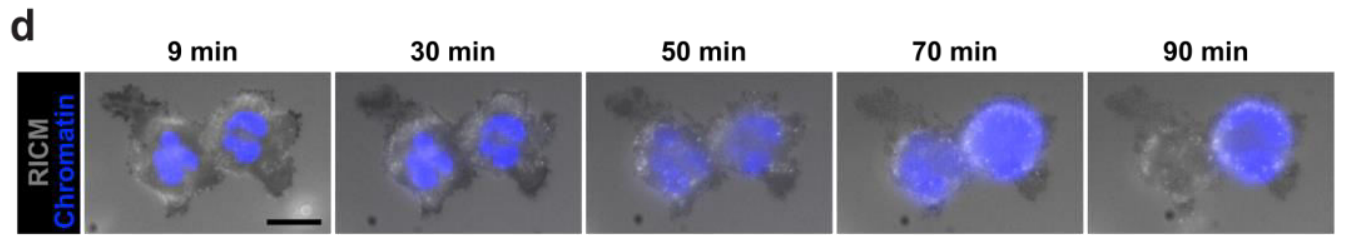

Supplementary Fig. 4: Membrane rearrangement during NETosis. a, CLSM images of a human neutrophil undergoing NETosis (blue $=$ Hoechst 33342/DNA, red $=$ PKH26/membrane) observed by $3 D-C L S M$ (see also Supplementary Movie 8). The cell spreads initially but retracts its cell body leaving membrane behind. Vesicles are formed closely bound to the substrate, probably originating from excess membrane (arrows, $t=90 \mathrm{~min}$ ). Scale $=10 \mu \mathrm{m} . \boldsymbol{b}$, Characteristic behavior of the cell membrane during NET formation observed by time-lapse CLSM (blue $=D N A$, red $=$ membrane). Cells form membrane extensions (arrows) in late P1 (left) and membrane vesicles at the surface during the cell rounding process in P2 (right). Scale $=10 \mu \mathrm{m} . c$, Representative images of human neutrophils undergoing PMA-induced NET formation recorded with real-time reflection interference contrast microscopy (RICM) on glass. Images allow the label-free analysis of the cell/surface contact area (black $=$ cell closer to the surface, white $=$ further away from the surface). During NETosis, the cells round up, leave membrane closely bound to the substrate behind (arrows, $t=90 \mathrm{~min}$ ) and expel the NET (see also Supplementary Movie 9). Scale $=10 \mu \mathrm{m}$. d, Overlay of real-time RICM with immunofluorescence (blue $=$ chromatin) to verify $N E T$ release $(t=70,90 \mathrm{~min})$ during time-lapse RICM imaging. Scale $=10 \mu \mathrm{m}$. 
?
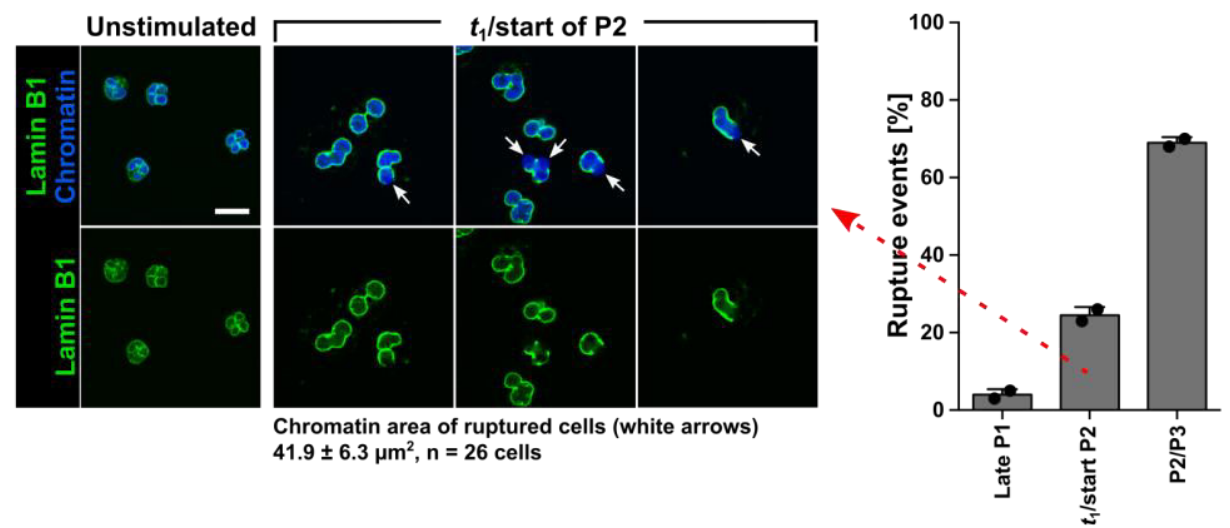

Supplementary Fig. 5: Rupture of the nuclear envelope at beginning of P2. Overlay of chromatin (blue) and lamin B1 (green) of unstimulated and NETotic human neutrophil (CLSM, fixed samples). Average chromatin area at rupture point of the surrounding lamin $B 1\left(t_{1}\right)$ is $41.9 \pm 6.3 \mu m^{2}(n=26$ cells from two donors) and in good agreement with the chromatin area at $t_{1}$ determined during live cell imaging (Fig. 1a, b). At this time point, the nuclear envelope rupture events increase $\left(t_{1} /\right.$ start P2) until a high rate of rupture events (small whole up to full loss of the Lamin B1 surrounding in P2 and P3). Calculation based on CLSM images of fixed samples. $N=2$ donors (100 cells per condition and N). Mean $\pm S D$. Scale $=10 \mu \mathrm{m}$ 
a

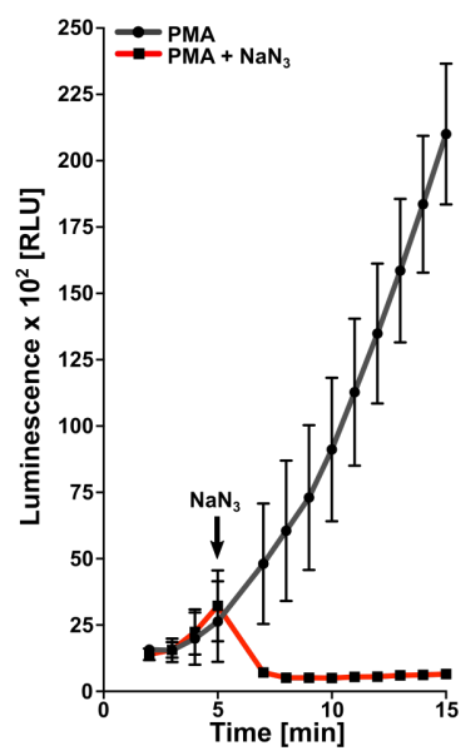

b

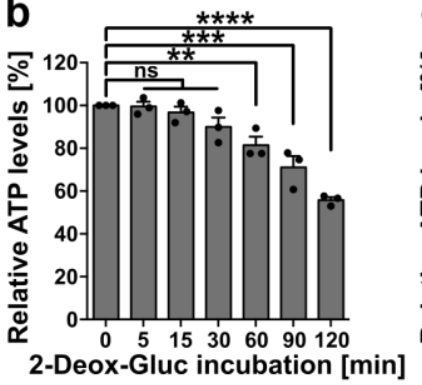

d

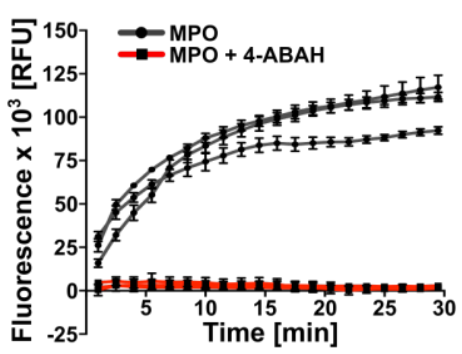

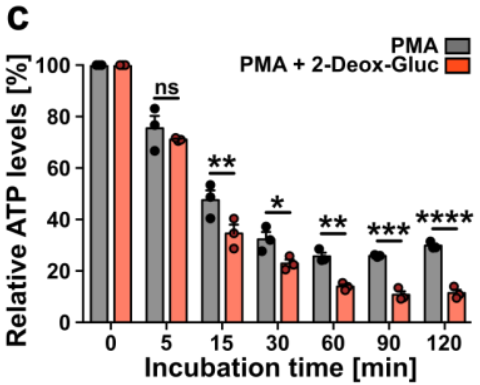

Seeded cells

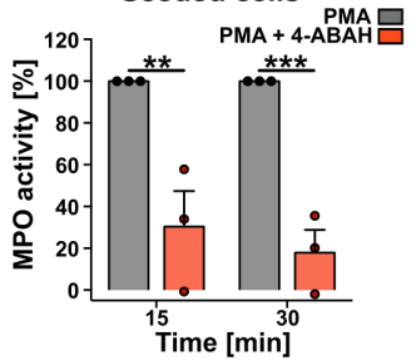

Supplementary Fig. 6: Inhibitory effect of $\mathrm{NaN}_{3}$. 2-Deox-Gluc and 4-ABAH on NETotic cells. a, Effect of $3 \mathrm{mM}$ sodium azide $\left(\mathrm{NaN}_{3}\right)$ on PMA (100 $\mathrm{nM}$ )-induced ROS production of human neutrophils determined by chemiluminescence of luminol. NaN $\mathrm{N}_{3}$ inhibits ROS directly after addition and enables a stable inhibition for at least $30 \mathrm{~min}$, as shown in Supplementary Fig. 9c. Experimental setup comparable with the setup used for the experiments shown in Fig. $3 \boldsymbol{b} . N=1$ (triplicates). Mean $\pm S D$. b, ATP levels of unstimulated neutrophils after incubation with $5 \mathrm{mM}$ 2-Deoxy-glucose (2-Deox-Gluc) for different time intervals. 2-Deox-Gluc reduces ATP levels already after short incubation and significantly after more than 60 min compared to untreated cells $(t=0 \mathrm{~min})$. Statistics: One-way ANOVA (Bonferroni's multiple comparison test, ${ }^{* *} p<0.01,{ }^{* * *} p<0.001,{ }^{* * * *} p<0.0001$ ). $N=3$. Mean \pm SEM. c, ATP levels of PMA (100 nM)-stimulated neutrophils with and without incubation with $5 \mathrm{mM}$ 2-Deox-Gluc for different time periods. 2-Doxy-Gluc significantly decreases the ATP levels after more than 15 min PMA stimulation compared to exclusive PMA treatment $(t=0 \mathrm{~min})$. PMA stimulation alone decreases ATP levels by more than $70 \%$. Experimental setup comparable with the setup used in Fig. 3b. Statistics: Two-way ANOVA (Bonferroni's multiple comparisons test, *p $<0.05$, $\left.{ }^{* *} p<0.01,{ }^{* * *} p<0.001,{ }^{* * * * p}<0.0001\right) . N=3$. Mean \pm SEM. d, inhibitory effect of $100 \mu M 4-$ aminobenzoic acid hydrazide (4-ABAH) on MPO activity. left, 4-ABAH inhibits purified MPO significantly and stable after $1 \mathrm{~min}$ for at least $30 \mathrm{~min} . N=3$. Mean $\pm S D$. right, 4-ABAH inhibits PMA (100 nM)-induced MPO activity significantly and stable after 15 min and 30 min cell incubation followed by complete cell lysis for MPO activity measurements. Experimental setup comparable with the setup in Fig. 3b. Statistics: Two-way ANOVA (Bonferroni's multiple comparisons test, **p<0.01, $* * * p<0.001) . N=3$. Mean \pm SEM. 
a

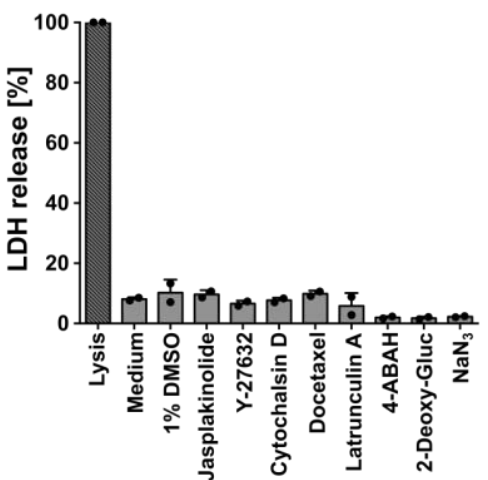

b

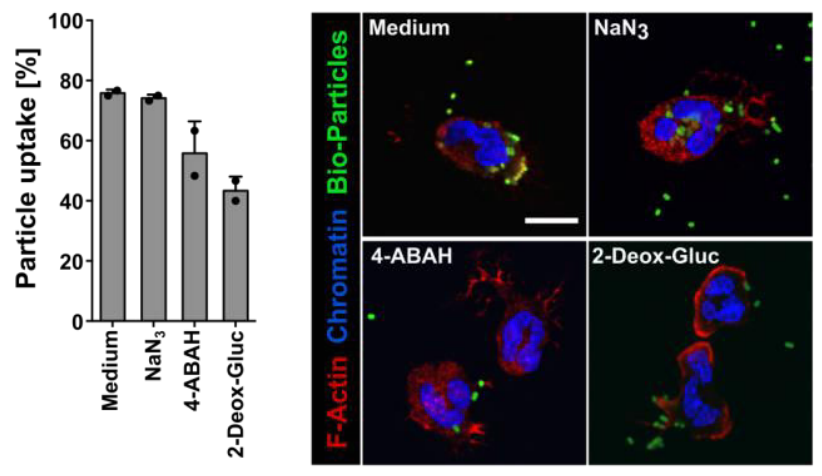

Supplementary Fig. 7: Influence of metabolic and cytoskeletal inhibitors on neutrophil function. a, Toxicity of used inhibitors on human neutrophils detected by release of lactatdehydrogenase (LDH) relative to complete cell lysis. All inhibitors were tested for the concentrations used in this study and the maximal incubation time of $3 \mathrm{~h}$. All inhibitors show less than $10 \%$ cell toxicity, which is below the toxicity of $1 \%$ DMSO with $10.2 \%$ (maximal solvent concentration in inhibitor studies). $N=2$. Mean \pm $\mathrm{SD}$. b, Effect of $\mathrm{NaN}_{3}(3 \mathrm{mM}), 4-\mathrm{ABAH}(100 \mu \mathrm{M})$ and 2-Deox-Gluc (5 mM) on uptake of FITC-labeled E. coli BioParticles. The particle uptake is not affected by $\mathrm{NaN}_{3}$, but clearly decreased in presence of 4-ABAH and 2-Deox-Gluc. Calculation based on confocal imaging of fixed samples after incubation with BioParticles for 30 min. Staining: red $=$ F-actin/Phalloidin555, blue = chromatin/Hoechst, green $=$ FITC-labeled E. coli BioParticles. Scale $=10 \mu \mathrm{m} . N=2(n=60$ cells per condition and $N)$. Mean $\pm S D$.

?

?

?

? 
a

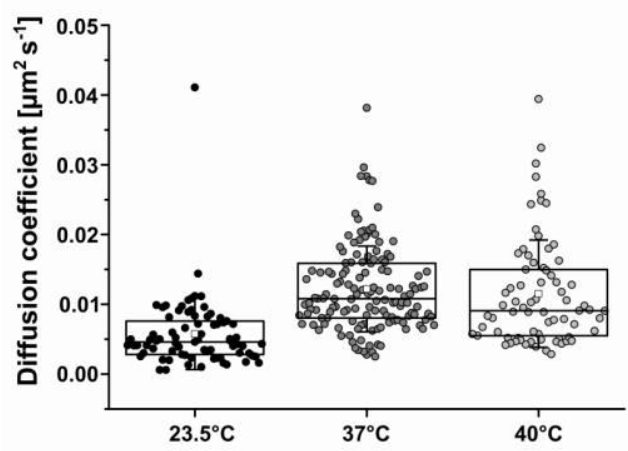

C

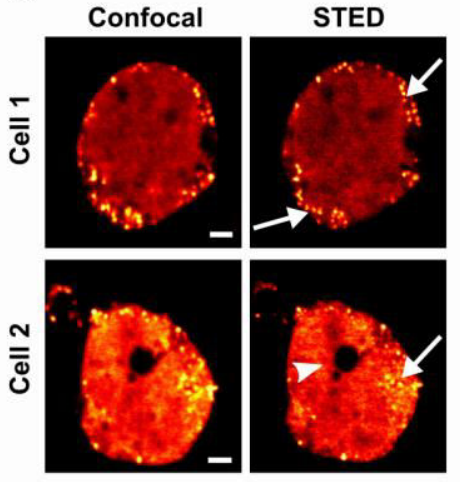

b

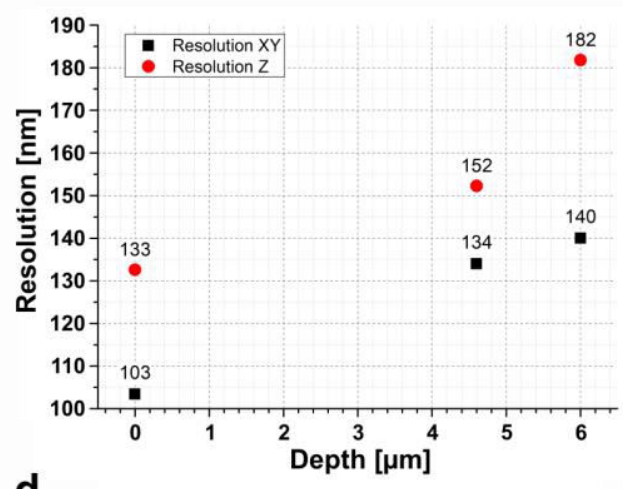

d

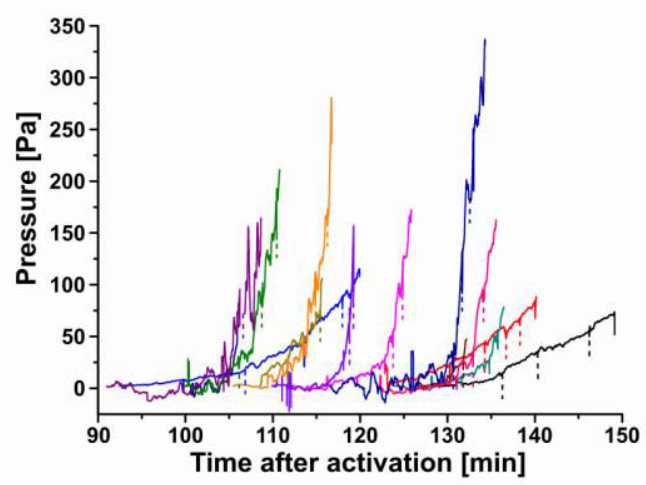

Supplementary Fig. 8: Properties and consequences of chromatin swelling: a, Effective $2 D$ diffusion coefficient of chromatin expansion in $P 2$ ( $t_{1}$ to $\left.t_{\mathrm{D}}\right)$ at different temperatures (Median: $23.5^{\circ} \mathrm{C}$

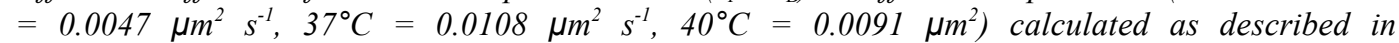
Supplementary Fig. 3a. $N=3\left(23.5^{\circ} \mathrm{C}, 40^{\circ} \mathrm{C}\right) . N=5\left(37^{\circ} \mathrm{C}\right)$. Boxplot displays the 25th and 75 th percentile, with the horizontal line at the median, squares represent the mean and whiskers $S D$. $\boldsymbol{b}$, Determination of STED-resolution by measuring the size of calibration beads on living neutrophils. The resolution depends clearly on the distance from the coverslip (depth). c, Confocal and STED images of living neutrophils during PMA-induced NET formation (z-direction: around 2-4 $\mu m$ from the cover slip; cell middle). Chromatin staining: SiR-DNA. Arrow = chromatin dots, mostly appearing close to the surface. Arrowhead = chromatin free areas occurring during the swelling process. Scale $=2 \mu \mathrm{m}$. Sometimes in these images small regions of higher intensity (small dots) were observed. It could be chromatin filled vesicles. d, Pressure curves measured by tipless cantilever AFM experiments. By getting in contact with a PMA activated cell (at least 90 minutes after activation, 100 nM PMA), the cell starts to push on the cantilever resulting in a deflection of the cantilever. Dividing these forces by the cellular contact area, an internal pressure was estimated (see Methods) that likewise increases until the membrane ruptures (end of the traces). Dashed lines are indicating the time points when the deflection values were reset manually to avoid signal loss of the AFM detector. $N=5$ donors $(n=14$ cells). 

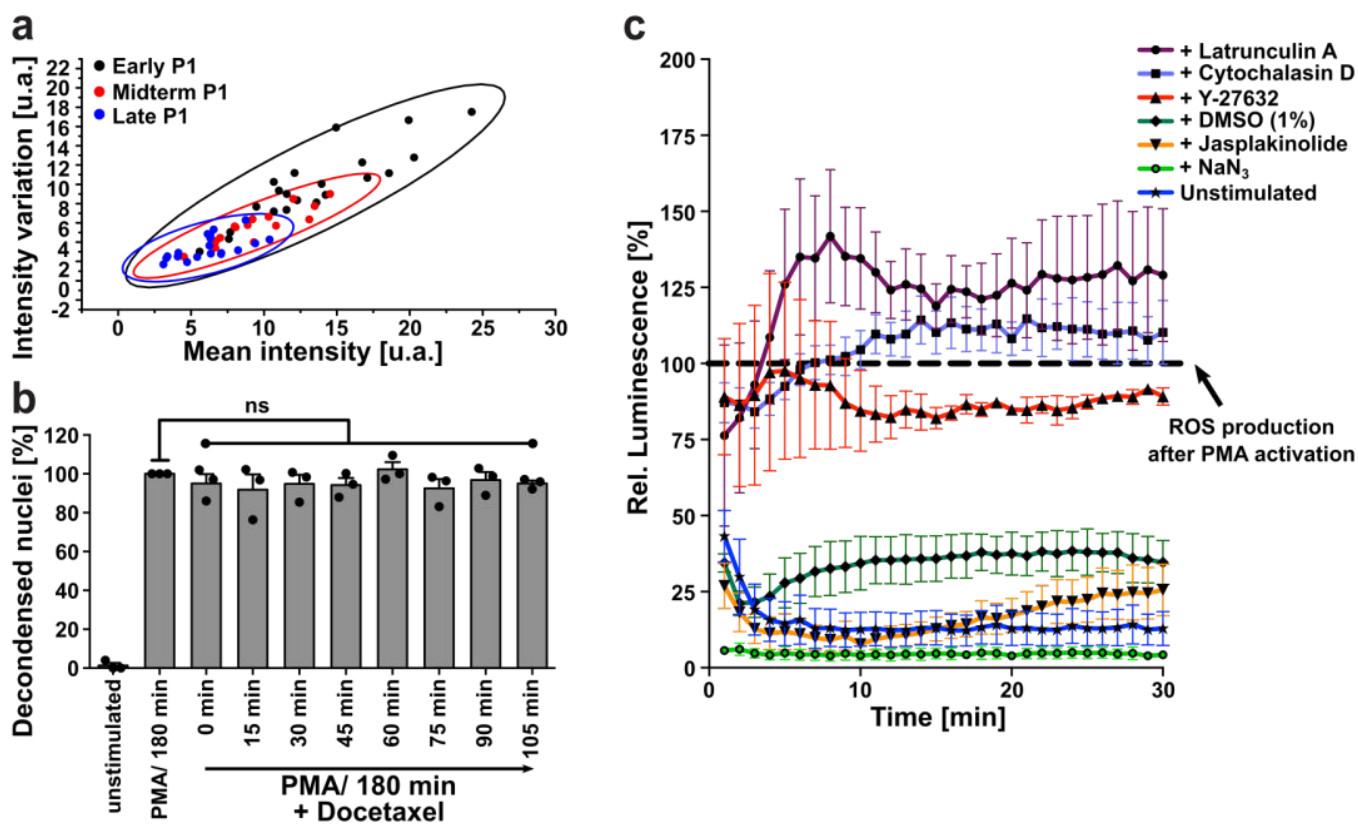

Supplementary Fig. 9: Influence of the cytoskeleton: a, Quantification of F-Actin disassembly. The mean fluorescence intensity of F-actin decreases with time during NETosis. At the same time the heterogeneity decreases, which is a measure for F-actin structure and not biased by bleaching. Thus, $F$-actin gets disassembled during NETosis. $N=1$ donor, data fitted with 95\% confidence ellipse. $\boldsymbol{b}$, NET formation after treatment with Docetaxel (100 $\mathrm{nM}$, inhibition of tubulin depolymerization) at different time points after induction of NETosis with PMA. Docetaxel shows no influence on NET formation (measured as \%-relative number decondensed nuclei after 180 min compared to exclusive activation with PMA). $n=3$. Mean \pm SEM. $n s=$ not significant. Statistics: One-way ANOVA (Bonferroni's multiple comparison test) c, Influence of actin cytoskeletal inhibition on PMA (100 nM)induced ROS production of human neutrophils determined by the chemiluminescence of luminol. Latrunculin A (violet) increases ROS, while Cytochalasin D (violet) and Y-27632 (red) have no or only slight effects on ROS production in the concentrations used for the experiments shown in Fig. $\mathbf{5 b}$, c. In contrast, Jasplakinolde (yellow) shows a strong inhibitory effect. As controls, the ROS levels of unstimulated cells (blue), cells after addition of 1\% DMSO (dark green, used for Jasplakinolide experiments) and $\mathrm{NaN}_{3}$ (green) are shown. $\mathrm{N}=3$. Mean \pm SEM. 


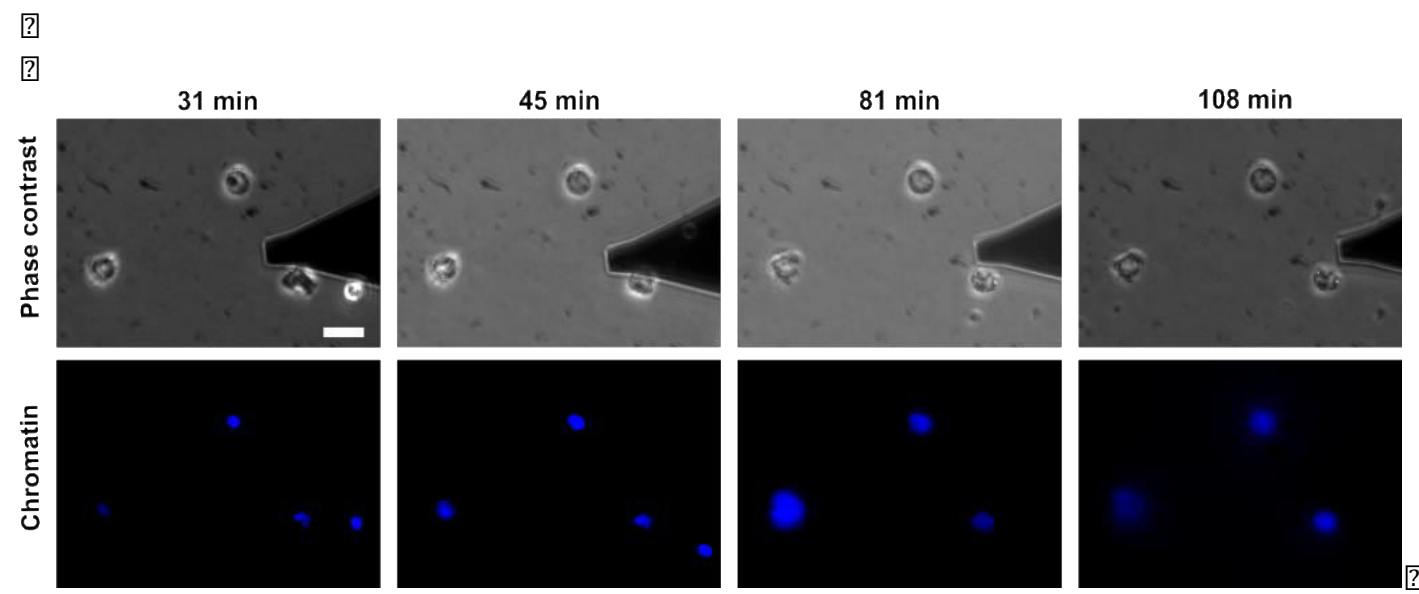

Supplementary Fig. 10: Morphology of neutrophils during AFM. Phase contrast and chromatin (blue) images of activated neutrophils during life cell AFM experiments. Within the early stage of NETosis, lobular shape of the nucleus and cell rounding can be observed. After around 40 minutes, $D N A$ decondenses und the cells rupture around $t=80-110 \mathrm{~min}$. No morphological difference between cells probed by AFM and control cells can be observed. Scale $=20 \mu \mathrm{m}$. 

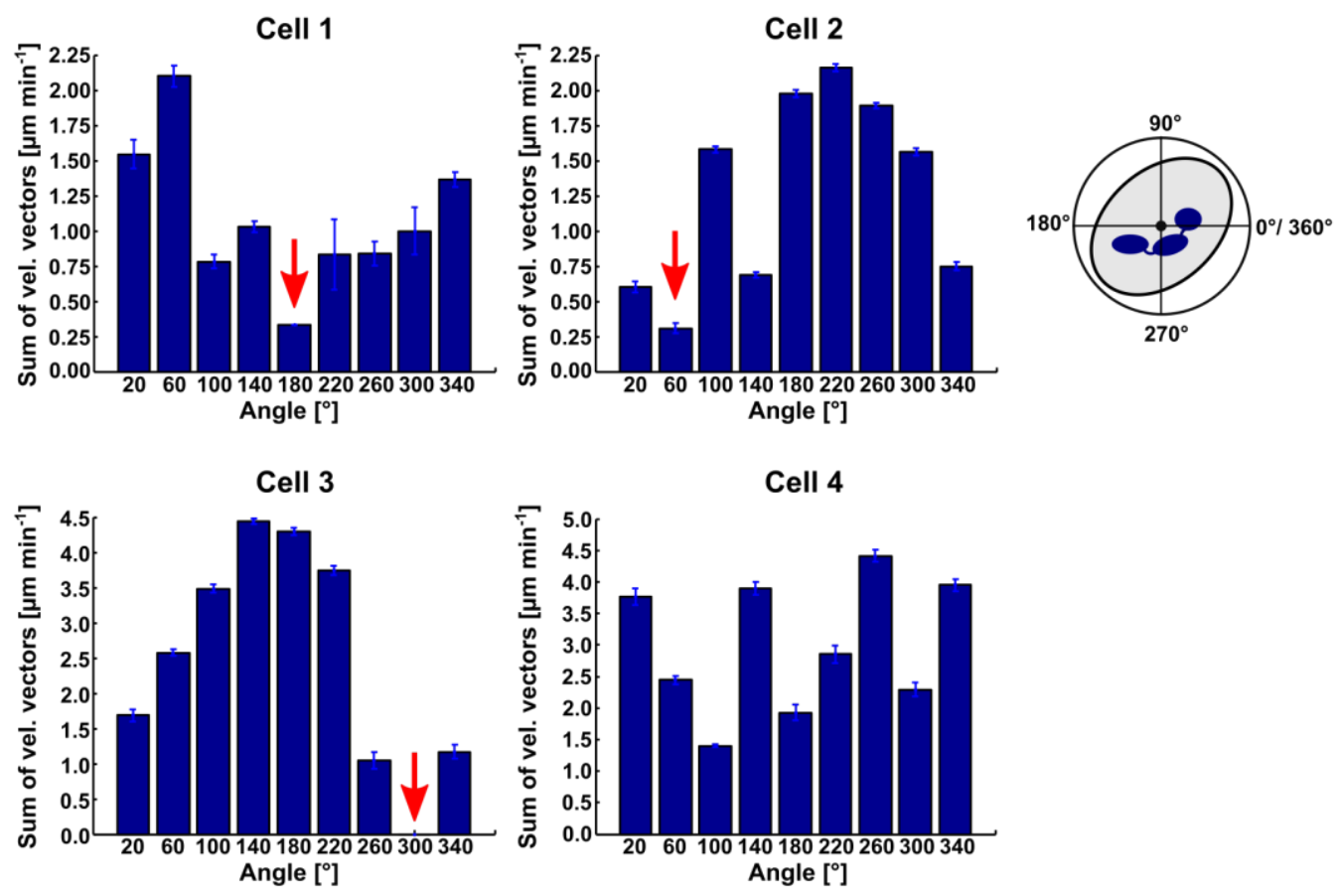

Supplementary Fig. 11: Chromatin swelling directs location of membrane rupture. Chromatin swelling analysis (data of the cells shown in Fig. 6a). To analyze whether the position of the rupture point correlates with the chromatin swelling behavior, the average swelling velocity and direction was calculated and visualized in a velocity plot (see also Methods). These velocity vectors were sorted (binning angle $=40^{\circ}$ ) and added up to find regions of different swelling behaviors. Regions of low velocities (red arrow, green circle in Fig. 6a) are often in close proximity to the observed rupture point position (red circle in Fig. 6a, cell 1-3), whereas no correlation is detectable for uniform swelling processes (cell 4). In this case, the nucleus is centered in the cell. Bar plot show the sum of all velocity vectors within one bin (vector length of all summed up velocity vectors). Error bars display SEM of the aforementioned vectors. 
a

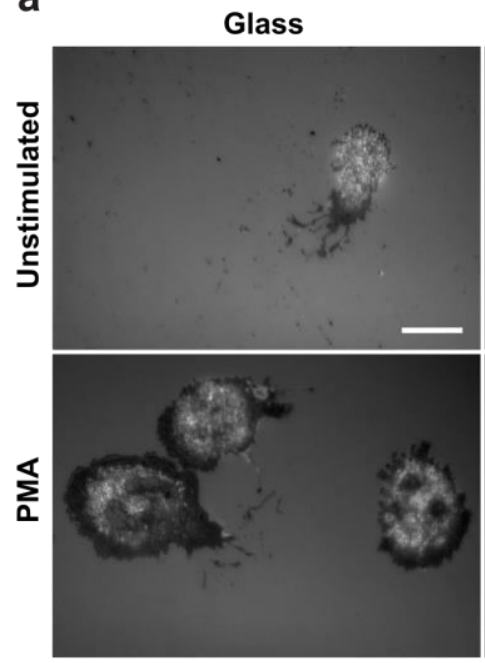

PLL

PLL-g-PEG

b
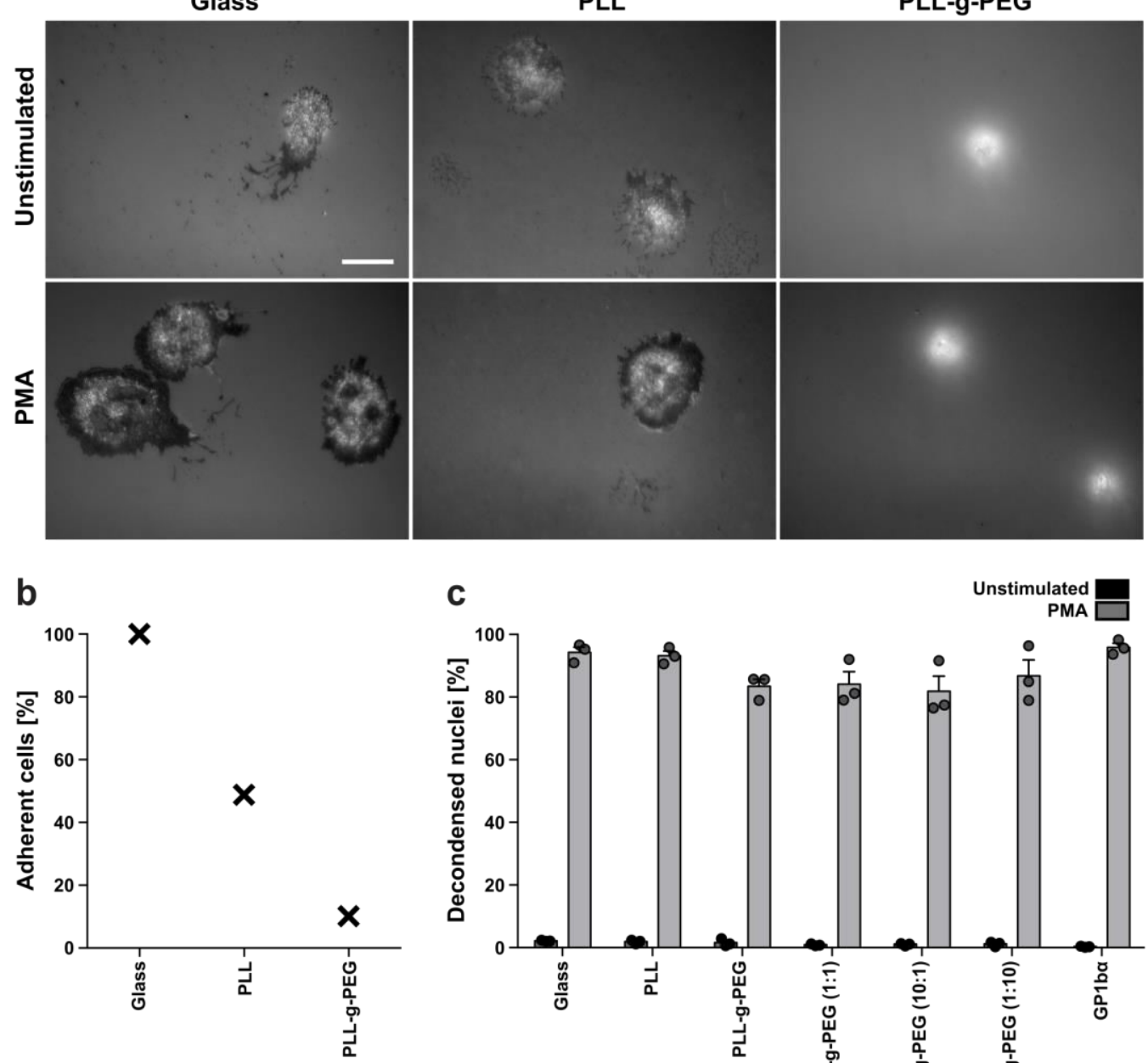

C

Unstimulated

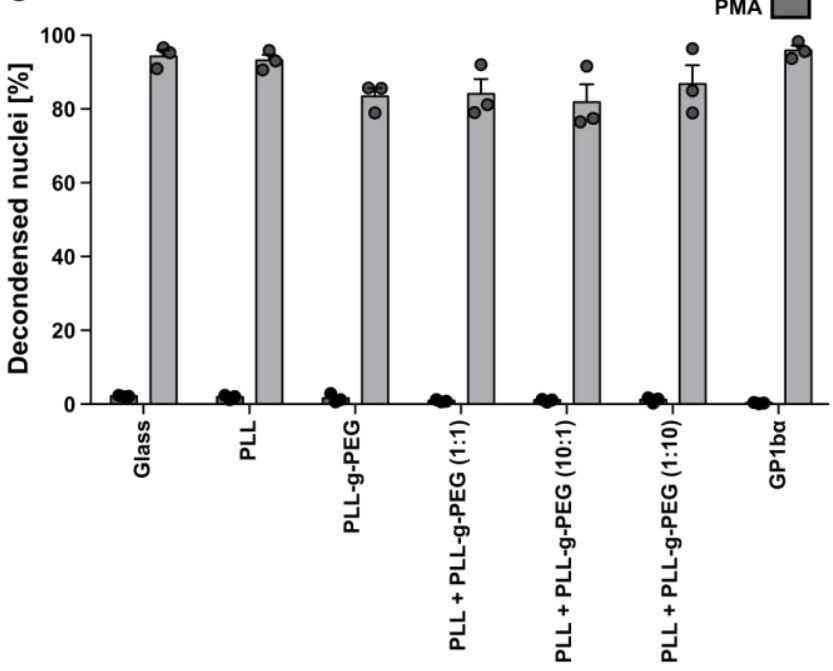

Supplementary Fig. 12: PMA-induced NETosis is independent of adhesion. a, RICM images of fixed neutrophils on glass after one-time washing. Images show cells incubated with PMA for 30 min or cells left unstimulated. Cells on PLL-g-PEG (Poly-L-Lysine-grafted-PolyEthylenGlycole) coated surfaces are barely adherent compared to neutrophils on glass or PLL coating. Scale $=10 \mu \mathrm{m}$. b, Cell numbers after one-time washing of cells seeded for $30 \mathrm{~min}$ on different surfaces. In PLL-g-PEG coated wells only a few cells remain, compared to glass and PLL coating. These cells are still barely adherent as shown in a. $N=1$. c, PMA-induced NETosis (100 nM) performed on different surfaces. The amount of decondensed nuclei is independent of the surface/adhesive properties (surfaces of different passivation level, GPIba is the ligand of Mac-1 integrin). No washing step was included in this procedure. $N=3$. Mean \pm SEM. 


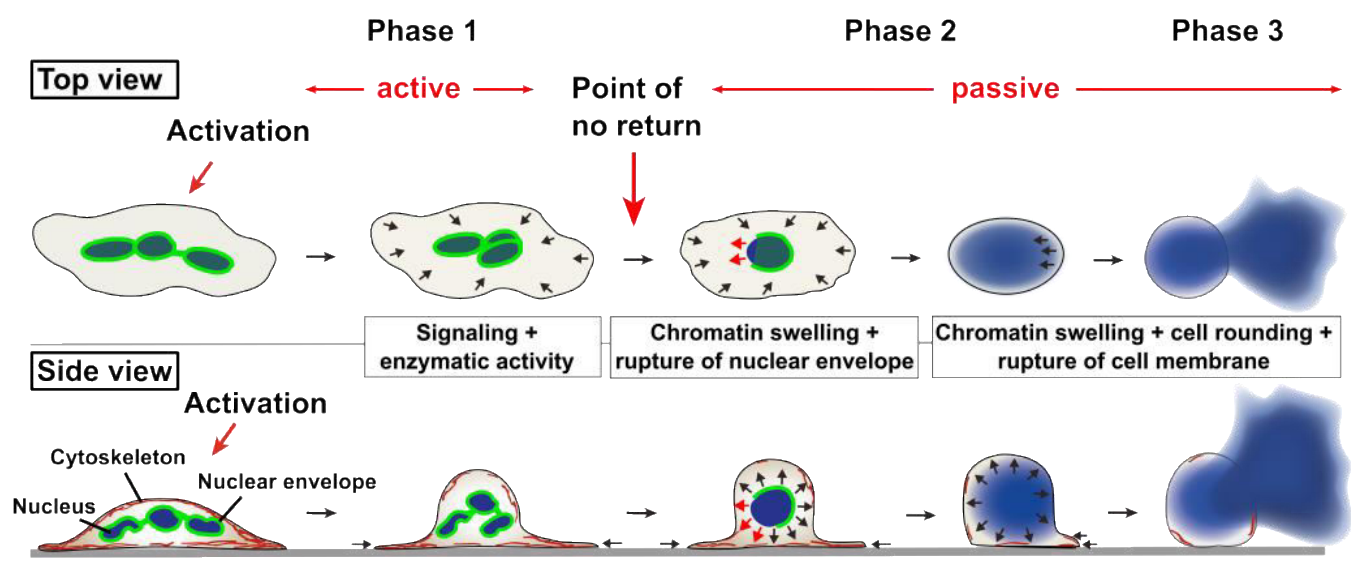

Supplementary Fig. 13: Detailed biophysical model of NET formation. [? 


\section{Supplementary Methods}

\section{Surface coating}

For platelet glycoprotein $1 \mathrm{~b}$ alpha chain (GP1b $\alpha$ ) coating, 96-glassbottom-well-plates were incubated over night with $5 \mu \mathrm{g} \mathrm{ml}^{-1} \mathrm{GP} 1 \mathrm{~b} \alpha$ (R\&G Systems) at $4^{\circ} \mathrm{C}$. Then, the wells were washed with $1 \mathrm{x}$ PBS followed by blocking with $3 \% \mathrm{BSA}$ (Roth) for $2 \mathrm{~h}$ at $37^{\circ} \mathrm{C}$ and an additionally washing step.

For Poly-L-lysine hydrobromide (PLL, Sigma-Aldrich) and poly(L-lysine)-graft-poly(ethylene glycol) (PLL-g-PEG, SuSoS AG) coating, 96-glassbottom-well-plates were incubated either with $0.5 \mathrm{mg} \mathrm{mL}^{-1}$ PLL in $10 \mathrm{nM}$ HEPES or $0.5 \mathrm{mg} \mathrm{mL}^{-1}$ PLL-g-PEG in $10 \mathrm{nM}$ HEPES at room temperature for $45 \mathrm{~min}$. Then solutions were removed and washed one time with culture medium. Further cell experiments were carried out as described below.

\section{Adhesion experiments}

Fresh isolated human neutrophils (10 000 per well) were seeded in 96-glassbottom-well-plates directly or wells coated with GP1b $\alpha$, PLL and/or PLL-g-PEG (0:1, 1:10, 1:1, 10:1, 1:0) as described above. Then, cells were activated for NETosis with PMA in a final concentration of $100 \mathrm{nM}$ for $3 \mathrm{~h}\left(37^{\circ} \mathrm{C}\right.$, $5 \% \mathrm{CO}_{2}$ ). All experiments were performed in triplicates. To end NET formation, cells were fixed with $2 \%$ PFA final concentration and stored over night at $4^{\circ} \mathrm{C}$. The fixed probes were stained with $1.62 \mu \mathrm{M}$ Hoechst 33342 and the percentage of decondensed nuclei/NETs was analyzed as described in the section Inhibitor Experiments in Methods.

To analyze the adhesion on the different surfaces, 10000 neutrophils per well were seeded in 96glassbottom well-plates coated with PLL or PLL-g-PEG or left untreated. The cells were washed one time with 1 XPBS after $30 \mathrm{~min}$ incubation $\left(37^{\circ} \mathrm{C}, 5 \% \mathrm{CO}_{2}\right)$ with or without PMA $(100 \mathrm{nM})$. Then, cells were fixed with $2 \%$ PFA final concentration. Subsequently, the cells were stained with Hoechst and the amount of remaining cells calculated relatively to the glass sample using ImageJ (six images/well counted in defined regions, counting blinded). Representative RICM images of the fixed samples were recorded in the setup described in the section Reflection Interference Contrast Microscopy (RICM) in Methods.

For time-lapse observations, 4 × $10^{5}$ cell per ml were seeded in an ibidi treat flow chamber coated with PLL-g-PEG and the movie recorded as described in the section Live Cell Imaging" in Methods.

\section{Reflection Interference Contrast Microscopy (RICM)}

For RICM, ibidi treated petri dishes (Ibidi $\mathrm{GmbH}$ ) with a $35 \mathrm{~mm}$ radius glass bottom (81158, ibidi) or ibidi treat flow chambers uncoated or coated with PLL were used. 100000 neutrophils per petri dish or $4 \times 10^{5}$ cells per $\mathrm{ml}$ per flow chamber were seeded and stained with Hoechst. The microscope setup contained a heating chamber (ibidi heating system, ibidi $\mathrm{GmbH}$ ) on top of the microscope stage (Axiovert 200, Zeiss) and the temperature was adjusted to $37 \mathrm{C}^{\circ}$ for all experiments. NETosis was induced by $100 \mathrm{nM}$ PMA. Time-lapse movies were recorded (1 frame per min) 63x magnified (EC Plan-Neofluar Ph3 objective/420481-9911-000, 1.6x Optovar, Zeiss) for both DAPI as well as RICM 
images. Samples were illuminated by a XCite Series 120Q or HBO 100 (1007-980, Zeiss) lamp together with a respective DAPI (Filter set 02 shift free/488002-9901-000, Zeiss) or RICM filter set (reflector module Pol ACR P\&Cfor HBO 100/424924-9901-000, emission filter 416 LP, AHF-Nr.: F76-416/000000-1370-927, Zeiss) and observed with a Zyla SCMOS camera (AndorZyla 5.5) using Micro Manager software (v.1.4) or a CoolSNAP ES camera (Photometrics) using the software Metamorph 6.3r2. (Molecular Devices Inc.). Subsequently, the image contrast was adjusted with ImageJ.

\section{ATP measurements/2-Deox-Gluc}

Fresh isolated human neutrophils (10 000 per well in RPMI (10 mM HEPES, 0.5\% HSA, without phenolred) were seeded in white 96-well-plates and metabolic activity inhibited by adding $5 \mathrm{mM} 2$ Deox-Gluc for defined time periods at $37^{\circ} \mathrm{C}(5 \mathrm{~min}, 15 \mathrm{~min}, 30 \mathrm{~min}, 60 \mathrm{~min}, 90 \mathrm{~min}$ and $120 \mathrm{~min}$ ). Simultaneously, cells were activated with PMA in a final concentration of $100 \mathrm{nM}$ or left untreated. After incubation the ATP amount was measured as described in the section ATP measurements in Methods. All experiments were carried out in triplicates and ATP levels were calculated relatively to the ATP amount of unstimulated cells incubated for $120 \mathrm{~min}$ without addition of 2-Deox-Gluc.

MPO activity

The activity of MPO was measured with the myeloperoxidase chlorination fluorometric assay kit from Cayman chemical following the company's instructions. To prove the inhibitory effect of $100 \mu \mathrm{M} 4$ ABAH on MPO, two different setups were used. First, the effect on purified MPO supplied by the company was studied in presents or absents of 4-ABAH. The activity of MPO was determined by the formation of fluorescein (Thermo Scientific APPLISKAN®, Software: Skanlt RE for Appliskan 2.3, Thermo Fisher Scientific) at $485 \mathrm{~nm} / 535 \mathrm{~nm}$ over $29.5 \mathrm{~min}$ (frame rate: $1.5 \mathrm{~min}$ ) at room temperature. All experiments were performed in triplicates. Second, human neutrophils (1 000000 per well in RPMI (10 mM HEPES, 0.5\% HSA)) were seeded in 24-well plates and activated with $100 \mathrm{nM}$ PMA in the presents or absents of $4-\mathrm{ABAH}$ for $15 \mathrm{~min}$ or $30 \mathrm{~min}$ at $37^{\circ} \mathrm{C}$, respectively. After incubation, the culture medium was removed and cells were washed with 1x PBS. Then, the cells were scraped from the plate and pelleted by $1000 \mathrm{~g}$ for $10 \mathrm{~min}$ at $4^{\circ} \mathrm{C}$. Cell lysis was induced by ultra shall sonication in combination with freeze-thraw cycles. After lysis, cell remnants were removed by centrifugation at 10 $000 \mathrm{~g}$ for $10 \mathrm{~min}$ at $4^{\circ} \mathrm{C}$ and MPO activity in the supernatant was determined as described above. For all conditions the change in fluorescence signal between $1 \mathrm{~min}$ and $29.5 \mathrm{~min}$ was determined and the relative MPO activity was calculated between 4-ABAH treated and untreated cells.

\section{Reactive oxygen species (ROS) measurement}

Fresh isolated human neutrophils (10 000 per well in HBSS (10 mM HEPES, without phenolred) were seeded in white 96 -well-plates (Greiner bio-one) at $37^{\circ} \mathrm{C}$. After seeding, luminol (Sigma-Aldrich) was added at a final concentration of $60 \mu \mathrm{M}$ and actin cytoskeletal components were inhibited by Cytochalasin D (100 nM), Latrunculin A $(1 \mu \mathrm{M})$, Jasplakinolide $(10 \mu \mathrm{M})$ or Y-27632 $(19.2 \mu \mathrm{M})$ 
respectively. Directly after addition of inhibitors, NETosis was activated with $100 \mathrm{nM}$ PMA and the luminescence was measured (GLOMAX ${ }^{\circledR} 96$ Microplate Luminometer, Software: GLOMAX 1.9.3, Turner BioSystems) at room temperature (frame rate: $1 \mathrm{~min}$ ). As controls the change in luminescence signal was recorded for unstimulated cells, cells treated with the maximal solvent concentration of $1 \%$ DMSO (used in Japlakinolide studies) and cells which were inhibited with $3 \mathrm{mM} \mathrm{NaN}$. All experiments were carried out in triplicates and the luminescence signal was determined relatively to ROS levels after exclusive PMA activation.

For evaluation of $\mathrm{NaN}_{3}$ activity, cells were seeded (90 000 per well) and activated as described above. $\mathrm{NaN}_{3}(3 \mathrm{mM})$ was added 5 min after activation with PMA and the change in the luminescence signal was continuously recorded.

\section{Cell toxicity}

Cell toxicity of cytoskeletal and metabolic inhibitors was measured by the release of lactatdehydrogenase (LDH) with the CytoTox 96® Non Radioactive Cytotoxicity Assay (Promega) as instructed by the company. By analogy with the assays described in the section Inhibitor Experiments in Methods, 10000 cells were seeded in plastic 96-well plates (Greiner bio-one) and incubated in RPMI (10 mM HEPES, 0.5\% HSA, without phenolred) with Cytochalasin D (100 nM), Latrunculin A $(1 \mu \mathrm{M})$, Docetaxel $(100 \mathrm{nM})$, Jasplakinolide $(10 \mu \mathrm{M})$, Y-27632 $(19.2 \mu \mathrm{M}), 2$ - Deox-Gluc $(5 \mathrm{mM})$, $\mathrm{NaN}_{3}(3 \mathrm{mM})$, 4-ABAH $(100 \mu \mathrm{M})$, DMSO $(1 \%)$ or only culture medium for 3 hours at $37^{\circ} \mathrm{C}$. The released LDH was measured in the supernatant as described in the assay protocol and cell toxicity was calculated relatively to maximal cell lysis.

Uptake of particles

Fresh isolated human neutrophils (100 000 per well in RPMI (10 mM HEPES, 0.5\% HSA) were seeded on pretreated (99\% alcohol) glass cover slips (\#1.5) in 24-well plates followed by preincubation for $30 \mathrm{~min}$ with or without 2- Deox-Gluc $(5 \mathrm{mM}), \mathrm{NaN}_{3}(3 \mathrm{mM})$ or 4 -ABAH $(100 \mu \mathrm{M})$, respectively. Then, cells were further incubated with fluorescein-labeled Escherichia coli (K-12 strain) BioParticles $^{\circledR}\left(0.1 \mathrm{mg} \mathrm{ml}^{-1}\right.$, Vybrant ${ }^{\mathrm{TM}}$ Phagocytosis Assay Kit (V-6694), Thermo Fisher Scientific) in presents of 2- Deox-Gluc ( $5 \mathrm{mM}), \mathrm{NaN}_{3}(3 \mathrm{mM})$ or 4-ABAH $(100 \mu \mathrm{M})$ and incubated for additionally $30 \mathrm{~min}$. For fixation, 2\% PFA final concentration was added and samples then stored after washing with 1x PBS. Cells were stained as described in the section Staining Procedure in Methods for F-actin and chromatin with Phalloidin (PromoFlpur-555P, PromoKine) and Hoechst directly after blocking. For each condition the particle uptake was analyzed manually by 3D confocal laser scanning microscopy (60x magnified, Olympus IX83 inverted microscope, software: Olympus Fluoview v.4.2) and the percentage of particle uptake calculated relatively to the total cell count of 60 cells per condition. 


\section{Quantification of fluorescence imaging}

For the quantification of Lamin B1 rupture, the rupture of the nuclear envelope was determined for different time points after induction of NETosis with PMA. Therefore, cells were stained for Lamin B1 and Chromatin as described in the section Staining Procedure in Methods and the nuclear envelope rupture was analyzed manually by 3D confocal laser scanning microscopy (60x magnified, Olympus IX83 inverted microscope, software: Olympus Fluoview v.4.2). For each condition the loss of the nuclear envelope (small rupture up to complete loss) was determined for 100 cells and calculated relatively to the total cell count.

Similarly, to quantify the loss of actin within phase 1, CLSM images of stained F-actin areas of fixed cells (exemplarily shown in Fig. 5a) where analyzed using the ImageJ thresholding plugin. More precisely, within all pictures, the F-actin area of individual cells were segmented first (Yen thresholding method) and the intensity values of the enclosed actin area was averaged to obtain both, a mean intensity value as well as a number for the intensity variation within the respective area (standard deviation). Both values were plotted to visualize the correlation between the amount and the homogeneity of actin within P1 and a 95\% confidence ellipse was drawn. 


\subsection{Manuscript 2}

\section{Kinetic Requirements for Spatiotemporal Chemical Im- aging with Fluorescent Nanosensors}

\section{ACS Nano}

Published: 5 April 2017

Daniel Meyer ${ }^{1,2}$, Annika Hagemann ${ }^{1,2} \&$ Sebastian Kruss ${ }^{1,2}$

${ }^{1}$ Institute of Physical Chemistry, Göttingen University, Göttingen 37077, Germany

${ }^{2}$ Center of Nanoscale Microscopy and Molecular Physiology of the Brain (CNMPB), Göttingen 37073, Germany 


\title{
Kinetic Requirements for Spatiotemporal Chemical Imaging with Fluorescent Nanosensors
}

\author{
Daniel Meyer, ${ }^{\dagger, \ddagger}$ Annika Hagemann, ${ }^{\dagger \neq}$ and Sebastian Kruss ${ }^{*}, \dagger, \neq(\mathbb{0}$ \\ ${ }^{\dagger}$ Institute of Physical Chemistry, Göttingen University, Tammannstrasse 6, Goettingen 37077, Germany \\ ${ }^{\ddagger}$ Center for Nanoscale Microscopy and Molecular Physiology of the Brain (CNMPB), Göttingen 37073, Germany \\ S Supporting Information
}

\begin{abstract}
Fluorescent nanosensors are powerful tools for basic research and bioanalytical applications. Individual nanosensors are able to detect single molecules, while ensembles of nanosensors can be used to measure the bulk concentration of an analyte. Collective imaging of multiple nanosensors could provide both spatial and temporal chemical information from the nano- to the microscale. This type of chemical imaging with nanosensors would be very attractive to study processes such as chemical signaling between cells (e.g., neurons). So far, it is not understood what processes are resolvable (concentration, time, space)

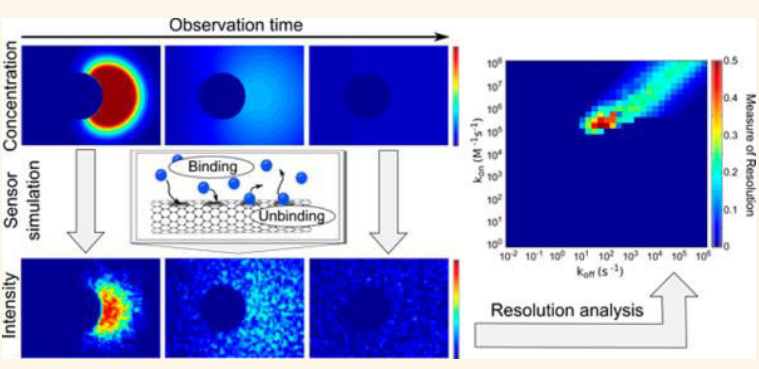
and how optimal sensors should be designed. Here, we develop a theoretical framework to simulate the fluorescence image of arrays of nanosensors in response to a concentration gradient. For that purpose, binding and unbinding of the analyte is simulated for each single nanosensor by using a Monte Carlo simulation and varying rate constants $\left(k_{\text {on }}, k_{\text {off }}\right)$. Multiple nanosensors are arranged on a surface and exposed to a concentration pattern $c_{A}(x, y, t)$ of an analyte. We account for the resolution limit of light microscopy (Abbe limit) and the acquisition speed and resolution of optical setups and determine the resulting response images $\Delta I(x, y, t)$. Consequently, we introduce terms for the spatial and temporal resolution and simulate phase diagrams for different rate constants that allow us to predict how a sensor should be designed to provide a desired spatial and temporal resolution. Our results show, for example, that imaging of neurotransmitter release requires rate constants of $k_{\text {on }}=10^{6} \mathrm{M}^{-1} \mathrm{~s}^{-1}$ and $k_{\text {off }}=10^{2} \mathrm{~s}^{-1}$ in many scenarios, which corresponds to high dissociation constants of $K_{\mathrm{d}}>100 \mu \mathrm{M}$. This work predicts if a given fluorescent nanosensor array (rate constants, size, shape, geometry, density) is able to resolve fast concentration changes such as neurotransmitter release from cells. Additionally, we provide rational design principles to engineer nanosensors for chemical imaging.
\end{abstract}

KEYWORDS: chemical imaging, single-molecule methods, biosensors, fluorescence microscopy, kinetics, simulations, signaling

hemical imaging is a powerful concept. It provides spatially resolved chemical information about a sample. For example, spectroscopic methods can be used to collect spectra of a sample from different locations. In the case of Raman spectroscopy, these spectra contain information about the vibrational states of the sample. ${ }^{1,2}$ Another example for chemical imaging is fluorescence lifetime imaging (FLIM). ${ }^{3}$ It provides information about the fluorescence lifetime and quantum yield of the sample, which is related to the chemical environment and the presence of quenching species. In general, chemical imaging only requires spatially resolved chemical information such as spectra or the concentration of a certain molecule. $^{4}$

In contrast to chemical imaging, many methods such as fluorescence microscopy rely on labeling structures with artificial fluorophores. The resulting image provides informa- tion about the spatial and temporal distribution of the labeled structure. This approach has been extremely successful in elucidating the (static) structure of cells. ${ }^{5}$ However, the label itself does not necessarily provide information about the chemical environment around the fluorescent label. It mainly shows where the label is.

Alternatively, fluorescent probes/sensors are ideal candidates for chemical imaging. ${ }^{6,7}$ They are designed in such a way that they change their fluorescence properties in the presence of a specific molecule. Therefore, they report the local concentration of this molecule up to single binding events. ${ }^{8}$ One example of chemical imaging with fluorescent sensors is

Received: January 25, 2017

Accepted: April 5, 2017

Published: April 5, 2017 
a

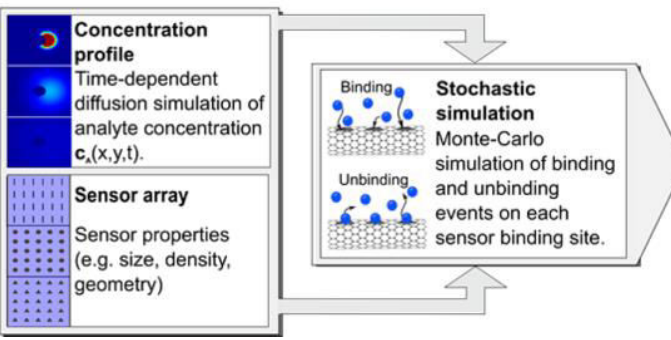

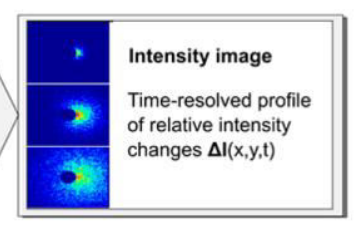

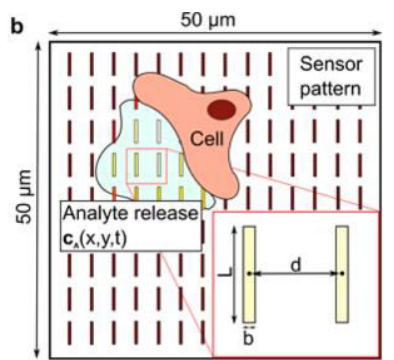

Figure 1. Simulating the response image of a fluorescent nanosensor array exposed to an analyte. (a) Flow diagram of the simulation: (1) Concentration profile $c_{A}(x, y, t)$ of analyte $A$ is simulated. Here, neurotransmitter release from a neuron was simulated. (2) Fluorescent nanosensors of variable size and geometry are placed in positions $\left(x_{\mathrm{S}}, y_{\mathrm{S}}\right)$ to create a sensor array and are exposed to the concentration profile $c_{\mathrm{A}}(x, y, t)$. (3) Binding and unbinding of the analyte to every single nanosensor in position $\left(x_{\mathrm{S}}, y_{\mathrm{S}}\right)$ is simulated using a stochastic Monte Carlo simulation and rate constants $k_{\text {on }}$ and $k_{\text {off. }}$ If an analyte binds or unbinds it changes the fluorescence of the sensor and consequently the signal of a single sensor $\Delta I$ at time point $t$ can be calculated. (4) Finally, the image series $\Delta I(x, y, t)$ is calculated by overlaying the fluorescence intensity point spread functions of all sensors and accounting for the resolution limit of light microscopy (Abbe limit), pixel size, and frame rate of the detector. These time-resolved images are used to study the spatial and temporal resolution of the nanosensor array for different parameters and scenarios. (b) Sensor array (not to scale) with sensors of anisotropic shape such as nanotubes $(50 \times 50 \mu \mathrm{m}$ area, distance between sensors $d=500 \mathrm{~nm}$, sensor size $1 \times 300 \mathrm{~nm}$ ). A cell on top releases a molecule of interest exposing the array to a certain concentration profile $c_{\mathrm{A}}(x, y, t)$.

oxygen-sensitive fluorescent paints that are used to image the aerodynamic profile of cars and airplane wings. ${ }^{9,10}$ Chemical imaging is also feasible for biomedical purposes. For example, $\mathrm{pH}$-sensitive dyes have been used to image in vivo the $\mathrm{pH}$ of skin wounds during healing. ${ }^{11}$ Another example is calciumsensitive dyes or proteins. Time-resolved images of these fluorophores are useful in understanding intracellular signaling and signal propagation in neuronal circuits. ${ }^{12}$ The success of these dyes underlines that there is a large need for chemical imaging of other molecules. Fluorescent sensors that are able to detect neurotransmitters could be valuable tools to elucidate the chemical connectome of neural circuits. ${ }^{13-16}$ Genetically encoded fluorescent proteins are promising candidates to detect neurotransmitters, but they are limited to the cell surface and have drawbacks such as bleaching. ${ }^{17}$

To circumvent bleaching and gain other beneficial photophysical properties nanomaterials can be used as building blocks for sensors/probes. ${ }^{7,18}$ Nanosensors are able to detect single molecules and have been demonstrated for different classes of molecules. ${ }^{7,19-21}$ They are typically composed of a fluorescent nanomaterial and a recognition unit that binds the analyte and transduces the binding event. Single-walled carbon nanotubes (SWCNTs) are a new class of fluorescent building blocks for these nanosensors. ${ }^{21}$ They have useful properties such as ultralow photobleaching, large Stokes shifts, and fluorescence in the tissue transparent near-infrared (nIR) window. $^{19,21}$ SWCNTs can be functionalized to render their fluorescence sensitive to different biomolecules including neurotransmitters, sugars, proteins, and explosives. ${ }^{19,22-29}$ The mechanism of fluorescence modulation is still under debate and differs for different functionalization strategies and analytes. $21,24,26,30-32$

As outlined above, the idea of chemical imaging with fluorescent paints or coatings has been demonstrated mainly for organic dyes and small molecules such as oxygen. ${ }^{33}$ Nanomaterial-based sensors are much less explored in this context even though they promise advantages such as ultralow photobleaching. One example for nanosensor-based chemical imaging are modified terbium nanoparticles, which were used to measure physical quantities such as temperature. ${ }^{34}$ Recently, $\mathrm{pH}$-sensitive upconverting nanoparticles have been used to study the trafficking of vesicles and their corresponding $\mathrm{pH}$ in a cell model. ${ }^{35}$ Immobilized (single) nanosensors have been used to detect efflux of molecules from cells, but the sensors were individually analyzed and not as a collective chemical image. ${ }^{29,36}$ For example, hot spots of dopamine release from neuroprogenitor cells were identified by analyzing the fluorescence response of carbon nanotube based sensors along the cell contour. ${ }^{29}$ In this case, the individual responses were analyzed and not the response of many sensors together (i.e., a chemical image). So far, it is not understood how the collective chemical image of many sensors reflects the concentration of an analyte.

In order to describe the binding state of a nanosensor, deterministic kinetics are not appropriate anymore, and it becomes necessary to switch to stochastic kinetics. In the past, algorithms such as the Gillespie algorithm have been developed to describe chemical reactions. ${ }^{37}$ For surface-bound sensors, the description can become more complex when convection or flow plays a role. ${ }^{38}$

To our knowledge, so far, no one has combined (1) a stochastic kinetic description of nanosensors immobilized on a surface that is (2) exposed to a changing analyte concentration to (3) simulate fluorescence response images and how they change over time (Figure 1).

In this work, we develop a theoretical framework for chemical imaging with nanosensors. The basic idea is to combine the beneficial properties of nanosensors but image not only one but many to gain chemical images over large microscale areas with nanoscale resolution. Our work is motivated by carbon nanotube based fluorescent sensors, but every other type of (nano)sensor is covered by this work as well. As concentration profiles we simulate the release of neurotransmitters from cells because signaling between neurons is one of those biological problems that could benefit most from chemical imaging. In our model, we describe the single nanosensors using a stochastic Monte Carlo simulation and place them in arrays on a surface. We then expose them to a simulated concentration gradient (in space and time) of a molecule of interest to simulate binding/unbinding events and the fluorescence change $\Delta \mathrm{I}_{s}(x, y, t)$. Finally, the image of many sensors is calculated by accounting for the resolution limit of 


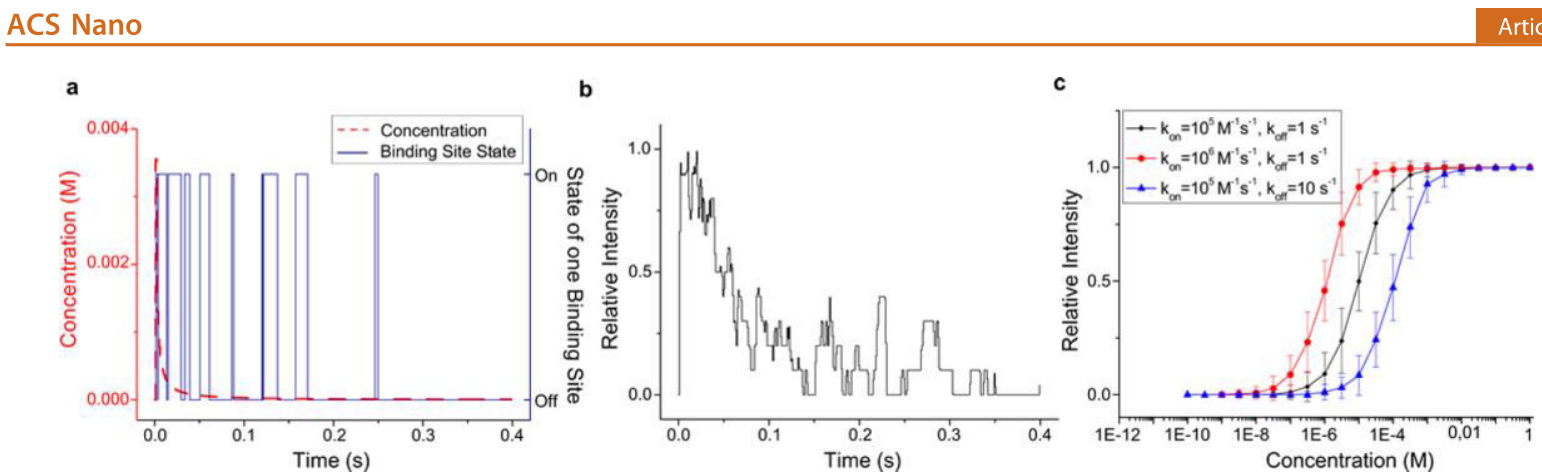

Figure 2. Stochastic kinetic simulation of single nanosensors. (a) (Red) Time-dependent concentration profile close to a cell after release of a signaling molecule (e.g., a neurotransmitter). (Blue) Simulated states of a single binding site for this concentration profile and the reaction rate constants $k_{\mathrm{on}}=10^{6} \mathrm{M}^{-1} \mathrm{~s}^{-1}$ and $k_{\text {off }}=10^{2} \mathrm{~s}^{-1}$. (b) Intensity response of a single sensor with $n=10$ binding sites for the concentration profile shown in a. Steps can be smaller than maximum response/number of binding sites because the simulation calculates the mean binding state for every simulation run $(1 \mathrm{~ms})$. (c) Ensemble responses (calibration curves) for different rate constants. A single sensor was exposed to an analyte with a concentration between $0.1 \mathrm{nM}$ and $1 \mathrm{M}(N=100$ simulation runs for $t=10 \mathrm{~s}$ to obtain the mean static response). Error bars are standard deviations.

light microscopy and other restrictions such as frame rates. This simulation is then used to explore how different parameters such as rate constants affect images. Expressions for spatial, temporal, and concentration resolutions are derived and summarized in phase diagrams. These results can serve as a guideline for sensor development and are useful in understanding and interpreting experimental images obtained from nanosensor arrays.

\section{RESULTS AND DISCUSSION}

Stochastic Kinetic Description of Single Sensors. The first step toward simulating the image of multiple fluorescent sensors is to describe the fluorescence intensity of every single sensor (see the Materials and Methods for details). We developed a modified stochastic kinetic Monte Carlo simulation (Gillespie algorithm) to calculate the fluorescence intensity of single nanosensors. ${ }^{37,39}$ We assumed that each sensor $S$ has $n$ binding sites for a molecule of interest, e.g., a signaling molecule A. Therefore, the bimolecular reaction equation is described by

$$
\mathrm{S}+\mathrm{A} \underset{k_{\text {off }}}{\rightleftharpoons} \mathrm{SA}
$$

If sensors are immobilized, their (local) concentration is constant. Then the time $\tau_{\text {on }}$ that a single neurotransmitter needs to bind to the $j$ th binding site can be described by

$$
\tau_{\text {on }}=\frac{1}{c_{\mathrm{A}}\left(x_{s}, y_{s}, t\right) k} \ln \left(\frac{1}{r_{\text {on }}}\right)
$$

Here, $c_{\mathrm{A}}\left(x_{\mathrm{S}}, y_{\mathrm{S}}, t\right)$ is the concentration of the analyte in location $\left(x_{\mathrm{S}}, y_{\mathrm{S}}\right)$ at time $t, k_{\mathrm{on}}$ the corresponding forward (binding) rate constant, and $r_{\text {on }}$ a random number between 0 and 1 . Rate constants can be experimentally measured by performing single nanosensor experiments and employing models to extract them (Hidden Markov models, birth-and-death models, etc.). ${ }^{40}$ For an existing nanosensor, one could employ these approaches to extract rate constants and use them in our simulation to predict its performance for chemical imaging. The time $\tau_{\text {off }}$ for unbinding does not depend on the analyte concentration anymore and is described by

$$
\tau_{\text {off }}=\frac{1}{k_{\text {off }}} \ln \left(\frac{1}{r_{\text {off }}}\right)
$$

Here, $k_{\text {off }}$ is the backward (unbinding) rate constant and $r_{\text {off }}$ a random number between 0 and 1 . In most scenarios, we assumed that our sensors can bind up to $n=10$ analytes and that there is no cooperativity (see below and Figure S1).

Consequently, we can use this framework for a stochastic simulation algorithm (SSA) that simulates binding/unbinding events at each binding site of a sensor $S$ within a given period of time $\Delta t$. This algorithm was iterated for every sensor and binding site (see the Materials and Methods for details).

It returns a temporal-state distribution $B_{j}(t)$ of each binding site within the time interval $[t ; t+\Delta t]$. The addition of all distributions within a respective sensor $S$ leads then to the number of "bound" sensor binding sites, which is averaged over all time steps (that can be $<\Delta t$ ) and normalized to generate the relative change (increase for our model system) of sensor intensity $\Delta I_{S}(\mathrm{~S}, t)$ :

$$
\Delta I_{S}(S, t)=\frac{1}{n} \sum_{j=1}^{n} \frac{1}{\Delta t} \int_{t}^{t+\Delta t} B_{j}\left(t^{\prime}\right) \mathrm{d} t^{\prime}
$$

This algorithm allows us to simulate the intensity of a sensor for a given time-dependent concentration profile. Figure 2a shows a typical concentration profile that can be expected from a single release from a cell. The concentration starts in the millimolar range and decays within milliseconds. The status of a binding site can be bound (1) or not bound (0). Figure 2a shows the time-course of a binding site for this concentration profile and reveals the intrinsic stochasticity of the process. When the number of binding sites is increased to $n=10$ (Figure 2b), the response becomes less discrete than for $n=1$ and the trace of the on/off states in Figure 2a. Simulations with different numbers of binding sites show that with more than $n>$ 4 binding sites single sensor responses become less stochastic and decrease to around $2 / 3$ of the maximal stochastic fluctuations (Figure S1b). We chose $n=10$ binding sites because our model sensor system contains carbon nanotubebased sensors. They most likely have multiple binding/ adsorption sites. For example, protons quench carbon nanotube fluorescence in steps, which can be explained by an exciton 

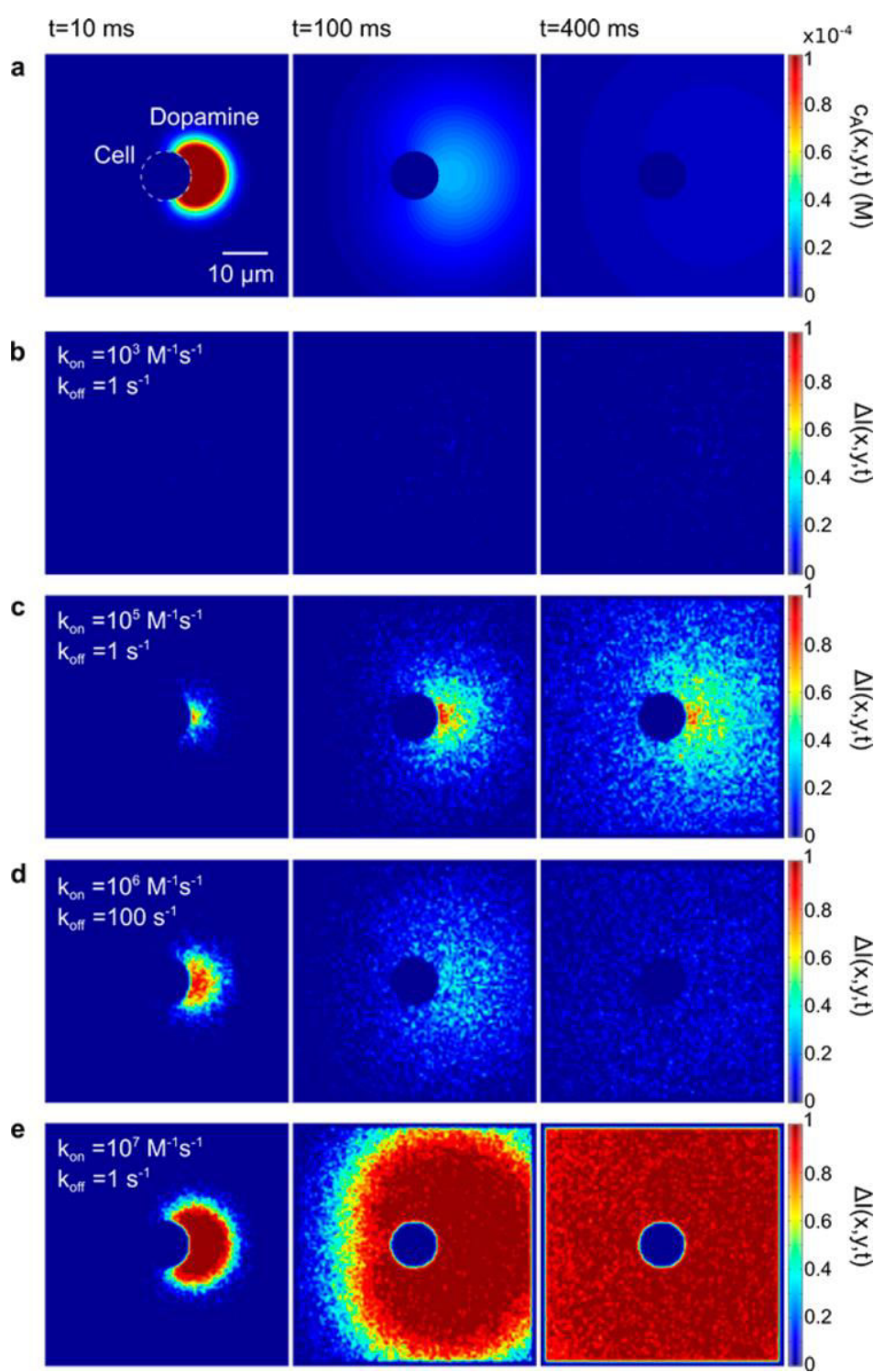

Figure 3. Image of a nanosensor array in response to release of molecules from a cell. (a) Time-dependent concentration profile. Release and diffusion of the neurotransmitter dopamine after exocytosis from a single vesicle fusion on a single cell $(d=10 \mu \mathrm{m})$ was simulated and yields a concentration profile $c_{\mathrm{A}}(x, y, t)$ at different time points $t$. $(\mathrm{b}-\mathrm{e})$ Response images for different forward and backward rate constants $k_{\text {on }}$ and $k_{\text {off }}$ The simulated response intensity images $\Delta I(x, y, t)$ show the strong influence of rate constants on spatial and temporal resolution. $k_{\text {on }}=10^{3}$ $\mathrm{M}^{-1} \mathrm{~s}^{-1}, k_{\mathrm{off}}=1 \mathrm{~s}^{-1}$ leads to almost no sensor response (b). $k_{\mathrm{on}}=10^{5} \mathrm{M}^{-1} \mathrm{~s}^{-1}, k_{\mathrm{off}}=1 \mathrm{~s}^{-1}$ causes a response image similar to the concentration profile $\mathrm{c}_{\mathrm{A}}(x, y, t)$ but does not resolve the concentration decay $(\mathrm{c})$. The case $k_{\text {on }}=10^{6} \mathrm{M}^{-1} \mathrm{~s}^{-1}, k_{\text {off }}=10^{2} \mathrm{~s}^{-1}$ resolves the concentration profile and the concentration decay due to faster $k_{\text {off }}(\mathrm{d}) . k_{\mathrm{on}}=10^{7} \mathrm{M}^{-1} \mathrm{~s}^{-1}, k_{\text {off }}=1 \mathrm{~s}^{-1}$ causes a faster saturation (e) and shows that sensors with low dissociation constant $K_{\mathrm{d}}=k_{\text {off }} / k_{\text {on }}$ are not necessarily the best choice to image fast chemical signaling.

diffusion length smaller than the nanotube length and suggesting multiple binding sites. ${ }^{41}$

Furthermore, if one assumes that the functionalization with macromolecules like DNA is periodic the pitch size is on the order of tens of nanometers and consequently the number of repeating chemical motifs is on the order of ten for these sensors. ${ }^{30}$ Other numbers of binding sites can be tested in the simulation algorithm as well.

In our model we assumed that the binding sites do not affect each other (no cooperativity). It was shown that excitons explore the chemical environment of carbon nanotube-based sensors. If the exciton hits for example a quenching site caused by an adsorbed analyte it is quenched. ${ }^{41}$ In this picture the exciton is either quenched or not and consequently the fluorescence changes are of similar size. Furthermore, because it is either quenching or not the exciton has no memory, whether it already passed an adsorbed analyte. Therefore, at least for this type of mechanism the assumption of no cooperativity is justified.

The rate constants are the most important parameters of these sensors and determine how long it takes until an 

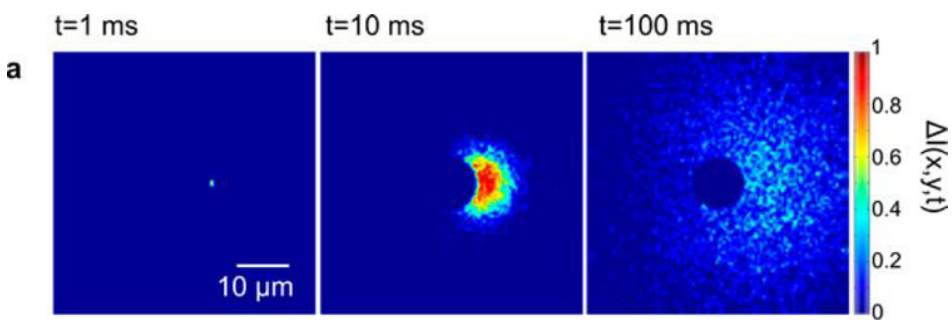

b
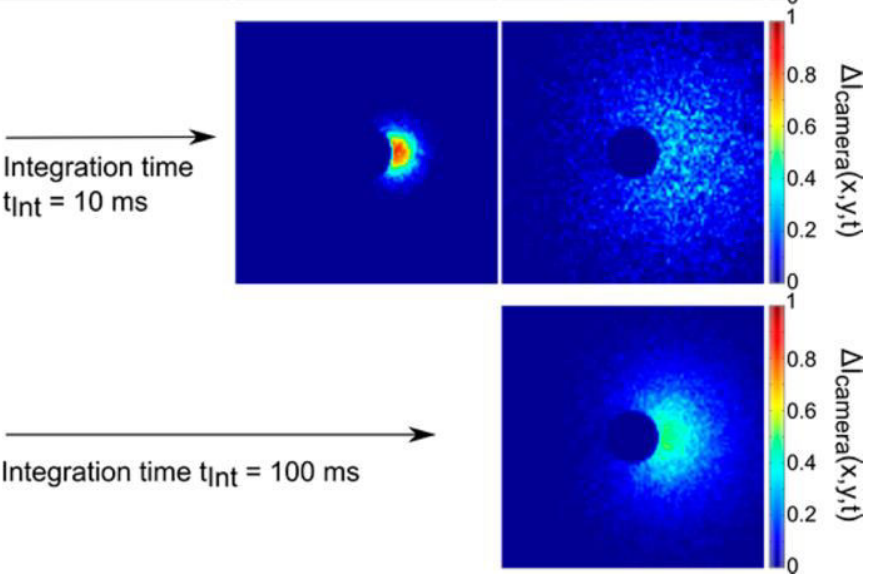

Figure 4. Impact of detector frame rate on resolution. (a) Frame rate/integration time of the detector (camera) strongly affects the images that can be recorded for a given sensor array and concentration profile. Here, the sensor array is exposed to the concentration profile $c_{A}(x, y, t)$ from Figure 3. For $t_{\mathrm{int}}=1 \mathrm{~ms}$, the release point and later the concentration gradient is resolved (a). (b, c) When the integration time is increased to 10 or $100 \mathrm{~ms}$ not only the temporal but also the spatial resolution decreases $(\mathrm{b}, \mathrm{c})$. Here, $k_{\mathrm{on}}=10^{6} \mathrm{M}^{-1} \mathrm{~s}^{-1}, k_{\mathrm{off}}=10^{2} \mathrm{~s}^{-1}$ was used for the simulation.

unbound state is reached after the concentration becomes very low.

Calibration curves can be generated by simulating the response of a single nanosensor multiple times at a static concentration of analyte. These curves represent the ensemble response of these sensors (Figure 2c). Again, the rate constants determine the position of the calibration curves, which are centered around the dissociation constants $K_{\mathrm{d}}=k_{\text {off }} / k_{\text {on }}$.

Overlay of Single Sensors. In the next step, multiple sensors were investigated similar to a paint composed of individual nanosensors. For that purpose, we used arrays of sensors of certain geometry, size, and density. Every single fluorescent sensor creates a point spread function (PSF), and therefore, the collective image of many sensors is an overlay of all these PSFs, which therefore accounts for the resolution limit of light microscopy (Abbe limit). The overlay was implemented by overlaying airy disks of every sensor (see the Materials and Methods for details).

This step is conceptionally important because it is the step from the description of a single sensor to an image of sensors that can be collected in a microscope. For most simulations, we assumed that our sensors are rodlike objects with a size of $1 \times$ $300 \mathrm{~nm}^{2}$ and a distance $d=500 \mathrm{~nm}$ to the center of its next neighbors, resulting in a symmetrical sensor pattern.

This geometry is motivated by previous work on carbon nanotube-based sensors that can serve as a model system for this concept of chemical imaging with nanosensors. ${ }^{19-22} \mathrm{We}$ will show later that size, shape, and arrangement of sensors does not play an important role as long as distances between individual sensors are below the diffraction limit and diffusion of analytes is fast as expected from most relevant biological systems.

The result of this operation is an image $\Delta I_{\mathrm{A}}(x, y, t)$ that can be simulated with mesh-sizes (pixel sizes) that are only limited by computational costs. Typically, we used a resolution of $100 \mathrm{~nm}$. However, in a microscope this image would be convoluted by the camera acquisition and pixel size. The acquisition speed of the camera is often not limited by the frame rate of the camera but rather the photon flux from the sample.

The transformation of the concentration profile to an image can be described by a convolution of several functions. $f$ : $c_{\mathrm{A}}(x, y, t) \rightarrow \Delta I_{\mathrm{S}}(x, y, t)$ describes the response of single sensors to the concentration gradient. Next, g: $\Delta I_{S}(x, y, t) \rightarrow \Delta I_{\mathrm{A}}(x, y, t)$ uses points spread functions to account for the Abbe limit. Finally, h: $\Delta I_{\mathrm{A}}(x, y, t) \rightarrow \Delta I(x, y, t)$ accounts for the temporal and spatial resolution of the camera and optical system. The final response image $\Delta I(x, y, t)=(\mathrm{h} \rightarrow \mathrm{g} \rightarrow \mathrm{f})\left(c_{\mathrm{A}}(x, y, t)\right)$ is what can be recorded in a real microscope for specific parameters including size, rate constants, etc. In turn, the inverse function (if it exists) can be used to receive the concentration profile from experimental data $c_{\mathrm{A}}(x, y, t)=\left(\mathrm{f}^{-1} \rightarrow \mathrm{g}^{-1} \rightarrow \mathrm{h}^{-1}\right)$. $(\Delta I(x, y, t))$. Typical examples of those images are shown in Figure 3. In Figure $3 \mathrm{a}$, a simulated concentration profile of dopamine release is shown (simulated with COMSOL 4.1).

The cell (10 $\mu \mathrm{m}$ diameter) was modeled in $2 \mathrm{D}$ as nondiffusive material and placed in the middle of a $50 \times 50$ $\mu \mathrm{m}$ water-filled box. To verify that these $2 \mathrm{D}$ simulations do not lead to wrong conclusions, we also simulated and compared concentration profiles in 3D (see below and Figure S2). Vesicles had a diameter of $100 \mathrm{~nm}$ and were placed on the edge of the cell and filled with the neurotransmitters dopamine $\left(c_{0}=\right.$ 


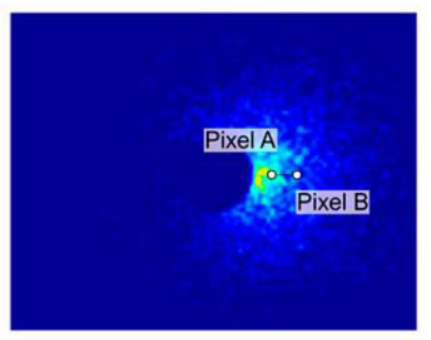

b

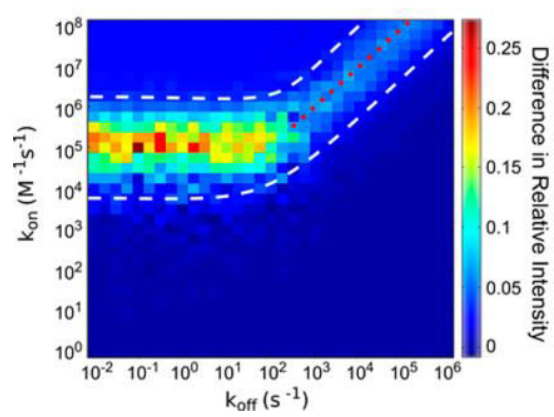

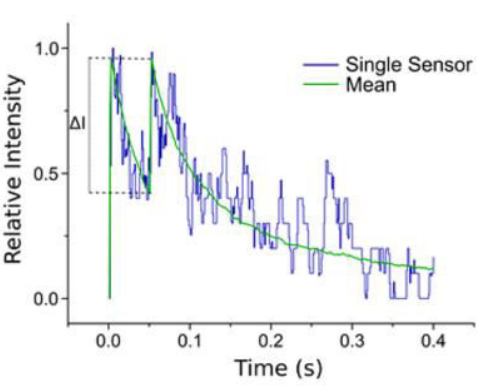

d

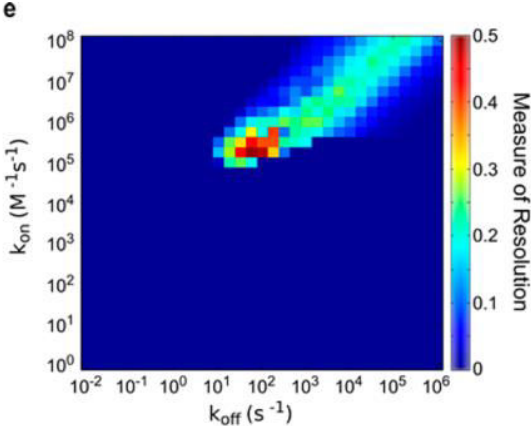

f
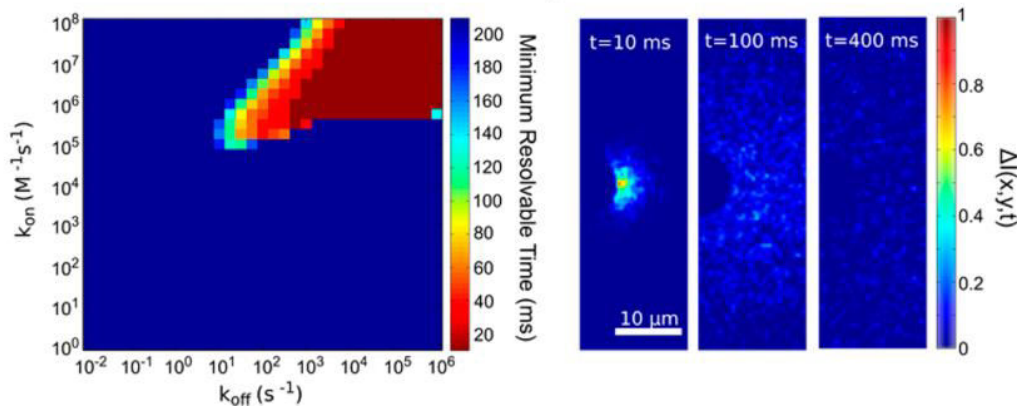

Figure 5. Spatial and temporal resolution as a function of rate constants. (a) Spatial resolution definition. A fixed pixel A ( $d=500 \mathrm{~nm}$ away from the release point) was chosen, and its intensity was compared to the intensity of a second pixel B (distance $d=500 \mathrm{~nm}$ horizontal to pixel A) at $t=10 \mathrm{~ms}$ after analyte release. (b) Phase diagram of the intensity difference between pixel A and B for different rate constants $k_{\text {on }}$ and $k_{\text {off }}(N=10$ simulation runs $)$. The phase diagram shows the spatial resolution and highlights that in a first zone with $k_{\text {on }}=\left[10^{4} ; 10^{6}\right] \mathrm{M}^{-1}$ $\mathrm{s}^{-1}$ and $k_{\text {off }}=\left[10^{-2} ; 10^{2}\right] \mathrm{s}^{-1}$ the pixels can be distinguished. For larger values of $k_{\text {on }}$ and $k_{\text {off }}$, corresponding to a $K_{\mathrm{D}} \approx 1 \mathrm{mM}$, a second zone appears that allows resolving the two pixels. (c) Definition of the temporal resolution. The time $\Delta t$ that is needed until a second release event can be resolved by the same sensor $(\Delta I>3 \times$ noise $)$ is a measure for the temporal resolution. (d) Temporal resolution phase diagram. The minimal time required to distinguish two consecutive release events was plotted for different combinations of $k_{\text {on }}$ and $k_{\text {off }}$ Faster rate constants $k_{\text {on }}$ and $k_{\text {off }}$ naturally lead to better time resolution. (e) Combined spatial (b) and temporal (e) phase diagram. (f) Example for the intensity profile $\Delta I(x, y, t)$ of the best $k_{\text {on }}, k_{\text {off }}$ values found in $(\mathrm{e})\left(k_{\mathrm{on}}=10^{6} \mathrm{M}^{-1} \mathrm{~s}^{-1}, k_{\text {off }}=10^{2} \mathrm{~s}^{-1}\right.$, $\left.\mathrm{t}_{\text {int }}=10 \mathrm{~ms}\right)$. The release point is resolved, and sensors recover quickly.

$3.4 \times 10^{-2} \mathrm{~mol} / \mathrm{L}\left(N_{\mathrm{c}} \approx 85000\right)^{6}$ or $c_{0}=0.735 \mathrm{~mol} / \mathrm{L}\left(N_{\mathrm{c}} \approx 2\right.$ $\left.\left.\times 10^{6}\right)^{7}\right)$. Dopamine is an important neurotransmitter and involved in learning and reward control. ${ }^{42}$ The diffusion constants of small molecules such as dopamine are very similar, and therefore, the expected results for different molecules should be very similar. In general, the number of neurotransmitters per vesicle, the size of vesicles, and the number of released molecules are not well-known. To our knowledge, these numbers represent the best estimate for dopaminergic cells (PC12 and chromaffine cells). Release was accomplished by removing the outer membrane of the vesicle at a given time point $t_{0}$ which allows free diffusion at room temperature $(T=$ $\left.24{ }^{\circ} \mathrm{C}\right)$. The resulting concentration profiles had a time resolution of $\Delta t=1 \mathrm{~ms}$ and a spatial resolution of $100 \mathrm{~nm}$ per pixel.

We assumed that diffusion of the analyte (dopamine) is not affected by the presence of the sensors. Given that the number of released molecules $N \approx 2 \times 10^{6}$ is large compared to the number of sensors per field (around 10000), even fully occupied sensors will not significantly change the analyte concentration. Additionally, in 3D, the concentrations would be even lower.

In Figure $3 \mathrm{~b}-\mathrm{e}$ the impact of the rate constants on the resulting image $\Delta I(x, y, t)$ is shown (see also movies M1-M5). Fast binding $\left(k_{\text {on }}=10^{7} \mathrm{M}^{-1} \mathrm{~s}^{-1}\right)$ and slow unbinding (low $k_{\text {off }}=$
$1 \mathrm{~s}^{-1}$ ) result in a response image that quickly saturates (Figure $3 e)$.

In contrast, if the binding rate constant becomes slower $\left(k_{\text {on }}\right.$ $=10^{3} \mathrm{M}^{-1} \mathrm{~s}^{-1}$ ) only stochastic events reminiscent of blinking can be observed. Rate constants between $k_{\text {on }}=10^{6} \mathrm{M}^{-1} \mathrm{~s}^{-1}$ and $k_{\text {off }}=10^{2} \mathrm{~s}^{-1}$, on the other side, represent the real concentration profile much better (Figure $3 \mathrm{~d}$ ). This observation is also found for single sensors if they are exposed to a rectangular concentration profile (Figure S3). Fast sensors reproduce the rectangular profile, while slower sensors have a response delay.

Another factor that convolutes the response image $\Delta I(x, y, t)$ are the frame rates of the imaging system (Figure 4). A frame rate of $1 \mathrm{~ms}$ results in images that represent the concentration profile shown in Figure $3 \mathrm{a}$ for appropriate rate constants $\left(k_{\mathrm{on}}=\right.$ $\left.10^{6} \mathrm{M}^{-1} \mathrm{~s}^{-1}, k_{\text {off }}=10^{2} \mathrm{~s}^{-1}\right)$. If the frame rate is too slow, information can be lost. For example, at frame rates of $10 \mathrm{~ms}$ (Figure 4b) or $100 \mathrm{~ms}$ (Figure 4c) diffusion leads to a broadening of the release profile. In conclusion, the higher the frame rate the more spatiotemporal details can be resolved in chemical imaging. However, if the sensor signal is not high enough, slower frame rates might be necessary to balance signal/noise and spatiotemporal resolution.

We also tested if arrangement, density, size, and shape of sensors play a role. For that purpose, we simulated different shapes of nanosensors (particles, rods/tubes, and triangles). As can be expected from resolution limit considerations, the shape 
of nanoparticles does not play a role for the response image (Figures S4 and S5). Therefore, the choice of our model sensors (carbon nanotubes) does not affect the results, and the conclusions that are presented here are generic for different nanoparticle shapes.

In addition, we also changed the distance between sensors. As long as the intersensor distance is smaller than the diffraction limit (e.g., $\lambda / 2=980 / 2 \mathrm{~nm}=490 \mathrm{~nm}$ for carbon nanotube emission) there is no difference in response image. This will hold only true as long as the sensors do not change the concentration profile $c_{\mathrm{A}}(x, y, t)$ significantly. Otherwise, the density of the sensors should have an impact on diffusion and the resulting response image.

When individual sensors can be resolved above the resolution limit the image will contain discrete spots of sensors (Figure S4e). This might have advantages because then single-sensor data can be collected. However, spatial resolution is lost, and the signal/noise ratios might suffer. Therefore, we focused on dense arrays (distance smaller than the resolution limit), which is also more in line with the idea of chemical imaging.

Another factor could be the order of the nanosensor pattern. There are methods available to place and functionalize nanoparticles of tunable size and shape in defined distances on different substrates. ${ }^{4-47}$ Therefore, this question is not limited to simulations. However, ordered patterns (distance $d=$ $500 \mathrm{~nm}$ ) show very similar images compared to random patterns with the same number of sensors (Figure S4).

Spatiotemporal Resolution. The simulation procedure allows us to calculate a response image $\Delta I(x, y, t)$ for a given concentration profile $c_{\mathrm{A}}(x, y, t)$. As already shown above, the response image depends on different parameters, but rate constants have the strongest impact.

An important general question is if chemical imaging with nanosensors is able to resolve a given concentration profile. Therefore, we determined the spatial and temporal resolution for different rate constants and created resolution phase diagrams.

One way to define a spatial resolution is to ask the question if two pixels separated by a certain distance show a different response at a given time point (Figure 5a). Here, we chose two pixels in a distance of $500 \mathrm{~nm}$, which corresponds roughly to the Abbe limit of nIR light $(980 \mathrm{~nm})$ for our model sensors (nIR emitting carbon nanotubes). The responses of these two pixels were compared at $t=10 \mathrm{~ms}$ after the release event with an exposure time set to $t_{\text {int }}=10 \mathrm{~ms}$. This resolution definition is different from the typical resolution definition in fluorescence microscopy because it is rather a multidimensional resolution that depends on the concentration profile $c_{\mathrm{A}}(x, y, t), k_{\text {on }}$, and $k_{\text {off }}$ and the chosen time point. Directly after release $(<1 \mathrm{~ms})$ the analyte (e.g., dopamine) will diffuse to pixel $\mathrm{A}$ and cause a response while pixel B is not reached yet. Later $(10 \mathrm{~ms})$, both pixels will experience a certain concentration, and even later, the concentrations become so low that the concentration of the analyte is negligible and the response is mainly determined by unbinding kinetics $\left(k_{\text {off }}\right)$. Therefore, there is not one spatial or temporal resolution. It is rather a multidimensional resolution space that accounts for all aspects. Our simulation procedure covers all aspects but for every representation such as in Figure 5 we have to pick, e.g., a time point or use a certain concentration profile.

Figure $5 \mathrm{~b}$ shows if two adjacent pixels $(500 \mathrm{~nm}$ distance from each other and release point) can be distinguished in a colorcoded phase diagram for different on and off rates. It shows two regimes of rate constants that can distinguish the two pixels: a first, horizontal, regime (between $k_{\text {on }}=\left[10^{4} ; 10^{6}\right] \mathrm{M}^{-1} \mathrm{~s}^{-1}$ and $\left.k_{\text {off }}=\left[10^{-2} ; 10^{2}\right] \mathrm{s}^{-1}\right)$ and a second one that corresponds to an approximately constant dissociation constant $K_{\mathrm{D}} \approx 1 \mathrm{mM}$ (starting at $k_{\mathrm{on}}=3 \times 10^{5} \mathrm{M}^{-1} \mathrm{~s}^{-1}$ and $k_{\text {off }}=3 \times 10^{2} \mathrm{~s}^{-1}$ ). This phase diagram shows for which rate constants the spatial resolution is good enough to distinguish the concentration difference at these two adjacent pixels.

Next, we wanted to get a measure for the temporal resolution. It is high if one can distinguish two temporarily close events. Therefore, we simulated release from two vesicles separated in time by different time periods $\Delta t$. Figure $5 \mathrm{c}$ shows the response curve for one example.

Here, a pixel $d=700 \mathrm{~nm}$ away from the release point was investigated. We defined that the two peaks are distinguishable if the intensity rise $\Delta I$, due to release of the second vesicle, is three times bigger than the average noise level of the data. The phase diagram in Figure 5d shows the minimum difference in time that allows distinguishing of two peaks (exocytosis release events).

Fast rate constants are crucial for the temporal resolution, and there is a regime between $k_{\text {on }}=\left[10^{5} ; 10^{6}\right] \mathrm{M}^{-1} \mathrm{~s}^{-1}$ and $k_{\text {off }}$ $=\left[10^{1} ; 10^{2}\right] \mathrm{s}^{-1}$ that provides temporal resolutions of around $\Delta t<100 \mathrm{~ms}$.

We combined the spatial $(\mathrm{S})$ and temporal $(\mathrm{T})$ resolution diagrams by normalizing them to their minima/maxima to get values between 0 and 1 . Spatial resolution is better for larger values (intensity changes) and temporal resolution for lower values (time periods between distinguishable events). Therefore, we used the "inverted" time resolution and calculated $S \times$ $(1-T)$, which is a measure for the combined spatiotemporal resolution (Figure 5e). This phase diagram shows the rate constants that should enable both a good temporal and spatial resolution. The difference between the combined spatiotemporal phase diagram and the sole spatial heatmap is mainly that too slow off rates are excluded because sensors would remain saturated for too long to respond to a second release event.

Resolution definitions can be formulated in different ways. Our simulation provides more information, and the necessary resolution for a given concentration profile depends mainly on the biological question. In Figure $5 \mathrm{f}$ an example for the best rate constants found in these diagrams is shown for three different time points $\left(k_{\text {on }}=10^{6} \mathrm{M}^{-1} \mathrm{~s}^{-1}, k_{\text {off }}=10^{2} \mathrm{~s}^{-1}, t_{\text {int }}=10 \mathrm{~ms}\right)$. Such a sensor could resolve the concentration profile and quickly decays similar to the fast decay of concentration after exocytosis of signaling molecules.

The scenario in Figure 5a is one way to derive a definition for a resolution. Another way is to ask directly a biological question. For example, for most cells such as neurons it is known what kind of molecules they release (e.g., the neurotransmitter dopamine). In contrast, so far, it is more difficult to resolve where cells release these molecules. This question is extremely important because it finally determines the concentrations of signaling molecules that reach a second cell and how these signals are integrated. Such biological questions can only be answered by chemical-imaging methods.

In order to shed light on this second scenario, we simulated the release of two vesicles from a model cell (Figure 6a). The release sites were placed in different locations on the cell border. If the angle between the release sites is too small for a given sensor array they cannot be resolved anymore and are not identified as two independent sites (Figure 6b). Biologically, it is important to distinguish these events, for example, to 

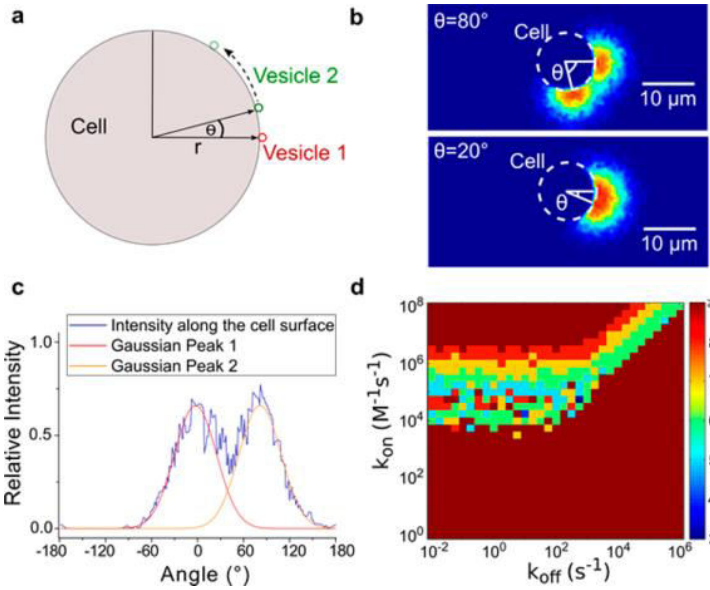

Figure 6. Resolution limit for multiple release events. (a) Resolution definition for multiple release events. Two vesicles at different angles $\theta \in\left[10^{\circ} ; 80^{\circ}\right]$ filled with the analyte (e.g., neurotransmitter) are released by the cell at the same time. (b) Generic intensity profiles for $\theta=80^{\circ}$ (top) and $\theta=20^{\circ}$ (bottom). The release events are resolved $\left(\theta=80^{\circ}\right)$ or not $\left(\theta \neq 80^{\circ}\right)$ depending on the rate constants. (c) Example of an intensity crosssection. To identify the minimum resolvable angle, the response was analyzed at a distance $d=500 \mathrm{~nm}$ from the cell $\left(\mathrm{c}, \theta=80^{\circ}\right)$ The profile was fitted by a double-Gaussian function, and the two peaks were defined as resolvable if $\Delta I(\theta)<\Delta I_{\max } / e$. (d) Phase diagram of angular resolution for different rate constants that resemble the spatial resolution phase diagram in Figure 4. The best resolution was found for $k_{\mathrm{on}}=\left[10^{4} ; 10^{6}\right] \mathrm{M}^{-1} \mathrm{~s}^{-1}$ and $k_{\mathrm{off}}=\left[10^{-2}\right.$; $\left.10^{3}\right] \mathrm{s}^{-1}$ (exposure time $t_{\text {int }}=10 \mathrm{~ms}$ ).

distinguish extra-synaptic release of neurotransmitters and release from cell body or axon/dendrite. The two release events were defined as distinguishable if their intensity profiles

at $d=500 \mathrm{~nm}$ away from the cell show separate peaks. For that purpose, we fitted both profiles (example shown in Figure 6c) with Gaussian functions and determined if the overall intensity at their intersection was $\Delta I<\Delta I_{\max } / e$ or not. With this criterion, it is possible to determine the resolvable angle in a phase diagram (Figure 6d). It is very similar to the phase diagram in Figure $5 \mathrm{~b}$ and shows the same tendencies, which underlines that the spatial resolution definitions we used in Figure 5 are of general relevance.

To go a step further, we simulated the release of multiple vesicles ( $n=20,100000$ dopamine molecules each $)$ in the synaptic cleft between two cells. These numbers were chosen because there are most likely multiple release events and the number of molecules per vesicle is best known from dopaminergic cells and on the same order of magnitude. ${ }^{55}$ For that purpose, we used the electron microscopy images of a dopaminergic neuron to simulate release from a realistic synaptic structure. ${ }^{48}$ The synaptic cleft is very narrow $(d=10$ $\mathrm{nm}$ ), and the geometry is slightly asymmetric.

We chose the best rate constants that we found from the resolution considerations in Figures 5 and $6\left(k_{\text {on }}=10^{6} \mathrm{M}^{-1} \mathrm{~s}^{-1}\right.$ and $\left.k_{\text {off }}=10^{2} \mathrm{~s}^{-1}\right)$. Figure $7 \mathrm{a}$ shows the simulated concentration profile for the first $100 \mathrm{~ms}$. In Figure $7 \mathrm{~b}$, the simulated sensor response images show that release is detected. Figure $7 \mathrm{~b}$ also shows that the subtle asymmetry of the synapse translates into an asymmetric response profile (stronger responses on the left than on the right).

In contrast to Figures 5 and 6, the responses look more pixelated. This can be explained by the size of the synaptic cleft $(300 \mathrm{~nm})$, the sensors $(1 \times 300 \mathrm{~nm})$, and the camera pixels $(200 \mathrm{~nm})$. Therefore, the response is more stochastic, and the images look more pixelated. This is not a technical limitation but reflects the underlying random walk of molecules, which also happens in biological systems. a

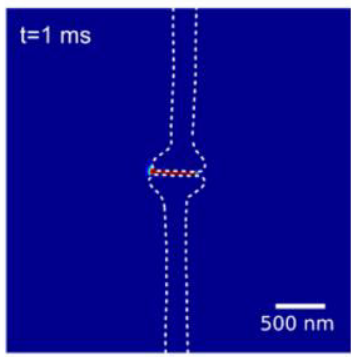

b

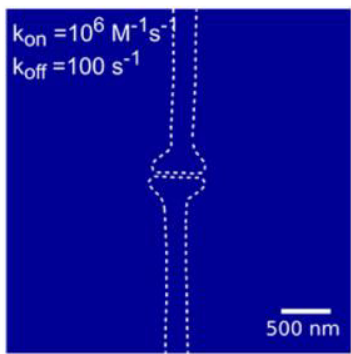

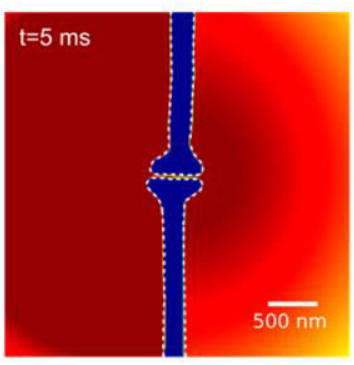

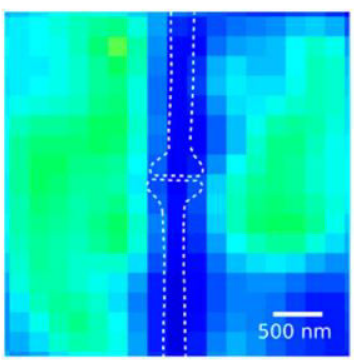

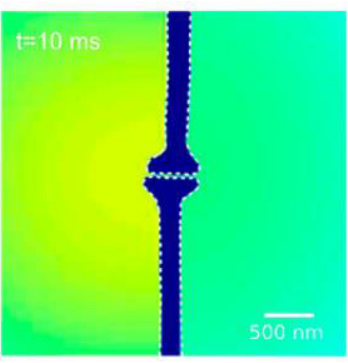

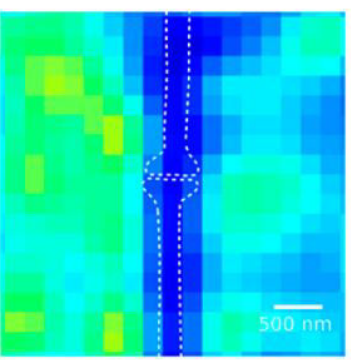

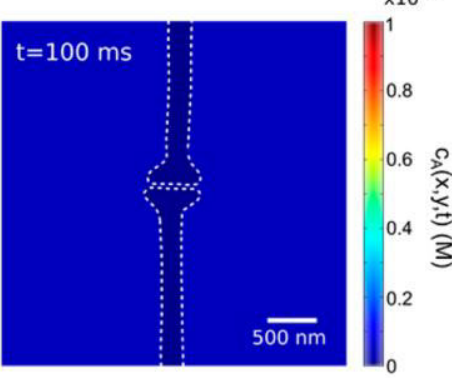

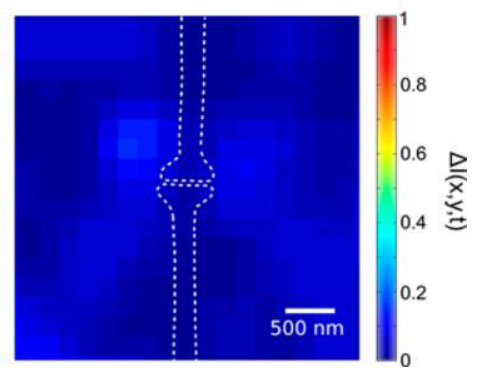

Figure 7. Imaging release of neurotransmitters from a single synapse. (a) Concentration profile of release events inside a synaptic cleft (thickness $d=10 \mathrm{~nm}, 100000$ molecules per release, $n=20$ vesicles) at different time points. (b) Corresponding response images for sensors with rate constants $k_{\mathrm{on}}=10^{6} \mathrm{M}^{-1} \mathrm{~s}^{-1}$ and $k_{\text {off }}=10^{2} \mathrm{~s}^{-1}$. 
An important result of this work is that for imaging of fast processes, such as neurotransmitter release, the kinetics of sensors is crucial. Our results show that fast sensors $\left(k_{\mathrm{on}}=10^{6}\right.$ $\mathrm{M}^{-1} \mathrm{~s}^{-1}$ and $k_{\text {off }}=10^{2} \mathrm{~s}^{-1}$ ) that have especially a fast off-rate and therefore low affinity $\left(K_{\mathrm{D}}=k_{\text {off }} / k_{\mathrm{on}}=100 \mu \mathrm{M}\right)$ provide the best spatiotemporal resolution for the neurotransmitter release events we investigated. However, different concentration profiles will have different optimal kinetics. Therefore, the algorithm presented in this work can be used to calculate the response image $\Delta I(x, y, t)$ for every given experimental or simulated concentration profile $c_{A}(x, y, t)$. The results from this work and the algorithm can be consulted before a new nanosensor is synthesized. Additionally, for sensors of known rate the phase diagrams in Figure 5 are very useful to decide if a biological experiment should be performed at all. Even if a nanosensor does not fall into the region that is able to resolve a given concentration profile rational changes can be implemented without the need of time-consuming trial-and-error experiments.

In principle, the algorithm can be applied to any fluorescent sensor of known position in $2 \mathrm{D}$ or $3 \mathrm{D}$. If nanosensors are, for example, inside cells and not immobilized their movement will affect the response image, but as long as their diffusion during image acquisition is slower than the resolution limit this algorithm is applicable.

Chemical imaging of signaling can be especially important to study communication in cell circuits. Therefore, neurobiological questions such as corelease of different neurotransmitters, extrasynaptic release, neurotransmitter switches, or volume transmission could benefit from such techniques. ${ }^{49-52}$ Another possible application is imaging of chemical communication between immune cells or quorum sensing in bacterial films. ${ }^{53}$

\section{CONCLUSIONS}

We have developed an algorithm to simulate images of fluorescent nanosensor arrays that are exposed to a changing concentration profile. The rate constants of the sensors play a decisive role and resolution limits were calculated and analyzed by preparing phase diagrams. This work can serve as a guideline to engineer nanosensors in order to achieve a desired spatial or temporal resolution. The simulations can also provide insights into what kind of concentration changes can be resolved and what type of biological questions can be asked for a nanosensor array with certain properties (rate constants, shape, size, density).

\section{MATERIALS AND METHODS}

The complete simulation algorithm was implemented in Matlab (R2014a) and will be shared upon request and on our homepage.

Stochastic Simulation of Binding Events on Single Sensors. We used a stochastic simulation algorithm (SSA), similar to the Gillespie algorithm to calculate the number of bound analytes on a nanosensor for a given concentration of analyte at a specific time point. $^{37,39}$ The reaction of a sensor $S$ with an analyte A is bimolecular and described by the rate constants $k_{\text {on }}$ and $k_{\text {off: }}$ :

$$
\mathrm{S}+\mathrm{A} \underset{k_{\text {off }}}{\rightleftharpoons} \mathrm{SA}
$$

The sensor pattern was divided into small subvolumes of an approximately constant analyte concentration and one sensor per subvolume. Thus, for an immobilized sensor $S$ the concentration of $\mathrm{A}$ around $S$ is approximately constant for a small period of time. Because there is only one immobilized sensor per subvolume the time $\tau$ needed for the forward or backward reaction is then stochastically described by

$$
\begin{gathered}
\tau_{\text {on }}=\frac{1}{k_{\text {on }} c_{A}} \ln \left(\frac{1}{r_{\text {on }}}\right) \\
\tau_{\text {off }}=\frac{1}{k_{\text {off }}} \ln \left(\frac{1}{r_{\text {off }}}\right)
\end{gathered}
$$

Here, $r$ is a random number between 0 and 1 and $c_{\mathrm{A}}\left(x_{\mathrm{S}}, y_{\mathrm{S}}, t\right)$ is the concentration of $\mathrm{A}$ close to the sensor at location $\left(x_{\mathrm{S}}, y_{\mathrm{S}}\right)$ and time point $t$. A sensor $S$ might have $n$ binding sites that are described by the binding states $N=\left(\mathrm{B}_{1}, \ldots \mathrm{B}_{n}\right)$. We assumed that they are independent (no cooperativity), which allowed us to implement the following execution steps to simulate the binding/unbinding events within a time interval $\Delta t$ (SSA):

1. Initialize the time $t=t_{0}$ and the state of the binding sites $N$ from the last event or previous test. If $t_{0}=0$, all binding sites are set as "unbound".

2. For "bound" states calculate $\tau_{\text {off }}$ with eq 7 . In the case of an "unbound" state, calculate $\tau_{\text {on }}$, eq 6 , vice versa.

3. If $\tau_{\text {off }}$ or $\tau_{\text {on }}<\Delta t$ change $t \rightarrow t+\tau_{\text {on/off }}$ and switch binding states from $N\left(t=t_{0}\right)$ to $N\left(t=t_{0}+\tau_{\text {on/off }}\right)$

4. Record $N(t)$ and return to step 1 until $\sum \tau_{\text {on }}+\sum \tau_{\text {off }} \geq \Delta t$

As a consequence, the algorithm returns a state distribution $N(t)$ of each sensor-binding site for constant values of $k_{\text {on }}, k_{\text {off }}$ and $c_{\mathrm{A}}\left(x_{\mathrm{S}}, y_{\mathrm{S}}, t\right)$.

The normal Gillespie algorithm relies on a spatially constant concentration profile $c_{\mathrm{A}}(x, y, t)$. Therefore, we divided the concentration profile spatially in subvolumes of edge length $100 \mathrm{~nm}$ and temporarily in small frames with interval lengths of $[t, t+\Delta t]$, in which $c_{\mathrm{A}}\left(x_{\mathrm{S}}, y_{\mathrm{S}}, t\right)$ stays approximately constant. Then we applied for each of these frames the simulation procedure. We chose $\Delta t=1 \mathrm{~ms}$ because in $1 \mathrm{~ms}$ a typical neurotransmitter such as dopamine would diffuse only around $500 \mathrm{~nm} .^{54}$

Intensity Profile. The intensity profile $I_{S}\left(x_{S}, y_{S}, t\right)$ of each sensor depends on the occupation of binding sites $N$. We assumed that we have a turn-on sensor such as the published carbon nanotube sensor for the neurotransmitter dopamine and assigned the starting intensity $I_{0}{ }^{22}$ Then we assumed that each binding site contributes equally to the intensity change $\Delta I$ and normalized it such that the response value of a fully occupied sensor becomes $1\left(\Delta I_{S}=I(N(t)) / I(N=(1,1, \ldots 1))\right.$. Consequently, the mean occupation of binding sites $B_{\mathrm{j}}(t)$ during the time step $\Delta \mathrm{t}$ was calculated.

$$
B_{j}(t)=\left\{\begin{array}{l}
1, \text { if binding site } j \text { is occupied } \\
0, \text { else }
\end{array}\right.
$$

The intensity contribution $I_{j}$ of each binding site $\mathrm{j}$ within the time step $[t, t+\Delta t]$ is then given by

$$
I_{\mathrm{j}}=\frac{1}{\Delta t} \int_{t}^{t+\Delta t} B_{\mathrm{j}}\left(t^{\prime}\right) \mathrm{d} t^{\prime}
$$

This integral describes the fraction of time within $[t, t+\Delta t]$ in which the binding site was occupied and is therefore an appropriate measure of its contribution to the general intensity change by a single site. The relative intensity response $\Delta I_{S}(N, t)$ of the sensor $S$ can accordingly be calculated by simply summing up all $n$ binding site contributions:

$$
\Delta I_{S}\left(x_{s}, y_{s}, t\right)=\frac{1}{n} \sum_{j=1}^{n} I_{j}=\frac{1}{n} \sum_{j=1}^{n} \frac{1}{\Delta t} \int_{t}^{t+\Delta t} B_{j}\left(t^{\prime}\right) \mathrm{d} t^{\prime}
$$

A typical number of bindings sites used in the simulation was $n=10$ (see discussion for details). In summary, $\Delta I_{S}\left(x_{S}, y_{S}, t\right)$ corresponds to the fluorescence change of a single sensor exposed to a concentration profile $c_{\mathrm{A}}(x, y, t)$.

Image of an Array of Sensors and Abbe Limit. To calculate the image of multiple sensors, an array of sensors with known sensor positions was exposed to the concentration profile $c_{\mathrm{A}}(x, y, t)$. Then the SSA algorithm for each sensor $S$ in position $\left(x_{S}, y_{S}\right)$ was performed to 
yield $\Delta I_{S}\left(x_{S}, y_{S}, t\right)$. To account for the diffraction limit, every sensor intensity $\Delta I_{\mathrm{S}}\left(x_{\mathrm{S}}, y_{\mathrm{S}}, t\right)$ was overlaid with $(r=1.2 \mu \mathrm{m})$ airy disks using the formula

$$
r_{\text {airy }}(\mu \mathrm{m})=1.22 \times \lambda(\mu \mathrm{m}) \times \mathrm{f} / \#
$$

Here, $\lambda=0.98 \mu \mathrm{m}$ was used as a typical emission wavelength of singlewalled carbon nanotubes and an $f$-number of $f / \#=1$ for typical microscope setups. The overlay of all airy disks was performed by setting the first order of the besselfunction (of the first kind) $J_{0}$

$$
J_{0}(r)=\sum_{k=0}^{\infty} \frac{\left(\frac{r}{4}\right)^{k}}{k ! \Gamma(k+1)}
$$

radially around each sensor pixel and using $r=r_{\text {airy }}$ as the radius of the overlaid disk. Finally, we multiplied it with the sensors original intensity value and divided it by the maximum intensity to regain relative values $\Delta I_{\mathrm{A}}(x, y, t)$ between 0 and 1 .

Further, we assumed a spatial resolution limit of the camera chip of $200 \mathrm{~nm}$ per pixel as an approximate value for near-infrared cameras (http://www.jai.com/en/products/nearinfrared). For that purpose, we superimposed the intensity image with a Cartesian grid, calculated the average intensity within each of the $200 \times 200 \mathrm{~nm}^{2}$ boxes, and recalculated each pixel. The result is an image of sensor responses $\Delta I(x, y, t)$ with $200 \mathrm{~nm}$ pixel size and a time resolution $t_{\text {int }}>1 \mathrm{~ms}$ defined by the camera frame rate.

Simulation of the Concentration Profiles and Neurotransmitter Release. Spatiotemporal concentration profiles of neurotransmitter release events were simulated with COMSOL Multiphysics Modeling Software (v. 4.1). For a minimal exocytosis system, we modeled the cell as a non-deformable, immobile circle with a radius of $r=5 \mu \mathrm{m}$ put on a $50 \times 50 \mu \mathrm{m}^{2}$ surface coated with sensors. Vesicles were modeled as small spheres (radius $=0.1 \mu \mathrm{m}$ ) and were filled with a certain number of molecules, (e.g., $N=1.85 \times 10^{6}$ molecules as estimated for chromaffin cells). ${ }^{55}$ Furthermore, as a model neurotransmitter we used dopamine with the appropriate diffusion constant $\left(D=8.3 \times 10^{-7} \mathrm{~cm}^{2} / \mathrm{s}\right){ }^{50}$ Typically, at time point $t_{0}$, the vesicle membrane was set to be no barrier anymore and the concentration profile $c_{\mathrm{A}}(x, y, t)$ was simulated over a given time period.

\section{ASSOCIATED CONTENT}

\section{S Supporting Information}

The Supporting Information is available free of charge on the ACS Publications website at DOI: 10.1021/acsnano.7b00569.

Additional control experiments and simulations (PDF)

Time-lapse movie of concentration simulation (AVI)

Time-lapse movie of slow binding rates (AVI)

Time-lapse movie of slow unbinding rates (AVI)

Time-lapse movie of well-chosen values (AVI)

Time-lapse movie of too fast binding rates (AVI)

\section{AUTHOR INFORMATION}

\section{Corresponding Author}

*E-mail: skruss@uni-goettingen.de.

\section{ORCID $\odot$}

Sebastian Kruss: 0000-0003-0638-9822

\section{Notes}

The authors declare no competing financial interest.

\section{ACKNOWLEDGMENTS}

We thank the Fonds der chemischen Industrie (FCI) for financial support (Liebig fellowship to S.K.). This project was supported by the state of Lower Saxony (life@nano). Part of this work was supported by the Cluster of Excellence and DFG Research Center Nanoscale Microscopy and Molecular Physiology of the Brain.

\section{REFERENCES}

(1) Evans, C. L.; Xie, X. S. Coherent Anti-Stokes Raman Scattering Microscopy: Chemical Imaging for Biology and Medicine. Annu. Rev. Anal. Chem. 2008, 1, 883-909.

(2) Bocklitz, T. W.; Guo, S.; Ryabchykov, O.; Vogler, N.; Popp, J. Raman Based Molecular Imaging and Analytics: A Magic Bullet for Biomedical Applications!? Anal. Chem. 2016, 88, 133-151.

(3) Berezin, M. Y.; Achilefu, S. Fluorescence Lifetime Measurements and Biological Imaging. Chem. Rev. 2010, 110, 2641-2684.

(4) da Cunha, M. M.; Trepout, S.; Messaoudi, C.; Wu, T. D.; Ortega, R.; Guerquin-Kern, J. L.; Marco, S. Overview of Chemical Imaging Methods to Address Biological Questions. Micron 2016, 84, 23-36.

(5) Alberts, B. Molecular Biology of the Cell; Taylor \& Francis Ltd, 2014

(6) Schaferling, M. The Art of Fluorescence Imaging with Chemical Sensors. Angew. Chem., Int. Ed. 2012, 51, 3532-3554.

(7) Wolfbeis, O. S. An Overview of Nanoparticles Commonly Used in Fluorescent Bioimaging. Chem. Soc. Rev. 2015, 44, 4743-4768.

(8) Ha, T. Probing Nature's Nanomachines One Molecule at a Time. Biophys. J. 2016, 110, 1004-1007.

(9) Klein, C.; Engler, R. H.; Henne, U.; Sachs, W. E. Application of Pressure-Sensitive Paint for Determination of the Pressure Field and Calculation of the Forces and Moments of Models in a Wind Tunnel. Exp. Fluids 2005, 39, 475-483.

(10) Wang, X. D.; Meier, R. J.; Link, M.; Wolfbeis, O. S. Photographing Oxygen Distribution. Angew. Chem., Int. Ed. 2010, 49, 4907-4909.

(11) Schreml, S.; Meier, R. J.; Wolfbeis, O. S.; Landthaler, M.; Szeimies, R. M.; Babilas, P. 2d Luminescence Imaging of $\mathrm{Ph}$ in Vivo. Proc. Natl. Acad. Sci. U. S. A. 2011, 108, 2432-2437.

(12) Stosiek, C.; Garaschuk, O.; Holthoff, K.; Konnerth, A. In Vivo Two-Photon Calcium Imaging of Neuronal Networks. Proc. Natl. Acad. Sci. U. S. A. 2003, 100, 7319-7324.

(13) Alivisatos, A. P.; Andrews, A. M.; Boyden, E. S.; Chun, M.; Church, G. M.; Deisseroth, K.; Donoghue, J. P.; Fraser, S. E.; Lippincott-Schwartz, J.; Looger, L. L.; Masmanidis, S.; McEuen, P. L.; Nurmikko, A. V.; Park, H.; Peterka, D. S.; Reid, C.; Roukes, M. L.; Scherer, A.; Schnitzer, M.; Sejnowski, T. J.; Shepard, K. L.; Tsao, D.; Turrigiano, G.; Weiss, P. S.; Xu, C.; Yuste, R.; Zhuang, X. Nanotools for Neuroscience and Brain Activity Mapping. ACS Nano 2013, 7, $1850-1866$.

(14) Andrews, A. M. The Future of Monitoring Molecules. ACS Chem. Neurosci. 2015, 6, 1-2.

(15) Wightman, R. M. Monitoring Molecules: Insights and Progress. ACS Chem. Neurosci. 2015, 6, 5-7.

(16) Andrews, A. M. The Brain Initiative: Toward a Chemical Connectome. ACS Chem. Neurosci. 2013, 4, 645-645.

(17) Liang, R.; Broussard, G. J.; Tian, L. Imaging Chemical Neurotransmission with Genetically Encoded Fluorescent Sensors. ACS Chem. Neurosci. 2015, 6, 84-93.

(18) Borisov, S. M.; Wolfbeis, O. S. Optical Biosensors. Chem. Rev. 2008, 108, 423-461.

(19) Polo, E.; Kruss, S. Nanosensors for Neurotransmitters. Anal. Bioanal. Chem. 2016, 408, 2727-2741.

(20) Mu, B.; Zhang, J.; McNicholas, T. P.; Reuel, N. F.; Kruss, S.; Strano, M. S. Recent Advances in Molecular Recognition Based on Nanoengineered Platforms. Acc. Chem. Res. 2014, 47, 979-988.

(21) Kruss, S.; Hilmer, A. J.; Zhang, J.; Reuel, N. F.; Mu, B.; Strano, M. S. Carbon Nanotubes as Optical Biomedical Sensors. Adv. Drug Delivery Rev. 2013, 65, 1933-1950.

(22) Kruss, S.; Landry, M. P.; Vander Ende, E.; Lima, B. M.; Reuel, N. F.; Zhang, J.; Nelson, J.; Mu, B.; Hilmer, A.; Strano, M. Neurotransmitter Detection Using Corona Phase Molecular Recognition on Fluorescent Single-Walled Carbon Nanotube Sensors. J. Am. Chem. Soc. 2014, 136, 713-724.

(23) Zhang, J.; Kruss, S.; Hilmer, A. J.; Shimizu, S.; Schmois, Z.; De La Cruz, F.; Barone, P. W.; Reuel, N. F.; Heller, D. A.; Strano, M. S. A Rapid, Direct, Quantitative, and Label-Free Detector of Cardiac 
Biomarker Troponin T Using near-Infrared Fluorescent Single-Walled Carbon Nanotube Sensors. Adv. Healthcare Mater. 2014, 3, 412-423. (24) Polo, E.; Kruss, S. Impact of Redox-Active Molecules on the Fluorescence of Polymer-Wrapped Carbon Nanotubes. J. Phys. Chem. C 2016, 120, 3061-3070.

(25) Reuel, N. F.; Grassbaugh, B.; Kruss, S.; Mundy, J. Z.; Opel, C.; Ogunniyi, A. O.; Egodage, K.; Wahl, R.; Helk, B.; Zhang, J.; Kalcioglu, Z. I.; Tvrdy, K.; Bellisario, D. O.; Mu, B.; Blake, S. S.; Van Vliet, K. J.; Love, J. C.; Wittrup, K. D.; Strano, M. S. Emergent Properties of Nanosensor Arrays: Applications for Monitoring Igg Affinity Distributions, Weakly Affined Hypermannosylation, and Colony Selection for Biomanufacturing. ACS Nano 2013, 7, 7472-7482.

(26) Bisker, G.; Dong, J.; Park, H. D.; Iverson, N. M.; Ahn, J.; Nelson, J. T.; Landry, M. P.; Kruss, S.; Strano, M. S. Protein-Targeted Corona Phase Molecular Recognition. Nat. Commun. 2016, 7, 10241.

(27) Nelson, J. T.; Kim, S.; Reuel, N. F.; Salem, D. P.; Bisker, G.; Landry, M. P.; Kruss, S.; Barone, P. W.; Kwak, S.; Strano, M. S. Mechanism of Immobilized Protein a Binding to Immunoglobulin G on Nanosensor Array Surfaces. Anal. Chem. 2015, 87, 8186-8193.

(28) Wong, M. H.; Giraldo, J. P.; Kwak, S. Y.; Koman, V. B.; Sinclair, R.; Lew, T. T.; Bisker, G.; Liu, P.; Strano, M. S. Nitroaromatic Detection and Infrared Communication from Wild-Type Plants Using Plant Nanobionics. Nat. Mater. 2016, 16, 264-272.

(29) Kruss, S.; Salem, D. P.; Vukovic, L.; Lima, B.; Vander Ende, E.; Boyden, E. S.; Strano, M. S. High-Resolution Imaging of Cellular Dopamine Efflux Using a Fluorescent Nanosensor Array. Proc. Natl. Acad. Sci. U. S. A. 2017, 114, 1789-1794.

(30) Bisker, G.; Ahn, J.; Kruss, S.; Ulissi, Z. W.; Salem, D. P.; Strano, M. S. A Mathematical Formulation and Solution of the Cophmore Inverse Problem for Helically Wrapping Polymer Corona Phases on Cylindrical Substrates. J. Phys. Chem. C 2015, 119, 13876-13886.

(31) Landry, M. P.; Vukovic, L.; Kruss, S.; Bisker, G.; Landry, A. M.; Islam, S.; Jain, R.; Schulten, K.; Strano, M. S. Comparative Dynamics and Sequence Dependence of DNA and Rna Binding to Single Walled Carbon Nanotubes. J. Phys. Chem. C 2015, 119, 10048-10058.

(32) Roxbury, D.; Jena, P. V.; Shamay, Y.; Horoszko, C. P.; Heller, D. A. Cell Membrane Proteins Modulate the Carbon Nanotube Optical Bandgap Via Surface Charge Accumulation. ACS Nano 2016, 10, 499506.

(33) Wolfbeis, O. S. Sensor Paints. Adv. Mater. 2008, 20, 3759-3763. (34) Sun, L. N.; Yu, J. B.; Peng, H. S.; Zhang, J. Z.; Shi, L. Y.; Wolfbeis, O.S. Temperature-Sensitive Luminescent Nanoparticles and Films Based on a Terbium (Iii) Complex Probe. J. Phys. Chem. C 2010, 114, 12642-12648.

(35) Nareoja, T.; Deguchi, T.; Christ, S.; Peltomaa, R.; Prabhakar, N.; Fazeli, E.; Perala, N.; Rosenholm, J. M.; Arppe, R; Soukka, T.; Schaeferling, M. Ratiometric Sensing and Imaging of Intracellular Ph Using Polyethyleneimine-Coated Photon Upconversion Nanoprobes. Anal. Chem. 2017, 89, 1501-1508.

(36) Jin, H.; Heller, D. A.; Kalbacova, M.; Kim, J. H.; Zhang, J.; Boghossian, A. A.; Maheshri, N.; Strano, M. S. Detection of SingleMolecule H2o2 Signalling from Epidermal Growth Factor Receptor Using Fluorescent Single-Walled Carbon Nanotubes. Nat. Nanotechnol. 2010, 5, 302-309.

(37) Gillespie, D. T. Stochastic Simulation of Chemical Kinetics. Annu. Rev. Phys. Chem. 2007, 58, 35-55.

(38) Squires, T. M.; Messinger, R. J.; Manalis, S. R. Making It Stick: Convection, Reaction and Diffusion in Surface-Based Biosensors. Nat. Biotechnol. 2008, 26, 417-426.

(39) Gillespie, D. T. Exact Stochastic Simulation of Coupled Chemical Reactions. J. Phys. Chem. 1977, 81, 2340-2361.

(40) Boghossian, A. A.; Zhang, J.; Le Floch-Yin, F. T.; Ulissi, Z. W.; Bojo, P.; Han, J. H.; Kim, J. H.; Arkalgud, J. R.; Reuel, N. F.; Braatz, R. D.; Strano, M. S. The Chemical Dynamics of Nanosensors Capable of Single-Molecule Detection. J. Chem. Phys. 2011, 135, 084124.

(41) Cognet, L.; Tsyboulski, D. A.; Rocha, J. D.; Doyle, C. D.; Tour, J. M.; Weisman, R. B. Stepwise Quenching of Exciton Fluorescence in Carbon Nanotubes by Single-Molecule Reactions. Science 2007, 316, 1465-1468.
(42) Schultz, W. Multiple Dopamine Functions at Different Time Courses. Annu. Rev. Neurosci. 2007, 30, 259-288.

(43) Kruss, S.; Wolfram, T.; Martin, R.; Neubauer, S.; Kessler, H. Spatz, J. P. Stimulation of Cell Adhesion at Nanostructured Teflon Interfaces. Adv. Mater. 2010, 22, 5499-5506.

(44) Kruss, S.; Srot, V.; van Aken, P. A.; Spatz, J. P. Au-Ag Hybrid Nanoparticle Patterns of Tunable Size and Density on Glass and Polymeric Supports. Langmuir 2012, 28, 1562-1568.

(45) Kruss, S.; Erpenbeck, L.; Amschler, K.; Mundinger, T. A. Boehm, H.; Helms, H. J.; Friede, T.; Andrews, R. K.; Schon, M. P.; Spatz, J. P. Adhesion Maturation of Neutrophils on Nanoscopically Presented Platelet Glycoprotein Ibalpha. ACS Nano 2013, 7, 99849996.

(46) Kruss, S.; Erpenbeck, L.; Schon, M. P.; Spatz, J. P. Circular, Nanostructured and Biofunctionalized Hydrogel Microchannels for Dynamic Cell Adhesion Studies. Lab Chip 2012, 12, 3285-3289.

(47) Amschler, K.; Erpenbeck, L.; Kruss, S.; Schon, M. P. Nanoscale Integrin Ligand Patterns Determine Melanoma Cell Behavior. ACS Nano 2014, 8, 9113-9125.

(48) Karayannis, T.; Au, E.; Patel, J. C.; Kruglikov, I.; Markx, S.; Delorme, R.; Heron, D.; Salomon, D.; Glessner, J.; Restituito, S.; Gordon, A.; Rodriguez-Murillo, L.; Roy, N. C.; Gogos, J. A.; Rudy, B.; Rice, M. E.; Karayiorgou, M.; Hakonarson, H.; Keren, B.; Huguet, G.; Bourgeron, T.; Hoeffer, C.; Tsien, R. W.; Peles, E.; Fishell, G. Cntnap4 Differentially Contributes to Gabaergic and Dopaminergic Synaptic Transmission. Nature 2014, 511, 236-240.

(49) Okubo, Y.; Sekiya, H.; Namiki, S.; Sakamoto, H.; Inuma, S.; Yamasaki, M.; Watanabe, M.; Hirose, K.; Iino, M. Imaging Extrasynaptic Glutamate Dynamics in the Brain. Proc. Natl. Acad. Sci. U. S. A. 2010, 107, 6526-6531.

(50) Cragg, S. J.; Rice, M. E. Dancing Past the Dat at a Da Synapse. Trends Neurosci. 2004, 27, 270-277.

(51) Hnasko, T. S.; Edwards, R. H. Neurotransmitter Corelease Mechanism and Physiological Role. Annu. Rev. Physiol. 2012, 74, 225243.

(52) Dulcis, D.; Jamshidi, P.; Leutgeb, S.; Spitzer, N. C. Neurotransmitter Switching in the Adult Brain Regulates Behavior. Science 2013, 340, 449-453.

(53) Camilli, A.; Bassler, B. L. Bacterial Small-Molecule Signaling Pathways. Science 2006, 311, 1113-1116.

(54) Trouillon, R.; Lin, Y.; Mellander, L. J.; Keighron, J. D.; Ewing, A G. Evaluating the Diffusion Coefficient of Dopamine at the Cell Surface During Amperometric Detection: Disk Vs. Ring Microelectrodes. Anal. Chem. 2013, 85, 6421-6429.

(55) Dunevall, J.; Fathali, H.; Najafinobar, N.; Lovric, J.; Wigstrom, J.; Cans, A. S.; Ewing, A. G. Characterizing the Catecholamine Content of Single Mammalian Vesicles by Collision-Adsorption Events at an Electrode. J. Am. Chem. Soc. 2015, 137, 4344-4346. 


\section{Kinetic Requirements for Spatiotemporal Chemical Imaging with Nanosensors}

Daniel Meyer ${ }^{1,2}$, Annika Hagemann ${ }^{1,2}$, Sebastian Kruss ${ }^{1,2 *}$

${ }^{1}$ Institute of Physical Chemistry, Göttingen University, Germany

${ }^{2}$ Center for Nanoscale Microscopy and Molecular Physiology of the Brain (CNMPB),

Göttingen, Germany

*Corresponding author: Sebastian Kruss, email: skruss@uni-goettingen.de
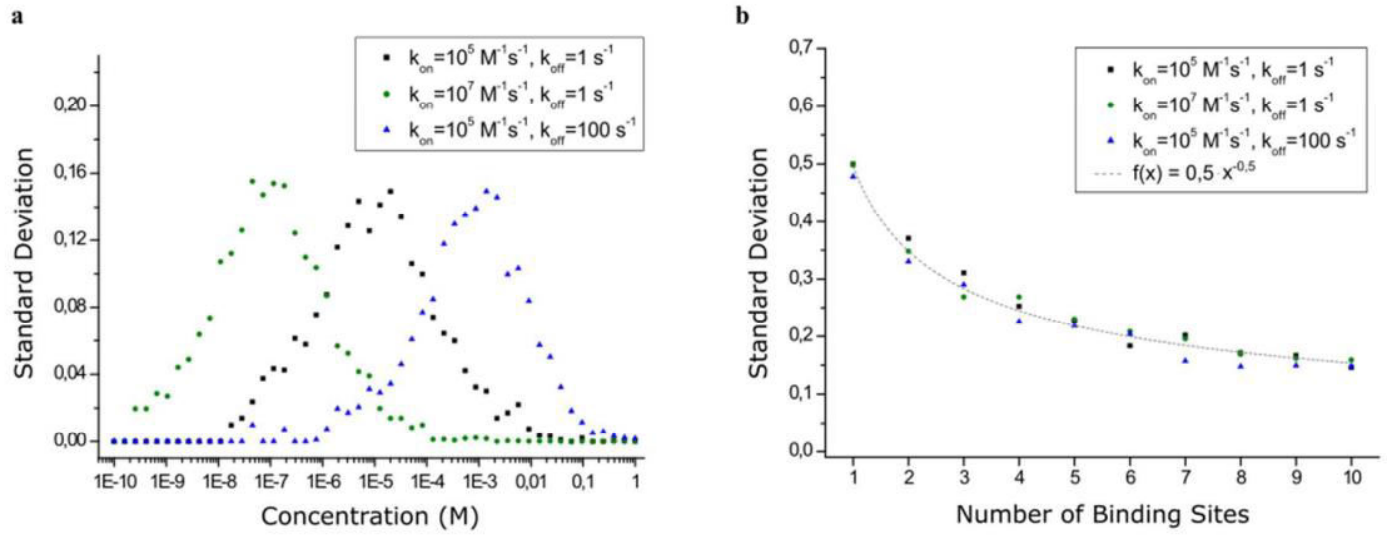

Figure S1: Stochastic fluctuations in fluorescence response. a, Relative intensity fluctuations (defined as standard deviation for $n=100$ simulation runs) as a function of the analyte concentration and the rate constants $k_{\text {on }}$ and $k_{o f f} \boldsymbol{b}$, Relative fluctuations for different numbers of total binding sites. As the intensity of the sensor is defined by the average number of bound binding sites, the intensity fluctuation decrease by increasing the number of binding sites. 
a
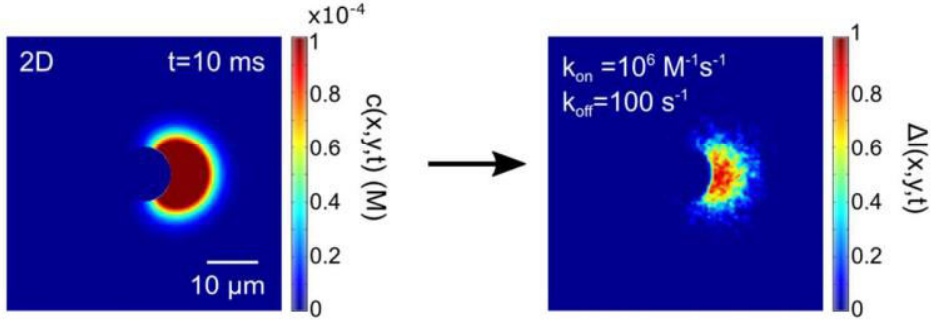

b

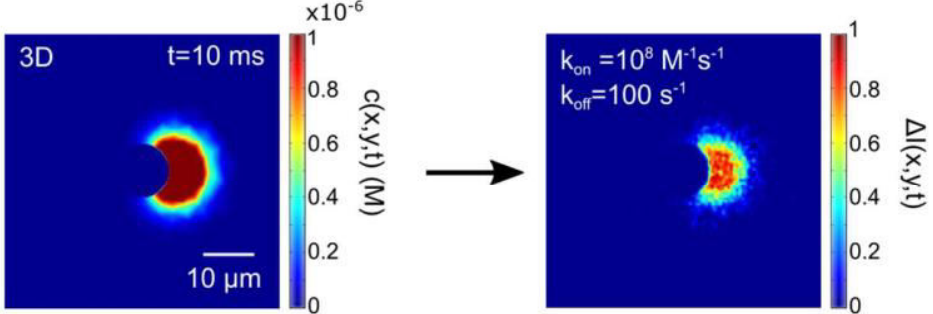

Figure S2: Comparison of $2 D$ versus $3 D$ simulation. a, Concentration profile of a $2 D$ diffusion simulation and the corresponding intensity profile for given values of $k_{\text {on }}$ and $k_{\text {off. }} \boldsymbol{b}$, The concentration profiles in $3 D$ are qualitatively similar although the absolute concentrations are different. The response images are similar if the rate constants are changed accordingly. 

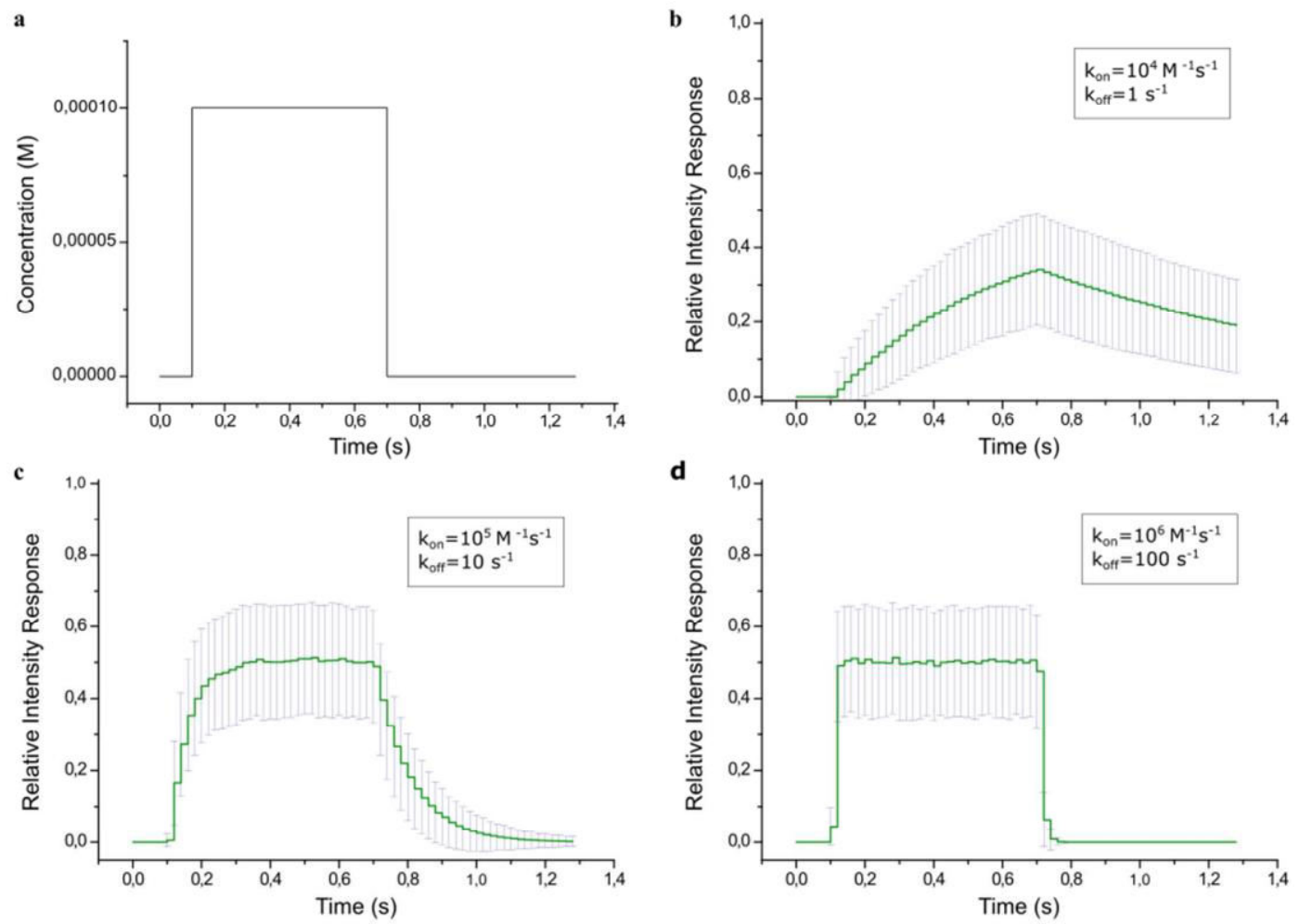

Figure S3: Mean single sensor response to a rectangular concentration profile. a, Concentration profile. $\boldsymbol{b}$-d, Average response of a single binding site for different values of $k_{\text {on }}$ and $k_{\text {off }}$ but equivalent $K_{D}=10^{-4} \mathrm{M}$. For bigger values of $k_{\text {on }}$ and $k_{\text {off, }}$, the response time of the sensor becomes smaller. Bigger values of $k_{\text {on }}$ lead to a faster binding of an analyte until a maximum probability $p_{\text {on }} \approx 1$ is reached. If also $k_{\text {off }}$ reaches a significant level the disassociation probability becomes high $\left(p_{\text {off }} \approx 1\right)$ leading to an average intensity response of 0.5 ( $T_{\text {on }}$ and $\boldsymbol{T}_{\text {off }}$ have equivalent lengths). Bigger $k_{\text {off }}$ values, however, also lead to fast unbinding events. Error bars are standard deviations for $n=100$ simulation runs. 


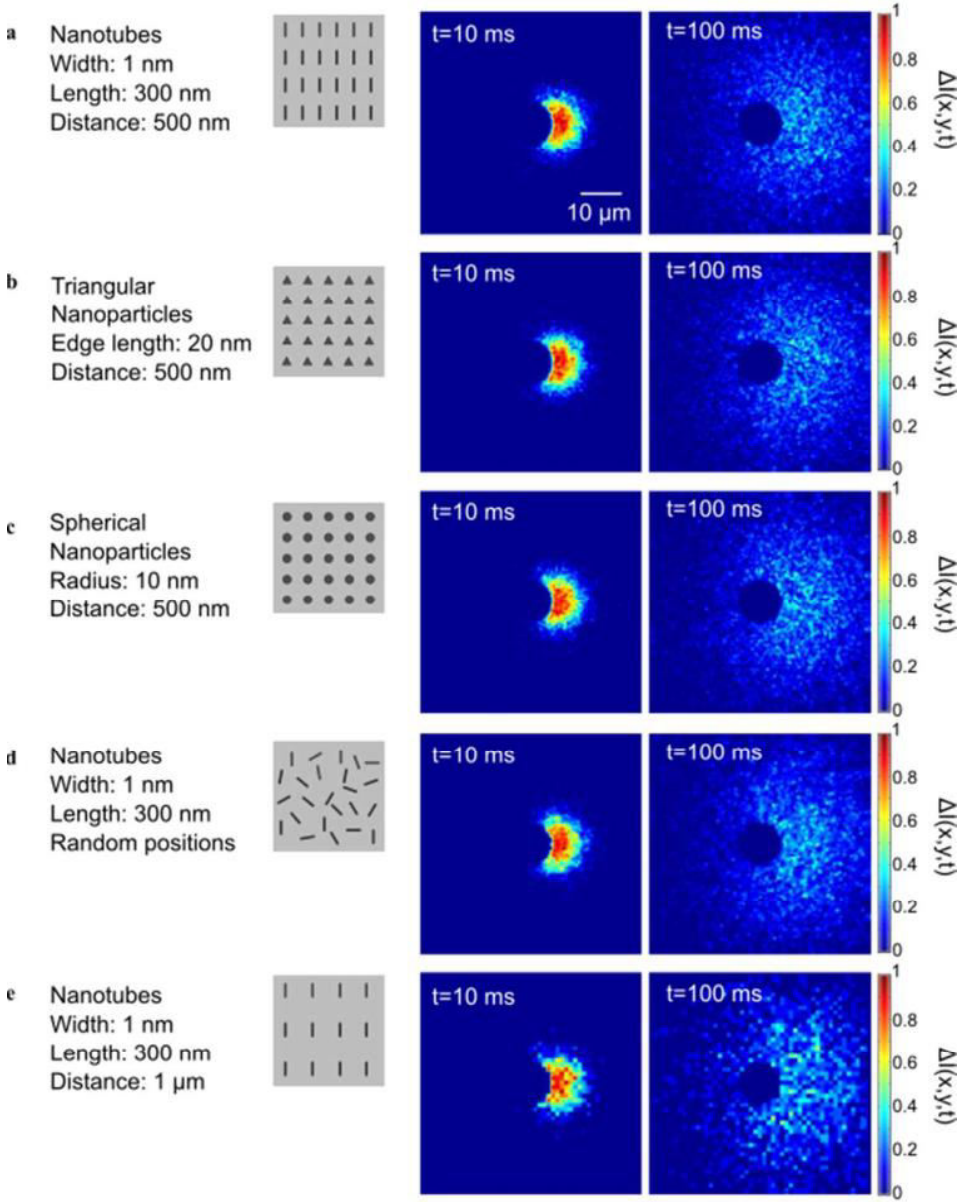

Figure S4: Impact of shape and array geometry on response images. Different nanoparticle shapes and distances between the nanosensors were used. However, no or only subtle differences could be found in the resulting intensity profiles $\Delta \mathbf{I}(x, y, t)\left(t_{\text {int }}=10\right.$ $m s$ ). a-c, Impact of sensor shape. Due to the resolution limit (airy disk) rod like, triangular and spherical nanoparticles show the same response. $d$, The order of the sensors does not have an impact if the density of sensor is high (here $n=9801$ ). e, If distances between sensors get larger the response image becomes more stochastic $\left(k_{o n}=10^{6} \mathrm{M}^{-1} \mathrm{~s}^{-1}, k_{\text {off }}=10^{2} \mathrm{~s}^{-1}\right)$. 


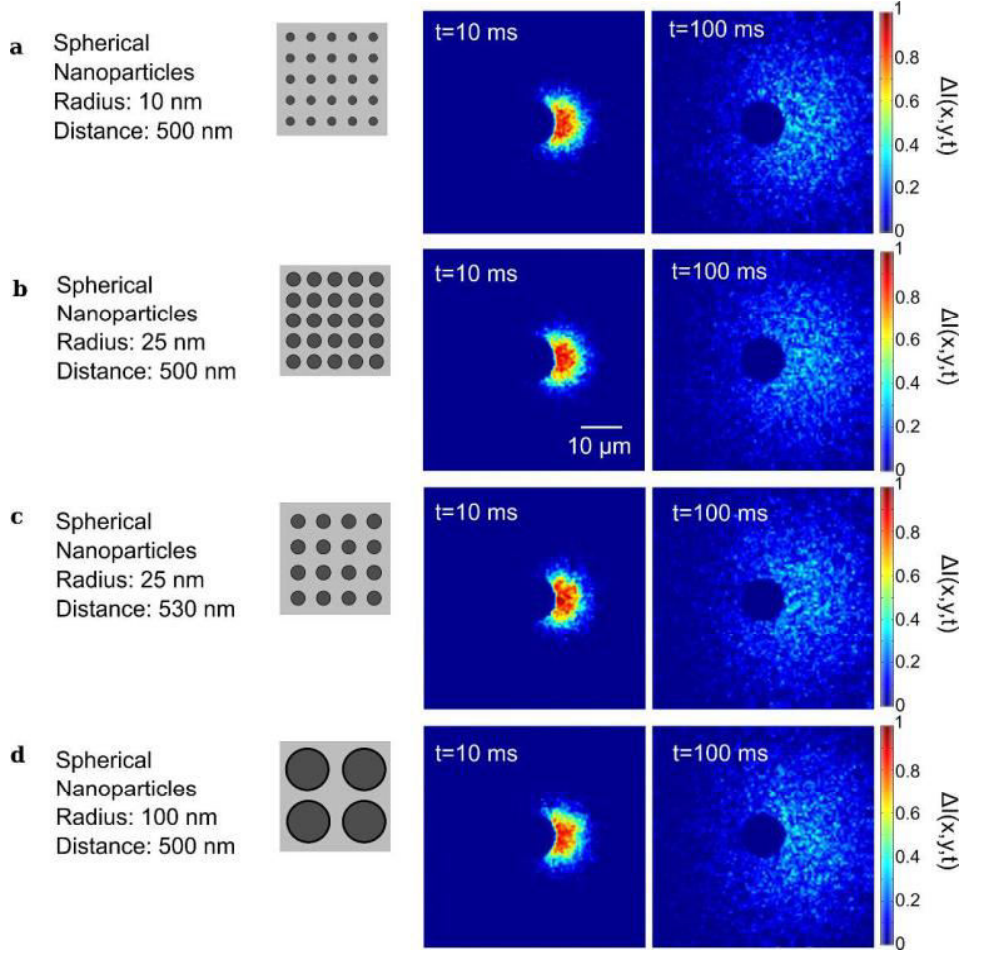

Figure S5: Impact of sensor size on response images. The size of spherical nanosensors was changed to assess its impact on fluorescence response images at different time points $(t=10 \mathrm{~ms}, t=100 \mathrm{~ms})$. Similar to figure $S 4$ no relevant differences were found. a, Radius $r=10 \mathrm{~nm}$ and distance $d=500 \mathrm{~nm}$ to its next neighbor. $\boldsymbol{b}, r=25 \mathrm{~nm}$ and $d=$ $500 \mathrm{~nm} c, r=25 \mathrm{~nm}$ and $d=530 \mathrm{~nm}$ to have the same distance between the edge of two sensors. $\boldsymbol{d}, r=100 \mathrm{~nm}, d=500 \mathrm{~nm}$. 


\section{Supplementary movies}

Movie M1: Time-lapse movie of the corresponding concentration profile used in figures 3-6. As described in the materials \& methods part, a COMSOL simulation software was used to simulate diffusion. A $r=5 \mu \mathrm{m}$ circle was put into the center of a $50 \times 50 \mu^{2}$ sensor area to mimic a cell body. Analytes (typically $N=1.85 \times 10^{6}$ ) were then released from a hypothetical vesicle.

Movie M2: Time-lapse movie of the response image of the sensor array $\left(k_{o n}=10^{3} \mathrm{M}^{-1} \mathrm{~s}^{-1}\right.$, $k_{\text {off }}=1 \mathrm{~s}^{-1}$ ).

Movie M3: Time-lapse movie of the response image of the sensor array $\left(k_{o n}=10^{5} \mathrm{M}^{-1} \mathrm{~s}^{-1}\right.$, $k_{\text {off }}=1 \mathrm{~s}^{-1}$ ).

Movie M4: Time-lapse movie of the response image of the sensor array $\left(k_{o n}=10^{6} M^{-1} \mathrm{~s}^{-1}\right.$ and $k_{\text {off }}=10^{2} \mathrm{~s}^{-1}$ ).

Movie M 5: Time-lapse movie of the response image of the sensor array $\left(k_{o n}=10^{7} \mathrm{M}^{-1} \mathrm{~s}^{-1}\right.$, $k_{\text {off }}=1 s^{-1}$ ). 


\subsection{Manuscript 3}

\section{Programmed Transport and Release of Nanoscale Cargo by Immune Cells}

Prepared for publication

Daniel Meyer ${ }^{1}$, Saba Telele ${ }^{1}$, Anna Zelena ${ }^{3}$, Elsa Neubert ${ }^{2}$, Robert Nissler ${ }^{1}$, Florian Mann $^{1}$, Julia Grandke ${ }^{2}$, Luise Erpenbeck ${ }^{2}$, Sarah Köster ${ }^{3} \&$ Sebastian Kruss ${ }^{1}$

${ }^{1}$ Institute of Physical Chemistry, Göttingen University, Göttingen 37077, Germany

${ }^{2}$ Department of Dermatology, Venerology and Allergology, University Medical Center, Göttingen University, Göttingen 37075, Germany

${ }^{3}$ Institute of X-Ray Physics, Göttingen University, Göttingen 37077, Germany 


\title{
Programmed transport and release of nanoscale cargo by immune cells
}

Daniel Meyer ${ }^{1}$, Saba Telele ${ }^{1}$, Anna Zelená ${ }^{3}$, Elsa Neubert ${ }^{2}$, Robert Nissler ${ }^{1}$, Florian Mann $^{1}$, Julia Grandke ${ }^{2}$, Luise Erpenbeck ${ }^{2}$, Sarah Köster ${ }^{3}$, Sebastian Kruss ${ }^{1 *}$

${ }^{1}$ Institute of Physical Chemistry, Göttingen University, Germany

${ }^{2}$ Department of Dermatology, University Medical Center, Göttingen University, Germany

${ }^{3}$ Institute of X-Ray Physics, Göttingen University, Germany

${ }^{*}$ Correspondence should be addressed to S.K. (email: skruss@uni-goettingen.de)

\begin{abstract}
Transport and delivery of nanoscale materials are crucial for many applications in biomedicine. Here, recent studies elucidated the great potential cell-mediated cargo transport can have, even though controlled uptake, transport and release of functionalized objects remain challenging. In this study, we use human immune cells (neutrophilic granulocytes, neutrophils) and program them to perform these tasks. For this purpose, we let neutrophils phagocytose carbon nanotube-based fluorescent sensors and show that these cells still migrate, follow chemical gradients and respond to inflammatory signals. To program release, we additionally make use of neutrophil extracellular trap formation (NETosis), a novel cell death mechanism that leads to chromatin swelling and subsequent rupture of the cellular membrane. In doing so, the time point of cargo release through NETosis is controlled by the initial concentration of stimulants, such as phorbol 12-myristate-13acetate (PMA) and lipopolysaccharide (LPS). We show that at intermediate concentrations of LPS $(100 \mu \mathrm{g} / \mathrm{ml})$ cells continue to migrate and follow gradients and surface cues for around 30 minutes until they stop and release their cargo. In this process, the transported and released carbon nanotube sensors are still functional as shown by subsequent detection of the neurotransmitter dopamine and the reactive oxygen species $\mathrm{H}_{2} \mathrm{O}_{2}$. In summary, we hijack a biological process (NETosis) and demonstrate how neutrophils can be used for programmed uptake, transport and delivery of functional nanomaterials.
\end{abstract}




\section{Introduction}

Targeted delivery of (nano)materials and pharmaceuticals is one of the great challenges in biomedicine. ${ }^{1}$ Encapsulation of drug-associated compounds by colloidal structures, such as liposomes or polymeric micelles, have been studied thoroughly over the years and already revealed great success in clinical applications including delivery of active anticancer agents $^{2-4}$, vitamins ${ }^{5}$, enzymes ${ }^{6,7}$ or antimicrobials. ${ }^{8,9}$ Furthermore, nanomaterials such as nanoparticles ${ }^{10-13}$, carbon nanotubes ${ }^{14-16}$ or nanobots ${ }^{17}$ offer additional benefits due to their optoelectronic properties, tunable surface chemistry and ability to infiltrate cellular plasma membranes. ${ }^{14,18}$ Additionally, they can be designed to provide characteristic functions. Single-walled carbon nanotubes (SWCNTs), for example, are known for their near infrared (nIR) fluorescence and their potential to act as a building block for optical nanosensors. ${ }^{19-21}$ With the aid of precise surface modifications, they can be tailored to hold specific detection sites and are able to sense biologically relevant signaling molecules with high spatiotemporal resolution. ${ }^{22,23}$ Thus, such functional objects would be attractive candidates for cargo transport.

On this occasion, a large drawback of conventional drug delivery systems is their incapability to move autonomously. More specifically, many of the materials mentioned above are dependent on an external flow (e.g. of the vascular system) to reach a target zone and as they do not own a particular mechanism to self-propel. Therefore, crossing biological barriers and actively reaching a site of interest remained yet difficult. ${ }^{12,24}$

One way to overcome this issue is to equip the cargo transporter with additional capabilities. Magnetism mediated nanoparticle navigation, for example, was able to demonstrate both, in vitro and in vivo relevance by manipulating carrier trajectories through external force fields ${ }^{25,26}$ It is a powerful tool that, unfortunately, falls off rapidly when associated particles cannot be reached or target zones are not easily locatable. In contrast, turning the body's own cellular response and migration mechanisms into functional transporters seem to become a more convincing trend. ${ }^{27}$ For instance, Din et al. were able to engineer bacteria to perform programmed lysis in vivo resulting in the delivery of cytotoxic agents and a potential way to tackle cancer propagation. ${ }^{28}$ Another example is the binding and transport of cargo molecules by surface-modified red blood cells, which form long-living, biocompatible hybrid carriers and thus enhance the durability and delivery of attached particles. ${ }^{29,30}$ Here, we show that neutrophilic granulocytes (neutrophils), the most abundant type of white blood cell, can be an even more versatile tool to transport functional compounds. Recently, neutrophils received a significant amount of interest due to their attractive, carrier specific properties: they are able to uptake biologic material (phago/endocytosis) $^{31}$, sense and migrate along chemical gradients (chemotaxis) ${ }^{32,33}$ and cross dense borders, such as the blood-brain barrier, by characteristic receptor interactions. ${ }^{34,35}$ In fact, novel camouflage techniques already revealed their capability to incorporate and transport nanomaterials, even though appropriate release mechanisms were still missing to transform these cells into fully operational carrier systems. ${ }^{36}$ For this reason, this study investigated whether neutrophil extracellular trap (NET) formation (NETosis), as their newest anti-inflammatory defense strategy and novel type of cell death, can supplant this role. ${ }^{37,38}$

During NETosis, biochemical signaling pathways force the neutrophil's intracellular chromatin to expand, ultimately leading to the rupture of the cellular membrane and the release of their cytosolic content. Recent investigations of Neubert et al. furthermore showed that this process consists of different phases, including a first active phase in which the cell remains fully functional. ${ }^{39}$ Due to these promising properties, NETosis- 
activated neutrophils could become an excellent tool for cargo uptake and programmed transport and release. Such cells could independently move through biological tissues, follow inflammatory chemical signals and deliver incorporated materials in a NETosismediated fashion. Here, we demonstrate this potential by showing a novel type neutrophil-mediated carrier strategy. We show that neutrophils are able to take up carbonnanotube-based nanosensors as cargo, convey them in vitro and consequently release them by means of NETosis activation. Furthermore, we quantify possible time and length scales in which this new carrier system is able to perform and showcase that the functionality of the internalized material remains preserved over the entire time of delivery.

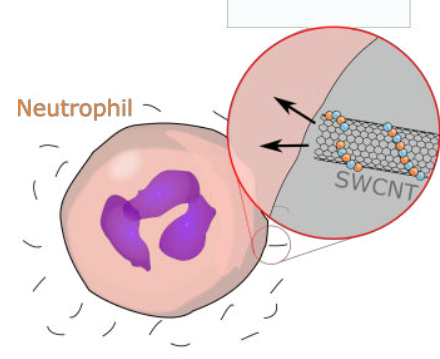

(1) SWCNT Uptake

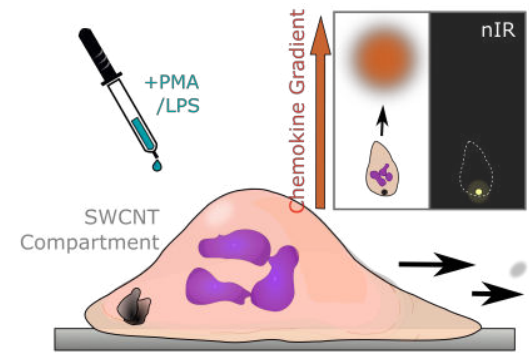

(2) Chemotactic Migration/

Programmed Sensor Transport

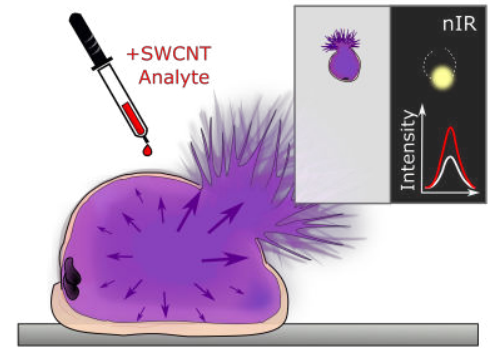

(3) NETotic Cell Rupture/ SWCNT Analyte Contact

Fig. 1: Schematic of uptake, transport and release of cargo by NETotic neutrophils. (1) Neutrophils can take up DNA-coated SWCNT sensors. (2) The neutrophils are still able to migrate and follow an inflammatory signal. At the same time, NETosis is chemically induced which determines how long the cells will migrate and when they perform NETosis. (3) Finally, at the end of the NETotic process, the cellular membrane ruptures and releases the cargo into the extracellular environment. If the load is nanosensors, they can be used in the new location for sensing and imaging applications.

It is well known that neutrophils are able to phagocytose various objects. ${ }^{31}$ In this study case, we choose single-walled carbon nanotubes (SWCNT) as a preferential cargo system since we wanted to make use of their unique fluorescent sensing properties after uptake, transport and release. Interestingly, we were able to ascertain that a simple incubation of neutrophils with DNA functionalized (GT) ${ }_{15}-(6,5)$ SWCNTs leads to a sufficient uptake of the nanoscale material (Fig.2a) and that the cells subsequently transported their cargo in their rear (Fig. 2a, Suppl. Movie 1). Due to this, we speculate that the SWCNTs were internalized into the cell and conveyed to the phagosome, primarily as polarized neutrophils own most of their intracellular compartments at the actomyosin-rich back of the cell. ${ }^{40}$ Similarly, Shi Kam et al. also demonstrated analogical uptake mechanism and locations using streptavidin-functionalized SWCNTs in HL60 cells, a model cell line for primary neutrophils. ${ }^{41}$ The location of the nuclei, on the other side, stayed consistently at the middle/front of the cell, most probably due to its stabilizing and force mediating properties described recently. ${ }^{42}$ We further investigated the uptake dynamics of SWCNTs by a screening assay using different SWCNT concentrations and incubation times $\left(37^{\circ} \mathrm{C}\right.$, in medium). Here, cellular uptake seems to take place within minutes and reaches a saturation point after a 15 - 20-minute mark independent from the amount of exposed material (Fig. 2b). In contrast, the migratory behavior of neutrophils appeared to differ in case of higher SWCNT concentrations. While exposed to lower numbers of functionalized nanotubes $(\approx 0.1 \mathrm{nM})$, cells maintained active and were able to move along independently. Yet, in case of further addition of SWCNTs $(>1 \mathrm{nM})$, we also experienced enhanced cell agglomeration and thus immobility of associated, cumulated neutrophils (Fig. S1a). 
a

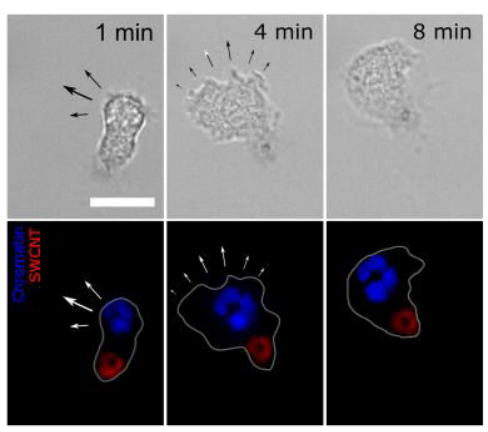

d
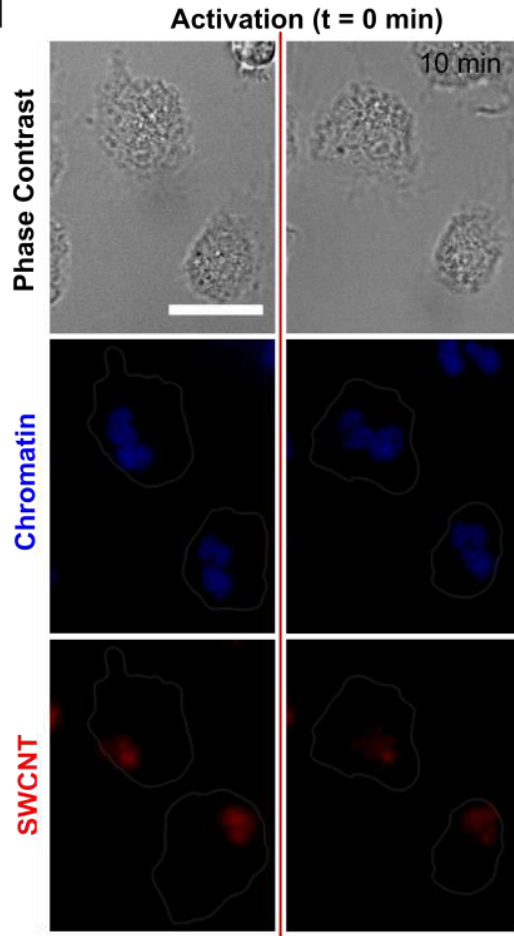

b

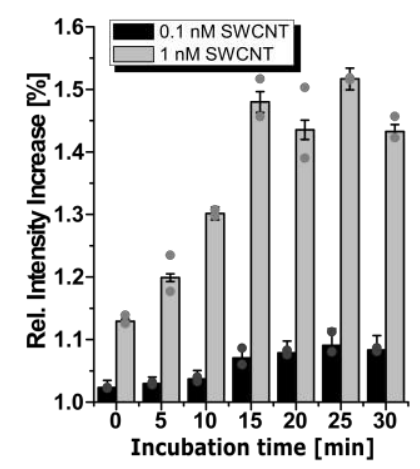

C

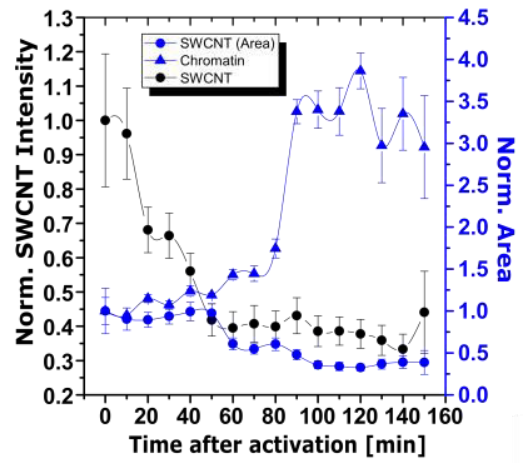

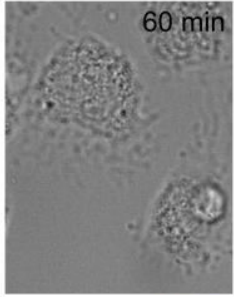
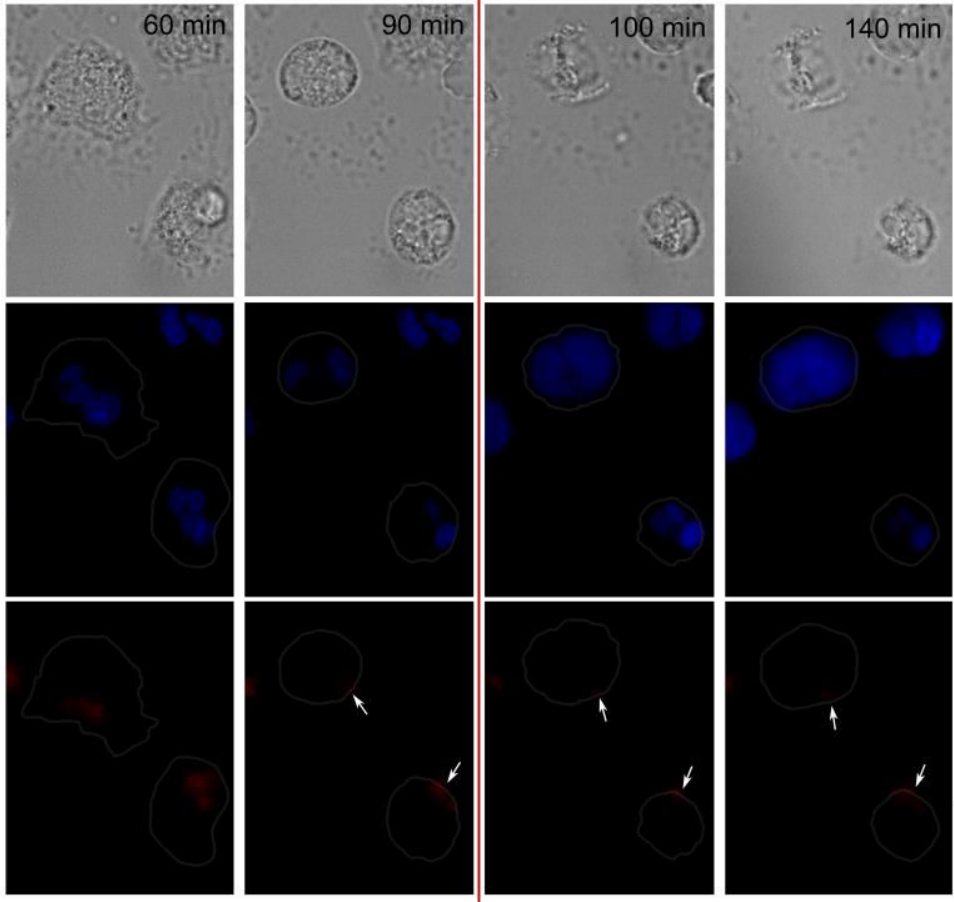

Fig. 2: Uptake of nanomaterial cargo by neutrophils. a Neutrophils take up SWCNT nanosensors and are still able to migrate. Phase contrast (top) and fluorescence images of chromatin (blue, Hoechst 33342 staining) and nIR fluorescent SWCNTs (red). SWCNTs appear to be located at the rear of the cell. b SWCNT uptake kinetics in neutrophils as measured by nIR fluorescence intensity inside the cell. Uptake took place within minutes and saturated after the 15-20 minute mark. Mean $\pm \mathrm{SEM}, \mathrm{N}=2$ donors, $\mathrm{n}>60$ cells. c The SWCNT fluorescence signal changes during NETosis. Both, SWCNT area (blue, circular data points) as well as intensity (black, circular data points) decreased during the process. Here, the latter decreased only while the chromatin area (blue, triangular data points) stayed condensed. Mean \pm SEM, $\mathrm{N}=3$ donors, $\mathrm{n}>30$ cells. d Time course of NETosis in SWCNT loaded and activated neutrophils ((red, bottom), chromatin (blue, middle), phase contrast (top)). Neutrophils showed the common rounding process as well as chromatin decondensation. SWCNTs were pushed by the expanding chromatin to the membrane (arrows).

For this reason, we used $0.1 \mathrm{nM}$ as a preferential SWCNT starting concentration for all proceeding approaches. In these cases, cargo-loaded neutrophils behaved normally and were able to adhere to glass surfaces. Likewise, the cells were still able to undergo NETosis after stimulation with $100 \mathrm{nM}$ PMA and demonstrated the well-documented time course of chromatin decondensation and subsequent cell rupture. Interestingly, the geometry of the corresponding, internalized SWCNT bundle seemed to be affected by the morphological changes of the cell during NET-formation. While the size of the intracellular SWCNT agglomerate did not change in early phases, we experienced a vast compression of these compartments in later stages of NETosis, parallel to the decondensation of the neutrophilic chromatin (Fig. 2c-d, Suppl. Movie 2). A reason for this could be the increasing intracellular pressure which was recently documented to be a consequence of the chromatin swelling. ${ }^{39}$ This would also explain why, most often, SWCNTs appeared to be close to the cellular membrane shortly before cell rupture. Additionally, we found a de- 
crease of the SWCNT's fluorescence intensity within the initial period of NETosis which could be due to increasing levels of MPO/ROS starting to affect the material's optical properties. Here, even though the degradation of SWCNTs by MPO is well known, crucial effects were only reported at different time scales (hours to days). ${ }^{43}$

a

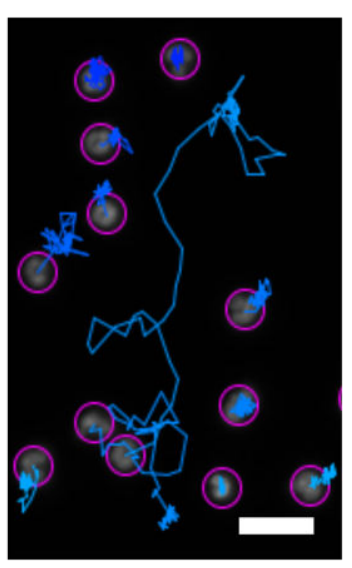

C
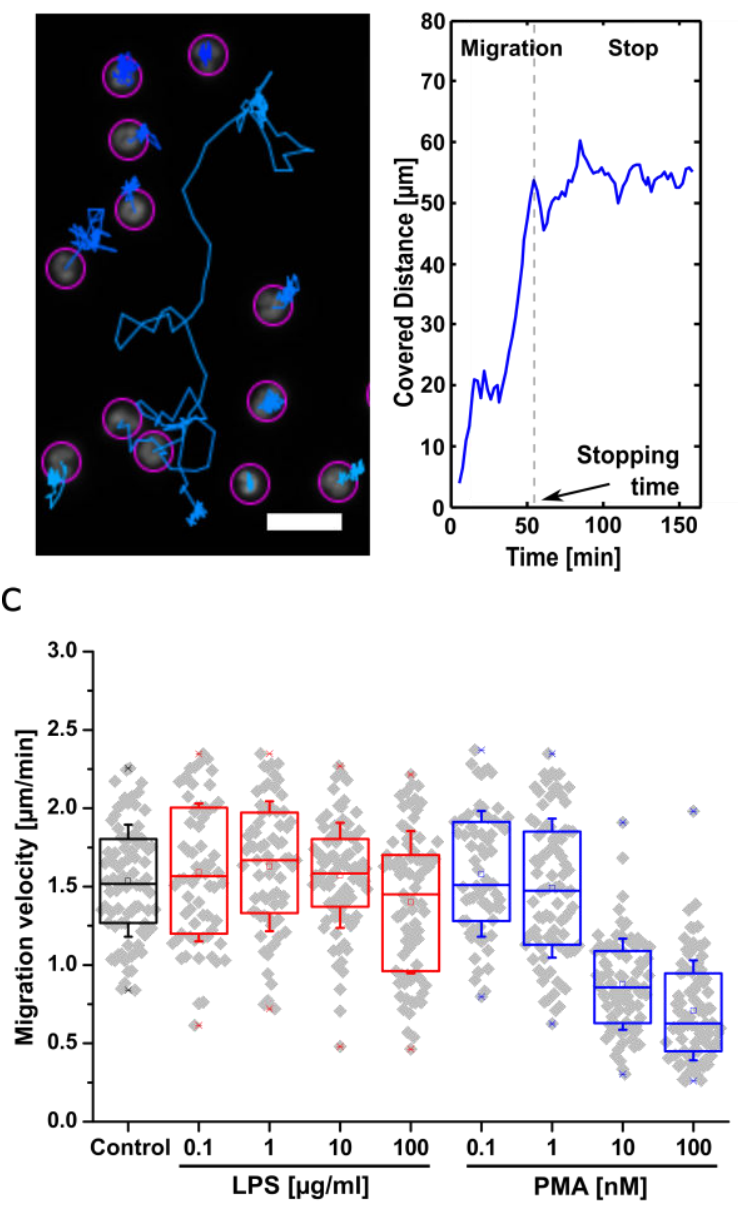

b

d
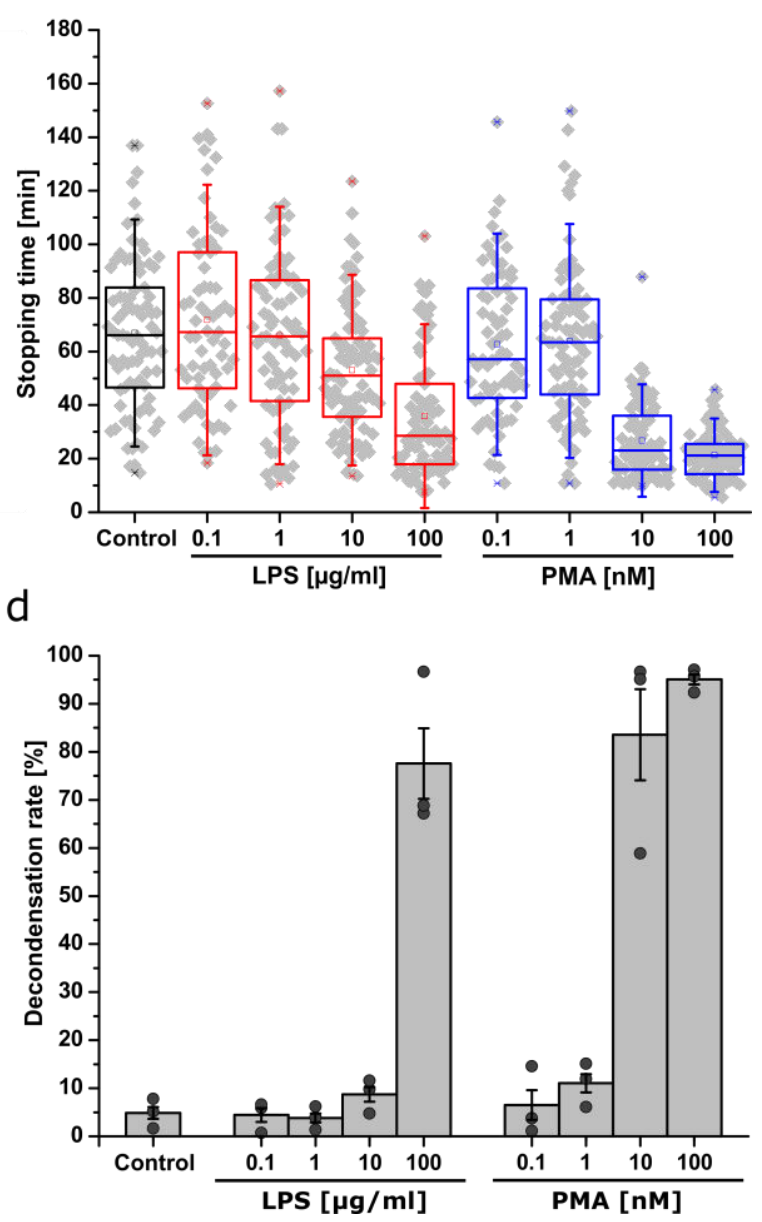

Fig. 3: Migratory properties of activated neutrophils. a Typical trajectories (left) and a corresponding (absolute) covered distance curve of a neutrophil (right). Without inflammatory gradient, cells randomly move in all directions until they stop due to the onset of the second phase of NETosis. Scale bar $=10 \mu \mathrm{m}$. b-c Time until the cells stop to move (stopping time) and migration velocity of activated neutrophils for different activation conditions. Increasing the concentration of LPS (red) increased stopping time linearly. For PMA (blue), there was practically no movement above a certain concentration. Likewise, LPS did not influence the migration velocity whereas PMA slowed them down at higher concentrations. $\mathrm{N}=3$ donors, $\mathrm{n}>60$ cells. Boxplot shows box line $=25-75 \%$ percentile, cross $=$ mean, $\operatorname{dot}=\min / \max$, error bars $=$ SD. d Decondensation (NETosis) rates of neutrophils from b\&c 160 minutes after activation. While very low amounts of LPS and PMA did not influence NETosis activation, higher values $(100 \mu \mathrm{g} / \mathrm{ml} \mathrm{LPS}, 10-100 \mathrm{nM}$ PMA) led to massive chromatin decondensation. Data: mean $+\mathrm{SEM}, \mathrm{N}=3, \mathrm{n}>60$ cells, Nucleus stained with Hoechst 33342 .

On this behalf, the ability of neutrophils to uptake and subsequently undergo NETosis represented the first, necessary step for the development of this new delivery system. However, at this point, it remained elusive if activated, DNA-SWCNT containing cells were still able to migrate or if the activation via specific NETosis inducers would influence their locomotion abilities in a particular manner. Due to this, live-cell imaging of neutrophils exposed to different types and concentrations of NETosis activating compounds (LPS and PMA) were performed in the next step. More specifically, we set neutrophils in environments of distinct activator concentrations and tracked the (random) movement of the most motile cells $(\mathrm{n}=30)$ for each condition and blood donor (Example tracks are shown in Fig. 3a, trajectories for all conditions can be seen in Fig. S2). Here, Fig. 3b-d show the outcome of the associated analysis. We accounted for the average 
stopping time (Fig. 3b), the velocity during the migratory phase (Fig. 3c) and the probability to generate chromatin decondensation (NETosis) after 160 minutes of activation (Fig. 3d, for decondensation analysis, only cells used in $\mathbf{3 b} \& \mathbf{c}$ were counted). The results show that too low activator concentrations $(0.1-10 \mu \mathrm{g} / \mathrm{ml}$ LPS and $0.1-1 \mathrm{nM}$ PMA $)$ did not lead to NETosis yet maintained the neutrophil's motility. On the contrary, too high concentrations $(10,100 \mathrm{nM}$ PMA) resulted in high decondensation rates but also inhibited cell migration completely. Only for median concentrations $(100 \mu \mathrm{g} / \mathrm{ml}$ LPS and $1 \mathrm{nM}$ PMA), cells were still able to migrate while exhibiting a certain probability to exert membrane rupture at the same time (decondensation pictures are shown in Fig. S3). Here, as $1 \mathrm{nM}$ PMA resulted only in a minor number of cell disruptions, we identified 100 $\mu \mathrm{g} / \mathrm{ml}$ LPS as an optimal concentration to guarantee both migration and cargo release via NETosis.

a

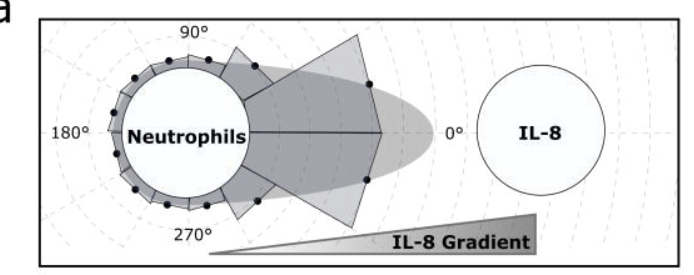

C
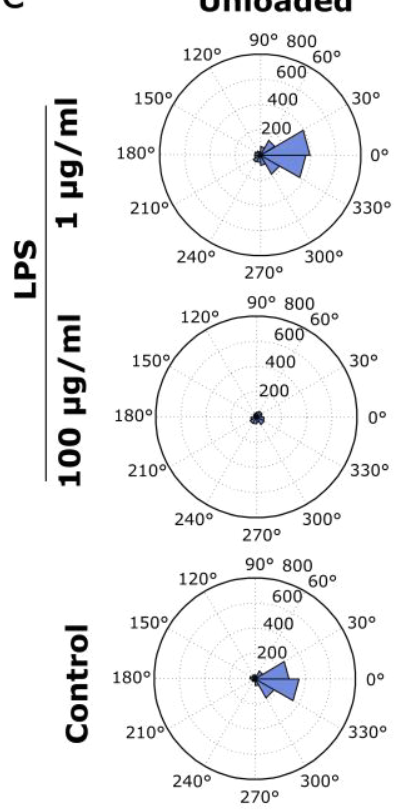

SWCNT
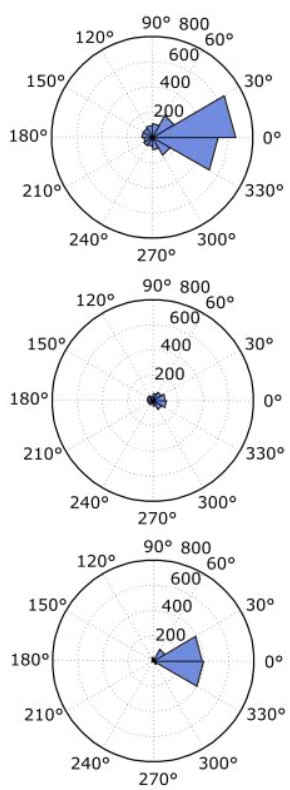
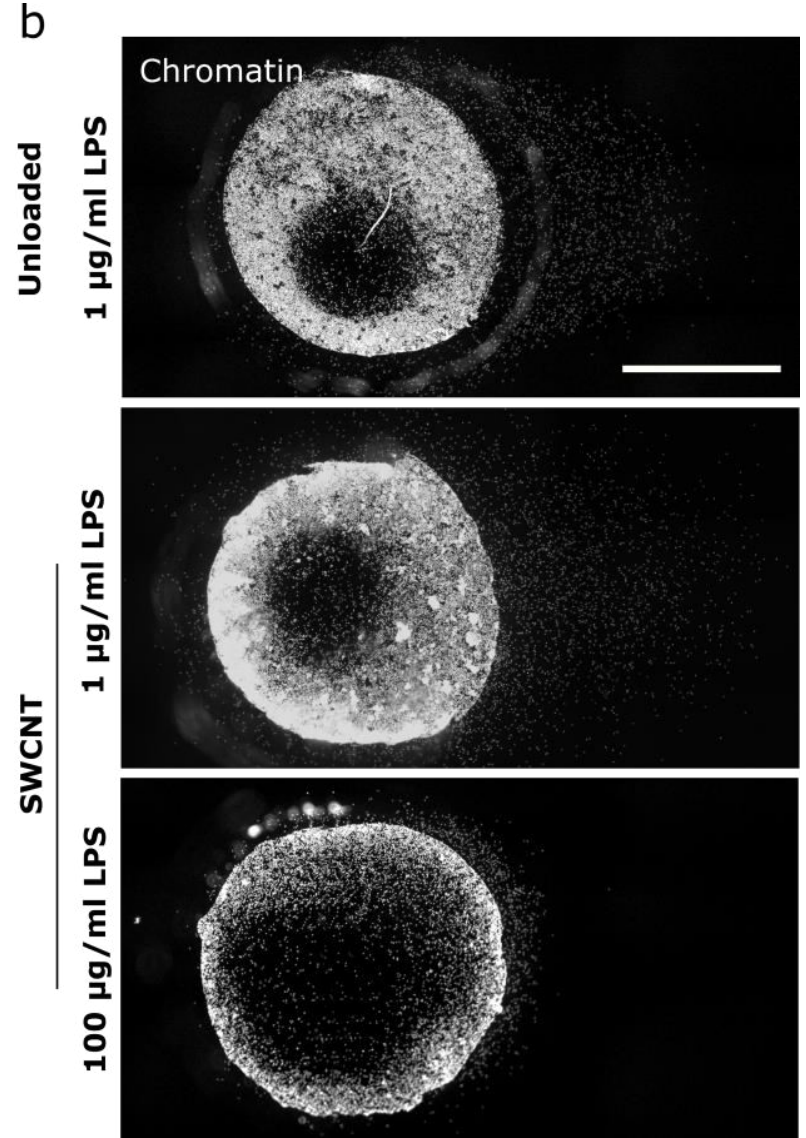

Fig. 4: Collective cargo transport by activated neutrophils. a Design of the migration experiment: Neutrophils, programmed to perform NETosis, migrate along an IL-8 gradient. b The concentration of the NETosis activator affects the period during which the cells still migrate and the onset of NETosis. Images show nuclei of neutrophils (chromatin stained by Hoechst 33342 ) after $3 \mathrm{~h}$ of migration. Scale bar $=500 \mu \mathrm{m}$. c Mean radial migration plots for different activators of NETosis and SWCNT conditions. Higher activator concentrations reduced the migrated distance. Interestingly, SWCNT-loaded cells traveled around $20 \%$ further than the control cells after $3 \mathrm{~h}$

To further investigate whether carrying SWCNTs influence the migration ability of neutrophils, a gradient assay (under-agarose migration) was performed with cargo loaded and unloaded cells. ${ }^{44}$ Both, experiments in high fetal calve serum (FCS) environments $(20 \%$, Fig. S5), as well as commonly used concentrations (0.5\%, Fig. 4, Fig. S4), were accomplished and proved the capability of cells to react to external stimuli (interleukin-8 (IL8) chemokine gradients, Suppl. Movie 3). In addition, to provide comparability with the conditions shown in Fig. 3, the cells were again exposed to varying amounts of activator compounds $(0.1-10 \mathrm{nM}$ PMA \& $1-100 \mu \mathrm{g} / \mathrm{ml}$ LPS $)$ and their chemotactic migra- 
tion distance was quantified after three hours of consecutive movement. Here, similar to the preceding findings, increasing LPS concentrations resulted in less motility/covered distances whereas addition of PMA showed either the same behavior as control samples $(0.1-1 \mathrm{nM})$ or no migration at all $(10 \mathrm{nM})$ implying an unique "all or nothing" behavior of PMA-induced NETosis pathways (Fig. 4c). Furthermore, we could not ascertain significant differences between the migration abilities of SWCNTladen and control cells. Even though we found a slight increase in travel distances in case of SWCNT samples and 0.5\% FCS conditions, these findings reversed when we increased the FCS concentrations (20\%) within the environment.

a

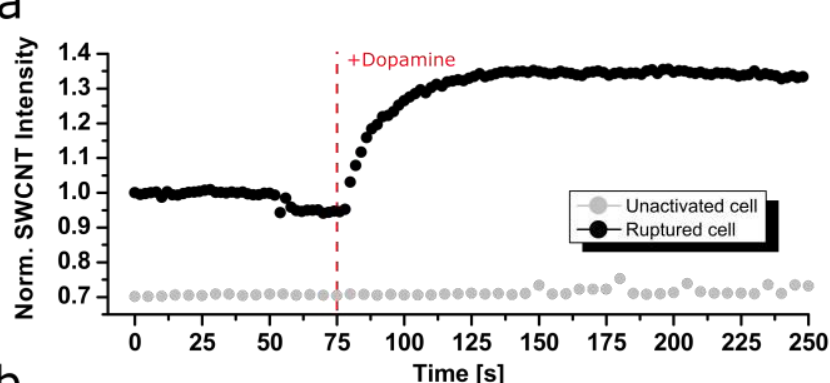

b

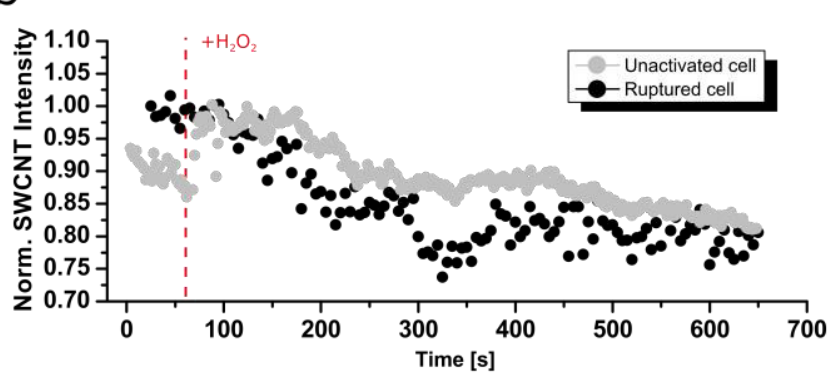

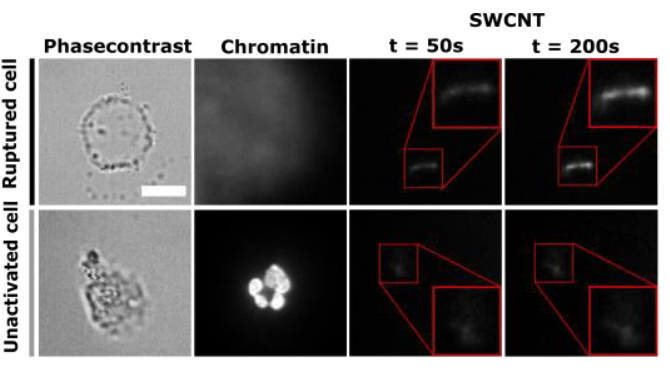

SWCNT

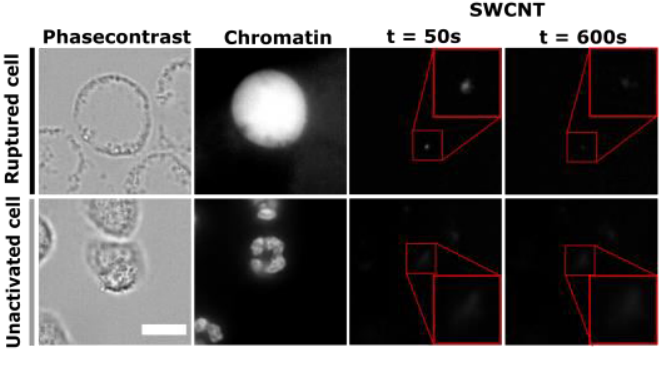

C

Phase Contrast

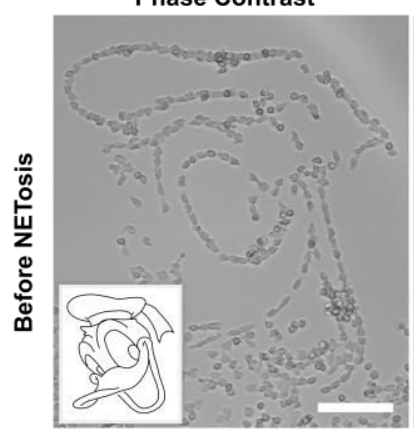

Chromatin d

d SWCNT
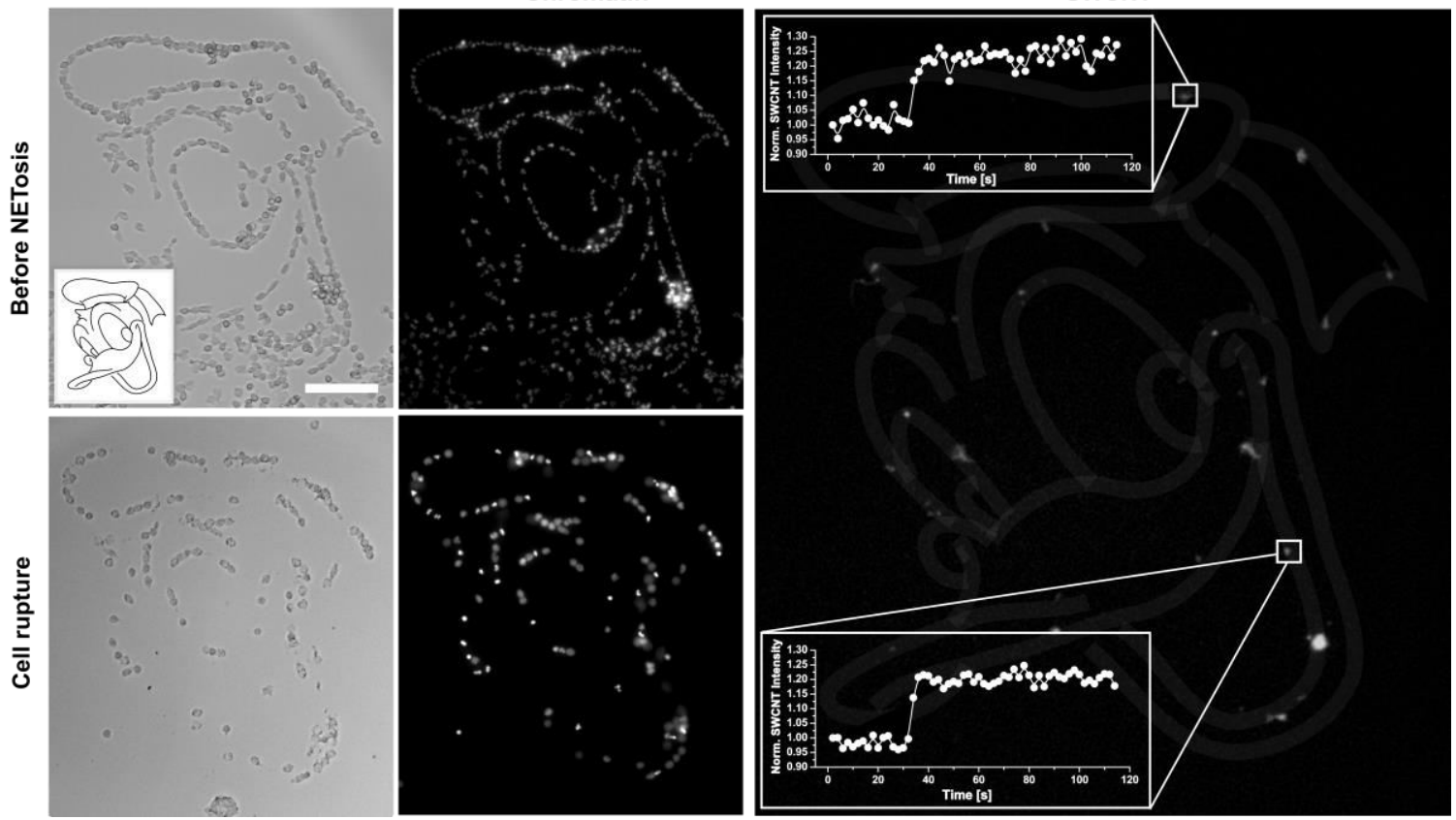
Fig. 5: Release of functional cargo at target sites. a Cells programmed for NETosis release functional cargo such as DNA functionalized SWCNTs that serve as nIR dopamine sensor. (GT) 15 -SWCNTs inside non-activated cells (grey line) showed no changes to 100 $\mathrm{nM}$ dopamine. In contrast, the area around cells that underwent NETosis showed a sensor response (black line) b Other cargo such as hemin-aptamer-SWCNTs respond to $100 \mu \mathrm{M} \mathrm{H} \mathrm{H}_{2}$ both in inactivated (grey) and ruptured cells because $\mathrm{H}_{2} \mathrm{O}_{2}$ diffuses through cells membranes. c Cell patterning of $(\mathrm{GT})_{15}-(6,5)$ SWCNTs loaded neutrophils. Fibrinogen patterns were printed on a glass coverslip to allow neutrophils to migrate to those locations and adhere (top). Activation with $100 \mathrm{nM}$ PMA led to chromatin decondensation of the cells (bottom). d nIR-colocalization of the $(\mathrm{GT})_{15}-(6,5)$ SWCNTs transported by neutrophils. Sensor positions correlated with the pattern (white lines). Sensors were still functional and responded to dopamine $(100 \mathrm{nM})$. Scale bar $=100 \mu \mathrm{m}$, Nucleus staining with Hoechst 33342

In the final step, we also performed functionality tests of the SWCNT cargo in inactivated and ruptured cells to investigate whether the specific abilities of the internalized material remain intact throughout NETosis. Here, as the performance of a given SWCNT compound depends strongly on its surface chemistry, we used $(\mathrm{GT})_{15}-(6,5)$ SWCNTs, known for their ability to sense catecholamines such as dopamine, as well as hemin/aptamer functionalized SWCNTs that were recently found to work as reliable $\mathrm{H}_{2} \mathrm{O}_{2}$ sensors. In both cases, SWCNTs were incorporated by neutrophils and their responses were measured via consecutive nIR imaging either while they were carried within unactivated cells or after NETotic membrane rupture. Here, the addition of $100 \mathrm{nM}$ dopamine into the (GT) ${ }_{15}-(6,5)$ SWCNT samples led to an instantaneous increase of the sensors' intensity in case of disrupted cell membranes, whereas, for undamaged cells, no or only marginal changes could be found (Fig. 5a, Suppl. Movie 4-5). This result indicates a successful release of the cargo after NETotic outbreak and, simultaneously, full functionality of the dopamine nanosensors after release. In contrast, in case of $100 \mu \mathrm{M} \mathrm{H}_{2} \mathrm{O}_{2}$ addition, we experienced in both hemin-aptamer SWCNT samples a steady decrease of the sensors' nIR signal (Fig. 5b, Suppl Movie 6-7), which can be probably explained by the diffusion of $\mathrm{H}_{2} \mathrm{O}_{2}$ through the cellular membrane. ${ }^{45}$ Earlier studies of Ohno et al. further underline this hypothesis as they, likewise, experienced $\mathrm{H}_{2} \mathrm{O}_{2}$ infiltration in neutrophils making it apparent that out hemin-aptamer SWCNTs were able to sense the environmental change even in case of intact cellular membranes. ${ }^{46}$ Interestingly, we were also able to locate differences in the sensors' uptake behavior depending on the associated surface functionalization. While $(\mathrm{GT})_{15}-(6,5)$ SWCNTs appeared most often in larger, intracellular structures, hemin-aptamer SWCNTs were found to be closer to the cellular membrane and defined by smaller agglomeration areas indicating a less efficient internalization of the latter (Fig. 5 a-b, Fig. S6a). Nevertheless, both sensor types appeared to be functional after cargo transport and rupture, predominantly since their response behavior resembled the performance of functionalized SWCNTs prior to cellular uptake (Fig. S6b) and of those used in control experiments (addition of medium, Fig. S6c-d).

Finally, we also demonstrate the transport and release of the functionalized nanosensors to specific target sites. For this purpose, fibrinogen patterns were printed on glass surfaces and SWCNT-laden, activated neutrophils were allowed to migrate over the coated area resulting in an alignment of the cells/sensors due to preferred attachment on the printed protein (Fig. 5c). Again, the immobilized sensors were still functional after cell rupture and showed an instant response to $100 \mathrm{nM}$ dopamine (Fig. 5d, Suppl. Movie 8-9). Thus, this sample case shows that it is possible to let neutrophils uptake nanoscale cargo, transport it to specific sites and release it in a functional state by means of programmed NETosis activation. 


\section{Conclusion}

Over the years much effort has been put into developing biocompatible transport and drug delivery systems. We demonstrated a novel approach that makes use of phagocytosis and migratory behavior of neutrophils and NET formation. As we show, precise chemical activation of neutrophils is able to program how long the cells migrate and the time point of cargo release. In this process, the internalized cargo remains functional at all times and is protected from most extracellular influences as long as the cellular envelope remains intact. This new type of programmable transport-and-release mechanism might be of great benefit for various biomedical applications as it combines the biocompatibility and targeting capabilities of cells with a way to program the time scales of delivery. At the same time, this work also emphasizes the utility of SWCNTs as a beneficial building block for functional materials. In conclusion, we present a novel, multidisciplinary concept for biological carrier development by hijacking and programming immune cells, which holds great potential for directed delivery of functionalized nanomaterials and possible biomedical constituents.

\section{Materials \& Methods}

\section{Cell isolation of human neutrophils}

Isolation of human neutrophils was performed according to the current standard protocols. ${ }^{47}$ Briefly, fresh blood of healthy donors was collected with S-Monovettes KE $7.5 \mathrm{ml}$ (Sarstedt) and layered gently on top of a Histopaque 1119 solution (ratio 1:1). After a first centrifugation step $(1100 \times \mathrm{g}$ for 21 minutes $)$, the transparent third, as well as the colored fourth layer, were collected and mixed with an HBSS (w/o Ca ${ }^{2+} / \mathrm{Mg}^{2+}$, Thermo Fisher Scientific) to ensure a maximal number of neutrophils within the solution. Cells were then again centrifuged for 10 minutes at $400 \times \mathrm{g}$ and the resulting pellet was resuspended in HBSS before layered on top of a phosphate buffered percoll (GE Healthcare) gradient with concentrations of $85,80,75,70$ and $65 \%$. After a third centrifugation $(1100 \times \mathrm{g}, 22$ minutes), neutrophils were extracted by collecting half of the $70 \%$ and $80 \%$ layer as well as the entire $75 \%$ layer and washed once with HBSS. The remaining cell pellet was then resuspended in $1 \mathrm{ml}$ HBSS, counted and lastly diluted to the needed concentration of the experiment. As a culture medium, RPMI 1640 (Lonza) with the addition of $10 \mathrm{mM}$ HEPES and $0.5 \%$ fetal calf serum (FCS, Sigma-Aldrich) was used if not stated otherwise. Cellular identity was furthermore confirmed by a standard cytospin assay (Cytospin 2 Zentrifuge, Shannon) and a subsequent Diff-Quick staining (Medion Diagnostics). Cell purity had to exceed a $95 \%$ threshold to be used for any experiment.

\section{SWCNT modification with ssDNA}

Surface modification of single-walled carbon nanotubes (SWCNTs) was performed as described in Nißler et al. or previous works. ${ }^{48,49}$ Briefly $125 \mu 1 \mathrm{ssDNA}$ solution $(2 \mathrm{mg} / \mathrm{ml}$ stock in phosphate buffered saline (PBS))(Sigma Aldrich) and $125 \mu 1(6,5)$ chirality enriched SWCNTs (Sigma Aldrich, Product No. 773735) $(2 \mathrm{mg} / \mathrm{ml}$ stock in PBS) were placed for tip sonication process (15 min / 30\% Amplitude, Fisher Scientific TM Model 120 Sonic Dismembrator) in an ice bath. The obtained suspension was centrifuged $2 \times 30$ min / RT / $16.100 \mathrm{xg}$, while the AptaCNT solution was furthermore excluded from the excess ssDNA using a Vivaspin 500 MWCO-filter (100.000 Da cut off). The sequence of 
the hemin-binding aptamer was (5'- AGT GTG AAA TAT CTA AAC TAA ATG TGG AGG GTG GGA CGG GAA GAA GTT TAT TTT TCA CAC T-3'). ${ }^{207}$

\section{SWCNT uptake}

To increase absorption rates, around 400000 cells were resuspended in $200 \mu 1$ RPMI 1640 medium in a standard $1.5 \mathrm{ml}$ Eppendorf tube and mixed 1:1 with an SWCNT solution ( $0.2 \mathrm{nM}$ if not stated otherwise), likewise diluted in RPMI 1640 medium. Cells were then incubated at $37{ }^{\circ} \mathrm{C}, 5 \% \mathrm{CO}_{2}$ for 20 minutes, centrifuged once at $600 \times \mathrm{g}$ ( 5 minutes) and washed extensively with RPMI 1640 medium before seeding on the desired substrate.

Absorption analysis, shown in Fig. 1b, was performed equally. Neutrophils were incubated for the stated amount of time, placed in a commonly used $\mu$-slide 8 well chamber slide (ibidi, 75000 cells per well and $200 \mu \mathrm{l}$ RPMI 1640 medium) and fixated using 4\% paraformaldehyde (PFA) (Sigma Aldrich, CAS 30525-89-4) for 1 hour after letting the cells adhere for another 30 minutes at $37{ }^{\circ} \mathrm{C}, 5 \% \mathrm{CO}_{2}$. Fixated samples were then placed in a custom build near-infrared microscope that was composed of a Olympus BX-51 housing (Olympus), regular fluorescence (X-Cite Series 120 Q lamp, EXFO) and white light illumination (TH4-200 lamp, Olympus), a $561 \mathrm{~nm}$ laser (Jive 500, Cobolt) and two nIR perceptive cameras (Zyla 4.2 sCMOS, Andor and Xeva-1.7-320, Xenics) with a $900 \mathrm{~nm}$ long pass filter FEL0900, Thorlabs) in front to visualize SWCNT excitation as well as phase contrast and fluorescence staining in parallel. For imaging, a 20x objective (MPLFLN20X, Olympus) was chosen to increase the observable cell and SWCNT number. For each condition, four images at each side of a well were recorded in phase contrast and nIR-mode (100 $\mathrm{mW}$ laser power, $500 \mathrm{~ms}$ exposure time) using the Zyla camera and the last mentioned objective and all images were saved in separate 16-bit files for subsequent analysis.

Measuring the intensity of SWCNT inside the cells was then performed using ImageJ's thresholding system (v3.52i). nIR images were tuned to 8-bit depths, cropped into 20x20 $\mu \mathrm{m}$ areas containing only the respective SWCNTs and thresholded via common MinError thresholding algorithm to calculate a mean intensity. SWCNT spots that couldn not be correlated with a cell area in the corresponding phase contrast image, as well as sensors that were found in cell agglomerations, were excluded from the analysis. The remaining data were averaged (weighted mean of all experiments) over all images and normalized over the corresponding background value $\left(\mathrm{I}_{\mathrm{br}}=1\right)$ to calculate a normalized mean intensity for each condition.

\section{Live cell imaging of SWCNTs during NETosis}

$1 \mathrm{ml}$ of RPMI medium containing $200000(\mathrm{GT})_{15}-(6,5)$ SWCNTs loaded cells and a 1 $\mu \mathrm{g} / \mathrm{ml}$ concentration of Hoechst 33342 (Cat. H1399, Thermo Fisher Scientific) was poured on a glass bottom Petri Dish (Cat. 150680, Thermo Fisher Scientific) and placed in an incubator system $\left(37^{\circ} \mathrm{C}, 5 \% \mathrm{CO}_{2}\right)$ for 30 minutes to enable sufficient adhesion.

Subsequently, the sample was placed into a preheated incubation system $\left(37^{\circ} \mathrm{C}\right)$ (Cat. 11922 , ibidi) which sat on top of the custom build near-infrared microscope mentioned in the previous section. A 100x oil objective (UPLSAPO 100XO, Olympus) was slowly approached to the sample until a sharp picture could be seen within all channels. Consecutively, phase contrast, as well as chromatin (DAPI) and nIR-images, were taken manually from the chosen sample position every ten minutes for 150 minutes after addition of 1 $\mathrm{ml}$ of RPMI medium that contained $200 \mathrm{nM}$ of phorbol myristate acetate (PMA) (SigmaAldrich). SWCNTs were excited by a common fluorescence lamp in combination with a 
built-in $561 \mathrm{~nm}$ filter cube (F48-553, AHF Analysentechnik) and excitation powers and exposure times were kept constant over the entire experiment to ensure comparability within the entire data set.

Analysis of chromatin and SWCNT area as well as SWCNT intensity was similar to the absorption study. Images were transformed into 8-bit pictures, cropped in small 20x20 $\mu \mathrm{m}$ sub-images that contained only the sought excitation area and, if necessary, their background was equalized to ensure comparability between each frame. Likewise, ImageJ's MinError thresholding algorithm was used to measure all needed parameters and, after calculation of the weighted mean of each experiment and frame, all data sets were normalized to the value gained at $\mathrm{t}=0 \mathrm{~s}$.

\section{Live cell imaging of activated neutrophil migration}

To record the migration behavior of activated neutrophils, around $200 \mu \mathrm{l}$ of RPMI 1640 medium containing 75000 untreated cells and $1 \mu \mathrm{g} / \mathrm{ml}$ Hoechst 33342 stain was placed in nine different wells of common 8 -well $\mu$-slides (ibidi) and was incubated for 20 minutes at $37{ }^{\circ} \mathrm{C}$ and $5 \% \mathrm{CO}_{2}$. Subsequently, the $\mu$-slides were incorporated in pre-heated ibidi heating chambers $\left(37^{\circ} \mathrm{C}, 5 \% \mathrm{CO}_{2}, 90 \%\right.$ humidity) that were set on top of Olympus IX-81 microscopes. Using integrated XY-stages, 20x objectives (UCPLFLN 20x, Olympus) and corresponding phase contrast and DAPI channels (CBH white light lamp, U-HGLGPS fluorescence lamp, Olympus and 86-370-OLY DAPI filter-cube), a suitable position within each well was chosen and saved using the implemented steering software (Cell Sense, v. 2.1, Olympus). Subsequently, $200 \mu 1$ of RPMI medium containing a distinct concentration of PMA $(0.2,2,20,200 \mathrm{nM})$ or lipopolysaccharide from Pseudomonas Aeruginosa (LPS) $(0.2,2,20,200 \mu \mathrm{g} / \mathrm{ml})$ (Sigma-Aldrich) were added to a well in a blinded fashion and phase contrast as well as DAPI channels were recorded every two minutes for 160 minutes to track cell movement at each chosen position. Lastly, all images were exported as 8-bit TIFF stacks for further analysis.

\section{Migration analysis of preactivated neutrophils}

Cell tracking was then performed by ImageJ's TrackMate plugin which ensured efficient segmentation and position determination for the entire analysis time..$^{50}$ Briefly, all chromatin images gained by the process mentioned above were combined to form a z-stack and implemented into the plugin without further treatment. Segmentation was then performed using the Laplacian of Gaussian (LoG) detector module with an estimated blob diameter of 20 pixels $(6.5 \mu \mathrm{m})$ and a 5 pixel $(1.6 \mu \mathrm{m})$ threshold. As a result, we were able to trace back all cell trajectories within a stack by using the subsequent simple LAP tracker model with a maximal linking and gap-closing distance of 50 pixels $(16 \mu \mathrm{m})$ as well as a maximal frame gap within a trajectory of two frames. This adjustment, in particular, enabled accurate tracking of migrating cells while cell bodies that just floated around were neglected by the algorithm. All trajectories were then further analyzed by a custom-build MATLAB code (v. Matlab 2014a) which was able to calculate the traveled distance for every cell and frame i according to the formula

$$
d\left(t_{i}\right)=\left|\left(\begin{array}{l}
x\left(t_{i}\right) \\
y\left(t_{i}\right)
\end{array}\right)-\left(\begin{array}{l}
x(0) \\
y(0)
\end{array}\right)\right|=\sqrt{\left(x\left(t_{i}\right)-x(0)\right)^{2}+\left(y\left(t_{i}\right)-y(0)\right)^{2}}
$$

Subsequently, for each condition, 30 cells with the highest average distance values of the data set were extracted and further analyzed to detect the migration behavior of the most 
motile neutrophils within the environment. The stopping time of the migratory phase was calculated by plotting the distance-plots, likewise in a blinded fashion, together with the respective cell trajectory and manually searching for a time point of stagnation within the data set for each cell. Cells that did not show a clear change of moving patterns were excluded from the data set. However, cells with consistent migration abilities over 160 minutes could not be found. Cell velocity of the migratory phase was then simply calculated by averaging the cell speed

$$
\bar{v}=\frac{1}{(N-1) \tau} \sum_{i=2}^{N} \sqrt{\left(y\left(t_{i}\right)-y\left(t_{i-1}\right)\right)^{2}+\left(x\left(t_{i}\right)-x\left(t_{i-1}\right)\right)^{2}}
$$

with $\mathrm{N}$ defining the number of frames until the stopping time and $\tau$ depict the frame time ( 2 minutes) between two images.

\section{Decondensation rate analysis}

Counting decondensed and intact/lobular shaped nuclei was performed according to already existing protocols. ${ }^{39,51}$ Briefly, chromatin images of the recorded positions were taken after 180 minutes and analyzed via ImageJ's Cell Counter plugin. Nuclei that appeared in its known, compressed shape were counted and defined as intact/condensed whereas nuclei that showed increased, roundish chromatin distributions defined a basis for the decondensed/NETotic state. Cells were counted and the number of decondensed cells was divided by the total number of cells to generate a relative decondensation value.

\section{Under agarose assay - Gel casting}

Under agarose assays were performed to measure cell migration of SWCNT-loaded neutrophils due to their simple and yet high producible properties. Gels were manufactured according to the protocol of B. Heit et al.. ${ }^{44}$ A HBSS/RPMI 1640 solution was prepared by mixing $5 \mathrm{ml} \mathrm{HBSS} \mathrm{(w/o} \mathrm{Ca}{ }^{2+}, \mathrm{Mg}^{2+}$, Thermo Fisher Scientific) and $10 \mathrm{ml}$ RPMI containing $0.75 \%$ FCS (Merck) in a standard 50-ml Eppendorf tube and heated up to $68^{\circ} \mathrm{C}$ using a conventional water bath. Meanwhile, $0.24 \mathrm{~g}$ ultra-pure agarose (Roth) was added to a vial containing $5 \mathrm{ml}$ of milliQ and the solution was vortexed extensively in order to suspend the agarose homogeneously. The latter was subsequently heated up until boiling by the use of a common Bunsen burner and quickly vortexed for three times. The HBSS/RPMI solution was added to the agarose and $3 \mathrm{ml}$ each of the mixture was evenly distributed on a plastic-bottom petri dish (Cat. 81156, ibidi). Agarose gels were then allowed to solidify at room temperature and samples were stored overnight at $4{ }^{\circ} \mathrm{C}$ with the dish lid covered in dust-free, milliQ saturated cloths to avoid gel draining. Shortly before the cell experiment, two wells with a diameter of $3 \mathrm{~mm}$ and a distance of $2.2 \mathrm{~mm}$ were punched in each gel using a dermal biopsy punch (Cat. KBP-48101, kai medical) and remaining agarose within each of the wells was extensively removed by vacuum aspiration. Lastly, gels were equilibrated with RPMI 1640 medium for one hour $\left(37^{\circ} \mathrm{C}, 5 \%\right.$ $\mathrm{CO}_{2}$ ) and the supernatant medium was again removed by vacuum aspiration shortly before cell loading. 


\section{Under agarose assay - cell loading}

Around 100000 cells, pre-equilibrated for 20 minutes in a (GT) ${ }_{15}-(6,5)$ SWCNTs solution or without any pre-treatment, were poured in one of the prepared agarose wells using $5 \mu 1$ of medium with an addendum of $1.6 \mu \mathrm{M}$ Hoechst 33342 and were allowed to equilibrate for 20 minutes at $37{ }^{\circ} \mathrm{C}, 5 \% \mathrm{CO}_{2}$. Subsequently, $10 \mu \mathrm{l}$ of a $0.1 \mu \mathrm{M}$ IL-8 solution was poured into the second well while $5 \mu \mathrm{l}$ of the respective NETosis activator concentration (LPS: $200,20,2 \mu \mathrm{g} / \mathrm{ml}$, PMA: $20,2,0.2 \mathrm{nM}$ or medium as control) was added to the cell well in the same time without causing any kind of bubble formation. All samples were stored for three hours in a commonly used incubator $\left(37{ }^{\circ} \mathrm{C}, 5 \% \mathrm{CO}_{2}\right)$ and imaged afterward using the aforementioned IX-81 microscopic setup in combination with a $4 \mathrm{x}$ objective (UPLFLN 4X, Olympus). Afterward, cells were fixated by adding 2\% PFA in PBS after carefully removing the gels with a scalpel and tweezers, washed twice with PBS, stored at $4{ }^{\circ} \mathrm{C}$ overnight and nIR-imaged by the aid of our aforementioned, customized nIR-setup the next day.

\section{Under agarose assay - migration analysis}

For image analysis, single images of each sample were stitched using ImageJ's plugin MosaicJ which enabled an accurate composition due to the build in manual alignment tool. Fused images were background corrected and the existing cell distribution outside of the well was divided radially into 30-degree sections (see Fig. S5) in which the distance of the leading cell edge was manually analyzed using ImageJ's line tool. Resulting distances were averaged over all experiments and plotted as radial bar plots shown in Fig. 4 or Fig. S4-5.

\section{Sensor functionality tests}

Cells for SWCNT functionality tests were prepared equally to those used for live cell imaging: neutrophils were priorly incubated in a $0.1 \mathrm{nM}$ SWCNT solution in medium for 20 minutes and seeded on a glass bottom petri dish by pouring $1 \mathrm{ml}$ of medium (containing around 200000 cells and $1.6 \mu \mathrm{M}$ of additional Hoechst 33342) in it. Cells that should show a ruptured state were confronted with $100 \mathrm{nM}$ PMA (1:1 mixing of $200 \mathrm{nM}$ PMA in medium) after 30 minutes of seeding time and were incubated for three hours before imaging.

Recording of the sensor response in intact or ruptured neutrophils was again performed at the customized build nIR-setup mentioned above in combination with an integrated ibidi heating chamber $\left(37^{\circ} \mathrm{C}\right)$ and the 100x oil objective. SWCNT of each condition were excited by a $561 \mathrm{~nm}$ laser $(200 \mathrm{~mW})$ and recorded with a frame time of 2 or 5 seconds per image for four (dopamine addendum) or respectively 10 minutes $\left(\mathrm{H}_{2} \mathrm{O}_{2}\right.$ addendum) in which, around the 1 minute mark, $500 \mu l$ of the particular analyte ( $300 \mathrm{nM}$ dopamine, 300 $\mu \mathrm{M} \mathrm{H}_{2} \mathrm{O}_{2}$, both in medium) was added. Subsequent exportation of image files and intensity analysis of single SWCNT areas were performed as described in the live cell imaging.

\section{Cell patterning}

Forming cell patterns on a substrate was realized with light-induced fibrinogen printing controlled by a PRIMO micropatterning machine (alvéole). Briefly, 18 x $18 \mathrm{~mm}$ glass coverslips (Fisher Scientific) were washed with $75 \%$ ethanol twice and plasma treated for 
five minutes to clean and improve the hydrophilicity of the sample. Subsequently, PDMS stencils with a circular well $(\mathrm{r}=2 \mathrm{~mm})$ in the center were pressed onto the glass and filled with $0.1 \mathrm{mg} / \mathrm{ml}$ PLL-g-PEG (Sigma) in PBS for one hour to guarantee a homogeneous passivation layer. Next, the so prepared sample was fixated on the respective PRIMO setup, calibrated according to the manufacturers' instructions (microscope specs: Olympus IX83 with an UCPlanFL N 20x objective and an IX3-SSU stage) and filled with $5 \mu 1$ of the photoactivable reagent PLPP (1x, alvéole). Local radiation of the PLPP bound PEG-substrate using the patterning software LEONARDO (alvéole) then led to a protein degradation within the illuminated spots and enabled deposition of a second substrate protein. Due to this, the sample was washed three times with PBS, coated again with Alexa-488 labeled fibrinogen solution (10 $\mu \mathrm{l}$ of a $50 \mu \mathrm{g} / \mathrm{ml}$ solution in PBS, Sigma) for twenty minutes and stored at $4{ }^{\circ} \mathrm{C}$ after another three washing steps. Patterns were normally used the next day,however, pattern degradation was not visible within one week after printing.

\section{Acknowledgments}

This project was supported by the state of Lower Saxony (life@nano) and the German Research Foundation (DFG grant KR 4242/4-1 and ER 723/2-1). We thank Andreas Janshoff and Claudia Steinem for fruitful discussions and support. We are grateful for fruitful discussions about active matter with members of the collaborative research center SFB 937 funded by the DFG. We thank the IMPRS PCBS for a PhD student fellowship (D.M.).

\section{References}

1. Peer, D. et al. Nanocarriers as an emerging platform for cancer therapy. Nat. Nanotechnol. 2, 751-760 (2007).

2. Allen, T. M. \& Cullis, P. R. Liposomal drug delivery systems: From concept to clinical applications. Adv. Drug Deliv. Rev. 65, 36-48 (2013).

3. Sarris, A. H. et al. Liposomal vincristine in relapsed non-Hodgkin's lymphomas: early results of an ongoing phase II trial. Ann. Oncol. Off. J. Eur. Soc. Med. Oncol. 11, 69-72 (2000).

4. Nakanishi, T. et al. Development of the polymer micelle carrier system for doxorubicin. J. Control. Release 74, 295-302 (2001).

5. Mohammadi, M., Ghanbarzadeh, B. \& Hamishehkar, H. Formulation of nanoliposomal vitamin d3 for potential application in beverage fortification. $A d v$. Pharm. Bull. 4, 569-75 (2014).

6. Ulbrich, R., Golbik, R. \& Schellenberger, A. Protein adsorption and leakage in carrier-enzyme systems. Biotechnol. Bioeng. 37, 280-287 (1991).

7. Torchilin, V. P., Tischenko, E. G., Smirnov, V. N. \& Chazov, E. I. Immobilization of enzymes on slowly soluble carriers. J. Biomed. Mater. Res. 11, 223-235 (1977).

8. Emami, S., Azadmard-Damirchi, S., Peighambardoust, S. H., Valizadeh, H. \& Hesari, J. Liposomes as carrier vehicles for functional compounds in food sector. $J$. Exp. Nanosci. 11, 737-759 (2016).

9. Tiwari, G. et al. Drug delivery systems: An updated review. Int. J. Pharm. 
Investig. 2, 2-11 (2012).

10. Hirsch, L. R. et al. Nanoshell-mediated near-infrared thermal therapy of tumors under magnetic resonance guidance. Proc. Natl. Acad. Sci. U. S. A. 100, 13549-54 (2003).

11. Jain, S., Hirst, D. G. \& O'Sullivan, J. M. Gold nanoparticles as novel agents for cancer therapy. Br. J. Radiol. 85, 101-13 (2012).

12. De Jong, W. H. \& Borm, P. J. A. Drug delivery and nanoparticles:applications and hazards. Int. J. Nanomedicine 3, 133-49 (2008).

13. Wang, Y.-F., Liu, L., Xue, X. \& Liang, X.-J. Nanoparticle-based drug delivery systems: What can they really do in vivo? F1000Research 6, 681 (2017).

14. Bianco, A., Kostarelos, K. \& Prato, M. Applications of carbon nanotubes in drug delivery. Curr. Opin. Chem. Biol. 9, 674-679 (2005).

15. Guo, Q., Shen, X., Li, Y. \& Xu, S. Carbon nanotubes-based drug delivery to cancer and brain. Curr. Med. Sci. 37, 635-641 (2017).

16. Zhang, W., Zhang, Z. \& Zhang, Y. The application of carbon nanotubes in target drug delivery systems for cancer therapies. Nanoscale Res. Lett. 6, 555 (2011).

17. Saadeh, Y. \& Vyas, D. Nanorobotic Applications in Medicine: Current Proposals and Designs. Am. J. Robot. Surg. 1, 4-11 (2014).

18. Patra, J. K. et al. Nano based drug delivery systems: recent developments and future prospects. J. Nanobiotechnology 16, 71 (2018).

19. Mann, F. A. F. A., Herrmann, N., Meyer, D. \& Kruss, S. Tuning selectivity of fluorescent carbon nanotube-based neurotransmitter sensors. Sensors (Switzerland) 17, (2017).

20. Mann, F. A. et al. Carbon Nanotubes Encapsulated in Coiled-Coil Peptide Barrels. Chem. - A Eur. J. 24, 12241-12245 (2018).

21. Polo, E. et al. Control of Integrin Affinity by Confining RGD Peptides on Fluorescent Carbon Nanotubes. ACS Appl. Mater. Interfaces 10, 17693-17703 (2018).

22. Kruss, S. et al. High-resolution imaging of cellular dopamine efflux using a fluorescent nanosensor array. Proc. Natl. Acad. Sci. U. S. A. 114, 1789-1794 (2017).

23. Meyer, D., Hagemann, A. \& Kruss, S. Kinetic Requirements for Spatiotemporal Chemical Imaging with Fluorescent Nanosensors. ACS Nano 11, 4017-4027 (2017).

24. Anselmo, A. C. Cell-mediated delivery of nanoparticles: Taking advantage of circulatory cells to target nanoparticles. J. Control. Release 190, 531-541 (2014).

25. Berry, C. C., Wells, S., Charles, S. \& Curtis, A. S. G. Dextran and albumin derivatised iron oxide nanoparticles: influence on fibroblasts in vitro. Biomaterials 24, 4551-7 (2003).

26. A multicenter, phase I/II trial of hepatic intra-arterial delivery of doxorubicin hydrochloride adsorbed to magnetic targeted carriers in patients with hepatocellular carcinoma. Eur. J. Cancer 38, S18 (2002). 
27. Agrahari, V., Agrahari, V. \& Mitra, A. K. Next generation drug delivery: circulatory cells-mediated nanotherapeutic approaches. Expert Opin. Drug Deliv. 14, 285-289 (2017).

28. Din, M. O. et al. Synchronized cycles of bacterial lysis for in vivo delivery. Nature 536, 81-85 (2016).

29. Villa, C. H., Anselmo, A. C., Mitragotri, S. \& Muzykantov, V. Red blood cells: Supercarriers for drugs, biologicals, and nanoparticles and inspiration for advanced delivery systems. Adv. Drug Deliv. Rev. 106, 88-103 (2016).

30. Shi, J. et al. Engineered red blood cells as carriers for systemic delivery of a wide array of functional probes. Proc. Natl. Acad. Sci. U. S. A. 111, 10131-6 (2014).

31. Gustafson, H. H., Holt-Casper, D., Grainger, D. W. \& Ghandehari, H. Nanoparticle Uptake: The Phagocyte Problem. Nano Today 10, 487-510 (2015).

32. David, R. How neutrophils set their compass. Nat. Rev. Mol. Cell Biol. 14, 547547 (2013).

33. Wang, F. The signaling mechanisms underlying cell polarity and chemotaxis. Cold Spring Harb. Perspect. Biol. 1, a002980 (2009).

34. Xue, J. et al. Neutrophil-mediated anticancer drug delivery for suppression of postoperative malignant glioma recurrence. Nat. Nanotechnol. 12, 692-700 (2017).

35. Chu, D., Dong, X., Shi, X., Zhang, C. \& Wang, Z. Neutrophil-Based Drug Delivery Systems. Adv. Mater. 30, 1706245 (2018).

36. Shao, J. et al. Chemotaxis-Guided Hybrid Neutrophil Micromotors for Targeted Drug Transport. Angew. Chemie Int. Ed. 56, 12935-12939 (2017).

37. V., B. et al. Neutrophil Extracellular Traps Kill Bacteria. Science (80-. ). 303, 1532-1535 (2004).

38. Fuchs, T. A. et al. Novel cell death program leads to neutrophil extracellular traps. J. Cell Biol. 176, 231-41 (2007).

39. Neubert, E. et al. Chromatin swelling drives neutrophil extracellular trap release. Nat. Commun. 9, 3767 (2018).

40. Mitchell, T., Lo, A., Logan, M. R., Lacy, P. \& Eitzen, G. Primary granule exocytosis in human neutrophils is regulated by Rac-dependent actin remodeling. Am. J. Physiol. Cell Physiol. 295, C1354-65 (2008).

41. Kam, N. W. S., Jessop, T. C., Wender, P. A. \& Dai, H. Nanotube molecular transporters: Internalization of carbon nanotube-protein conjugates into mammalian cells. J. Am. Chem. Soc. (2004). doi:10.1021/ja0486059

42. Manley, H. R., Keightley, M. C. \& Lieschke, G. J. The Neutrophil Nucleus: An Important Influence on Neutrophil Migration and Function. Front. Immunol. 9, 2867 (2018).

43. Vlasova, I. I., Sokolov, A. V., Chekanov, A. V., Kostevich, V. A. \& Vasilyev, V. B. Myeloperoxidase-induced biodegradation of single-walled carbon nanotubes is mediated by hypochlorite. Russ. J. Bioorganic Chem. 37, 453-463 (2011).

44. Heit, B. \& Kubes, P. Measuring Chemotaxis and Chemokinesis: The UnderAgarose Cell Migration Assay. Sci. Signal. (2003). doi:10.1126/stke.2003.170.p15 
45. Kim, J.-H. et al. Single-Molecule Detection of $\mathrm{H}_{2} \mathrm{O}_{2}$ Mediating Angiogenic Redox Signaling on Fluorescent Single-Walled Carbon Nanotube Array. ACS Nano 5, 7848-7857 (2011).

46. Ohno, Y. \& Gallin, J. I. Diffusion of extracellular hydrogen peroxide into intracellular compartments of human neutrophils: Studies utilizing the inactivation of myeloperoxidase by hydrogen peroxide and azide. J. Biol. Chem. (1985).

47. Mantovani, A., Cassatella, M. A., Costantini, C. \& Jaillon, S. Neutrophils in the activation and regulation of innate and adaptive immunity. Nature Reviews Immunology (2011). doi:10.1038/nri3024

48. Kruss, S. et al. Neurotransmitter detection using corona phase molecular recognition on fluorescent single-walled carbon nanotube sensors. J. Am. Chem. Soc. (2014). doi:10.1021/ja410433b

49. Kruss, S. et al. Quantification of the Number of Adsorbed DNA Molecules on Single-Walled Carbon Nanotubes. J. Phys. Chem. C (2019). doi:10.1021/acs.jpcc.8b11058

50. Tinevez, J. Y. et al. TrackMate: An open and extensible platform for singleparticle tracking. Methods (2017). doi:10.1016/j.ymeth.2016.09.016

51. Wong, S. L. et al. Diabetes primes neutrophils to undergo NETosis, which impairs wound healing. Nat. Med. (2015). doi:10.1038/nm.3887 


\section{Supplementary Informations}

Programmed transport and release of nanoscale cargo by immune cells

Daniel Meyer ${ }^{1}$, Saba Telele ${ }^{1}$, Anna Zelená ${ }^{3}$, Elsa Neubert ${ }^{2}$, Robert Nissler ${ }^{1}$, Florian Mann $^{1}$, Julia Grandke ${ }^{2}$, Luise Erpenbeck ${ }^{2}$, Sarah Köster ${ }^{3}$, Sebastian Kruss ${ }^{1 *}$

${ }^{1}$ Institute of Physical Chemistry, Göttingen University, Germany

${ }^{2}$ Department of Dermatology, University Medical Center, Göttingen University, Germany

${ }^{3}$ Institute of X-Ray Physics, Göttingen University, Germany

${ }^{+}$Correspondence should be addressed to Sebastian Kruss (S.K., email: skruss@unigoettingen.de)

\section{Supplemental movies}

Suppl. Movie 1: nIR movie of SWCNT loaded neutrophils migrating in a $100 \mathrm{nM}$ fMLP treated environment

Suppl. Movie 2: Multichannel live-cell images of PMA activated neutrophils (Phase Contrast, Chromatin, (GT) ${ }_{15}$-SWCNT)

Suppl. Movie 3: Under-agarose neutrophil migration, medium contained $1 \mu \mathrm{g} / \mathrm{ml} \mathrm{LPS}$

Suppl. Movie 4: nIR response of $(\mathrm{GT})_{15}-\mathrm{SWCNT}$ s loaded in a neutrophil during addition of $100 \mathrm{nM}$ dopamine (no cell activation, cell \& chromatin geometry shown in fig S6a, grey dot marks timepoint of addition)

Suppl. Movie 5: nIR response of $(\mathrm{GT})_{15}-\mathrm{SWCNT}$ loaded in a neutrophil during addition of $100 \mathrm{nM}$ dopamine (after rupture (100 nM PMA used), cell \& chromatin geometry shown in fig S6a, grey dot marks timepoint of addition)

Suppl. Movie 6: nIR response of hemin coated aptamer-SWCNTs loaded in an unactivated, intact neutrophil during addition of $100 \mu \mathrm{M} \mathrm{H}_{2} \mathrm{O}_{2}$ (cell \& chromatin geometry shown in fig S6a, grey dot marks timepoint of addition)

Suppl. Movie 7: nIR response of hemin coated aptamer-SWCNT loaded in a ruptured neutrophil during addition of $100 \mu \mathrm{M} \mathrm{H}_{2} \mathrm{O}_{2}$ (cell \& chromatin geometry shown in fig S6a, grey dot marks timepoint of addition)

Suppl. Movie 8: Activation of patterned SWCNTs with the aid of $100 \mathrm{nM}$ dopamine addendum - grey dot marks timepoint of addition 
a

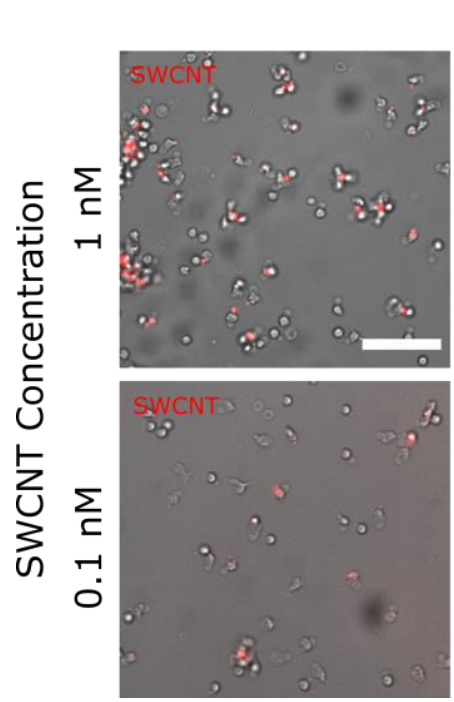

b
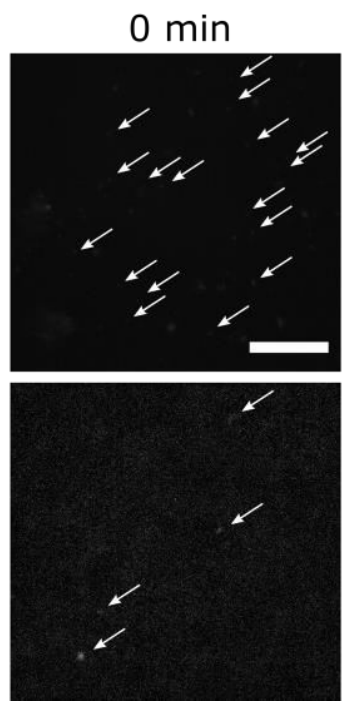

Incubation time

\section{$10 \mathrm{~min}$}
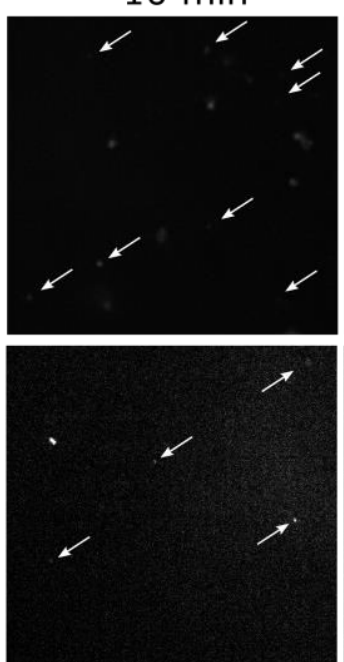

$30 \min$

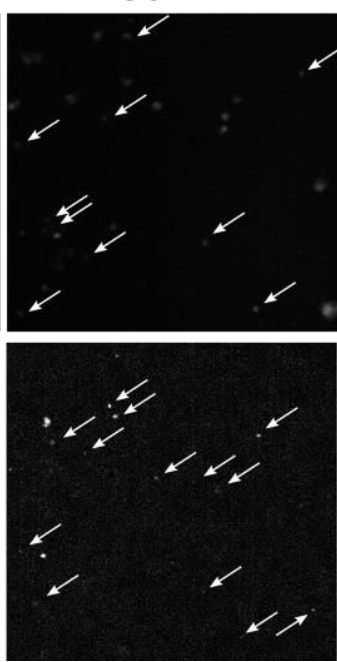

Fig. S1: Exemplary images of SWCNT uptake experiments. a Multichannel images of neutrophilic granulocytes after $(\mathrm{GT})_{15^{-}}$ SWCNT uptake. An incubation concentration of $1 \mathrm{nM}$ SWCNT for 30 minutes (top) led to enhanced sensor colocalization (red) in single cells but also cell agglomeration which prevented further migration of affected neutrophils. Lowering the SWCNT concentration to $0.1 \mathrm{nM}$ (bottom) reduced this process at the expense of sensor intensity within the cells. Normally, sensors could be found at the cell rear during migration while in cell agglomerations, SWCNT signals were located in the center of the cell bulk. Scale bar $=100 \mu \mathrm{m}$ b Near-infrared images of uptaken $(\mathrm{GT})_{15}$ SWCNTs for different amounts of incubation time. SWCNT intensities in cells increased with prolonged contact periods and starting concentration of the sensors. Scale bar $=100 \mu \mathrm{m}$, the contrast of bottom images is increased to show sensor positions. 


\section{PMA}
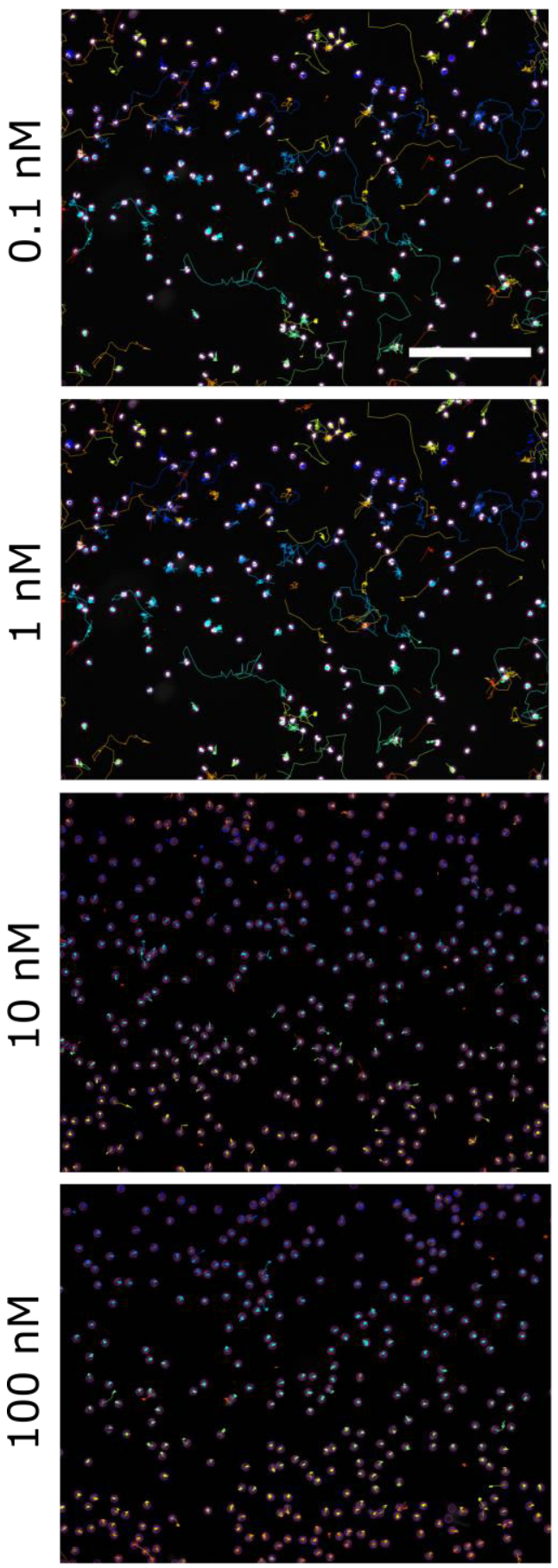

\section{Control}

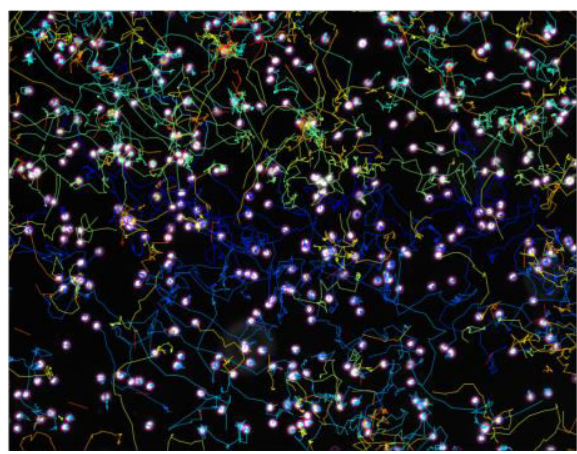

LPS
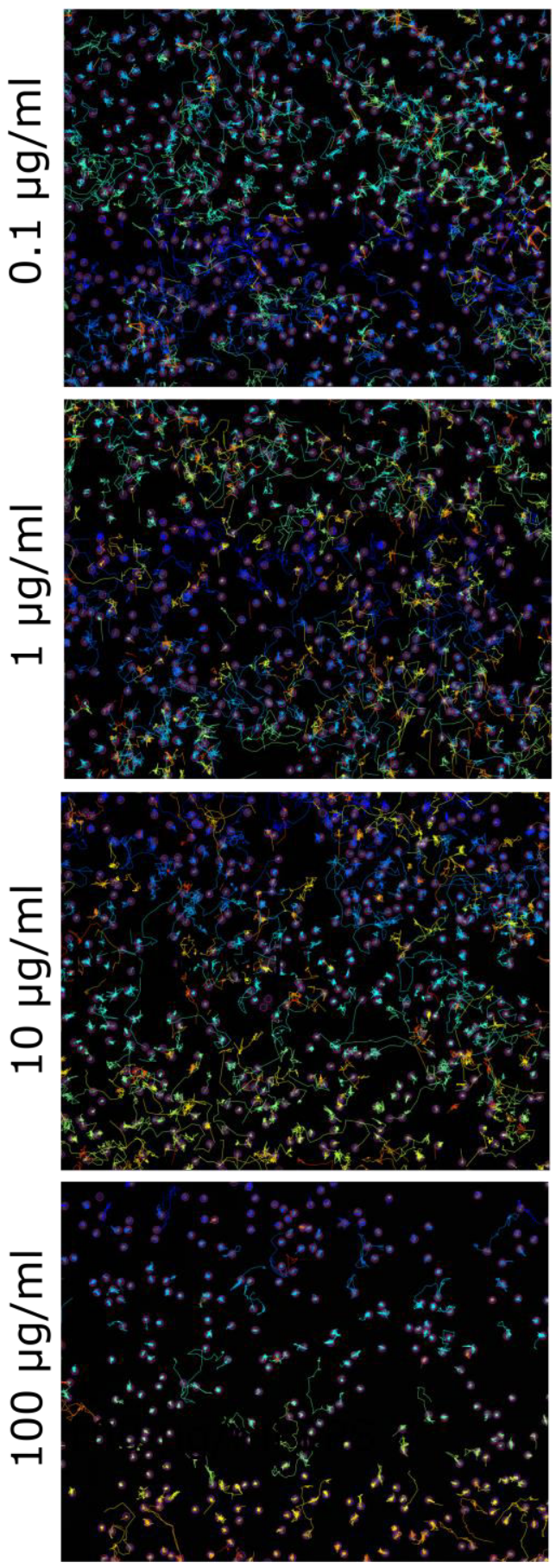

Fig. S2: Migration traces of preactivated neutrophils for different concentrations of NET-formation transducers. Using PMA $(0.1-100 \mathrm{nM})$ and LPS $(0.1$ $-100 \mu \mathrm{g} / \mathrm{ml})$ as activators to stimulate different NETformation pathways, the migration behavior of activated neutrophils were investigated. Lower amount of PMA led to similar speed and stopping times of migrating cells compared to control samples. On the contrary, $10-100 \mathrm{nM}$ PMA forced nearly all cells to instantly stop. Lower amounts of LPS did not change the movement pattern of the cells, however increasing concentrations lowered the cells speed and locomotion duration. Hoechst stain were used for nuclei staining, migration traces were generated by TrackMate ImageJ plugin, scale bar $=100 \mu \mathrm{m}-$ traces show migration pattern after 160 minutes of consistend recording. 


\section{PMA}
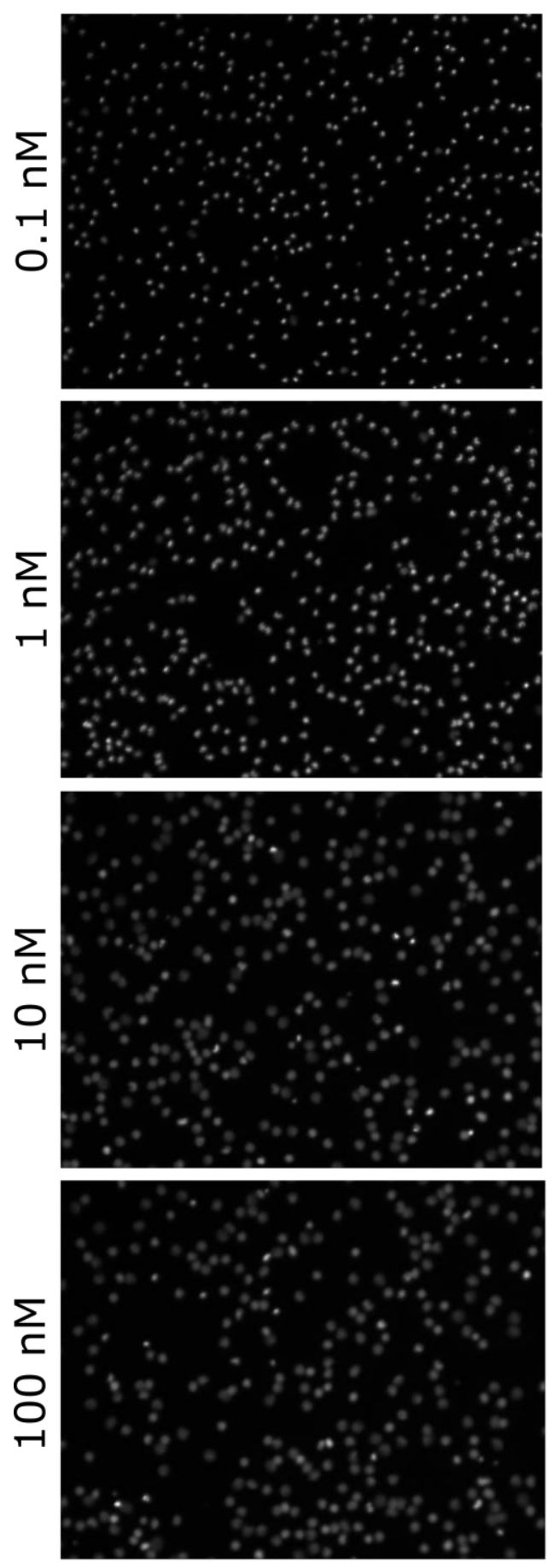

\section{Control}

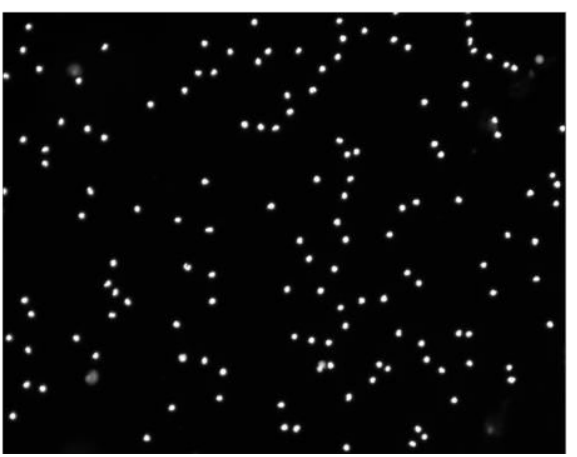

\section{LPS}
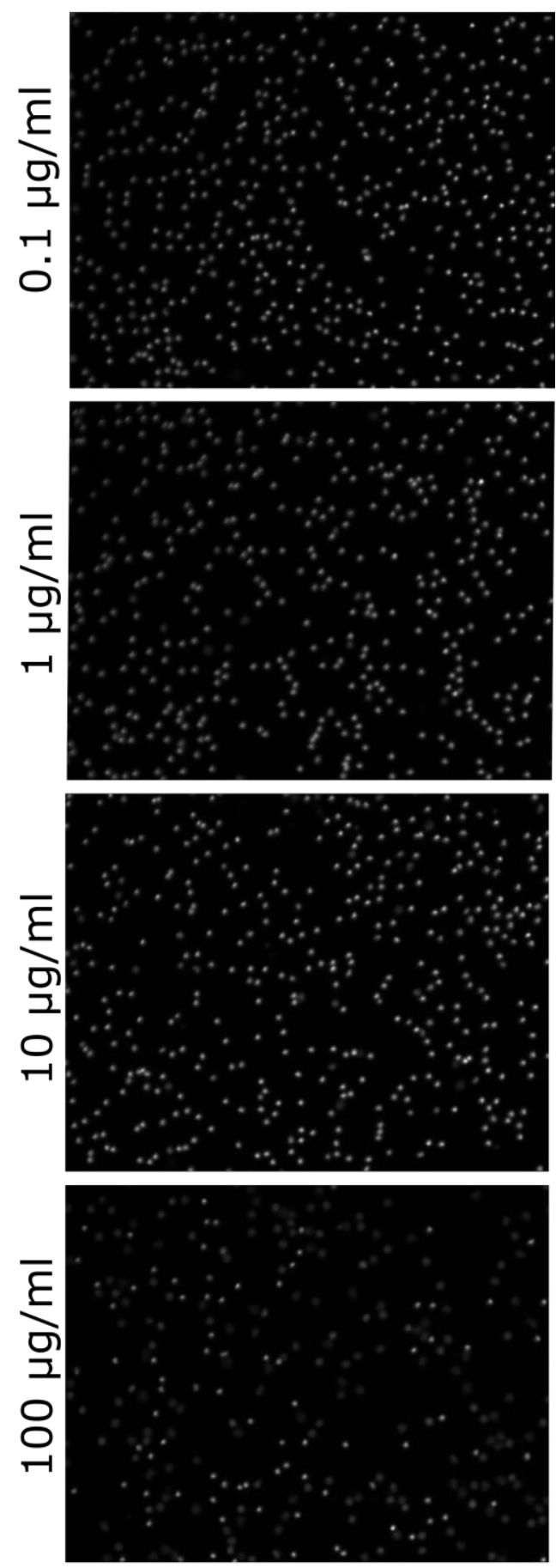

Fig. S3: Decondensation behavior of preactivated moving neutrophils after 160 minutes. Fluorescence pictures of activated neutrophil nuclei 160 minutes after activation. Both, lower amounts of LPS and PMA (i.e. $0.1-1 \mathrm{nM}$ PMA and $0.1-1 \mu \mathrm{g} / \mathrm{ml} \mathrm{LPS}$ ) did not show any significant decondensation compared to control samples. In contrast, 10 $100 \mathrm{nM}$ PMA resulted in a nearly complete decondensation of all cells while only 100 $\mu \mathrm{g} / \mathrm{ml}$ LPS showed similiar yet less effective decondensation numbers. Hoechst 33342 stains, scale bar $=100 \mu \mathrm{m}$. 


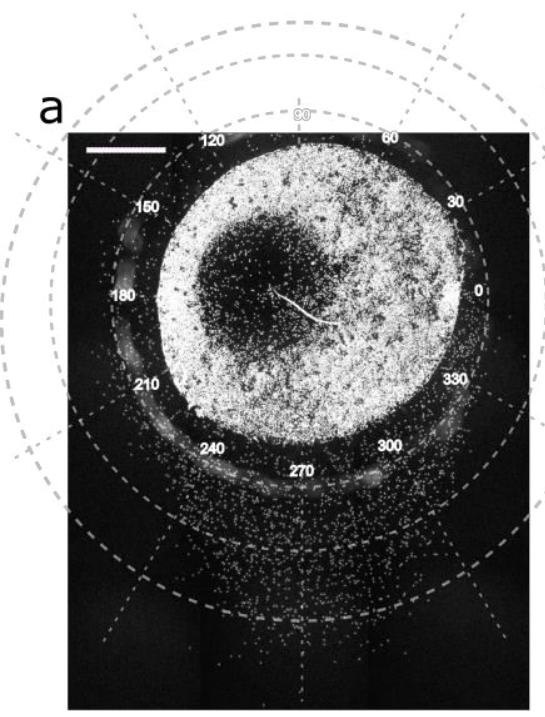

b PMA

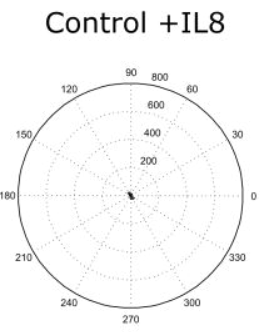

Control -IL8
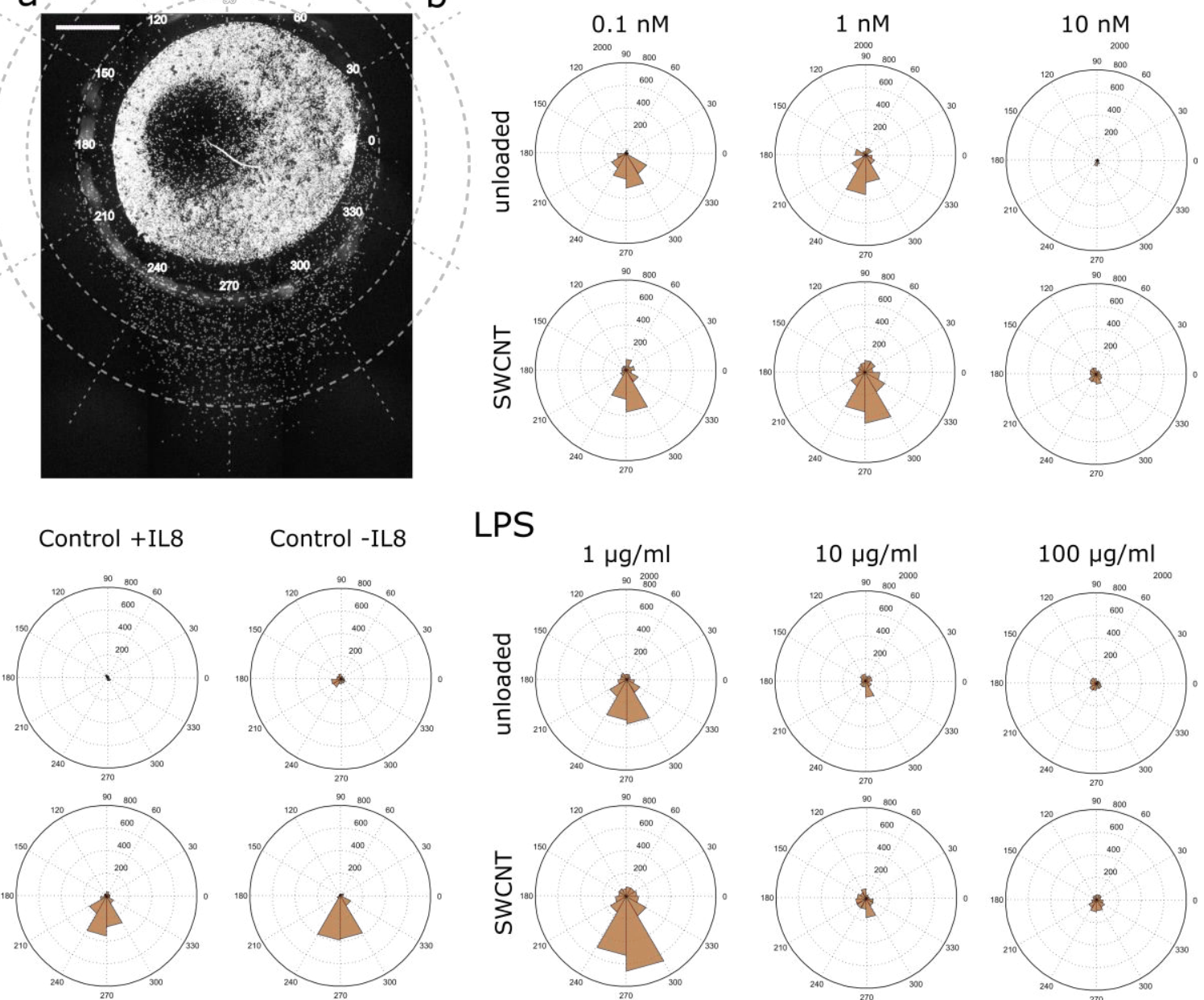

\section{LPS}
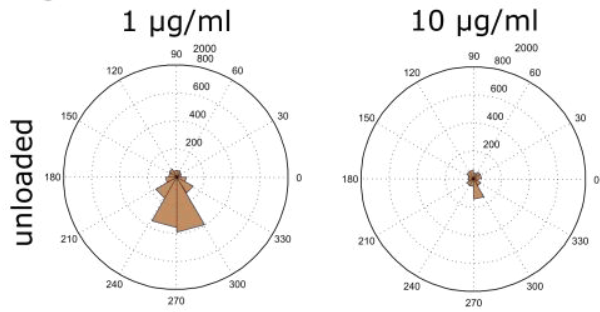

$100 \mu \mathrm{g} / \mathrm{ml}$
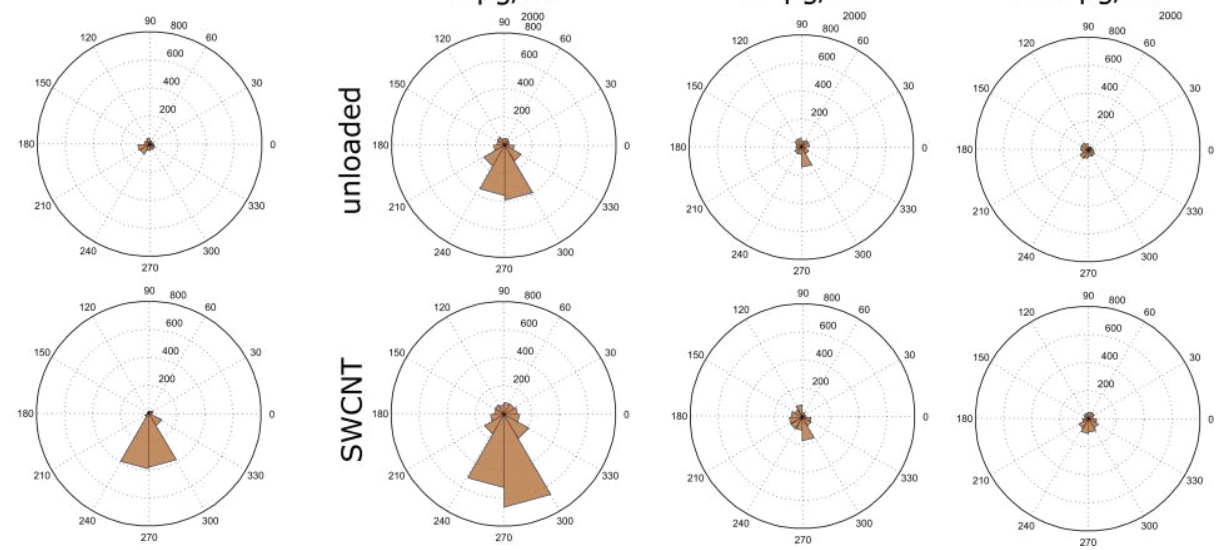

Fig. S4: Migration behavior of (GT) $)_{15}$-SWNT loaded and unloaded neutrophils in a migration-under-agarose experiment using different amounts of NETosis activators and $0.5 \%$ FCS inherited agarose gels. a Exemplary image of a typical under agarose experiment plus description of the used coordinate system. Cells were loaded in one well (diameter around $\mathrm{d}=3 \mathrm{~mm}$ ) and activated shortly before the chemoattractant $(\mathrm{IL}-8,0.1 \mu \mathrm{M})$ was poured in a second well $(2.2 \mathrm{~mm}$ away from the cells) which generated a consistent gradient within the gel. Neutrophils were allowed to move freely for 3 hours and were imaged using Hoechst 33342 stain afterwards. Pictures were arranged so that the $270^{\circ}$ faces towards the IL- 8 well, scale bar $=400 \mu \mathrm{m}$. b Analysis of the migratory distance of (GT) ${ }_{15}$-loaded neutrophils and untreated ones after 3 hours within an under-agarose sample. Cells with SWCNTs showed enhanced movement compared to those without any pretreatment. Increasing the concentrations of LPS or PMA resulted in reduced locomotion in both cases. $\mathrm{N}=3$, Data shows mean values in $\mu \mathrm{m}$. 
a
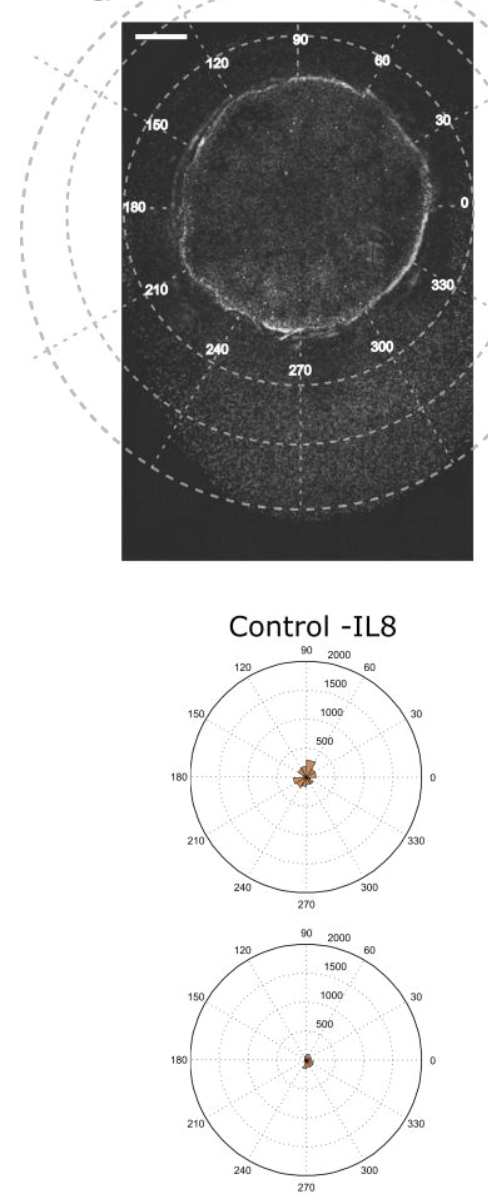

\section{PMA}

b
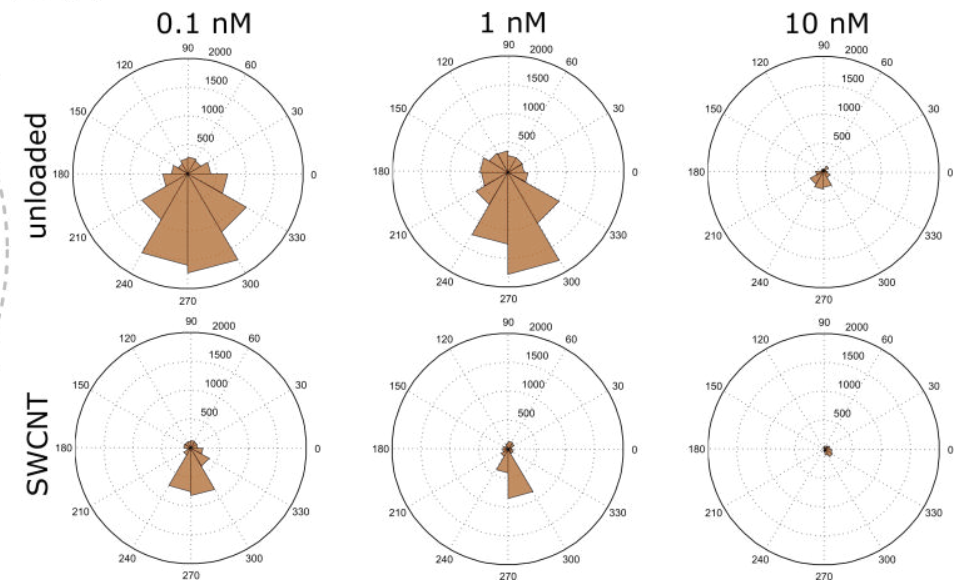

LPS
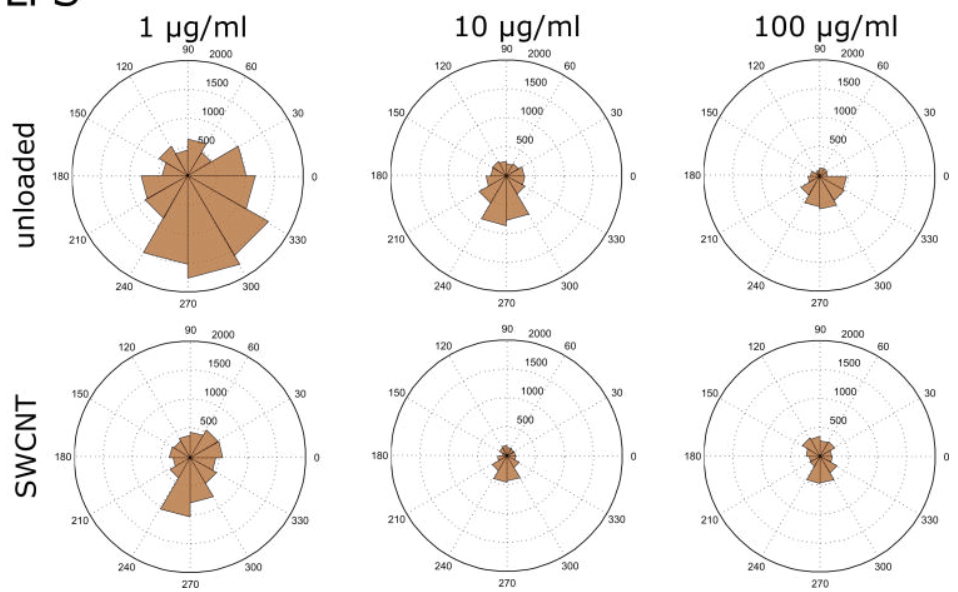

Fig. S5: Migration behavior of (GT) $)_{15}$-loaded and unloaded neutrophils in a migration-under-agarose experiment using different amounts of NETosis activators and $20 \%$ FCS inherited agarose gels. a Exemplary image of a typical under agarose experiment plus description of the used coordinate system. Cells were loaded in one well (diameter around $\mathrm{d}=3 \mathrm{~mm}$ ) and activated shortly before the chemoattractant (IL-8, $0.1 \mu \mathrm{M})$ was poured in a second well $(2.2 \mathrm{~mm}$ away from the cells) which generated a consistent gradient within the gel. Neutrophils were allowed to move freely for 3 hours and were imaged using Hoechst 33342 stain afterwards. Pictures were arranged so that the $270^{\circ}$ faces towards the IL- 8 well, scale bar $=500 \mu \mathrm{m}$. b Analysis of the migratory distance of $(\mathrm{GT})_{15}-\mathrm{loaded}$ neutrophils and untreated ones after 3 hours within an under agarose sample. Cells without SWCNTs showed enhanced movement compared to sensor-containing neutrophils and even reached the other well sometimes. Increasing the concentrations of LPS or PMA resulted in reduced locomotion in both cases. $\mathrm{N}=2$, Data shows the mean value of both experiments in $\mu \mathrm{m}$. 
a
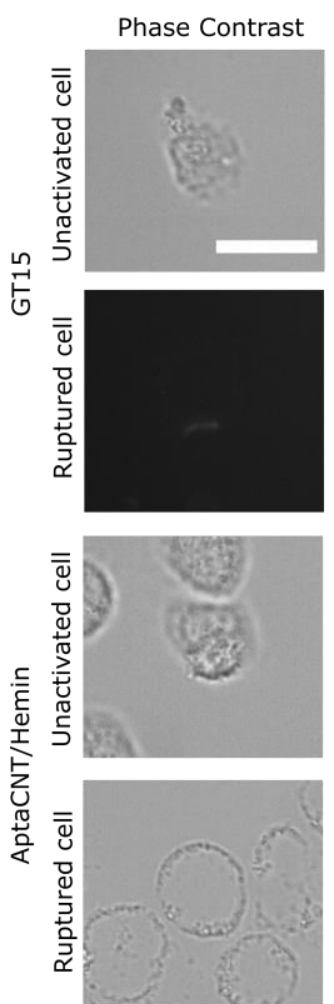

Chromatin
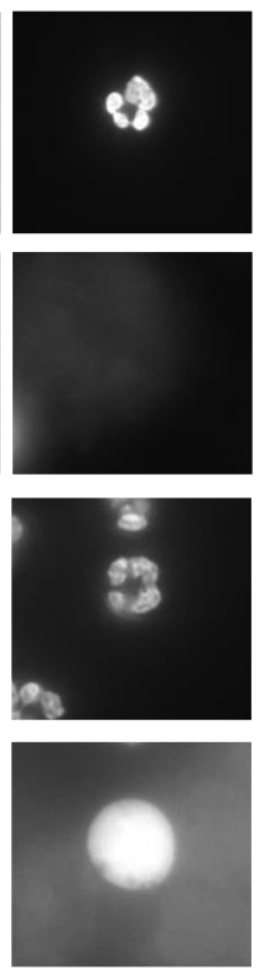

b
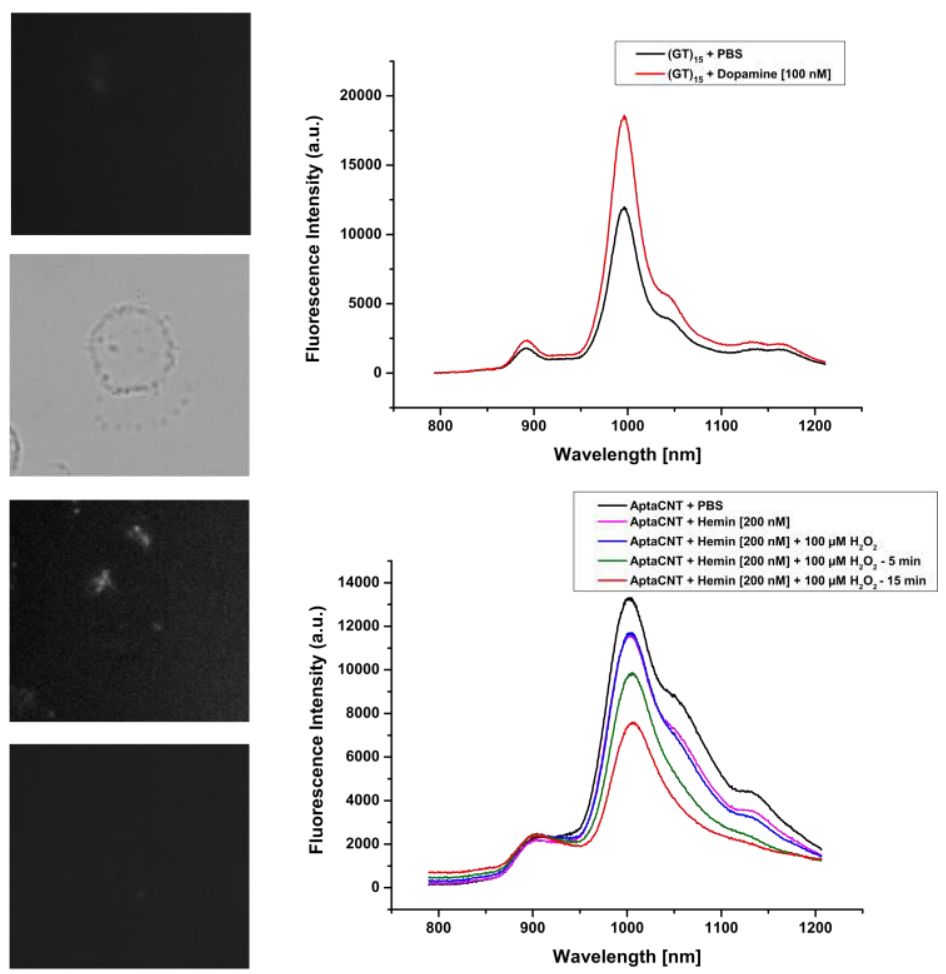

SWCNT
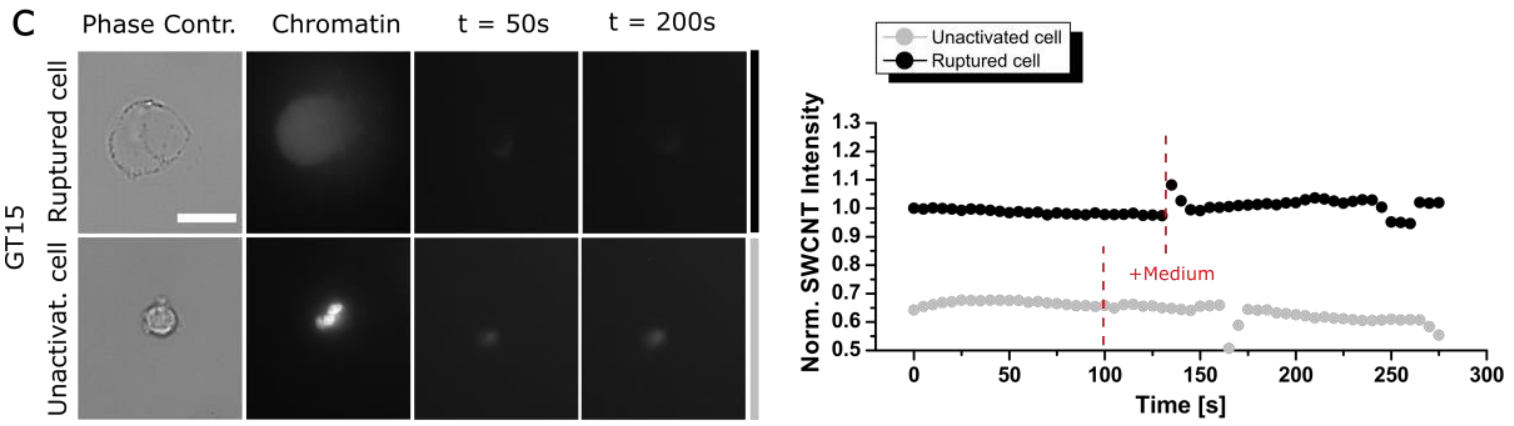

d
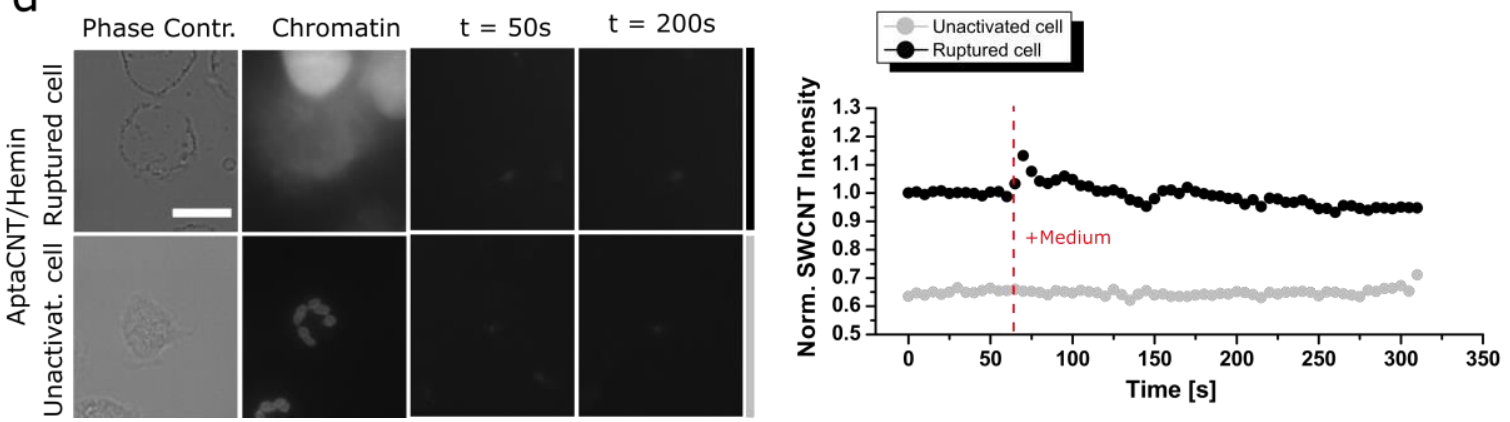

Fig. S6: Optical response of (GT) $)_{1-}^{-}$and hemin/aptamer functionalized SWCNTs. a Exemplary cell and sensor pictures used to depict the SWCNT responses shown in Fig. 5a (respective SWCNT movies can be seen in Suppl. movie 5-8). The contrast of SWCNT pictures were enhanced to show sensor geometry and position. Scale bar $=10 \mu \mathrm{m}$. b Excitation spectrum of (GT) ${ }_{15}$ (top) and aptamer/hemin functionalized SWCNT (AptaCNT/Hemin, bottom) dissolved in PBS. Adding $100 \mathrm{nM}$ dopamine to a (GT) 15 sample resulted in a $40 \%$ increase of its overall intensity whereas $100 \mu \mathrm{M}$ of $\mathrm{H}_{2} \mathrm{O}_{2}$ resulted in a steady decrease using aptamer/heminSWCNTs. c Intensity analysis of loaded (GT) ${ }_{15}$-SWCNTs while adding RPMI-Medium as a control. Both, intact, unactivated cells (bottom) as well as already ruptured cells (top) were used and showed no overall change of intensity before or after addition of $500 \mu 1$ RPMI-medium. d Equal control samples using aptamer/hemin-SWCNTs plus RPMI-medium. Likewise, no measurable changes could be found. Scale bar $=10 \mu \mathrm{m}$, chromatin stained by $100 \mathrm{nM}$ Hoechst 33342 . 


\section{Supplemental methods}

nIR fluorescence spectroscopy

nIR fluorescence spectra were recorded with a Shamrock 193i spectrometer (Andor Technology Ltd., Belfast, Northern Ireland) connected to an IX53 microscope (Olympus, Tokyo, Japan). Excitation was performed with a $561 \mathrm{~nm}$ Cobolt Jive ${ }^{\mathrm{TM}}$ laser (Cobolt AB, Solna, Sweden) for (GT) ${ }_{15}$-SWCNT samples or with a $785 \mathrm{~nm}$ laser (OBIS LX, Coherent) in case of Aptamer functionalized SWCNTs.

To test (GT) ${ }_{15}$-SWCNTs responses to dopamine, $180 \mu 1$ of a $0.1 \mathrm{nM}$ ssDNA/SWCNT solution in PBS was placed in a 96-well plate, measured and $20 \mu 1$ of $1 \mu \mathrm{M}$ dopamine was added subsequently to yield a final concentration of $100 \mu \mathrm{M}$. The fluorescence response of the hemin coated aptamer-SWCNT was studied likewise by mixing $20 \mu 1$ of $1 \mathrm{mM}$ $\mathrm{H}_{2} \mathrm{O}_{2}$ solution in PBS to $180 \mu \mathrm{l}$ of $2 \mathrm{nM}$ Aptamer-SWCNT with $200 \mathrm{nM}$ hemin in it. 


\section{Publication Synopsis \& Contributions}




\subsection{Manuscript 1}

Chromatin Swelling Drives Neutrophil Extracellular Trap Release, Neubert E`., Meyer D. ${ }^{\ddagger}$, Rocca F., Günay G., Kwaczala-Tessmann, A., Grandke J., Senger-Sander S., Geisler C., Egner A., Schön M. P., Erpenbeck L., Kruss S., Nat. Comm. 9(1), 3767, DOI: 10.10384/s41467-018-06263-5 (2018)

This project represents the first biophysical characterization of NETosis and, simultaneously, the first time-resolved investigation of its mechanical and morphological consequences on associated immune cells (neutrophils). With the aid of fluorescence live cell microscopy, first, a characteristic time pattern was determined and enabled the division of NET-formation into three consecutive phases: a first phase, defined by the neutrophils firm adhesion and the existence of its intact, lobular shaped nuclei (P1), a second phase predominantly identified by the rupture of the nuclear membrane and a subsequent chromatin swelling (P2) and a third phase represented by the final breakdown of the cellular membrane and the release of NETs (P3). Interestingly, following metabolic tests, including measures of the cellular ATP levels, the utilization of several inhibitors and temperature variations showed significant distinctions between P1 and P2 and indicated an overall phase shift of the process. Here, as chemical modification affected NETosis only within the first phase, main processes in P1 were presumed to be of active, enzymatic-driven nature whereas prime movers in P2 were put in context with entropic swelling forces generated by the freed chromatin. Further tests underlined this statement by visualizing the correlation between the swelling dynamics and the overall geometry of the corresponding cell. Larger cells, for example, were found to rupture later and both, the location of the former nucleus as well as the shape of the neutrophilic membrane could be used to predict the appearance of the subsequent cell rupture point. Likewise, the generated "pushing" forces of the swollen chromatin could be identified as a potential trigger for the final cell burst since pressure values generated by life-cell AFM measurements and a theoretical model system illustrated sufficient capabilities to destroy a lipid bilayer. These insights then, in combination with the additionally found degradation of the neutrophils cytoskeletal stability, provided a potential, biophysical description of the NETotic process: NETosis is a multi-phasic mechanism. Agitated by initial chemical stimuli, active enzyme interactions weaken the intercellular architecture first and enable the expansion of the destabilized chromatin network. After this point, the point of no return, the outcome of NETosis cannot be altered anymore and lead to a rupture of the cellular membrane due to a combination of growing intracellular pressure and fading cytoskeletal stability.

These authors contributed equally

\section{Contribution}

General: Experimental design was planned together with all authors; major manuscript parts with Sebastian Kruss, Luise Erpenbeck and Elsa Neubert (E.N.); design and execution of the associated theoretical model; design and writing of all used Matlab codes.

Data generation \& analysis: Figures: Fig 2b, Fig. 4e, Fig. 4a/d (raw data by E.N.), Fig. 5d/e, Fig. 6a-e (raw data by E.N.), Fig.7 (together with E.N.); generated analysis code for data sets used in Fig. 1b-e, 3c, 4a/b/d; Supplementary figures:Supp. Fig. 4c/d (together with E.N.); Supp. Fig. 8d; Supp. Fig. 9a (raw data by E.N.); Supp. Fig. 10, Supp. Fig 11 (raw data by E.N.), Supp. Fig 13 (together with E.N.), supplied analysis codes for Supp. Fig. 1, 3, 8a; Supplementary movies: Suppl. Movie 9-11; Suppl. Movie 16 


\subsection{Manuscript 2}

Kinetic Requirements for Spatiotemporal Chemical Imaging with Fluorescent Nanosensors, Meyer D., Hagemann A., Kruss S., ACS Nano 11(4), pp 4017-4027, DOI: 10.1021/acsnano.7b00569 (2017)

As a second project, this study created a theoretical framework capable of emulating the spatiotemporal performance of nanosensor arrays for chemical imaging purposes. Primarily built to estimate the dynamical sensor properties (association/disassociation constants $\mathrm{k}_{\mathrm{on}} / \mathrm{k}_{\mathrm{off}}$ ) required by real-life nanosensors to sense and translate evanescent biological processes, a computational model was developed that mimicked the properties of chemical imaging experiments (analyte and sensor dynamics, optical setup limitations, etc.) and ascertained the best sensor settings for a given biological problem. For this purpose, the rapid release of dopamine neurotransmitters by chromaffin cells were chosen as a viable model system. Dynamic distributions of such molecules were simulated using two- and three-dimensional diffusion algorithms and consecutively placed on top of a theoretical sensor surface to generate a time-dependent analyte environment. The apparent analytesensor responses were then generated by a two-step procedure: firstly, binding and unbinding processes of single analytes were emulated by Gillespie-based Monte Carlo simulations and transferred into normalized intensity signals for each corresponding sensor (see also chapter 2.3). Secondly, such signals were redistributed to the given sensor location and affected by Airy disks and averaging procedures to mime optical setup restrictions such as diffraction limits or camera exposure times. Thus, a routine could be generated that is able to simulate the sensor response for any desired analyte distribution and transform it into an optical signal usually seen in real-life experiments. The detection of the best sensor properties was then accomplished by a combination of screening and image evaluation algorithms. Changing the binding dynamics of the sensor binding sites consecutively and evaluating the so generated sensor response images for the best spatial or temporal resolution conditions, a general heatmap was generated that

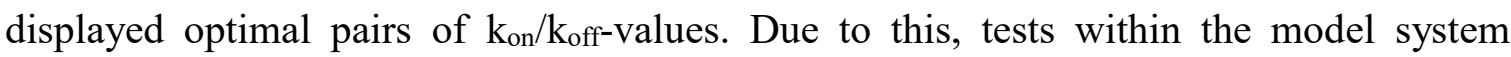
showed that optimal detection of dopamine release events requires rather high sensor dissociation properties $\left(\mathrm{K}_{\mathrm{d}}=100 \mu \mathrm{M}\right)$, primarily as the response to such fast, diffusive events necessitate a similar swift capability of sensors to switch (binding) states. Thus, as a conclusion, this framework may serve as a guideline for prospective sensor designs and underlines the necessity of fast responding interaction units, especially for biological applications.

\section{Contribution}

General: Manuscript design was planned together with all authors; the manuscript was written with Sebastian Kruss; arrangement of all figures; development of main code; usage of all codes and simulation platforms together with Annika Hagemann (A.H.); supervision of A.H.

Data generation \& analysis: Figures: Head figure; Fig. 1; Fig. 2a/b (raw data by A.H.); Fig. 3-4; Fig. 5a/b/e/f; Fig. 5c/d (raw data by A.H.); Fig. 6a/b; Fig. 7 (Data generation together with A.H.) Supplementary figures: Supp. Fig. 2; Supp. Fig. 4-5 (produced together with A.H.); Supplementary movies: Suppl. Movie1-5 


\subsection{Manuscript 3}

Programmed Transport and Release of Nanoscale Cargo, Meyer D., Telele S., Zelena A., Neubert E., Nissler R., Mann F., Grandke J., Erpenbeck L., Köster S., Kruss S., in preparation

Combining the knowledge of the previous two studies, the third work of this thesis aimed to utilize the unique multi-phase mechanism of NETosis and transform activated neutrophils into programmable carry-and-release systems for nanotube-based nanomaterials. For this, first, fluorescence-based live-cell tracking experiments confirmed the general capability of activated neutrophils to migrate and open up/exert NETosis consecutively. Using different types and concentrations of inducer molecules (PMA and LPS), specific conditions could be found in which stimulated cells demonstrated both, prolonged migration times as well as subsequent high release/rupture rates. "Under agarose" migration assays further substantiated this finding and validated the response of activated neutrophils to external influences (the chemokine IL-8). Here, while the behavior of SWCNT-laden cells did not differ significantly from associated control groups, even neutrophils effected by higher transducer concentrations could follow implemented IL-8 gradients, albeit the fact that they covered less distance in the end.

In addition to that, the functionality and uptake dynamics of the nanometer-sized cargo was addressed. By incubating neutrophils with the right amount of DNA-conjugated SWCNTs, the absorption of the functionalized material could be detected after tens of minutes and led to a characteristic deposition at the neutrophils rear. Astonishingly, neutrophils were then apparently able to seal off their inherent load as subsequent analyte (dopamine) addition was not followed by a visible change of SWCNT intensity. Nanosensors found in ruptured cells, in contrast, exhibited a typical sensor response and thus remained functional throughout the NETotic process. A final cell patterning experiment then concluded this project and visualized the strength of neutrophil-based carrier systems once more: due to the multi-phase nature of NETosis and the unique properties neutrophilic granulocytes provide, these special kinds of transporter units hold great potential for in vitro and possible in vivo applications. They are able to take up functional nanomaterials, convey them to specific sites of interest and can be programmed to release their cargo (NETosis) in a time-dependent manner - a combination unmatched by any conventional delivery system.

\section{Contribution}

General: Experimental design was planned together with all authors; the manuscript was written with Sebastian Kruss; production of all figures; development and usage of all Matlab codes; supervision of Saba Telele (S.T.); utilized cells were isolated by Elsa Neubert, utilized SWCNTs were produced by Robert Nissler \& Florian Mann

Data generation \& analysis: Figures: Fig. 1-3; Fig. 4a; Fig 4b (raw data by S.T.); Fig. 4c (captured with S.T.); Fig. 5 a/b; Fig. 5c (pattern preparation with Anna Zelena) Supplementary figures: Supp. Fig. 1-3; Supp. Fig. 4-5 (raw data with S.T.); Supp. Fig. 6a/c/d Supplementary movies: Suppl. Movie1-2; Suppl. Movie 3-4 (sample preparation by S.T.), Suppl. Movie 5-9 


\subsection{Cooperative publications}

Further cooperations led to following studies:

1. Tuning Selectivity of Fluorescent Carbon Nanotube-Based Neurotransmitter Sensors, Mann F.A., Herrmann N., Meyer D., Kruss S., Sensors 17(7), 1521, $10.3390 / \mathrm{s} 17071521(2017)$

2. Carbon Nanotubes Encapsulated in Coiled-Coil Peptide Barrels, Mann F., Horlebein J., Meyer N., Meyer D., Thomas F., Kruss S., Chemistry - A European Journal 24(47), 12241-12245; 10.1038/s41467-018-06263-5 (2018)

3. Quantification of the Number of Adsorbed DNA Molecules on Single-Walled Carbon Nanotubes, Nissler R., Mann F.A., Chaturvedi P., Horlebein J., Meyer D., Vukovic L., Kruss S., JPC 123(8), 4837-4847; 10.1021/acs.jpcc.8b11058 (2019)

\section{Contributions}

Building and adjustment of applied optical systems, initial supervision of AFM experiments, text writing and correction together with all authors

4. Effect of Adhesion and Substrate Elasticity on Neutrophil Extracellular Trap Formation, Erpenbeck L., Gruhn A. L., Kudryasheva G., Guenay G., Meyer D., Neubert E., Grandke J., Schoen M. P., Rehfeldt F., Kruss S., preprint at BioRxiv, doi: https://doi.org/10.1101/508366

\section{Contributions}

Polyacrylamide sample preparation together with Galina Kudryasheva, text writing and correction together with all authors 


\section{Discussion}

\subsection{NETosis - A Change of Paradigm}

In the last decade, the advances in NET-research have been truly astounding. A vast number of transducer molecules have been revealed and were, to some extent, related to diverse molecular pathways and pathological conditions (see also chapter 2.1.4). Both, mouse models as well as in vitro studies have brought profound insights into underlying chemical cues and associated diseases and aided (and certainly will further aid) the design of new therapeutic approaches. Yet, time-resolved investigations of NETosis have never been conducted in a detailed fashion, let alone described biophysically, which establishes the findings of chapter 3.1 as an entirely new starting point for prospective NET-studies. As this work showed, NETosis must be considered as more than just a mechanism of biochemical signaling. Rather it is a complex process that is comprised of distinct metabolic phases, each of them defined by a characteristic timeline and driving force, and thus provides far more facets and opportunities than initially anticipated. For this reason, these findings represent a crucial change of paradigm for the field of NETosis. They are likely to lead to new approaches of modulating the outcome of this novel, immunological process and will therefore be comprehensively discussed within the following sections.

\subsubsection{NETosis - Open the Chaos}

Metaphorically speaking, the neutrophilic nucleus could be compared to greek mythologies Pandora's box: Once it is opened, chaos is released and will dominate its surrounding - in this case, the cell. Here, what might appear to be an odd comparison at first, translates into an ideal simile for NETosis if the word chaos is replaced by its more physicochemically adequate term entropy. As extensively discussed in chapter 3.1, the finding of entropic forces as a dominant role in NET-formation is a focal outcome of this study. It allowed the development of the aforementioned phase model, explained the final cell rupture and enforces the reassessment of previously stated viewpoints.

One of these viewpoints refers, for example, to the general functions of the neutrophilic nuclear body. Usually protecting the cell's control center and securing the structural requirements for its complex, transcriptional mechanisms, in case of NETosis, it should now also been seen in a new function: It acts as a mechanical barrier. In this role, it holds the central position within the complex biological process of NET release and dictates its starting time, the point of no return, by tight balancing forces between its condensed (yet pushing) chromatin network, damping membrane deformations and its cytoskeletal con- 
nection points. As a consequence, one might, in accordance with chapter 3.1, re-envision NETosis from a new, mechanical point of view and discuss how this process is able to shift such balance of forces and achieve its final goal - the release of nuclear content.

Here, besides the known, direct modification of associated histones ${ }^{208}$, one option might be the removal of stabilizing, intracellular structures. Within chapter 3.1, we were able to strengthen the previous hypothesis of Metzler et al. that a degradation of the cytoskeleton is a requisite for NET-formation. ${ }^{209}$ As we were able to demonstrate, an initiation of NETosis entailed massive morphological changes for the cell starting from the diminishment of main cytoskeletal components and leading to the softening and even complete rounding events of the cellular structure (chapter 3.1, figure 6b-e). Simultaneously, fluorescence stainings on fixed samples showed nearly complete degradation of F-actin and microtubules within P1 (chapter 3.1, figure 5a) accompanied by an impressive loss of tether tensions and migratory cell functions (chapter 3.1, figure 5d-e; chapter 3.3, figure 3). It is therefore increasingly apparent that the excision of the cytoskeleton (and with it one of the most cell stabilizing factors) is vital to exceed the point of no return, not least because the addition of Jasplakinolide, as a potent actin-depolymerization antagonist, likewise inhibited NETosis (chapter 3.1, figure 5c). Additionally, previous studies further support this idea: the above-mentioned study of Metzler et al., for instance, directly correlated actin degradation with the release of $\mathrm{NE}$ and underlined their statement with functional inhibitor assays. ${ }^{209}$ Furthermore, an earlier report of Papayannopoulos et al. showed swelling behavior of isolated neutrophilic nuclei in in vitro experiments, indicating the fragile nature of the nuclear envelope and the necessity of an active, functional environment to secure its lobular shape. ${ }^{106}$ Thus, these findings, in combination with our results, highlight the importance of the mechanical balance of forces the cytoskeleton exerts. Concurrently, they emphasize the requirements of cellular destabilization in order to form the NETotic effects of chromatin swelling, along with the nuclear and cellular membranes' rupture.

Secondly, when taking the aforementioned findings into consideration, also the mechanical stability of the nuclear envelope itself must be taken into account as a crucial factor for NET-formation - especially as its lobular shape in combination with its unique envelope composition might render it more prone to destructive effects, such as intracellular shear or swelling forces. Indeed, it is already a well-established fact that neutrophilic nuclei are comprised of fewer lamina proteins, including small amounts of lamin $\mathrm{A} / \mathrm{C}^{210}$ and weak linker of nucleoskeleton and cytoskeleton (LINC) complexes ${ }^{211}$, which makes it tempting to speculate that their fragile nature facilitates NETotic effects and promotes a nuclear breakdown. Reports of nuclear rupture events during small pore transmigration underline this hypothesis. ${ }^{212}$ Correspondingly, by observing laminB1 labeled cells, we also noticed most often an opening of the lamina at positions of maximal instability (larg- 
est distance from appearing negative curvatures within the nuclear body, see for example chapter 3.1, figure 2c, supp. fig. 5). Considering that these events were synchronized with the start of $\mathrm{P} 2$, this appears to be a sufficient indication for the mechanical fragility of the neutrophilic nucleus and its impact on NETosis. Vice versa, this would also explain the recently found significance of specific pore-forming proteins, such as GasderminD. While so far GasderminD is only speculated to influence granular pore formation, and thus enhance release and translocation of intracellular NE, it could likewise undermine the structure of the cellular/nuclear envelope and thereby mechanically support the final NET-release as well. ${ }^{213}$ As a consequence, it would be interesting to explore if alterations of the nuclear geometry or composition might directly influence NET-formation. Neutrophils of mice or HL-60 cell models have, as an example, already proven to possess more lamins $^{214}$, be less fragile within methanol fixation protocols ${ }^{215}$ and exert less NETosis ${ }^{216}$. Yet, they have not been actually correlated in terms of their NETotic-mechanical potential thus far. In this regard, follow-up research covering these cell-types and live-cell imaging experiments, as presented in chapter 3.1, figure 1b, could lead to intriguing new insights. It could, for example, elucidate if stronger nuclear envelopes indeed resist NETosis, whether they alter onsets of P2 or P3 or even facilitate alternative methods to inhibit swelling forces.

To that end, a final possibility to influence the force balance within NETotic neutrophils remains to be discussed: The manipulation of the nuclear content itself. Here, while being the initial inducer of chromatin decondensation, the structural consequences that histone degradation cause on the chromatin framework are so far relatively unknown; this is, unfortunately, due to technical inabilities to account for substructural information, as shown by our STED images (chapter 3.1, figure 4c). Nevertheless, the importance of the underlying chromatin composition is difficult to overlook: the more histones are modified by enzymes such as NE or PAD4, the less the associated DNA-polymer is restrained to its original geometry and should therefore release stronger entropic powers. Hence, one might speculate that, for instance, different stimuli are capable of modifying the nuclear content specifically and with that facilitate and define the dynamics of a given NETformation. Here, a first support for such a hypothesis can be likely seen in a recent study of de Bond et al.. ${ }^{217}$ Using PMA, LPS and calcium ionophore as activators to stimulate different pathways of NETosis and comparing the amount of subsequent released DNA, they were able to account for more "loosened" structures in case of LPS-activation while PMA-induced NETs seemed to stay rather mingled. This result, in combination with our rather short duration of P2 measured for LPS-stimulated cells (chapter 3.1, supp. fig. 3d), does not only further strengthen the importance of entropic forces on NET-dynamics but might also lead to new possibilities to characterize or even predict the efficiency of a given stimulus. 
Hence, one might recognize the significant advantages such a change in perspective can generate, especially in cases of such a process that is so purely biochemically contemplated thus far as NETosis. In such cases, it helps to visualize so far unrecognized factors and can set thought-provoking impulses towards new, valuable follow-up research. Therefore, the biophysical approach we presented in this study should also not only be viewed as a "new" concept to analyze NET-formation but can be likewise seen as a motivation to integrate further areas of research. On this occasion, particularly application- and modeloriented fields, such as theoretical physics or bioengineering could lead to new, interesting, if not even essential, findings for prospective NET-research. 3D-simulations of expanding, spatially limited (chromatin) networks could, for example, become a vital tool to understand the intracellular conversions neutrophils experience in P2. Additionally, the development of artificial, membrane-stabilizing proteins, such as reported by Veith et al., might represent another exciting aspect combining biotechnology and biophysical approaches. ${ }^{218}$ While such proteins could be used to further investigate the influence of the membrane's stability on NETosis, it could also bring forth new therapeutic strategies capable of preventing NET-release and thus NETosis-related diseases.

\subsubsection{NETosis - A Force Speculation}

In addition to the preceding, broad discussion in chapter 3.1, one might also reflect upon the magnitude of swelling forces covered in this study, particularly in order to discuss whether or how such dimensions are finally able to disrupt the cellular membrane. In doing so, the fact that NETosis, or even a neutrophil itself, was hitherto never related to intracellular force measurements delineates the results of this study as groundbreaking work while also enforcing a rather broad comparison to other cell types and experimental conditions. Nevertheless, and as already discussed in chapter 3.1, using live-cell AFM measurements (chapter 3.1, figure 4e) as well as Navier-Stokes equation-based pressure estimations (chapter 3.1, figure 4d) we were able to verify an intracellular pressure increase of around $100-150 \mathrm{~Pa}$ shortly before the cell's rupture and could attribute it to chromatin swelling due to the close temporal correlations to $\left[\mathrm{t}_{2} ; \mathrm{t}_{3}\right]$ (duration of completely filled cell body). With this, it should be roughly estimated as a doubling of the cells original pressure amount, since present examinations of other cell types, such as HeLa cells $^{219}$, Xenoplus blastomers ${ }^{220}$ or keratocytes ${ }^{221}$, demonstrated resting values in orders of $50-200 \mathrm{~Pa}$. Reports of systematic and well-assessed cell ruptures, in contrast, are unfortunately rare or cover rather cell compression or bending events instead of swelling. ${ }^{22}$ A study of Kelly et al., could eventually function as a first guideline, as they inflated Xenopus oocytes using high hypotonic media, which resulted in final intracellular pressure values of around $500-700 \mathrm{~Pa}^{223}$ Likewise, Koslov et al. lysed large vesicles osmotically and could account for rupture pressures of around $100-200 \mathrm{~Pa}$, indicating that our 
measured swelling forces are at least in the right order of magnitude to burst a cellular membrane. ${ }^{224}$ Interestingly, mitosis in HeLa cells, which was only recently found to hold surprising similarities to the NETotic process ${ }^{225}$, were reported to equally direct cell reconstruction by osmotic pressures ranging in similar scales $(80-260 \mathrm{~Pa}){ }^{219,226}$

At the same time, one might also take into account that activated neutrophils undergo a range of mechanical and geometrical alterations that might equally affect the generated swelling forces. The continuous degradation of cytoskeletal components discussed in the previous section might, for instance, further destabilize the neutrophil and enhance its susceptibility by introducing local inhomogeneities within its membrane tension. Therefore it is likely that even smaller stresses are sufficient to burst the cellular membrane. Here, similar effects might be expected for the demonstrated cell rounding (chapter 3.1, figure 1a) and membrane segregation events (chapter 3.1, supp. fig. 4a,b). While the former should apparently decrease the neutrophil's ductility by eliminating parts of its supporting excess-membrane, it is reasonable to assume that a change of the cell's geometry likewise entails vast, intracellular modifications and a destabilization of its basic structure. Intriguingly, this would also explain the reports of $\mathrm{Yu}$ et al. who encountered increased cell lysis after cell activation in various shear stress environments. ${ }^{227}$ Albeit the fact that neutrophils are known for their sensitivity to external mechanical factors, such findings might only indicate that NETotic cells hold an enhanced vulnerability to disruptive strains and thus favor the effect of any physical, cell-damaging source. All the more it becomes persuasive that the swelling forces measured in chapter 3.1, figure 4d might exploit this cellular "weakness" and introduce the last phase of NETosis by a final push on the pre-destabilized, neutrophilic membrane.

Beyond that, it is also of central importance to include the results of chapter 3.1, figure 6 (geometrical analysis of the breaking point) into the discussion, predominantly as they serve as a link between our experimentally determined rupture pressures and the dynamical consequences of their associated chromatin networks. Within this passage, we were able to systematically relate the momentum of specific cell compartments to the location of the associated rupture point. More specifically, we demonstrated that the final position of the cellular disruption was most often found either at sites of maximal chromatin density (and thus of maximal swelling forces, chapter 3.1, figure 6a) or places of fast membrane retraction (chapter 3.1, figure 6b-d) which likewise resulted in local chromatin decondensation. With this, these findings provide one fundamental and new insight: they unmistakably correlate the distribution of the expanding, nuclear content with the onset of the respective cell opening - or, in other words, they prove the direct impact of the chromatin decondensation on the final breakage of the cell.

As a result, one might rephrase the initial question in the following way: combining the perceptions of the results mentioned above, it seems undeniable that the forces generated 
by the swelling chromatin network are sufficient to burst the neutrophilic envelope. While the measured magnitudes, supported by cell-destabilizing effects, already emphasize their destructive potential during NETosis, the correlations described in the last paragraph are able to prove their impact independent from any specific force speculation. Besides, the results of chapter 3.1, figure 6a also deduce a second perception worth mentioning: the necessity of multidimensional approaches to study NETosis. As these findings showed, the cell opening is usually found in locations of enhanced chromatin density, indicating local peaks of (swelling) pressures and an inhomogeneous, intracellular force field. On this behalf, one might speculate that our AFM experiments, as they only accounted for the average swelling forces pointed upwards/towards the cantilever (chapter 3.1, figure 4e), usually underestimates the cell's original burst pressure. Hence, it could be interesting to see whether other, spatially resolving concepts are capable of ascertaining different, preferentially increased pressure values during NETosis. Here, optomechanical force sensors, such as presented by Gomez-Martinez et al. ${ }^{228}$ or Li et al. could provide a promising approach. Using such sensors in combination with nuclear live-cell imaging techniques, one could not only resolve local, intracellular stresses within the neutrophil but also directly relate them to the distribution of the associated chromatin network - an experiment of ultimate proof.

\subsection{A Theoretical Framework for Chemical Imaging Studies}

As discussed in chapter 2.2, one of the critical challenges of fluorescent nanosensor development is (and will be) the establishment of well-adjusted, characteristic response properties. Here, while functionalization strategies are vast and enable sensor modifications on multiple levels, the adaptation of the sensors response kinetics becomes one of the most essential aspects when targeting dynamic, biological events - even though such characteristics are hard to assess. Now, with the new strategy shown in chapter 3.2, computer-based screening methods could turn into a powerful tool to tackle this issue.

In this study, using a Monte-Carlo based simulation approach, we were able to emulate the interplay of analytes and sensor binding sites on a single molecule level and transformed this information into theoretical response images, usually obtained by microscope setups. In doing so, the novelty of this approach should be less seen in the simulation step per se, in fact, Boghossian et al. already demonstrated the power of theoretical sensor performance tests in early $2011^{229}$ - but rather in the integration of a multidimensional environment and the inclusion of spatiotemporal restrictions made by the theoretical "microscope". To explain this in more detail, one might visualize the benefits of such a more experiment-oriented concept with a simple case study. In common, one-dimensional simulation approaches it is normally assumed to work in a proximate perfect system. Single 
nanosensors are spatially resolvable, exposure times can be chosen as needed, and sample sizes do not matter (at least most of the time). ${ }^{230}$ Yet, in case of real experiments, these are crucial factors that need to be taken into consideration, particularly if the desired sensing event should result in spatiotemporal information of the observed system. Chapter 3.2, figure 4 is an excellent example of such occurrences. While, at high frame rates, the underlying sensor pattern can localize the release site of the associated dopamine release event (chapter 3.2, figure 4a), its spatial resolution capacity decreases in case of higher exposure times (chapter 3.2, figure 4c). Thus, it becomes evident that in real-life experiments the performance of a given sensor does not only depend on its interaction with the biological sample/analytes but also the settings of the corresponding imaging setup. For that reason, we created for the first time a theoretical chemical imaging framework that accounted for both of these factors. The initial Monte-Carlo simulation first evaluated the single sensor-analyte interaction (chapter 3.2, figure 2), while the consecutive introduction of diffraction patterns and frame integrations in the response image reproduced the behavior of an applied microscope. One might say, in a figurative matter of course, that we "constructed" a theoretical microscope setup of sorts.

Such a system has then one obvious significant advantage: it directly reflects the conditions of a real-time experiment. In turn this means, with the help of the generated theoretical response images, one is able to draw reliable conclusions on the outcome of an actual chemical imaging process, of course, insofar the properties of the theoretical system (e.g. analyte dynamics, spatial resolution, sensor concentration/geometry, etc.) resemble those of the experimental situation. This again has extensive application possibilities since the solution of the underlying equation system is not restricted to the properties of the applied sensors alone. Instead, it can be also used to assess the influence of any given variable. One might, for example, observe the impact of different sensor geometries (chapter 3.2, supp. fig. 4) or sizes (chapter 3.2, supp. fig. 5) on the outcome of the experiment in case the sensor kinetics are already known or stay constant. Likewise, exposure times (chapter 3.2, figure 4) or different "objectives" (magnifications, resolutions) can be tested to improve the experimental conditions. In other words, the prediction of the best sensor kinetics shown in chapter 3.2, figure 5 is only one of many applicabilities and, at the same time, needs to always be put in context with the chosen configurations of the system. On that account, the optimal sensor properties found in this example case $\left(\mathrm{k}_{\text {on }}=10^{6} \mathrm{M}^{-1} \mathrm{~s}^{-1}\right.$, $\mathrm{k}_{\text {off }}=10^{2} \mathrm{~s}^{-1}$, chapter 3.2, figure 5e) hold, but should also be constantly seen in conjunction with the theoretical microscope settings (e.g., exposure time of $t_{\text {int }}=10 \mathrm{~ms}$ or lens $\mathrm{f}$ number $\mathrm{f} / \#=1$ ) as well as resolution requirements (maximal spatial resolution in distances of $\mathrm{d}=500 \mathrm{~nm}$ ) we chose for the theoretical setup.

As a consequence, the next steps of this project should focus on an experimental proof of concept, predominantly to underline the applicability of the theoretical framework. In an 
ideal scenario, one could simply prepare a well-defined chemical imaging experiment, for example by exposing sensors to artificial, pre-defined analyte distributions and compare it to the results of the matched emulated system. In that way, one might prove if a simple Monte-Carlo simulation algorithm is sufficient enough to reconstruct the complex binding/unbinding interplay of the sensor-analyte construct or if more sophisticated mechanisms have to be applied. At the same time, a few in-depth modifications might help to enhance the overall adaptability of the system. On the one hand, one could try to implement "real" uptake events, that means a withdrawal of an associated analyte molecule from the system, whenever it is bound to one of the sensors binding sites. While this would barely affect the results of our sample cases, primarily due to the large number of excess analytes present in our studies, such interactions should represent a crucial factor when working close to single-molecule regimes. On the other hand, one might also try to account for the sensors absolute response intensities rather than working with relative values. Indeed, such an approach would entail large computational requirements and comprise complex effects, such as single-photon interactions or exciton-diffusion events, however, it would likewise result in outputs one step closer to reality.

\subsection{The Strength of Neutrophil-based Nanocarrier Systems}

Finally, the study shown in chapter $\mathbf{3 . 3}$ can be seen as a link between the two preceding topics; a merging work so to speak, in which we took advantage of the NETotic phase model introduced in chapter 3.1 and transformed neutrophils into a programmable carrier system for a nanoscale cargo, such as nanosensors described in chapter 3.2. In doing so, the overarching goal of this study comprised an ambitious aim: we wanted to create a biocompatible transport unit capable of active integration as well as cart and release of functional nanomaterial to a given destination. While such a system would be of great advantage for various biomedical applications, present nanocarriers usually lack at least one of these abilities. Transport via liposomes, for example, has already been proven to be a successful tool to deliver chemotherapeutic agents ${ }^{231}$. Yet, the inability of such compartments to actively seek and move to the site of action also results in low target ratios and unpredictable efficiencies. ${ }^{232}$ Vice versa, self-propelling micro-/nanomotorsystems, such as tubular microjets ${ }^{233}$, polymersomes ${ }^{234}$ or Janus particles ${ }^{235}$, provide an opportunity to trigger direct material shipment, but are most of the time also accompanied by unreliable uptake and/or release processes that hinder a final cargo submission. For that reason, it seemed appealing to test whether neutrophils, as a biological construct capable of incorporating materials (phago-/endocytosis), following mechanical/chemical cues (duro-/chemotaxis) and releasing intracellular content (NETosis), might define a more efficient way to deliver functional material. 
On this occasion, the idea of exploiting such cellular skill sets to transfer drug- or sensorrelated objects are actually not new. For instance, Chu et al. already used a mouse model to demonstrate that anti-CD11b (an integrin subunit of macrophage-1 antigen (Mac-1)) nanobodies can be attached on and carried by neutrophils in vivo which resulted in a new, potential strategy for cancer treatment. ${ }^{236}$ In the same manner, M1 macrophages were shown to be able to ingest poly(lactide-co-glycolide) nanoparticles, likewise in vivo, and carry them across the endothelial barrier of glioma-afflicted mice. ${ }^{237}$ Still, while both of these studies underline the tremendous potential of cell-mediated delivery systems, the general effectiveness of such an approach remains hitherto considerably vague. In particular, phagocytic cells were most often only demonstrated to function as a potential carrier by visualizing the uptake of a given cargo - however, strategies to purposefully release their content subsequently could not be determined so far. For this reason, the concept presented in chapter 3.3 can be considered as one of the first procedures in which all three mechanisms (uptake, transport and release) were combined and characterized to form a functional, cell-based transport system.

Therefore, special attention should be prevalently given to the two new perceptions that enabled this study in the first place: the first being that LPS-activated neutrophils are still able to migrate and chemotactically react within early P1 (chapter 3.3, figure 3-4); the second that incorporated SWCNT-based nanosensors remained functional across NETosis (chapter 3.3, figure 5). Here, while both findings constituted a necessity to build an operative, NETosis-based transport system, the former especially could also be of particular interest for prospective NET-research. As we were able to show, the general migration ability of activated neutrophils differs significantly depending on the amount and type of the added stimulants within the system (chapter 3.3, figure 3, suppl. fig. 2). While in our case an activation via LPS still resulted in the cells ability to move and thus formed the basis of our carrier model, the addition of larger amounts of PMA always implied a potent, yet abrupt, loss of all cellular mobility functions. On this behalf, it is fascinating to see that different stimuli (or their underlying signaling cascades) are capable of modifying the physical activity of neutrophils in varying ways - yet it similarly opens the question where these variations are descending from. Here, comparing the signaling pathway of both transducer types (see again chapter 2.1.4), one possibility could be the rather unique way PMA initiates the process. Unlike most physiological NET-inducers that start their signaling cascade by interacting with corresponding cell receptors, PMA is well known for bypassing these hurdles and directly activating PKCs. ${ }^{238}$ Thus, one might come to the inclination that such more "artificial" methods could limit the cells physiological functions whereas receptor-regulated activation enables a somewhat retention of its original abilities (at least as long as the degrading actomyosin cortex provides the possibility to do it). This would also explain why in one of our most 
recent studies LPS-activated neutrophils demonstrated a clear dependence on the elastic properties of their environment while PMA-pathways likewise seemed to be unaffected by any outer influence. ${ }^{239}$ For that reason, it would be interesting to test this hypothesis by, for example, continuing the migration experiments shown in chapter 3.3, figure 3-4 and utilizing additional inducer sources. In this way, one could further determine the effect of different NET-pathways on the physiological conditions of the cell and, at the same time, try to find out whether other stimuli provide even better pre-activation strategies to transform neutrophils into perfect carrier systems.

On the other hand, a word also ought to be devoted to the cargo we used to visualize the applicability of NETosis-based transporters: the SWCNT-sensors. While the observation that DNA-wrapped nanotubes can be incorporated by neutrophils alone represents a vital discovery (chapter 3.3, figure 2a), the circumstance that they remain operational and still are able to detect specific analytes throughout the NETotic process (chapter 3.3, figure 5) makes them a nearly perfect tool for phagocyte-mediated delivery systems. This is additionally underlined by the fact that so far strategies to incorporate functional nanomaterial into leukocytes have been rather limited or have required complex camouflage approaches to enforce active uptake events. ${ }^{232}$ It would be therefore an interesting premise to investigate whether these beneficial abilities only apply for specific SWCNT-modifications or if they hold for any kind of nanotube functionalization method. Especially in the latter case, follow-up projects including different, potential drug-related SWCNT customizations could enable interesting, new ambits for the here demonstrated carrier system. Zhang et al. reported for example a wide range of anti-cancer related nanotube functionalization strategies which would provide an excellent foundation for further investigations. ${ }^{240}$ Simultaneously, this would also enable new opportunities for in vivo applications. While our approach of direct cell-activation proved the general applicability of NETosis as a programmable release mechanism, it so far still requires an efficient strategy to target neutrophils within living organisms purposefully. On this regard, precise modification of SWCNTs could provide a helpful resource. By attaching NETosis-inducing compounds on associated SWCNTs, one could, for example, try to interconnect the internalization of the nanomaterial with the activation of the corresponding cell. With this, one could not only utilize the unique functionality of the nanometer-sized cargo but also ensure its timed, directed release without causing any prior (possibly adverse) pre-treatment of the entire system.

As a conclusion, the mechanism shown in chapter 3.3 should not only be seen as a novel approach to directly transport functional material within biological environments but also as a fascinating process in which two previously distinct research topics (NETosis and SWCNTs) are combined and can unfold their full potential. While the unique, multiphasic dynamics of NET-formation allow for programmable release mechanism that is 
unmatched by conventional carrier systems, the high modifiability and stability of SWCNTs as a cargo provide near endless amounts of possible applications. Thus, especially in these times of need for new, potential drug delivery mechanisms, NETosisbased nanocarrier systems could become of considerable importance. 



\section{Acknowledgment / Danksagung}

An letzter Stelle möchte ich einmal „Danke“ sagen an all die vielen Menschen, die mich über die letzten drei Jahre (und darüber hinaus) so tatkräftig unterstützt und mir eine wundervolle Doktorandenzeit beschert haben. Zuerst möchte ich mich hierbei besonders bei Sebastian Kruss und Luise Erpenbeck bedanken: Danke, nicht nur für eure langjährige Begleitung und die tollen Projekte, die ich mit und durch euch durchleben durfte, sondern auch für das immerzu familiäre Verhältnis, das durch eure ungezwungene und hilfsbereite Art zustande kam. Es war eine Ehre von „Anfang an“ dabei gewesen zu sein und das Wachsen der Gruppe und des Zusammenhaltes miterleben zu dürfen - Ich hoffe das bleibt euch noch lange Zeit erhalten.

Dank gebürt an dieser Stelle auch meinen TAC-Mitgliedern, Prof. Jörg Enderlein und Dr. Thomas Burg, die mich stets mit innovativen Ideen gefördert und es mir verziehen haben, wenn das eine oder andere Meeting mal ein wenig länger ausgefallen ist. Vielen Dank für Ihre Unterstützung! Zudem möchte ich an diesem Punkt auch Prof. Andreas Janshoff erwähnen, ohne dessen Hilfe einige Projekte erst gar nicht entstanden wären. Auch Ihnen vielen Dank für die langjährige Zuwendung.

Darüber hinaus gibt es noch eine lange Liste an weiteren Menschen, die ich gerne benennen möchte. Meine Eltern: Danke, dass ihr immer für mich da wart, an mich geglaubt und mir geholfen habt wo immer es nur ging - Ich könnte nicht stolzer sein, euer Sohn sein zu dürfen. Gleiches gilt für meine Schwester Mona: Ich hab“ dich lieb Schwesterherz.

Dann möchte ich mich noch bei (m)einem ganz besonderen Menschen bedanken: Uli, danke, dass du immer für mich da bist; für all die Zeiten, in denen du mich aufgemunterst und mich weitermachen lassen hast, wenn ich wieder kurz vor dem verzweifeln war und für all die Lasten, die du für mich aufgenommen hast. Ich liebe dich unheimlich sehr und freue mich über jeden Tag, den ich mit dir zusammen erleben darf.

Zudem gibt es da noch einen ganzen Haufen an Hallodries, die mir das Leben im Labor versüßt haben. Elsa Neubert: Ohne dich hätte ich es wohl nie so weit geschafft. Danke, dass du immer ein Ohr für mich hattest und für all die lustigen Momente - ich hätte mir keinen besseren Laborpartner wünschen können. Florian Mann: Irgendwann muss es mal jemand sagen - das mit Werder Bremen und der Champions League... naja..., aber vielleicht trinken wir da noch'n Bierchen drüber. Danke auch dir, ich freu' mich schon auf deine baldige Abholung! Robert Nissler: Endlich mal jemand mit gutem Musikgeschmack! Beim nächsten Rave bin ich dann dabei. Gabriele Selvaggio: Ditmaal up plattdüütsch dormit du ok maal nbehten Düütsch lehren deist. Büstn Guatsbleot, Gabi! Meshkat Dinarvand: For you in a more understandable language. Thanks for being there, I'm looking forward speaking with you in german in a few months ;-). Dann gibt es da noch unsere Laborkinder. Niklas Herrmann: Ohne dich würde ich Gänse immer noch unter anderen Gesichtspunkten sehen. Danke dir dafür. Alex Spreinat: Mach dir nichts draus, wir alle werden mal für älter geschätzt!

Zum Schluss möchte ich zudem noch meinen ehemaligen Studentinnen Annika Hagemann, Taija Fischer und Saba Telele für die tolle Zusammenarbeit danken. Gleiches gilt für alle Menschen des AK Janshoff, AK Steinem und AK Erpenbeck - obwohl immer mal wieder neue Gesichter dazukamen und alte gegangen sind, waren die Tage mit euch im Labor (vor allem aber in den Kaffeeräumen) immer was Besonderes.

Danke euch allen! 


\section{Bibliography}

1. Firestein, G. S., McInnes, I. B., O'Dell, J. R. \& Gabriel, S. E. eds. Kelley and Firestein's textbook of rheumatology (Elsevier, Philadelphia, PA, 2017).

2. Williams, W. J. Williams hematology (McGraw-Hill Education, New York, 2016).

3. Netea, M. G. et al. Trained immunity: A program of innate immune memory in health and disease. Science (New York, N.Y.) 352, aaf1098; 10.1126/science.aaf1098 (2016).

4. Boraschi, D. \& Italiani, P. Innate Immune Memory: Time for Adopting a Correct Terminology. Frontiers in immunology 9, 799; 10.3389/fimmu.2018.00799 (2018).

5. Nauseef, W. M. \& Borregaard, N. Neutrophils at work. Nature immunology 15, 602611; 10.1038/ni.2921 (2014).

6. Kay, A. B. Paul Ehrlich and the Early History of Granulocytes. Microbiology spectrum 4; 10.1128/microbiolspec.MCHD-0032-2016 (2016).

7. Borregaard, N., \& Cowland, J. B. Granules of the Human Neutrophilic Polymorphonuclear Leukocyte. Blood, 3503-3521 (1997).

8. Manley, H. R., Keightley, M. C. \& Lieschke, G. J. The Neutrophil Nucleus: An Important Influence on Neutrophil Migration and Function. Frontiers in immunology 9, 2867; 10.3389/fimmu.2018.02867 (2018).

9. Latt, S. A., Stetten, G., Juergens, L. A., Willard, H. F. \& Scher, C. D. Recent developments in the detection of deoxyribonucleic acid synthesis by 33258 Hoechst fluorescence. J Histochem Cytochem. 23, 493-505; 10.1177/23.7.1095650 (1975).

10. Aquiles Sanchez, J., Karni, R. J. \& Wangh, L. J. Fluorescent in situ hybridization (FISH) analysis of the relationship between chromosome location and nuclear morphology in human neutrophils. Chromosoma 106, 168-177 (1997).

11. Zucker-Franklin, D. Atlas of blood cells. Function and pathology. 2nd ed. (Edi. Ermes; Lea \& Febiger, Milano, Philadelphia, 1988).

12. Mollinedo, F. et al. Combinatorial SNARE complexes modulate the secretion of cytoplasmic granules in human neutrophils. Journal of immunology (Baltimore, Md. : 1950) 177, 2831-2841 (2006).

13. Ogawa, T., Koerten, H. K., Brederoo, P. \& Daems, W. T. A comparative study of primary and secondary granules in monocytopoiesis and myelopoiesis of mouse bone marrow. Cell and tissue research 228, 107-115 (1983). 
14. Rogovin, V. V., Fomina, V. A. \& Piruzyan, L. A. Electron cytochemistry of peroxidase activity in neutrophils of the bone marrow of the frog Rana temporaria. Biology bulletin of the Academy of Sciences of the USSR 5, 492-496 (1978).

15. O'Donnell, R. T. \& Andersen, B. R. Characterization of canine neutrophil granules. Infection and Immunity 38, 351-359 (1982).

16. Kjeldsen, L., Sengeløv, H., Lollike, K., Nielsen, M. H. \& Borregaard, N. Isolation and characterization of gelatinase granules from human neutrophils. Blood 83, 1640-1649 (1994).

17. Dewald, B., Bretz, U. \& Baggiolini, M. Release of gelatinase from a novel secretory compartment of human neutrophils. The Journal of clinical investigation 70, 518-525 (1982).

18. Borregaard, N., Sørensen, O. E. \& Theilgaard-Mönch, K. Neutrophil granules: a library of innate immunity proteins. Trends in immunology 28, 340-345; 10.1016/j.it.2007.06.002 (2007).

19. Hong, C.-W. Current Understanding in Neutrophil Differentiation and Heterogeneity. Immune Network 17, 298-306; 10.4110/in.2017.17.5.298 (2017).

20. Rosales, C. Neutrophil: A Cell with Many Roles in Inflammation or Several Cell Types? Frontiers in physiology 9, 113; 10.3389/fphys.2018.00113 (2018).

21. Görgens, A. et al. Revision of the human hematopoietic tree: granulocyte subtypes derive from distinct hematopoietic lineages. Cell reports 3, 1539-1552; 10.1016/j.celrep.2013.04.025 (2013).

22. Fiedler, K. \& Brunner, C. The role of transcription factors in the guidance of granulopoiesis. American Journal of Blood Research 2, 57-65 (2012).

23. Häger, M., Cowland, J. B. \& Borregaard, N. Neutrophil granules in health and disease. Journal of internal medicine 268, 25-34; 10.1111/j.1365-2796.2010.02237.x (2010).

24. Nourshargh, S. \& Alon, R. Leukocyte migration into inflamed tissues. Immunity 41, 694-707; 10.1016/j.immuni.2014.10.008 (2014).

25. Ekpenyong, A. E., Toepfner, N., Chilvers, E. R. \& Guck, J. Mechanotransduction in neutrophil activation and deactivation. Biochimica et biophysica acta 1853, 31053116; 10.1016/j.bbamcr.2015.07.015 (2015).

26. Dancey, J. T., Deubelbeiss, K. A., Harker, L. A. \& Finch, C. A. Neutrophil kinetics in man. The Journal of clinical investigation 58, 705-715; 10.1172/JCI108517 (1976). 
27. Köhler, A. et al. G-CSF-mediated thrombopoietin release triggers neutrophil motility and mobilization from bone marrow via induction of Cxcr2 ligands. Blood 117, 43494357; 10.1182/blood-2010-09-308387 (2011).

28. Eash, K. J., Greenbaum, A. M., Gopalan, P. K. \& Link, D. C. CXCR2 and CXCR4 antagonistically regulate neutrophil trafficking from murine bone marrow. The Journal of clinical investigation 120, 2423-2431; 10.1172//JCI41649 (2010).

29. Summers, C. et al. Neutrophil kinetics in health and disease. Trends in immunology 31, 318-324; 10.1016/j.it.2010.05.006 (2010).

30. Petit, I. et al. G-CSF induces stem cell mobilization by decreasing bone marrow SDF1 and up-regulating CXCR4. Nature immunology 3, 687-694; 10.1038/ni813 (2002).

31. Semerad, C. L. et al. G-CSF potently inhibits osteoblast activity and CXCL12 mRNA expression in the bone marrow. Blood 106, 3020-3027; 10.1182/blood-2004-01-0272 (2005).

32. Kim, H. K., La Luz Sierra, M. de, Williams, C. K., Gulino, A. V. \& Tosato, G. G-CSF down-regulation of CXCR4 expression identified as a mechanism for mobilization of myeloid cells. Blood 108, 812-820; 10.1182/blood-2005-10-4162 (2006).

33. Leiding, J. W. Neutrophil Evolution and Their Diseases in Humans. Frontiers in immunology 8; 10.3389/fimmu.2017.01009 (2017).

34. Oakes, P. W. et al. Neutrophil morphology and migration are affected by substrate elasticity. Blood 114, 1387-1395; 10.1182/blood-2008-11-191445 (2009).

35. Iba, T., Hashiguchi, N., Nagaoka, I., Tabe, Y. \& Murai, M. Neutrophil cell death in response to infection and its relation to coagulation. Journal of Intensive Care 1; 10.1186/2052-0492-1-13 (2013).

36. Bastian, O. W., Koenderman, L., Alblas, J., Leenen, L. P. H. \& Blokhuis, T. J. Neutrophils contribute to fracture healing by synthesizing fibronectin+ extracellular matrix rapidly after injury. Clinical immunology (Orlando, Fla.) 164, 78-84; 10.1016/j.clim.2016.02.001 (2016).

37. Cowburn, A. S., Condliffe, A. M., Farahi, N., Summers, C. \& Chilvers, E. R. Advances in Neutrophil Biology: Clinical Implications. Chest 134, 606-612; 10.1378/chest.08-0422 (2008).

38. Condliffe, A. M., Kitchen, E. \& Chilvers, E. R. Neutrophil priming: pathophysiological consequences and underlying mechanisms. Clinical science (London, England : 1979) 94, 461-471 (1998).

39. van Eeden, S. F., Klut, M. E., Walker, B. A. \& Hogg, J. C. The use of flow cytometry to measure neutrophil function. Journal of immunological methods 232, 23-43 (1999). 
40. Kitchen, E., Rossi, A. G., Condliffe, A. M., Haslett, C. \& Chilvers, E. R. Demonstration of reversible priming of human neutrophils using platelet-activating factor. Blood 88, 4330-4337 (1996).

41. Barnes, T. C., Anderson, M. E., Edwards, S. W. \& Moots, R. J. Neutrophil-derived reactive oxygen species in SSc. Rheumatology (Oxford, England) 51, 1166-1169; 10.1093/rheumatology/ker520 (2012).

42. Fernández-Segura, E., García, J. M., Santos, J. L. \& Campos, A. Shape, F-actin, and surface morphology changes during chemotactic peptide-induced polarity in human neutrophils. The Anatomical record 241, 519-528; 10.1002/ar.1092410410 (1995).

43. Swain, S. D., Rohn, T. T. \& Quinn, M. T. Neutrophil priming in host defense: role of oxidants as priming agents. Antioxidants \& redox signaling 4, 69-83; $10.1089 / 152308602753625870$ (2002).

44. MacKinnon, A. C. et al. Sphingosine Kinase: A Point of Convergence in the Action of Diverse Neutrophil Priming Agents. Journal of immunology (Baltimore, Md. : 1950) 169, 6394-6400; 10.4049/jimmunol.169.11.6394 (2002).

45. Furze, R. C. \& Rankin, S. M. The role of the bone marrow in neutrophil clearance under homeostatic conditions in the mouse. The FASEB Journal 22, 3111-3119; 10.1096/fj.08-109876 (2008).

46. Wright, H. L., Moots, R. J., Bucknall, R. C. \& Edwards, S. W. Neutrophil function in inflammation and inflammatory diseases. Rheumatology (Oxford, England) 49, 1618 1631; 10.1093/rheumatology/keq045 (2010).

47. Ley, K., Laudanna, C., Cybulsky, M. I. \& Nourshargh, S. Getting to the site of inflammation: the leukocyte adhesion cascade updated. Nature reviews. Immunology 7 , 678-689; 10.1038/nri2156 (2007).

48. Gotsch, U., Jäger, U., Dominis, M. \& Vestweber, D. Expression of P-selectin on endothelial cells is upregulated by LPS and TNF-alpha in vivo. Cell adhesion and communication 2, 7-14 (1994).

49. Phillipson, M. et al. Intraluminal crawling of neutrophils to emigration sites: a molecularly distinct process from adhesion in the recruitment cascade. The Journal of experimental medicine 203, 2569-2575; 10.1084/jem.20060925 (2006).

50. Smith, L. A., Aranda-Espinoza, H., Haun, J. B., Dembo, M. \& Hammer, D. A. Neutrophil traction stresses are concentrated in the uropod during migration. Biophysical journal 92, L58-60; 10.1529/biophysj.106.102822 (2007). 
51. Hind, L. E., Vincent, W. J. B. \& Huttenlocher, A. Leading from the Back: The Role of the Uropod in Neutrophil Polarization and Migration. Developmental cell 38, 161169; 10.1016/j.devcel.2016.06.031 (2016).

52. Sánchez-Madrid, F. \& Serrador, J. M. Bringing up the rear: defining the roles of the uropod. Nature Reviews Molecular Cell Biology 10, 353 EP -; 10.1038/nrm2680 (2009).

53. Friedl, P., Zänker, K. S. \& Bröcker, E. B. Cell migration strategies in 3-D extracellular matrix: differences in morphology, cell matrix interactions, and integrin function. Microscopy research and technique 43, 369-378; 10.1002/(SICI)10970029(19981201)43:5<369::AID-JEMT3>3.0.CO;2-6 (1998).

54. Friedl, P. \& Weigelin, B. Interstitial leukocyte migration and immune function. $\mathrm{Na}$ ture immunology 9, 960-969; 10.1038/ni.f.212 (2008).

55. Hoang, A. N. et al. Measuring neutrophil speed and directionality during chemotaxis, directly from a droplet of whole blood. Technology 1, 49-;

$10.1142 / \mathrm{S} 2339547813500040$ (2013).

56. Davidson, P. M., Denais, C., Bakshi, M. C. \& Lammerding, J. Nuclear deformability constitutes a rate-limiting step during cell migration in 3-D environments. Cellular and molecular bioengineering 7, 293-306; 10.1007/s12195-014-0342-y (2014).

57. Vestweber, D. VE-cadherin: the major endothelial adhesion molecule controlling cellular junctions and blood vessel formation. Arteriosclerosis, thrombosis, and vascular biology 28, 223-232; 10.1161/ATVBAHA.107.158014 (2008).

58. Filippi, M.-D. Mechanism of Diapedesis: Importance of the Transcellular Route. Advances in immunology 129, 25-53; 10.1016/bs.ai.2015.09.001 (2016).

59. Mócsai, A., Walzog, B. \& Lowell, C. A. Intracellular signalling during neutrophil recruitment. Cardiovascular research 107, 373-385; 10.1093/cvr/cvv159 (2015).

60. Gambardella, L. \& Vermeren, S. Molecular players in neutrophil chemotaxis--focus on PI3K and small GTPases. Journal of leukocyte biology 94, 603-612; 10.1189/jlb.1112564 (2013).

61. Woodfin, A. et al. JAM-A mediates neutrophil transmigration in a stimulus-specific manner in vivo: evidence for sequential roles for JAM-A and PECAM-1 in neutrophil transmigration. Blood 110, 1848-1856; 10.1182/blood-2006-09-047431 (2007).

62. Muller WA et al. PECAM-1 is required for transendothelial migration of leukocytes. The Journal of experimental medicine 178, 449-460 (1993). 
63. Feng, D., Nagy, J. A., Pyne, K., Dvorak, H. F. \& Dvorak, A. M. Neutrophils emigrate from venules by a transendothelial cell pathway in response to FMLP. The Journal of experimental medicine 187, 903-915 (1998).

64. Lossinsky, A. S. \& Shivers, R. R. Structural pathways for macromolecular and cellular transport across the blood-brain barrier during inflammatory conditions. Review. Histology and histopathology 19, 535-564; 10.14670/HH-19.535 (2004).

65. Wolburg, H., Wolburg-Buchholz, K. \& Engelhardt, B. Diapedesis of mononuclear cells across cerebral venules during experimental autoimmune encephalomyelitis leaves tight junctions intact. Acta neuropathologica 109, 181-190; 10.1007/s00401004-0928-x (2005).

66. Voisin, M.-B. \& Nourshargh, S. Neutrophil transmigration: emergence of an adhesive cascade within venular walls. Journal of innate immunity 5, 336-347; 10.1159/000346659 (2013).

67. Dangerfield, J., Larbi, K. Y., Huang, M.-T., Dewar, A. \& Nourshargh, S. PECAM-1 (CD31) Homophilic Interaction Up-Regulates $\alpha 6 \beta 1$ on Transmigrated Neutrophils In Vivo and Plays a Functional Role in the Ability of $\alpha 6$ Integrins to Mediate Leukocyte Migration through the Perivascular Basement Membrane. The Journal of experimental medicine 196, 1201-1212; 10.1084/jem.20020324 (2002).

68. Kienle, K. \& Lämmermann, T. Neutrophil swarming: an essential process of the neutrophil tissue response. Immunological reviews 273, 76-93; 10.1111/imr.12458 (2016).

69. Lämmermann, T. et al. Neutrophil swarms require LTB4 and integrins at sites of cell death in vivo. Nature 498, 371-375; 10.1038/nature12175 (2013).

70. Lee, W. L., Harrison, R. E. \& Grinstein, S. Phagocytosis by neutrophils. Microbes and infection 5, 1299-1306 (2003).

71. Richards, D. M. \& Endres, R. G. The mechanism of phagocytosis: two stages of engulfment. Biophysical journal 107, 1542-1553; 10.1016/j.bpj.2014.07.070 (2014).

72. Flannagan, R. S., Jaumouillé, V. \& Grinstein, S. The cell biology of phagocytosis. Annual review of pathology 7, 61-98; 10.1146/annurev-pathol-011811-132445 (2012).

73. Gordon, S. Phagocytosis: An Immunobiologic Process. Immunity 44, 463-475; 10.1016/j.immuni.2016.02.026 (2016).

74. Aderem, A. Phagocytosis and the inflammatory response. The Journal of infectious diseases 187 Suppl 2, S340-5; 10.1086/374747 (2003). 
75. Tang, D., Kang, R., Coyne, C. B., Zeh, H. J. \& Lotze, M. T. PAMPs and DAMPs: Signal 0s that Spur Autophagy and Immunity. Immunological reviews 249, 158-175; 10.1111/j.1600-065X.2012.01146.x (2012).

76. Futosi, K., Fodor, S. \& Mócsai, A. Neutrophil cell surface receptors and their intracellular signal transduction pathways $\underset{3}{ }$. International Immunopharmacology 17, 638650; 10.1016/j.intimp.2013.06.034 (2013).

77. Herant, M., Heinrich, V. \& Dembo, M. Mechanics of neutrophil phagocytosis: experiments and quantitative models. Journal of cell science 119, 1903-1913; 10.1242/jcs.02876 (2006).

78. Nordenfelt, P. \& Tapper, H. Phagosome dynamics during phagocytosis by neutrophils. Journal of leukocyte biology 90, 271-284; 10.1189/j1b.0810457 (2011).

79. Möhn, H., Le Cabec, V., Fischer, S. \& Maridonneau-Parini, I. The src-family proteintyrosine kinase p59hck is located on the secretory granules in human neutrophils and translocates towards the phagosome during cell activation. The Biochemical journal 309 ( Pt 2), 657-665 (1995).

80. Mollinedo, F., Martin-Martin, B., Calafat, J., Nabokina, S. M. \& Lazo, P. A. Role of Vesicle-Associated Membrane Protein-2, Through Q-Soluble N-EthylmaleimideSensitive Factor Attachment Protein Receptor/R-Soluble N-Ethylmaleimide-Sensitive Factor Attachment Protein Receptor Interaction, in the Exocytosis of Specific and Tertiary Granules of Human Neutrophils. Journal of immunology (Baltimore, Md. : 1950) 170, 1034-1042; 10.4049/jimmunol.170.2.1034 (2003).

81. Gustafson, H. H., Holt-Casper, D., Grainger, D. W. \& Ghandehari, H. Nanoparticle Uptake: The Phagocyte Problem. Nano today 10, 487-510; 10.1016/j.nantod.2015.06.006 (2015).

82. Bisso, P. W., Gaglione, S., Guimarães, P. P. G., Mitchell, M. J. \& Langer, R. Nanomaterial Interactions with Human Neutrophils. ACS Biomater. Sci. Eng. 4, 42554265; 10.1021/acsbiomaterials.8b01062 (2018).

83. Oh, Y. K. \& Swanson, J. A. Different fates of phagocytosed particles after delivery into macrophage lysosomes. The Journal of cell biology 132, 585-593 (1996).

84. Wu, L. et al. Tuning cell autophagy by diversifying carbon nanotube surface chemistry. ACS nano 8, 2087-2099; 10.1021/nn500376w (2014).

85. Fratti, R. A., Chua, J., Vergne, I. \& Deretic, V. Mycobacterium tuberculosis glycosylated phosphatidylinositol causes phagosome maturation arrest. Proceedings of the $\mathrm{Na}$ tional Academy of Sciences of the United States of America 100, 5437-5442; 10.1073/pnas.0737613100 (2003). 
86. Axelrod, S. et al. Delay of phagosome maturation by a mycobacterial lipid is reversed by nitric oxide. Cellular microbiology 10, 1530-1545; 10.1111/j.14625822.2008.01147.x (2008).

87. Paul, D. et al. Phagocytosis dynamics depends on target shape. Biophysical journal 105, 1143-1150; 10.1016/j.bpj.2013.07.036 (2013).

88. Gratton, S. E. A. et al. The effect of particle design on cellular internalization pathways. Proceedings of the National Academy of Sciences of the United States of America 105, 11613-11618; 10.1073/pnas.0801763105 (2008).

89. Lacy, P. Mechanisms of Degranulation in Neutrophils. Allergy, Asthma, and Clinical Immunology : Official Journal of the Canadian Society of Allergy and Clinical Immunology 2, 98-108; 10.1186/1710-1492-2-3-98 (2006).

90. Mayadas, T. N., Cullere, X. \& Lowell, C. A. The multifaceted functions of neutrophils. Annual review of pathology 9, 181-218; 10.1146/annurev-pathol-020712164023 (2014).

91. Faurschou, M. \& Borregaard, N. Neutrophil granules and secretory vesicles in inflammation. Microbes and infection 5, 1317-1327; 10.1016/j.micinf.2003.09.008 (2003).

92. Kurz, A. R. M. et al. MST1-dependent vesicle trafficking regulates neutrophil transmigration through the vascular basement membrane. The Journal of clinical investigation 126, 4125-4139; 10.1172/JCI87043 (2016).

93. Brinkmann, V. et al. Neutrophil extracellular traps kill bacteria. Science (New York, N.Y.) 303, 1532-1535; 10.1126/science. 1092385 (2004).

94. Martinod, K. \& Wagner, D. D. Thrombosis: tangled up in NETs. Blood 123, 27682776; 10.1182/blood-2013-10-463646 (2014).

95. Yu, Y. \& Su, K. Neutrophil Extracellular Traps and Systemic Lupus Erythematosus. Journal of clinical \& cellular immunology 4; 10.4172/2155-9899.1000139 (2013).

96. Papayannopoulos, V. Neutrophil extracellular traps in immunity and disease. Nature Reviews Immunology 18, 134 EP -; 10.1038/nri.2017.105 (2017).

97. Yang, H. et al. New Insights into Neutrophil Extracellular Traps: Mechanisms of Formation and Role in Inflammation. Frontiers in immunology 7; 10.3389/fimmu.2016.00302 (2016).

98. Martinelli, S. et al. Induction of genes mediating interferon-dependent extracellular trap formation during neutrophil differentiation. The Journal of biological chemistry 279, 44123-44132; 10.1074/jbc.M405883200 (2004). 
99. Kessenbrock, K. et al. Netting neutrophils in autoimmune small-vessel vasculitis. $\mathrm{Na}$ ture medicine 15, 623-625; 10.1038/nm.1959 (2009).

100. Garcia-Romo, G. S. et al. Netting neutrophils are major inducers of type I IFN production in pediatric systemic lupus erythematosus. Science translational medicine 3, 73ra20; 10.1126/scitranslmed.3001201 (2011).

101. Clark, S. R. et al. Platelet TLR4 activates neutrophil extracellular traps to ensnare bacteria in septic blood. Nature medicine 13, 463-469; 10.1038/nm1565 (2007).

102. Munks, M. W. et al. Aluminum adjuvants elicit fibrin-dependent extracellular traps in vivo. Blood 116, 5191-5199; 10.1182/blood-2010-03-275529 (2010).

103. Kaplan, M. J. \& Radic, M. Neutrophil extracellular traps (NETs): Double-edged swords of innate immunity1. Journal of immunology (Baltimore, Md. : 1950) 189, 2689-2695; 10.4049/jimmunol.1201719 (2012).

104. Yu, L., Quinn, M. T., Cross, A. R. \& Dinauer, M. C. Gp91phox is the heme binding subunit of the superoxide-generating NADPH oxidase. The Biochemical journal 95, 7993-7998; 10.1073/pnas.95.14.7993 (1998).

105. Khan, M. A. et al. Regulating NETosis: Increasing pH Promotes NADPH Oxidase-Dependent NETosis. Frontiers in medicine 5, 19; 10.3389/fmed.2018.00019 (2018).

106. Papayannopoulos, V., Metzler, K. D., Hakkim, A. \& Zychlinsky, A. Neutrophil elastase and myeloperoxidase regulate the formation of neutrophil extracellular traps. The Journal of cell biology 191, 677-691; 10.1083/jcb.201006052 (2010).

107. Hemmers, S., Teijaro, J. R., Arandjelovic, S. \& Mowen, K. A. PAD4-Mediated Neutrophil Extracellular Trap Formation Is Not Required for Immunity against Influenza Infection. PLoS ONE 6; 10.1371/journal.pone.0022043 (2011).

108. Li, P. et al. PAD4 is essential for antibacterial innate immunity mediated by neutrophil extracellular traps. The Journal of experimental medicine 207, 1853-1862; 10.1084/jem.20100239 (2010).

109. Tatsiy, O. \& McDonald, P. P. Physiological Stimuli Induce PAD4-Dependent, ROS-Independent NETosis, With Early and Late Events Controlled by Discrete Signaling Pathways. Frontiers in immunology 9, 2036; 10.3389/fimmu.2018.02036 (2018).

110. Douda, D. N., Khan, M. A., Grasemann, H. \& Palaniyar, N. SK3 channel and mitochondrial ROS mediate NADPH oxidase-independent NETosis induced by calcium influx. Proceedings of the National Academy of Sciences of the United States of America 112, 2817-2822; 10.1073/pnas.1414055112 (2015). 
111. Yousefi, S., Mihalache, C., Kozlowski, E., Schmid, I. \& Simon, H. U. Viable neutrophils release mitochondrial DNA to form neutrophil extracellular traps. Cell death and differentiation 16, 1438-1444; 10.1038/cdd.2009.96 (2009).

112. Yipp, B. G. \& Kubes, P. NETosis: how vital is it? Blood 122, 2784-2794; 10.1182/blood-2013-04-457671 (2013).

113. Kim, B. Y. S., Rutka, J. T. \& Chan, W. C. W. Nanomedicine. The New England journal of medicine 363, 2434-2443; 10.1056/NEJMra0912273 (2010).

114. Jain, A., Homayoun, A., Bannister, C. W. \& Yum, K. Single-walled carbon nanotubes as near-infrared optical biosensors for life sciences and biomedicine. Biotechnology journal 10, 447-459; 10.1002/biot.201400168 (2015).

115. Kruss, S. et al. Carbon nanotubes as optical biomedical sensors. Advanced drug delivery reviews 65, 1933-1950; 10.1016/j.addr.2013.07.015 (2013).

116. Liu, Z., Tabakman, S., Welsher, K. \& Dai, H. Carbon Nanotubes in Biology and Medicine: In vitro and in vivo Detection, Imaging and Drug Delivery. Nano research 2, 85-120; 10.1007/s12274-009-9009-8 (2009).

117. Shao, L., Gao, Y. \& Yan, F. Semiconductor Quantum Dots for Biomedicial Applications. Sensors (Basel, Switzerland) 11, 11736-11751; 10.3390/s111211736 (2011).

118. Ramgir, N. S., Yang, Y. \& Zacharias, M. Nanowire-based sensors. Small (Weinheim an der Bergstrasse, Germany) 6, 1705-1722; 10.1002/smll.201000972 (2010).

119. He, H. et al. Carbon nanotubes: applications in pharmacy and medicine. BioMed research international 2013, 578290; 10.1155/2013/578290 (2013).

120. Gao, C., Guo, Z., Liu, J.-H. \& Huang, X.-J. The new age of carbon nanotubes: an updated review of functionalized carbon nanotubes in electrochemical sensors. $\mathrm{Na}$ noscale 4, 1948-1963; 10.1039/c2nr11757f (2012).

121. Doria, G. et al. Noble Metal Nanoparticles for Biosensing Applications. Sensors (Basel, Switzerland) 12, 1657-1687; 10.3390/s120201657 (2012).

122. Barone, P. W., Baik, S., Heller, D. A. \& Strano, M. S. Near-infrared optical sensors based on single-walled carbon nanotubes. Nature Materials 4, 86 EP -; 10.1038/nmat1276 (2004).

123. Kruss, S. et al. High-resolution imaging of cellular dopamine efflux using a fluorescent nanosensor array. Proceedings of the National Academy of Sciences of the United States of America 114, 1789-1794; 10.1073/pnas.1613541114 (2017). 
124. Kim, J.-H. et al. Single-molecule detection of $\mathrm{H}_{2} \mathrm{O}_{2}$ mediating angiogenic redox signaling on fluorescent single-walled carbon nanotube array. ACS nano 5, 7848$7857 ; 10.1021 / \mathrm{nn} 201904 \mathrm{t}$ (2011).

125. Kam, N. W. S., O'Connell, M., Wisdom, J. A. \& Dai, H. Carbon nanotubes as multifunctional biological transporters and near-infrared agents for selective cancer cell destruction. Proceedings of the National Academy of Sciences of the United States of America 102, 11600-11605; 10.1073/pnas.0502680102 (2005).

126. Sinha, N. \& Yeow, J. T. W. Carbon nanotubes for biomedical applications. IEEE transactions on nanobioscience 4, 180-195 (2005).

127. Jin, H. et al. Detection of single-molecule $\mathrm{H} 2 \mathrm{O} 2$ signalling from epidermal growth factor receptor using fluorescent single-walled carbon nanotubes. Nature Nanotechnology 5, 302 EP -; 10.1038/nnano.2010.24 (2010).

128. Trojanowicz, M. Analytical applications of carbon nanotubes: a review. $\operatorname{Tr} A C$ Trends in Analytical Chemistry 25, 480-489; 10.1016/j.trac.2005.11.008 (2006).

129. Saito, R., Dresselhaus, G. \& Dresselhaus, M. S. Physical Properties of Carbon Nanotubes (PUBLISHED BY IMPERIAL COLLEGE PRESS AND DISTRIBUTED BY WORLD SCIENTIFIC PUBLISHING CO, 1998).

130. Dresselhaus, M. S., Dresselhaus, G. \& Avouris, P. Carbon Nanotubes. Synthesis, Structure, Properties, and Applications (Springer, Berlin, Heidelberg, 2001).

131. Ascheron, C. E., Duhm, A. H., Jorio, A., Dresselhaus, G. \& Dresselhaus, M. S. Carbon Nanotubes (Springer Berlin Heidelberg, Berlin, Heidelberg, 2008).

132. Odom, T. W., Huang, J.-L., Kim, P. \& Lieber, C. M. Atomic structure and electronic properties of single-walled carbon nanotubes. Nature 391, 62 EP -; 10.1038/34145 (1998).

133. Nitti, A., Pacini, A. \& Pasini, D. Chiral Nanotubes. Nanomaterials (Basel, Switzerland) 7; 10.3390/nano7070167 (2017).

134. Kataura, H. et al. Optical properties of single-wall carbon nanotubes. Synthetic Metals 103, 2555-2558; 10.1016/S0379-6779(98)00278-1 (1999).

135. Strano, M. S. Probing chiral selective reactions using a revised Kataura plot for the interpretation of single-walled carbon nanotube spectroscopy. Journal of the American Chemical Society 125, 16148-16153; 10.1021/ja036791x (2003).

136. van Hove, L. The Occurrence of Singularities in the Elastic Frequency Distribution of a Crystal. Phys. Rev. 89, 1189-1193; 10.1103/PhysRev.89.1189 (1953). 
137. Miyauchi, Y., Oba, M. \& Maruyama, S. Cross-polarized optical absorption of single-walled nanotubes by polarized photoluminescence excitation spectroscopy. Phys. Rev. B 74; 10.1103/PhysRevB.74.205440 (2006).

138. O'Connell, M. J. et al. Band gap fluorescence from individual single-walled carbon nanotubes. Science (New York, N.Y.) 297, 593-596; 10.1126/science.1072631 (2002).

139. Bachilo, S. M. et al. Structure-assigned optical spectra of single-walled carbon nanotubes. Science (New York, N.Y.) 298, 2361-2366; 10.1126/science.1078727 (2002).

140. Ghosh, S., Bachilo, S. M. \& Weisman, R. B. Advanced sorting of single-walled carbon nanotubes by nonlinear density-gradient ultracentrifugation. Nature Nanotechnology 5, 443-450; 10.1038/nnano.2010.68 (2010).

141. Miyauchi, Y. Photoluminescence studies on exciton photophysics in carbon nanotubes. J. Mater. Chem. C 1, 6499; 10.1039/c3tc00947e (2013).

142. Frenkel, J. On the Transformation of light into Heat in Solids. I. Phys. Rev. 37, 17-44; 10.1103/PhysRev.37.17 (1931).

143. Zhao, H., Mazumdar, S., Sheng, C.-X., Tong, M. \& Vardeny, Z. V. Photophysics of excitons in quasi-one-dimensional organic semiconductors: Single-walled carbon nanotubes and $\pi$-conjugated polymers. Phys. Rev. B 73;

10.1103/PhysRevB.73.075403 (2006).

144. Sekar, R. B. \& Periasamy, A. Fluorescence resonance energy transfer (FRET) microscopy imaging of live cell protein localizations. The Journal of cell biology 160, 629-633; 10.1083/jcb.200210140 (2003).

145. Kahmann, S. et al. Excited-State Interaction of Semiconducting Single-Walled Carbon Nanotubes with Their Wrapping Polymers. The Journal of Physical Chemistry Letters 8, 5666-5672; 10.1021/acs.jpclett.7b02553 (2017).

146. Kim, J.-H. et al. The rational design of nitric oxide selectivity in single-walled carbon nanotube near-infrared fluorescence sensors for biological detection. Nature Chemistry 1, 473 EP -; 10.1038/nchem.332 (2009).

147. He, X. et al. Carbon nanotubes as emerging quantum-light sources. Nature Materials 17, 663-670; 10.1038/s41563-018-0109-2 (2018).

148. Liu, Z. et al. In vivo biodistribution and highly efficient tumour targeting of carbon nanotubes in mice. Nature Nanotechnology 2, 47-52; 10.1038/nnano.2006.170 (2007). 
149. Liu, H., Nishide, D., Tanaka, T. \& Kataura, H. Large-scale single-chirality separation of single-wall carbon nanotubes by simple gel chromatography. Nature communications 2, 309; 10.1038/ncomms1313 (2011).

150. He, X., Wang, K. \& Cheng, Z. In vivo near-infrared fluorescence imaging of cancer with nanoparticle-based probes. Wiley interdisciplinary reviews. Nanomedicine and nanobiotechnology 2, 349-366; 10.1002/wnan.85 (2010).

151. Amiot, C. L., Xu, S., Liang, S., Pan, L. \& Zhao, J. X. Near-Infrared Fluorescent Materials for Sensing of Biological Targets. Sensors (Basel, Switzerland) 8, 3082$3105 ; 10.3390 / \mathrm{s} 8053082$ (2008).

152. Heller, D. A., Baik, S., Eurell, T. E. \& Strano, M. S. Single-Walled Carbon Nanotube Spectroscopy in Live Cells: Towards Long-Term Labels and Optical Sensors. Adv. Mater. 17, 2793-2799; 10.1002/adma.200500477 (2005).

153. Barone, P. W., Parker, R. S. \& Strano, M. S. In vivo fluorescence detection of glucose using a single-walled carbon nanotube optical sensor: design, fluorophore properties, advantages, and disadvantages. Analytical chemistry 77, 7556-7562; 10.1021/ac0511997 (2005).

154. Dresselhaus, M. S., Dresselhaus, G., Saito, R. \& Jorio, A. Raman spectroscopy of carbon nanotubes. Physics Reports 409, 47-99; 10.1016/j.physrep.2004.10.006 (2005).

155. Qi, J. S., Huang, J. Y., Feng, J., Shi, D. N. \& Li, J. The possibility of chemically inert, graphene-based all-carbon electronic devices with $0.8 \mathrm{eV}$ gap. ACS nano 5, 3475-3482; 10.1021/nn102322s (2011).

156. Carlson, L. J., Maccagnano, S. E., Zheng, M., Silcox, J. \& Krauss, T. D. Fluorescence efficiency of individual carbon nanotubes. Nano letters 7, 3698-3703; 10.1021/n1072014 (2007).

157. Yoon, H. et al. Periplasmic binding proteins as optical modulators of singlewalled carbon nanotube fluorescence: amplifying a nanoscale actuator. Angewandte Chemie (International ed. in English) 50, 1828-1831; 10.1002/anie.201006167 (2011).

158. Crochet, J., Clemens, M. \& Hertel, T. Quantum yield heterogeneities of aqueous single-wall carbon nanotube suspensions. Journal of the American Chemical Society 129, 8058-8059; 10.1021/ja071553d (2007).

159. Cha, T.-G. et al. Optical nanosensor architecture for cell-signaling molecules using DNA aptamer-coated carbon nanotubes. ACS nano 5, 4236-4244; 10.1021/nn201323h (2011). 
160. Ahn, J.-H. et al. Label-free, single protein detection on a near-infrared fluorescent single-walled carbon nanotube/protein microarray fabricated by cell-free synthesis. Nano letters 11, 2743-2752; 10.1021/n1201033d (2011).

161. Zaporotskova, I. V., Boroznina, N. P., Parkhomenko, Y. N. \& Kozhitov, L. V. Carbon nanotubes: Sensor properties. A review. Modern Electronic Materials 2, 95 $105 ; 10.1016 /$ j.moem.2017.02.002 (2016).

162. Bilalis, P., Katsigiannopoulos, D., Avgeropoulos, A. \& Sakellariou, G. Noncovalent functionalization of carbon nanotubes with polymers. RSC Adv 4, 29112934; 10.1039/C3RA44906H (2014).

163. Mallakpour, S. \& Soltanian, S. Surface functionalization of carbon nanotubes: fabrication and applications. $R S C A d v$. 6, 109916-109935; 10.1039/C6RA24522F (2016).

164. Zhang, J. et al. Molecular recognition using corona phase complexes made of synthetic polymers adsorbed on carbon nanotubes. Nature Nanotechnology 8, 959 EP -; 10.1038/nnano.2013.236 (2013).

165. Mann, F. A., Herrmann, N., Meyer, D. \& Kruss, S. Tuning Selectivity of Fluorescent Carbon Nanotube-Based Neurotransmitter Sensors. Sensors (Basel, Switzerland) 17; 10.3390/s17071521 (2017).

166. Kruss, S. et al. Neurotransmitter detection using corona phase molecular recognition on fluorescent single-walled carbon nanotube sensors. Journal of the American Chemical Society 136, 713-724; 10.1021/ja410433b (2014).

167. Karousis, N., Tagmatarchis, N. \& Tasis, D. Current progress on the chemical modification of carbon nanotubes. Chemical reviews 110, 5366-5397; 10.1021/cr100018g (2010).

168. Tasis, D., Tagmatarchis, N., Bianco, A. \& Prato, M. Chemistry of carbon nanotubes. Chemical reviews 106, 1105-1136; 10.1021/cr050569o (2006).

169. Singh, P. et al. Organic functionalisation and characterisation of single-walled carbon nanotubes. Chemical Society reviews 38, 2214-2230; 10.1039/b518111a (2009).

170. Liu, Z., Tabakman, S. M., Chen, Z. \& Dai, H. Preparation of carbon nanotube bioconjugates for biomedical applications. Nature protocols 4, 1372-1382; 10.1038/nprot.2009.146 (2009).

171. Zhao, Y.-L. \& Stoddart, J. F. Noncovalent functionalization of single-walled carbon nanotubes. Accounts of chemical research 42, 1161-1171; 10.1021/ar900056z (2009). 
172. Chen, Z. et al. Protein microarrays with carbon nanotubes as multicolor Raman labels. Nature biotechnology 26, 1285-1292; 10.1038/nbt.1501 (2008).

173. So, H.-M. et al. Single-walled carbon nanotube biosensors using aptamers as molecular recognition elements. Journal of the American Chemical Society 127, 1190611907; 10.1021/ja053094r (2005).

174. Strano, M. S. et al. Reversible, Band-Gap-Selective Protonation of Single-Walled Carbon Nanotubes in Solution. J. Phys. Chem. B 107, 6979-6985; 10.1021/jp027664a (2003).

175. Fujigaya, T. \& Nakashima, N. Non-covalent polymer wrapping of carbon nanotubes and the role of wrapped polymers as functional dispersants. Science and technology of advanced materials 16, 24802; 10.1088/1468-6996/16/2/024802 (2015).

176. Ben-Yoav, H., A. Schroeder, M. \& Noked, M. Chitosan bio-functionalization of carbon nanotube arrayed electrode. AML 8, 1166-1170; 10.5185/amlett.2017.1577 (2017).

177. Meyer, D., Hagemann, A. \& Kruss, S. Kinetic Requirements for Spatiotemporal Chemical Imaging with Fluorescent Nanosensors. ACS nano 11, 4017-4027; 10.1021/acsnano.7b00569 (2017).

178. Smart, S. K., Cassady, A. I., Lu, G. Q. \& Martin, D. J. The biocompatibility of carbon nanotubes. Carbon 44, 1034-1047; 10.1016/j.carbon.2005.10.011 (2006).

179. Wong, S. S., Harper, J. D., Lansbury, P. T. \& Lieber, C. M. Carbon Nanotube Tips: High-Resolution Probes for Imaging Biological Systems. Journal of the American Chemical Society 120, 603-604; 10.1021/ja9737735 (1998).

180. Liu, Z., Yang, K. \& Lee, S.-T. Single-walled carbon nanotubes in biomedical imaging. J. Mater. Chem. 21, 586-598; 10.1039/C0JM02020F (2011).

181. Nowak, D. et al. Nanoscale chemical imaging by photoinduced force microscopy. Science advances 2, e1501571; 10.1126/sciadv.1501571 (2016).

182. da Cunha, M. M. L. et al. Overview of chemical imaging methods to address biological questions. Micron (Oxford, England : 1993) 84, 23-36; 10.1016/j.micron.2016.02.005 (2016).

183. Lindon, J. C., Tranter, G. E. \& Koppenaal, D. W. eds. Encyclopedia of spectroscopy and spectrometry (Elsevier AP Academic Press, Amsterdam, Boston, Heidelberg, 2017).

184. Visualizing Chemistry: The Progress and Promise of Advanced Chemical Imaging (Washington (DC), 2006). 
185. Ogunleke, A. et al. 3D chemical imaging of the brain using quantitative IR spectro-microscopy. Chemical science 9, 189-198; 10.1039/c7sc03306k (2018).

186. Schroeder, V., Savagatrup, S., He, M., Lin, S. \& Swager, T. M. Carbon Nanotube Chemical Sensors. Chemical reviews 119, 599-663; 10.1021/acs.chemrev.8b00340 (2019).

187. Luo, Y., Kim, E. H., Flask, C. A. \& Clark, H. A. Nanosensors for the Chemical Imaging of Acetylcholine Using Magnetic Resonance Imaging. ACS nano; 10.1021/acsnano.8b01640 (2018).

188. Lemasson, F. A. et al. Selective dispersion of single-walled carbon nanotubes with specific chiral indices by poly(N-decyl-2,7-carbazole). Journal of the American Chemical Society 133, 652-655; 10.1021/ja105722u (2011).

189. Fong, D. \& Adronov, A. Recent developments in the selective dispersion of single-walled carbon nanotubes using conjugated polymers. Chemical science $\mathbf{8}, 7292$ 7305; 10.1039/c7sc02942j (2017).

190. Münich, P. W. et al. Tuning the Carbon Nanotube Selectivity: Optimizing Reduction Potentials and Distortion Angles in Perylenediimides. Journal of the American Chemical Society 140, 5427-5433; 10.1021/jacs.8b00452 (2018).

191. Choi, Y. et al. in Carbon Nanotubes, Graphene, and Associated Devices VI, edited by D. Pribat, Y. H. Lee, M. Razeghi \& S. Baik (SPIE2013), 88140Q.

192. Juan, A. de, López-Moreno, A., Calbo, J., Ortí, E. \& Pérez, E. M. Determination of association constants towards carbon nanotubes. Chemical science 6, 7008-7014; $10.1039 / \mathrm{c} 5 \mathrm{sc} 02916 \mathrm{c}(2015)$.

193. Oh, H., Sim, J. \& Ju, S.-Y. Binding affinities and thermodynamics of noncovalent functionalization of carbon nanotubes with surfactants. Langmuir : the ACS journal of surfaces and colloids 29, 11154-11162; 10.1021/la4022933 (2013).

194. Upadhyay, S. K. Chemical Kinetics and Reaction Dynamics. 1st ed. (SpringerVerlag, s.1., 2006).

195. Smith, I. W. M. Reaction Dynamics (Springer, Boston, MA, 1980).

196. Levitus, M. Chemical Kinetics at the Single-Molecule Level. J. Chem. Educ. 88, 162-166; 10.1021/ed100371m (2011).

197. Gillespie, D. T. Stochastic simulation of chemical kinetics. Annual review of physical chemistry 58, 35-55; 10.1146/annurev.physchem.58.032806.104637 (2007).

198. Lecca, P. Stochastic chemical kinetics: A review of the modelling and simulation approaches. Biophysical Reviews 5, 323-345; 10.1007/s12551-013-0122-2 (2013). 
199. Macnamara, S., Bersani, A. M., Burrage, K. \& Sidje, R. B. Stochastic chemical kinetics and the total quasi-steady-state assumption: application to the stochastic simulation algorithm and chemical master equation. The Journal of chemical physics 129, 95105; 10.1063/1.2971036 (2008).

200. Jansen, A. P. J. An introduction to kinetic Monte Carlo simulations of surface reactions (Springer, Heidelberg, 2012).

201. Konno, H. \& Tamura, Y. Approximate time-dependent solution of a master equation with full linear birth-death rates. J. Phys. Commun. 2, 25033; 10.1088/23996528/aaae13 (2018).

202. Horowitz, J. M. Diffusion approximations to the chemical master equation only have a consistent stochastic thermodynamics at chemical equilibrium. The Journal of chemical physics 143, 44111; 10.1063/1.4927395 (2015).

203. Voter, A. F., pp. 1-23.

204. Révész, P., Birnbaum, Z. W. \& Lukacs, E. The Laws of Large Numbers (Elsevier Science, Burlington, 2014).

205. Gillespie, D. T. Exact stochastic simulation of coupled chemical reactions. $J$. Phys. Chem. 81, 2340-2361; 10.1021/j100540a008 (1977).

206. Erban, R., Chapman, J. \& Maini, P. A practical guide to stochastic simulations of reaction-diffusion processes (2007).

207. Robert Nißler, Florian Alexander Mann, Parth Chaturvedi, Jan Horlebein, Daniel Meyer, Lela Vukovic, and Sebastian Kruss. Quantification of the Number of Adsorbed DNA Molecules on Single-Walled Carbon Nanotubes. The Journal of Physical Chemistry (2019).

208. Kenny, E. F. et al. Diverse stimuli engage different neutrophil extracellular trap pathways. eLife 6; 10.7554/eLife.24437 (2017).

209. Metzler, K. D., Goosmann, C., Lubojemska, A., Zychlinsky, A. \& Papayannopoulos, V. A myeloperoxidase-containing complex regulates neutrophil elastase release and actin dynamics during NETosis. Cell reports 8, 883-896; 10.1016/j.celrep.2014.06.044 (2014).

210. Broers, J. L. V. et al. Decreased mechanical stiffness in LMNA-/- cells is caused by defective nucleo-cytoskeletal integrity: implications for the development of laminopathies. Human molecular genetics 13, 2567-2580; 10.1093/hmg/ddh295 (2004).

211. Salvermoser, M., Begandt, D., Alon, R. \& Walzog, B. Nuclear Deformation During Neutrophil Migration at Sites of Inflammation. Frontiers in immunology 9, 2680; 10.3389/fimmu.2018.02680 (2018). 
212. Jacobson, E. C. et al. Migration through a small pore disrupts inactive chromatin organization in neutrophil-like cells. BMC biology 16, 142; 10.1186/s12915-0180608-2 (2018).

213. Sollberger, G. et al. Gasdermin D plays a vital role in the generation of neutrophil extracellular traps. Science immunology 3; 10.1126/sciimmunol.aar6689 (2018).

214. Olins, D. E. \& Olins, A. L. Granulocyte heterochromatin: defining the epigenome. BMC cell biology 6, 39; 10.1186/1471-2121-6-39 (2005).

215. Olins, A. L. et al. The human granulocyte nucleus: unusual nuclear envelope and heterochromatin composition. European journal of cell biology 87, 279-290; 10.1016/j.ejcb.2008.02.007 (2008).

216. Jiang, D., Saffarzadeh, M. \& Scharffetter-Kochanek, K. In vitro Demonstration and Quantification of Neutrophil Extracellular Trap Formation. BIO-PROTOCOL 7; 10.21769/BioProtoc.2386 (2017).

217. Bont, C. M. de, Koopman, W. J. H., Boelens, W. C. \& Pruijn, G. J. M. Stimulusdependent chromatin dynamics, citrullination, calcium signalling and ROS production during NET formation. Biochimica et biophysica acta. Molecular cell research $\mathbf{1 8 6 5}$, 1621-1629; 10.1016/j.bbamcr.2018.08.014 (2018).

218. Veith, K. et al. Lipid-like Peptides can Stabilize Integral Membrane Proteins for Biophysical and Structural Studies. Chembiochem 18, 1735-1742; 10.1002/cbic.201700235 (2017).

219. Stewart, M. P. et al. Hydrostatic pressure and the actomyosin cortex drive mitotic cell rounding. Nature 469, 226-230; 10.1038/nature09642 (2011).

220. Charras, G. T., Coughlin, M., Mitchison, T. J. \& Mahadevan, L. Life and times of a cellular bleb. Biophysical journal 94, 1836-1853; 10.1529/biophysj.107.113605 (2008).

221. Keren, K., Yam, P. T., Kinkhabwala, A., Mogilner, A. \& Theriot, J. A. Intracellular fluid flow in rapidly moving cells. Nature cell biology 11, 1219-1224; 10.1038/ncb1965 (2009).

222. Rodríguez-Espinosa, O., Rojas-Espinosa, O., Moreno-Altamirano, M. M. B., López-Villegas, E. O. \& Sánchez-García, F. J. Metabolic requirements for neutrophil extracellular traps formation. Immunology 145, 213-224; 10.1111/imm.12437 (2015).

223. Kelly, S. M. \& Macklem, P. T. Direct measurement of intracellular pressure. The American journal of physiology 260, C652-7; 10.1152/ajpcell.1991.260.3.C652 (1991). 
224. Koslov, M. M. \& Markin, V. S. A theory of osmotic lysis of lipid vesicles. Journal of theoretical biology 109, 17-39 (1984).

225. VanHook, A. M. Stimulating NETosis instead of mitosis. Sci. Signal. 10, eaar6334; 10.1126/scisignal.aar6334 (2017).

226. Fischer-Friedrich, E., Hyman, A. A., Jülicher, F., Müller, D. J. \& Helenius, J. Quantification of surface tension and internal pressure generated by single mitotic cells. Scientific Reports 4, 6213 EP -; 10.1038/srep06213 (2014).

227. Yu, X., Tan, J. \& Diamond, S. L. Hemodynamic force triggers rapid NETosis within sterile thrombotic occlusions. Journal of thrombosis and haemostasis : JTH 16, 316-329; 10.1111/jth.13907 (2018).

228. Gómez-Martínez, R. et al. Silicon chips detect intracellular pressure changes in living cells. Nature Nanotechnology 8, 517-521; 10.1038/nnano.2013.118 (2013).

229. Boghossian, A. A. et al. The chemical dynamics of nanosensors capable of singlemolecule detection. The Journal of chemical physics 135, 84124; 10.1063/1.3606496 (2011).

230. Beyene, A. G., McFarlane, I. R., Pinals, R. L. \& Landry, M. P. Stochastic Simulation of Dopamine Neuromodulation for Implementation of Fluorescent Neurochemical Probes in the Striatal Extracellular Space. ACS chemical neuroscience 8, 22752289; 10.1021/acschemneuro.7b00193 (2017).

231. Kumar, P., Gulbake, A. \& Jain, S. K. Liposomes a vesicular nanocarrier: potential advancements in cancer chemotherapy. Critical reviews in therapeutic drug carrier systems 29, 355-419 (2012).

232. Shao, J. et al. Chemotaxis-Guided Hybrid Neutrophil Micromotors for Targeted Drug Transport. Angew. Chem. 129, 13115-13119; 10.1002/ange.201706570 (2017).

233. Restrepo-Pérez, L., Soler, L., Martínez-Cisneros, C. S., Sánchez, S. \& Schmidt, O. G. Trapping self-propelled micromotors with microfabricated chevron and heartshaped chips $\uparrow \dagger$ Electronic supplementary information (ESI) available: Supporting videos (S1; S2 and S3). See DOI: 10.1039/c3lc51419fClick here for additional data file.Click here for additional data file.Click here for additional data file.Click here for additional data file. Lab on a Chip 14, 1515-1518; 10.1039/c31c51419f (2014).

234. Peng, F., Tu, Y., van Hest, J. C. M. \& Wilson, D. A. Self-Guided Supramolecular Cargo-Loaded Nanomotors with Chemotactic Behavior towards Cells. Angewandte Chemie (International ed. in English) 54, 11662-11665; 10.1002/anie.201504186 (2015). 
235. Walther, A. \& Müller, A. H. E. Janus particles: synthesis, self-assembly, physical properties, and applications. Chemical reviews 113, 5194-5261; 10.1021/cr300089t (2013).

236. Chu, D., Dong, X., Shi, X., Zhang, C. \& Wang, Z. Neutrophil-Based Drug Delivery Systems. Advanced materials (Deerfield Beach, Fla.) 30, e1706245; 10.1002/adma.201706245 (2018).

237. Pang, L., Zhu, Y., Qin, J., Zhao, W. \& Wang, J. Primary M1 macrophages as multifunctional carrier combined with PLGA nanoparticle delivering anticancer drug for efficient glioma therapy. Drug delivery 25, 1922-1931;

10.1080/10717544.2018.1502839 (2018).

238. Alemán, O. R., Mora, N., Cortes-Vieyra, R., Uribe-Querol, E. \& Rosales, C. Differential Use of Human Neutrophil Fc $\gamma$ Receptors for Inducing Neutrophil Extracellular Trap Formation. Journal of Immunology Research 2016; 10.1155/2016/2908034 (2016).

239. Erpenbeck, L. et al. Effect of Adhesion and Substrate Elasticity on Neutrophil Extracellular Trap Formation: Supplementary Information (2019).

240. Zhang, W., Zhang, Z. \& Zhang, Y. The application of carbon nanotubes in target drug delivery systems for cancer therapies. Nanoscale research letters $\mathbf{6}, 555$; 10.1186/1556-276X-6-555 (2011). 